Universitat Politècnica de Catalunya

Consejo Superior de Investigaciones Científicas

\title{
Testing the Initial-Final Mass Relationship of White DWarfs \\ by
}

Silvia Catalán Ruiz

A THESIS SUBMITTED FOR THE DEGREE OF

Doctor of Phylosophy

AdVISORS:

JORDI ISERN VILABOY,

Enrique García-Berro Montilla 

A mis padres y hermana 



\section{Agraïments}

En primer lloc vull agrair als meus directors de tesi, Jordi Isern i Enrique GarcíaBerro, que em donessin l'oportunitat de realitzar el doctorat a l'IEEC (al CSIC i a la UPC), així com que em proporcionessin tot el suport necessari. Crec que he après moltes coses importants en aquests últims anys, no només en la part científica, i això en part és una conseqüència d'haver treballat amb ells. Els vull donar les gràcies també pel suport i la confiança dipositada en mi, especialment durant aquest últim any de feina.

Durant tota aquesta època he tingut la sort de tenir uns companys fantàstics que m'han fet molt més fàcil la feina. Primer vaig conèixer l'Alina, la meva companya de "fatigas" i després el Jose, el Pep i el Miquel. Més tard s'hi van afegir l'Ignasi, la Violeta, l'Anna C., l'Anna A., el Carlo, el Moi, el Nil, l'Anaïs... Tots els moments que hem compartit dins i fora de l'IEEC han estat molt importants per a mi i m'emporto un gran record de tota aquesta època, primer al Nexus, després a la UAB i finalment a l'ETSE. Ara que tinc la oportunitat, els hi vull agrair als meus companys, o més ben dit, amics, tots els ànims i suport que m'han donat. També els hi vull donar les gràcies al personal d'administració i a la resta de companys que he tingut durant aquests anys.

En especial, l'Ignasi es mereix un gran gràcies... Primer de tot, per venir a l'IEEC en un moment en que la meva feina necessitava d'un observador amb experiència. Per tenir la generositat de compartir els seus coneixements amb mi durant aquests anys, per acompanyar-me en les observacions, per tenir sempre tanta confiança en mi, molta més que jo mateixa i per ajudar-me sempre que se li presentés la oportunitat. Els seus ànims i el seu suport han estat essencials per a que arribés aquest moment i per tant, només em queda donar-li el meu agraïment més sincer.

También le debo un agradecimiento especial a Carlos Allende Prieto, que sin ni siquiera conocerme en persona aceptó acompañarme a observar al McDonald Observatory durante 10 días. Su ayuda y dedicación en la distancia, especialmente en el análisis de las compañeras de las enanas blancas, han sido también vitales para el desarrollo de esta tesis.

Durante mi doctorado he tenido la oportunidad de disfrutar de estancias en otros centros. Le quiero dar las gracias a Inma Domínguez, Carlos Abia y Olga Zamora de la Universidad de Granada por la atención que me dispensaron cuando estuve allí. También quisiera dar las gracias a Roberto Silvotti y Juan Alcalá por la 
ayuda y el buen trato que me dieron durante el tiempo que pasé en el Osservatorio Astronomico di Capodimonte. Gracias también al resto de personas que trabajan en el Osservatorio y que hicieron mi estancia muy agradable.

A mi profesor Manolo Segura le quiero agredecer que en su día me hiciera creer en mis posibilidades y despertara mi afán por intentar superarme poco a poco. Muchas gracias también por la "contingencia" y por la confianza dipositada en mí.

En un momento para mí tan importante como éste no me puedo olvidar de todos mis amigos fuera del IEEC. Por fortuna son unos cuantos, así que me perdonen los que no salen aquí. Realmente, esta historia empezó hace 10 años cuando me fui a Tenerife a estudiar Física (bueno, Astrofísica). Allí tuve la suerte de encontrar a grandes amigos: Laia, Juan Pedro, Idafe, Ale, Gabri, Iballa, Ana y Enol. El pensar que aunque lejos están ahí, siempre me ha dado fuerzas para afrontar los retos que se me han ido presentando. Les quiero agradecer la confianza y cariño que me han dado todos estos años. En especial, a Ana y Enol les quiero agradecer en primer lugar que vinieran a Barcelona, ya que juntos hemos conseguido que la conexión con el resto fuera más fuerte. Les doy también las gracias por todo el apoyo y ánimos que me han dado durante este tiempo.

Tampoco me puedo olvidar de mis amigos de Mollet. Muchas gracias a todos por los momentos de distracción y risas. A Noemí S., le doy las gracias por la primera llamada, por su apoyo y por escucharme siempre que lo he necesitado. A Noemí, "mandrágora", moltes gràcies per estar sempre al meu costat, per donar-me ànims i per no deixar que la problemàtica espai-temps afectés mínimament la nostra amistat. A l'Anna també li vull donar les gràcies pel seu suport i per totes les estones compartides que han fet més fàcil el camí final.

Mis mayores agradecimientos van para toda mi familia (tíos, primos, abuelos...) por todo el apoyo que me han proporcionado desde siempre. Por creer que lo que hacía era importante, aunque raro. Por hacer piña cuando ha hecho falta y sobretodo, por seguir celebrando comidas juntos aunque ya casi no quepamos, y por muchos años espero! En especial, al Joan li vull donar les gràcies per escoltar-me, per l'interès que ha demostrat sempre en alló que feia i pel seu suport. A mi hermana Esther le doy las gracias por no dejar nunca que me sienta sola, por su gran apoyo y por creer siempre en mis capacidades. Por todo el esfuerzo realizado durante el tiempo que hemos estado separadas y por quererme tanto. Finalmente a mis padres les quiero dar las gracias por hacerme sentir siempre que mis ilusiones eran también las suyas. Por ser un excelente ejemplo y demostrarme que con trabajo y esfuerzo uno puede conseguir aquello que se proponga. Muchas gracias por la paciencia que han tenido conmigo estos años y por su apoyo y dedicación constante.

Des d'un punt de vista material, l'el-laboració d'aquesta tesi ha estat subvencionada pel Ministeri d'Educació i Ciència (MEC) mitjançant una beca de formació de professorat universitari (FPU). També ha estat parcialment finançada pels projectes del MEC AYA05-08013-C03-01/02, per la Unió Europea (FEDER), per l'IEEC i per l'AGAUR. 


\section{Contents}

Motivation and outline of the thesis iii

1 Introduction 1

1.1 Evolution of low- and intermediate-mass stars . . . . . . . . . 2

1.2 Central stars of planetary nebulae and white dwarfs . . . . . . . 3

1.3 Unsolved problems . . . . . . . . . . . . . . . . . 4

1.4 How to measure the initial-final mass relationship? . . . . . . . . . 8

1.4.1 Open clusters and wide binaries . . . . . . . . . . . 8

1.4.2 The white dwarf luminosity function and the white dwarf mass

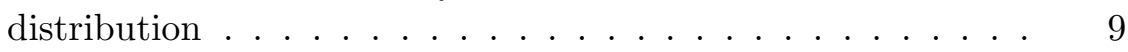

1.4.3 Overview of the methods used in this work . . . . . . . 9

$\begin{array}{lll}2 & \text { White dwarfs in common proper motion pairs } & 11\end{array}$

2.1 Introduction . . . . . . . . . . . . . . . . . 11

2.2 Observations and data reduction $\ldots \ldots \ldots \ldots$

2.3 White dwarf analysis . . . . . . . . . . . . . . . 16

2.3 .1 Classification . . . . . . . . . . . . . . . 16

2.3.2 Atmospheric parameters . . . . . . . . . . . . 16

2.3.3 Masses and cooling times . . . . . . . . . . . . . 21

2.4 Low-mass members analysis . . . . . . . . . . . . . . . . 23

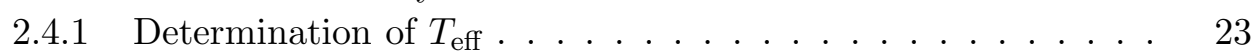

2.4 .2 Determination of $[\mathrm{Fe} / \mathrm{H}] \ldots \ldots \ldots \ldots$

2.4 .3 Age determination . . . . . . . . . . . . . . . 31

2.5 Masses of the progenitors . . . . . . . . . . . . . 33

$\begin{array}{lll}3 & \text { The peculiar case of WD0433+270 } & 37\end{array}$

3.1 Introduction . . . . . . . . . . . . . . . . . 37

3.2 Properties of the pair . . . . . . . . . . . . . . . 37

3.3 Membership to the Hyades . . . . . . . . . . . . . . . . . . 39

3.4 The nature of WD0433+270 . . . . . . . . . . . . . 41

3.5 Discussion . . . . . . . . . . . . . . . . . . 45 
4 Revision of the current initial-final mass relationship 49

4.1 Introduction . . . . . . . . . . . . . . . . . 49

4.2 Available data . . . . . . . . . . . . . . . . . 50

4.2 .1 NGC $2099(\mathrm{M} 37) \ldots \ldots \ldots \ldots \ldots$

$4.2 .2 \quad \mathrm{NGC} 2168(\mathrm{M} 35) \ldots \ldots \ldots \ldots$

4.2 .3 Other available data . . . . . . . . . . . . . . 63

4.3 Discussion . . . . . . . . . . . . . . . . . . 67

4.4 Final remarks . . . . . . . . . . . . . . . . . . 75

$\begin{array}{lll}5 & \text { The white dwarf luminosity function } & \mathbf{7 7}\end{array}$

5.1 Introduction . . . . . . . . . . . . . . . . . 77

5.2 The influence of the progenitors . . . . . . . . . . 78

5.3 The luminosity function of massive white dwarfs . . . . . . . . . 84

5.4 Discussion . . . . . . . . . . . . . . . . . . . . . . 89

6 An optical survey: the Alhambra Survey 91

6.1 Description of the project . . . . . . . . . . . . . . . 92

6.2 Photometric identification of white dwarfs . . . . . . . . . . 95

6.2.1 Color-color diagrams . . . . . . . . . . . . . . . . . . 101

6.2.2 Color-magnitude diagrams . . . . . . . . . . . . . . . . 103

6.2.3 Cool white dwarfs . . . . . . . . . . . . . . . . . 104

6.3 Number of white dwarfs in the Alhambra survey . . . . . . . . . . 107

6.4 Current status of the project . . . . . . . . . . . . . . 109

$\begin{array}{lll}7 & \text { Summary and conclusions } & 111\end{array}$

$\begin{array}{lr}\text { A White dwarf cooling sequences } & 117\end{array}$

A.1 Basic theory of cooling . . . . . . . . . . . . . . . 117

A.2 Carbon-oxygen white dwarfs . . . . . . . . . . . . 117

A.3 Oxygen-neon white dwarfs . . . . . . . . . . . . . . . . 122

$\begin{array}{lr}\text { B Stellar evolutionary tracks } & 127\end{array}$

B.1 The treatment of convection . . . . . . . . . . . . . . . 128

B.2 Mass-loss processes . . . . . . . . . . . . . . . . . . . . 129 


\section{Motivation and outline of the thesis}

White dwarfs are the final remnants of low- and intermediate-mass stars. About $95 \%$ of main-sequence stars will end their evolutionary pathways as white dwarfs and, hence, the study of the white dwarf population provides details about the late stages of the life of the vast majority of stars. Since white dwarfs are long-lived objects, they also constitute useful objects to study the structure and evolution of our Galaxy (Liebert et al. 2005a; Isern et al. 2001). For instance, the initial-final mass relationship, which connects the final mass of a white dwarf with the initial mass of its main-sequence progenitor, is of paramount importance for different aspects in modern astrophysics. This function is used for determining the ages of globular clusters and their distances, for studying the chemical evolution of galaxies, and also to understand the properties of the Galactic population of white dwarfs. Despite its relevance, this relationship is still relatively poorly constrained.

The initial-final mass relationship can be determined using two different approaches. The first approach involves an indirect measurement. This is done by studying the influence of the initial-final mass relationship in the two fundamental tools typically used to statistically analyze the properties of the white dwarf population: the white dwarf luminosity function and the white dwarf mass distribution. It is important to realize that the former has also been consistently used to obtain estimates of the age of the Galactic disk (Dantona \& Mazzitelli 1978; Winget et al. 1987; García-Berro et al. 1988; Richer et al. 2000) and the past history of the galactic star formation rate (Noh \& Scalo 1990; Díaz-Pinto et al. 1994). The latter yields important information about the late stages of stellar evolution since it reveals the amount of mass lost during stellar evolution from an initial stellar mass distribution (Liebert et al. 2005a). The second approach to improve the initial-final mass relationship consists in determining the masses of white dwarfs for which other constraints can be observationally derived. Since this method involves the use of theoretical stellar evolutionary tracks the resulting initial-final mass relationship is, in fact, semi-empirical.

From an observational perspective, the statistical significance of the current semiempirical initial-final mass relationship can be improved by performing spectroscopic 
observations of white dwarfs for which some important parameters, such as their total age or metallicity, are available. Most of the works carried out up to now have focused in studying white dwarfs in open clusters (Ferrario et al. 2005, Dobbie et al. 2006), whose ages can be determined from model fits to the turn-off location in a color-magnitude diagram. One of the aims of this work is to increase the number of observational data points by studying white dwarfs in a different context, namely in common proper motion pairs, for which the total age of the system can be derived. Note that the total age of a white dwarf can be expressed as the sum of its cooling time and the main-sequence lifetime of its progenitor. The latter depends on the metallicity of the progenitor of the white dwarf, but it cannot be determined from observations of single white dwarfs. This is because white dwarfs have such strong surface gravities that gravitational settling operates very efficiently in their atmospheres, and any information about their progenitors (and, in particular, about the metallicity) is lost in the very early evolutionary stages of the cooling track. Moreover, the evolution during the asymptotic giant branch (AGB) phase of their progenitors is essential in determining the size and composition of the atmospheres of the resulting white dwarfs, since the burning processes that take place in the hydrogen and helium shells determine their respective thicknesses and their detailed chemical compositions, which are crucial ingredients for determining the evolutionary cooling times. In chapter 1 we describe the evolution of the low- and intermediatemass stars, which are the progenitors of white dwarfs, placing special emphasis in describing the AGB phase. In this chapter we also introduce the parameters that may affect the initial-final mass relationship and list the main problems regarding this relevant relationship that are pending to be solved.

In chapter 2 we present a promising approach to circumvent some of the problems previously mentioned. In particular, in this chapter we describe in detail the results of an observational campaign devoted to measure the spectroscopic properties of a handful of white dwarfs in non-interacting binaries and, more precisely, in common proper motion pairs. This allows a direct test of the initial-final mass relationship. For these systems it is sound to assume that both members of the common proper motion pair were born simultaneously and with the same chemical composition (Wegner 1973; Oswalt et al. 1988). Since the components are well separated (100 to $1000 \mathrm{AU}$ ), mass exchange between them is unlikely and it can be considered that they have evolved as isolated stars. Thus, important information about the white dwarf, such as its total age and the metallicity of the progenitor, can be inferred from the study of the companion. We have studied several common proper motion pairs comprised of a white dwarf and a FGK star. We have performed spectroscopic observations of both members of each pair, and a careful analysis of the observational data has allowed us to derive the initial and final masses of the white dwarfs. It is worth mentioning that this is the first time that common proper motion pairs are used with the purpose of improving the initial-final mass relationship.

Chapter 3 is devoted to study a particular white dwarf for which very surprising 
results have been obtained during the analysis of the spectroscopic data collected in the course of this work and, hence, posing a certain challenge to the method described in chapter 2. The results arising from the analysis of the spectroscopic data indicate that either this white dwarf has an iron core, which contradicts our current understanding of the standard evolutionary paths leading to the formation of a white dwarf, or it belongs to the Hyades stream. Both possibilities are puzzling and deserve further study, and this is the reason why we devote a separate chapter to describe the results obtained so far.

In chapter 4 we re-evaluate the cluster data currently used to define the semiempirical initial-final mass relationship. We have used different white dwarf cooling sequences and different stellar evolutionary tracks in order to evaluate their influence on the final and initial masses derived. Using the results based on common proper motion pairs obtained in chapter 2 and the data obtained in open cluster we make a revision of the semi-empirical initial-final mass relationship. We also intend to give some clues on the dependence of this function on different parameters, especially with metallicity.

Although the main aim of this thesis is to perform a direct test of the initialfinal mass relationship, we decided to study also this relationship from a theoretical point of view. In particular, we have evaluated its influence on the white dwarf luminosity function. This is done in chapter 5 , where we compute the white dwarf luminosity function using different stellar evolutionary tracks and several initial-final mass relationships and we compare our results with the current observational data. We have also used the recent data obtained by Liebert et al. (2005a) to compare the theoretical white dwarf luminosity function of massive white dwarfs with the available observational results and evaluate its dependence on the several determinations of the initial-final mass relationships available in the literature, including the semi-empirical relationship derived in the previous chapter.

Chapter 6 is devoted to the Alhambra Survey. As it will be shown below, the need of increasing the amount of accurate and reliable observational data is clear. In this regard it is important to note that new deep surveys, of which the Alhambra Survey is a good example, will offer the possibility of substantially increasing the statistical significance of both the white dwarf luminosity function and of the mass distribution of white dwarfs. These surveys will also detect thousands of new white dwarfs, some of them belonging to common proper motion pairs, which could be eventually used to extend our analysis. Thus, we have performed an exhaustive study to optimize the identification procedure of the white dwarf candidates which will be eventually detected by the Alhambra survey. Finally, chapter 7 is devoted to summarize our main results and findings and to elaborate our conclusions. 



\section{Chapter 1}

\section{Introduction}

The initial-final mass relationship connects the mass of a white dwarf (final mass) with the mass of its progenitor in the main sequence (initial mass). This function is a key ingredient to study several important aspects of modern Astrophysics. As a matter of fact, it is required as an input for determining the ages of globular clusters and their distances, for studying the chemical evolution of galaxies, to determine the rate of core collapse supernovae, to understand the star formation and feedback processes in galaxies, and for studying the properties of the galactic populations of field and cluster white dwarfs. Despite its critical importance, this function is at present relatively poorly constrained, both from the theoretical and the observational points of view. The first attempt to empirically determine the initial-final mass relationship was undertaken by Weidemann (1977), who also has provided a recent revision (Weidemann 2000). Weidemann (1977) established the basis of the most common method used to derive the initial and final masses of white dwarfs. This method typically consists in performing spectroscopic observations of white dwarfs in open clusters and deriving their initial and final masses using cooling sequences and stellar evolutionary tracks. Since this procedure combines theoretical estimates with observational data, the resulting relation is commonly called the semi-empirical initial-final mass relationship. Weidemann (1977) also performed a comparison of measurements of the initial and final masses of white dwarfs in open clusters to theoretical predictions using different approaches to express the loss-mass processes. Since then, many improvements have been achieved, from both the theoretical and observational points of view. At the present time, owing to the increasing number of open cluster white dwarf studies and to the development of modern instrumentation, the semi-empirical initial-final mass relationship contains data from $\sim 50$ white dwarfs. Despite this fact, there are still some missing points in the puzzle. For instance, it is still not clear the dependence, if any, of the initial-final mass relationship with the mass and metallicity of the progenitor, angular momentum, magnetic field. . . 
In order to identify the main parameters that may have an influence in the initialfinal mass relationship of white dwarfs, a correct understanding of the evolution of their progenitors is of critical importance. In this chapter we give a brief description of the evolution of low- and intermediate-mass stars and we describe the pending problems and difficulties which hamper the determination of a reliable initial-final mass relationship.

\subsection{Evolution of low- and intermediate-mass stars}

White dwarfs are the evolutionary end-product of low- and intermediate-mass stars, which are those stars with masses $M \lesssim 12 M_{\odot}$. Low- and intermediate-mass stars spend most part of their lifes burning hydrogen into helium in the main-sequence. The duration of this phase decreases as mass increases. As a result, these stars share similar evolutionary characteristics, although some differences do exist depending on the mass of the star. This, in turn, translates into different observational and structural characteristics, as well as different evolutionary tracks in the HertzsprungRussell diagram, which represents the luminosity of a star as a function of its effective temperature.

When a star exhausts its initial hydrogen supply in the core, the main sequence phase arrives to its end and the star begins to contract until the temperature and density are high enough to ignite hydrogen in a shell around the hydrogen-exhausted core. This, in turn, causes the star to expand and climb up the Red Giant Branch (RGB). Eventually, a point is reached on the RGB in which the hydrogen-exhausted core reaches the helium ignition temperature and helium burning starts. After this, the star burns helium in a rather stable way in the so-called helium main sequence. However, the duration of this phase is much shorter than that of the central hydrogen burning phase. When helium is exhausted in the core, the star begins to burn helium in a shell around the carbon core - very much in the same manner as the hydrogen burning shell is established - and the star moves in the Hertzsprung-Russell diagram towards the Asymptotic Giant Branch (AGB). In this phase, the helium-burning shell converts helium into carbon and oxygen, increasing the mass of the carbon-oxygen $(\mathrm{C} / \mathrm{O})$ core. At the same time, the core contracts slowly and, as a consequence, its central density and temperature increase. When the helium-burning shell gets very close to the hydrogen-rich envelope, the hydrogen shell is re-ignited and increases the size of the helium buffer, with the hydrogen-burning shell supplying most of the luminosity. At a certain point, the mass of the helium buffer reaches a critical mass and becomes unstable, causing a helium shell flash. When such an event takes place, the helium shell suddenly releases a large amount of energy in a very short timescale, causing the expansion and cooling of the hydrogen burning shell. The net result of the expansion of the hydrogen shell is that nuclear reactions are effectively extinguished. As a consequence, the inner edge of the convective envelope moves 
inwards and dredges-up helium-rich material to the surface. At the same time, since hydrogen burning is effectively switched-off, the hydrogen-rich radiative region just below the convective envelope is compressed and its temperature increases. This, in turn, leads to a re-ignition of the hydrogen shell, which again is responsible of almost all of the luminosity of the star, and the whole process repeats again. This cycle is known as a thermal pulse. It is worth mentioning that when the progenitor star is massive enough $\left(\sim 8.0 M_{\odot}\right)$ and its core exceeds $\sim 1.05 M_{\odot}$, carbon is ignited off-center in semidegenerate conditions before reaching the thermally pulsing phase at the AGB. In this case, a series of carbon-burning off-center shell flashes take place in the inner core and the resulting degenerate remnant is made of oxygen and neon $(\mathrm{O} / \mathrm{Ne})$, instead of $\mathrm{C} / \mathrm{O}$. Nevertheless stars with $\mathrm{O} / \mathrm{Ne}$ cores also experience a thermally pulsing phase when the carbon burning phase is over.

Thermal pulses are thought to drive efficient mass loss and, thus, to remove the envelope of the star leaving as a remnant a white dwarf. In fact, regardless of the core composition, during the thermally pulsing phase the envelope of the star is rather unstable and the mass-loss rate can be very high. Unfortunately, our understanding of the mechanisms that cause mass loss is still poor. Consequently, the number of thermal pulses necessary to remove the whole envelope is not yet known, although very probably varies from star to star depending on, at least, the metallicity and the mass of the star. It is though generally accepted that the mass-loss mechanism is physically linked to the hydrogen and helium shell flashes, since during this phase the mass of the envelope of the star continuously decreases (while the radius and luminosity increase) and this implies that the surface material is less tightly bound. Although the exact cause of mass-loss is still not known, its influence on the evolution of AGB stars is significant. What is clear is that during this final stage, the core never becomes large and hot enough to ignite nuclear burning. Instead, the densities in the core become very large and the pressure of degenerate electrons is dominant. On the contrary, the final fate of stars with larger masses $\left(M \gtrsim 12 M_{\odot}\right)$ is completely different. These stars go through all the burning stages and produce a Type II supernova.

\subsection{Central stars of planetary nebulae and white dwarfs}

During the final stages of the mass-loss phase, the star leaves the AGB, since its effective temperature increases at nearly constant luminosity. The photosphere becomes very bright because of the absorption of photons by the ejected material and the star is now seen as a planetary nebula. Then, the ejected hydrogen- and helium-burning shells are extinguished and the luminosity of the star drops rapidly, revealing a white dwarf at the center of the planetary nebula. White dwarfs are located on the lower left part of the Hertzsprung-Russell diagram, since they usually have high effective temperatures but low luminosities, due to their small radius. 
As previously mentioned, according to theoretical calculations not all the white dwarfs have a core made of carbon and oxygen, although this is the most common composition. For instance, white dwarfs with masses less than $0.4 M_{\odot}$ are made of helium. The existence of this low-mass population was revealed by Liebert et al. (2005a) as a result of the analysis of the mass distribution of DA white dwarfs in the Palomar-Green Survey. Helium-core white dwarfs have been interpreted as a direct consequence of the evolution of close binary systems, although Kilic et al. (2007) in a recent work claim that helium white dwarfs may exist in the Galaxy also as a product of single evolution. On the other hand, white dwarfs with masses larger than $\sim 1.05 M_{\odot}$ are made of oxygen and neon (García-Berro \& Iben 1994). These massive white dwarfs are the product of the single evolution of stars with initial masses between 8 and $\sim 12 M_{\odot}$. The range of masses between the populations of $\mathrm{He}$ and $\mathrm{O} / \mathrm{Ne}$ white dwarfs is covered by $\mathrm{C} / \mathrm{O}$ core white dwarfs. The exact composition of the $\mathrm{C} / \mathrm{O}$ core critically depends on the evolution during the previous AGB phase. In a typical white dwarf of $0.57 M_{\odot}$ (Kepler et al. 2007), oxygen would represent $62 \%$ of the total mass, and its concentration in the central layers of the white dwarf could be as high as $85 \%$ (Salaris et al. 2000). Independently of the exact ratio between carbon and oxygen, the core is surrounded by a thin layer of pure helium with a mass ranging from $10^{-2}$ to $10^{-4} M_{\odot}$, and a even thinner layer of hydrogen of $10^{-4}$ to $10^{-15} M_{\odot}$, which is not always present. Although these outer layers are very thin, they are opaque to radiation and regulate the energy outflow from the star. They consequently play an essential role in the cooling of a white dwarf.

From a observational perspective, we can only obtain information about the outer regions. Using the spectral features that appear in white dwarf atmospheres, they can be classified as DA stars, if they present hydrogen absorption lines, or as non-DA stars, if they do not show hydrogen spectral features. About $85 \%$ of white dwarfs are of the DA type, and, in fact, these are the most well-understood white dwarfs at the moment. The atmospheres of DA white dwarfs can be well reproduced, since they have fully radiative, pure-hydrogen photospheres whose opacity sources can be calculated to high precision. Thus, the fit of the observed Balmer lines to model atmospheres can provide the atmospheric parameters (effective temperature and surface gravity) of these objects with excellent accuracy . For all these reasons, in this work we will only consider white dwarfs of this type.

\subsection{Unsolved problems}

After outlining the main physical processes that take place in low- and intermediatemass stars since its birth until they become white dwarfs, it is clear that the initialfinal mass relationship may depend on a huge number of parameters. The observational determination of the initial-final mass relationship is also a difficult task and has not been undertaken until relatively recent dates. Thus, the theoretical models 
cannot be properly compared with observations. Additionally, we will show in the next paragraphs that this relationship depends on different parameters apart from the initial mass of the star in the main sequence:

- Metallicity: it is clear that the chemical composition of a star should have a strong effect on its evolution. The smaller the metallicity of the star, the shorter the time that the star spends in the main sequence. Small values of $Z$ induce a reduction of the opacity without a reduction of the radius. Therefore, in order to maintain the structure, the temperature and the luminosity have to increase with the subsequent reduction in the lifetime of the star. At the same time, the helium core that is left is larger and the white dwarf that finally results is more massive than those obtained from progenitors with the same mass but larger metallicities (Schwarzschild 1958). However, the dependence of the initialfinal mass relationship of white dwarfs with metallicity is far from being well established. A star suffers many different processes during its pre-white dwarf life which may also depend on its chemical composition. Thus, it is reasonable to suspect that the initial-final mass relationship is metallicity dependent. In fact, all the theoretical initial-final mass relationships computed until now (e.g. Domínguez et al. 1999; Marigo 2001) predict that a more metallic progenitor will end up as a less massive white dwarf. From an observational perspective this dependence has not been clearly confirmed. In a recent work, Kalirai et al. (2005) have claimed to have found some indication that the initial-final mass relationship may depend on metallicity. On the other hand, the thorough study of Williams (2007) did not corroborate such dependence. In fact, this author found that for an initial mass of $3 M_{\odot}$, metallicity should affect the final mass of a white dwarf only in $0.05 M_{\odot}$.

- Rotation: Due to the conservation of angular momentum white dwarfs should be very fast rotators if a significant fraction of the angular momentum of the progenitor stars were to be preserved. However, for most white dwarfs, only upper limits on the rotational velocities in the range between 20 and $40 \mathrm{~km} \mathrm{~s}^{-1}$ are available (Karl et al. 2005). Thus, the general conclusion is that white dwarfs are slow rotators. This result is supported by astereoseismological investigations of pulsating white dwarfs. These small rotational velocities indicate that the angular momentum of the stellar cores is efficiently transported to the stellar surface and carried away by the mass lost during the course of stellar evolution. In any case, the rotational velocity of the progenitor star should have had an impact in the final structure of the white dwarf.

The contraction of the $\mathrm{C} / \mathrm{O}$ core at the onset of the AGB phase of low- and intermediate-mass stars may induce a significant increase in angular momentum, even for initially slowly rotating stars. Although a self-consistent treatment of stellar rotation is not yet available, Domínguez et al. (1996) used a 
simplified approach to show that if rotation was included in the calculation of stellar models, the final mass obtained was larger. In particular, they obtained that an initial star of $6.5 M_{\odot}$ with a large rotational velocity develops a white dwarf with a mass between 1.1 and $1.4 M_{\odot}$, depending on the initial conditions. The physical conditions near the center of the rotating models of Domínguez et al. (1996) resemble those of non-rotating models for smaller masses, typically $\sim 1.5 M_{\odot}$ less massive. Thus, it is reasonable to suspect that the initial-final mass relationship should have a dependence on rotation, since it affects the final structure of white dwarfs.

- Magnetism: according to Wickramasinghe \& Ferrario (2005), magnetic white dwarfs with fields in excess of $\sim 10^{6} \mathrm{G}$ constitute about $10 \%$ of all white dwarfs and show a mass distribution with a mean mass of $\sim 0.93 M_{\odot}$, which is much larger than the typical value of $\sim 0.56 M_{\odot}$ (Bergeron et al. 1992), which corresponds to non-magnetic white dwarfs. However, this could be just a bias caused by the influence of the magnetic field on the shape of the Balmer lines that mimics the presence of a larger gravitational field. Wickramasinghe \& Ferrario (2005) developed different magnetic white dwarf models and reached the conclusion that the initial-final mass relationship is essentially unaffected by the presence of a magnetic field, and that the reason why magnetic white dwarfs are typically more massive than non-magnetic ones is because their progenitors are also more massive. The magnetic field of magnetic white dwarfs could be then a fossil remnant from the main-sequence phase consistent with the approximate magnetic flux conservation.

Although the most favoured scenario to explain the effect of the magnetic field in the final structure of a white dwarf is that explained above, other possibilities cannot be ruled out. For instance, the presence of a magnetic field in the progenitor star could affect its evolution developing a more massive white dwarf, and thus, the initial-final mass relationship should be dependent on the strength of the magnetic field.

The effect of magnetic fields in stellar evolution can be evaluated by studying the magnetic white dwarf population from an observational perspective . Magnetic white dwarfs can be identified either by performing spectropolarimetric observations (Aznar Cuadrado et al. 2004) or by looking for Zeeman splitting in the Balmer lines (Koester et al. 1998). From these two techniques, spectropolarimetry is the most promising, since Zeeman splitting becomes undetectable for weak fields.

- Mass loss and expulsion mechanism: as previously pointed out, during the ascent along the AGB, an envelope removal is believed to occur after a certain number of thermal pulses. However, the moment at which this occurs and the physical mechanisms responsible for such an occurrence are still a matter of 
debate. During the thermal pulses, which are induced by the instabilities of the hydrogen- and helium-burning shells, the mass of the $\mathrm{C} / \mathrm{O}$ core increases as a result of the accretion of carbon freshly synthesized by the helium flashes. In the absence of mass loss, the mass of the $\mathrm{C} / \mathrm{O}$ core would eventually grow enough to approach the Chandrasekhar mass and finally the star would undergo a violent, disruptive carbon ignition. However, the observed luminosity of AGB stars in our Galaxy, as well as the observed mass-loss rates in stars at the AGB tip, seem to indicate that the occurrence of too many pulses is prevented by the removal of the envelope. Moreover, it is also thought that mass-loss processes are sensitive to metallicity, increasing their efficiency for smaller values of metallicity, which would affect the initial-final mass relationship. Due to all these uncertainties, mass-loss processes are still not well reproduced from a theoretical perspective. Mass loss, together with the mechanism of expulsion represent one of the most important problems pending to solve since they have a great impact on the final structure of white dwarfs.

- Convection: the elucidation of the real behaviour of convection is one of the most crucial points in stellar evolution. The mixing of material in the core of a given star significantly alters its lifetime, since it defines the value of $M_{\text {crit }}$, which is the maximum initial mass of a star able to develop a degenerate $\mathrm{C} / \mathrm{O}$ core, and thus, able to reach the AGB phase. The instability against turbulent convection is classically handled by means of a thermodynamical criterion (the Schwarzschild criterion for a chemically homogeneous fluid). This criterion is based on the evaluation of the expected gradient of temperature produced by the radiative transport of energy. When the required gradient is too high, convection accounts for the energy transport instead of radiative flux. It exists though, the possibility that the convective motion is not drastically inhibited in a stable region located just outside the convective core. In that case, a mechanical overshoot might induce a mixing of material in a radiatively stable region, contributing also to energy transport. There are several models that include this phenomenon, called overshooting. These approaches for convection imply different consequences on the development of the core of the star. Nowadays, the most commonly used model is the mixing length theory. Although basically a phenomenological theory, containing arbitrary constants, it is generally quite successful in predicting results of observations. Unfortunately, this is not a universal theory and needs a calibration. Consequently, the exact value of the mass transition between $\mathrm{He}$ and $\mathrm{C} / \mathrm{O}$ white dwarfs and between the latter and $\mathrm{O} / \mathrm{Ne}$ white dwarfs, and the theoretical predictions relating the main-sequence mass and the white dwarf mass are always model dependent.

Models assuming overshooting have smaller values of $M_{\text {crit }}$ in comparison with models considering the standard Schwarschild criterion to define the limits of the convective boundaries. The main effects of including overshooting in stel- 
lar models can be summarized as the following: during the core H-burning phase, the star has a larger He core, a larger luminosity, and a longer lifetime. During the following core He-burning phase, the luminosity is larger and the lifetime is shorter than in the absence of overshooting. Thus, models that consider overshooting predict larger core masses than those without overshooting. Moreover, the extra mixing due to overshooting could increase the metallicity of the outer layers and, consequently change the mass-loss rates, which is also dependeent on this parameter.

\subsection{How to measure the initial-final mass relationship?}

As already mentioned, there are two ways to test the initial-final mass relationship: either directly, by performing spectroscopic observations of white dwarfs for which external constraints are available, or indirectly, by studying its influence on the white dwarf luminosity function and on the mass distribution of white dwarfs. We briefly describe both of them.

\subsubsection{Open clusters and wide binaries}

Generally speaking, observations of isolated white dwarfs cannot reveal the properties of their progenitors during the main sequence phase, since they have such strong surface gravities that gravitational settling operates very efficiently in their atmospheres. Consequently, any information about the metallicity of their progenitors, which has a key role in calculating the overall age of single white dwarfs is lost (progenitor lifetime plus cooling time) in the very early evolutionary stages of the cooling phase. Most of the efforts up to now have focused in the observation of white dwarfs in open clusters, since for these stellar systems there are independent measurements which allow to infer the age of the cluster and the original metallicity of the progenitors of white dwarfs (Ferrario et al. 2005; Dobbie et al. 2006). Open clusters have made possible the derivation of a semi-empirical initial-final mass relationship containing about 50 white dwarfs. At the beginning of this work, the relation roughly covered the initial mass range between $\sim 2.5$ and $\sim 7 M_{\odot}$. This is because open clusters are relatively young and the white dwarf progenitors in these groups are generally massive. Consequently, they have short main-sequence lifetimes. The unavoidable limitation of these stellar systems led us to search for white dwarfs in a different context that may be also valid for the purpose of improving the initial-final mass relationship. This is the case of white dwarfs in wide binaries or common proper motion pairs in which one of the members is a low-mass star, still in the first phases of evolution, whereas the companion is a white dwarf. These systems are adequate for our purpose since it can be assumed that the members of a common proper motion pair were born simultaneously and with the same chemical 
composition (Wegner 1973; Oswalt et al. 1988) and thus, observations of the lowmass companion allow to restrict the space of parameters for the white dwarf. In particular, from a detailed analysis of the spectra of both members of the pair and the use of stellar evolutionary tracks the initial and final mass of the white dwarf can be derived.

\subsubsection{The white dwarf luminosity function and the white dwarf mass distribution}

From a theoretical point of view, there are two fundamental tools for studying the properties of the white dwarf population: the white dwarf luminosity function and the white dwarf mass distribution. Both of them can be computed from very basic theoretical grounds and have reliable observational determinations to which they can be compared to. Thus, indirect tests of the initial-final mass relationship can be done by comparing the theoretical predictions of different initial-final mass relationships with the available observational data. We will only use one of these distributions, the white dwarf luminosity function. The white dwarf luminosity function is defined as the number of white dwarfs per unit volume and per bolometric magnitude. This function depends on different input parameters: the star formation rate, the initial mass function, the main-sequence lifetime of the progenitors of white dwarfs, the initial-final mass relationship... With respect to the mass distribution of white dwarfs it can be said that understanding its precise shape offers a sorely needed insight about the total amount of mass lost during the course of the last phases of stellar evolution. Consequently, the theoretical modelling of this function and the comparison with the observational data allows to constrain the initial-final mass relationship. Nevertheless, we will not model this distribution.

\subsubsection{Overview of the methods used in this work}

The main aim of this work is to obtain a better observational determination of the initial-final mass relationship. For such a purpose we will primarily use white dwarfs in common proper pairs. Then, we will add these new data to the available results in the literature to obtain a new semi-empirical initial-final mass relationship. From a theoretical perspective, we will carry out a series of theoretical calculations of the white dwarf luminosity function. We will also evaluate the dependence of this function with different star formation rates and stellar tracks, and especially with different initial-final mass relationships, including the one derived in this work. Finally, we will perform a comparison of these results with the available observational data. 



\section{Chapter 2}

\section{White dwarfs in common proper motion pairs}

\section{$2.1 \quad$ Introduction}

The total age of a white dwarf can be expressed as $t=t_{\text {cool }}+t_{\text {prog }}$, where $t_{\text {cool }}$ is the cooling time of the white dwarf and $t_{\text {prog }}$ is the main-sequence lifetime of its progenitor. The cooling time of an isolated white dwarf can be derived using the proper cooling sequences. However, it is impossible to infer $t_{\text {prog }}$ since we do not know the mass of the progenitor or its metallicity. This problem can be avoided by studying white dwarfs with some known parameters. This is the case of white dwarfs in stellar clusters or in non-interacting binaries. Open or globular clusters are thought to be formed in a short burst of star formation (Kalirai et al. 2001). Hence, all the stars belonging to a cluster had the same initial chemical composition and are located at the same distance. Something similar occurs for white dwarfs in common proper motion pairs. It is sound to assume that the members of a common proper motion pair were born simultaneously and with the same chemical composition (Oswalt et al. 1988; Wegner 1973). Since the components are well separated (100 to $1000 \mathrm{AU}$ ), mass exchange between them is unlikely and it can be considered that they have evolved as isolated stars. Thus, important information on the white dwarf, such as its total age or the metallicity of the progenitor, can be inferred from the study of the companion. In particular, if the companion is an F, G or K type star the metallicity can be derived with high accuracy from detailed spectral analysis. On the other hand, the total age of the white dwarf can be derived using different methods. In our case we have employed stellar isochrones when the star is moderatly evolved, or the X-ray luminosity, if the star is very close to the ZAMS. 


\section{FGK star}

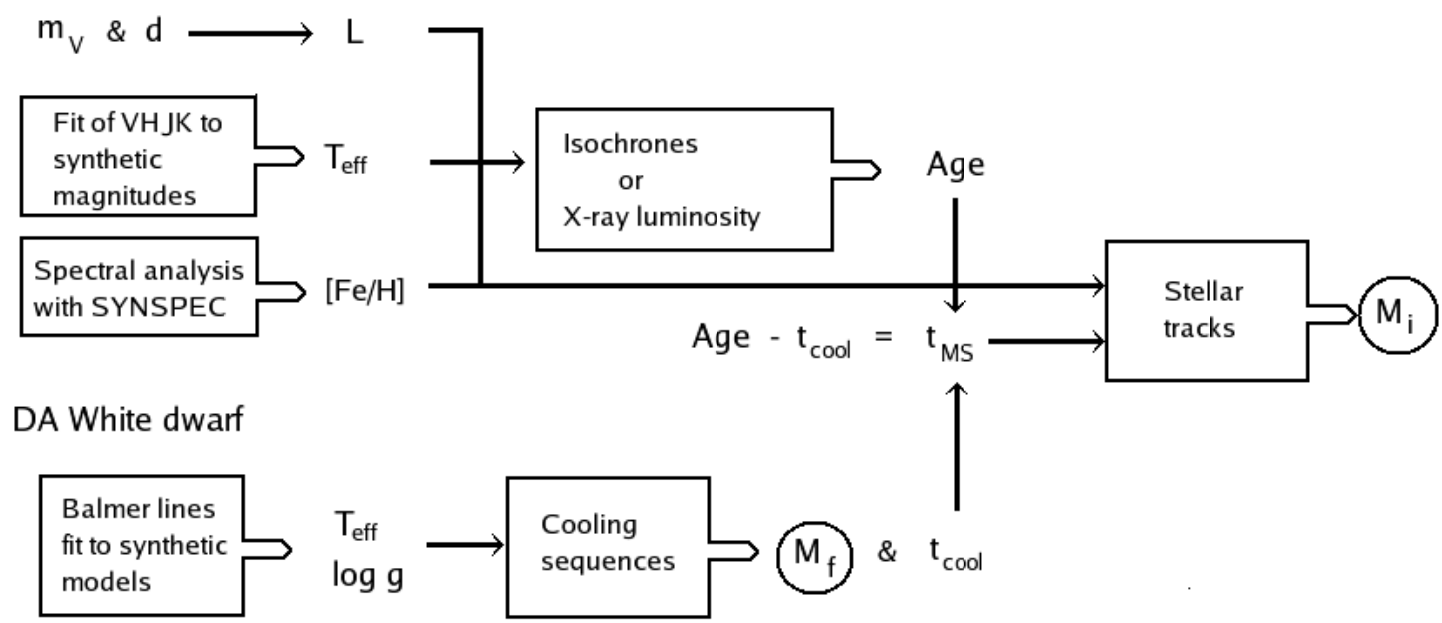

Figure 2.1: Scheme of the method used to infer the initial and final masses of white dwarfs in common proper motion pairs.

Common proper motion pairs containing white dwarfs have been intensively studied in the past. Oswalt (1981) performed spectroscopic observations of common proper motion binaries that may contain degenerate components, selecting the interesting targets from different catalogues (Luyten 1963; Giclas et al. 1971). Their work has been mainly focused on the pairs comprised of a white dwarf and an $\mathrm{M}$ dwarf. In Silvestri et al. (2005), they used the cooling sequences of white dwarfs to obtain a lower limit to the age of the M dwarf components. On the contrary, our purpose is to use the other member of the pair to calibrate the total age of the white dwarf, and also to infer the metallicity of its progenitor. The scheme of the method is shown in Fig. 2.1. This idea was first introduced by Wegner (1973). This author performed spectroscopic observations of some wide binaries in the southern hemisphere containing a white dwarf and a non-degenerate component with the main purpose of studying the mass-loss effects on the progenitors of white dwarfs. As a complementary analysis of his work, he also intended to calculate the initial masses of the white dwarfs deriving the total age and metallicity of the companions. However, the uncertainties were relatively high due to the low temperatures of the companions and their location in the main-sequence. The work described in this chapter is the first one using common proper motion pairs to determine the initial-final mass relationship. 
Table 2.1: Common proper motion pairs studied in this work.

\begin{tabular}{lllcc}
\hline \hline System & White Dwarf & Companion & Sp. type $^{1}$ & $\begin{array}{c}\text { Distance }^{1} \\
(\mathrm{pc})\end{array}$ \\
\hline G 158-78/77 & WD0023-109 & G158-77 & K & $\ldots$ \\
LP 592-80 & WD0315-011 & BD -01 469A & K1IV & $68.12 \pm 4.45$ \\
GJ 166 A/B & WD0413-077 & HD 26965 & K1V & $5.04 \pm 0.02$ \\
G 39-27/28 & WD0433+270 & BD +26 730 & K2 & $16.95 \pm 0.86$ \\
G 116-16/14 & WD0913+442 & BD +44 1847 & G0 & $51.65 \pm 3.47$ \\
G 163-B9B/A & WD1043-034 & G163-B9A & F9V & $\ldots$ \\
L P378-537 & WD1304+227 & BD +23 2539 & K0 & $\ldots$ \\
G 165-B5B/A & WD1354+340 & BD +34 2473 & F8 & $107.18 \pm 13.44$ \\
G 66-36/35 & WD1449+003 & G66-35 & G5V & $65.62 \pm 5.81$ \\
EGGR 113 & WD1544+008 & BD +01 3129 & G0 & $219.30 \pm 87.53$ \\
GJ 599 A/B & WD1544-377 & HD 140901 & G6V & $15.24 \pm 0.18$ \\
GJ 620.1 B/A & WD1620-391 & HD 147513 & G5V & $12.87 \pm 0.60$ \\
GJ 2125/GJ 3985 & WD1659-531 & HD 153580 & F6V & $26.33 \pm 0.58$ \\
G 140-B1B/ & WD1750+098 & BD +09 3501 & K0 & $\ldots$ \\
G 156-64/65 & WD2253-081 & BD -08 5980 & G6V & $34.82 \pm 1.58$ \\
\hline \hline
\end{tabular}

${ }^{1}$ From SIMBAD database.

\subsection{Observations and data reduction}

The sample of common proper motion pairs to be observed was chosen from the available literature, mainly from the papers of Silvestri et al. (2001) and Wegner \& Reid (1991), and from a cross-correlation of the SIMBAD database and the Villanova White Dwarf Catalog. We selected the pairs taking into account several requirements. Firstly, the white dwarf component should be classified as a DA (i.e., with the unique presence of Balmer lines), so that the fitting procedure is sufficiently accurate to derive realistic values for the effective temperature and surface gravity. Secondly, the other component of the pair should be a star of spectral type F, G or $\mathrm{K}$ for an accurate determination of the metallicity. The complete list of the studied targets is given in Table 2.1. The distance of each pair has been calculated considering the parallaxi given by the SIMBAD database. We also give some information about their proper motions in Table 2.2.

The observations were carried out during different campaigns between the summer of 2005 and the spring of 2007. In Table 2.3 we give details of the telescopeinstrument configurations employed, as well as the resolution and spectral coverage of each setup. Some of the white dwarf members were observed with the LCS spec- 
Table 2.2: Proper motions of the pairs studied in this work.

\begin{tabular}{|c|c|c|c|c|c|c|}
\hline System & White Dwarf & $\begin{array}{l}\mu_{\alpha} \\
(\mathrm{ma}\end{array}$ & $\begin{array}{c}\mu_{\delta}^{1} \\
/ \mathrm{yr})\end{array}$ & Companion & $\begin{array}{l}\mu_{\alpha} \\
\quad \text { mas }\end{array}$ & $\begin{array}{c}\mu_{\delta}^{1} \\
/ \mathrm{yr})\end{array}$ \\
\hline G $158-78 / 77$ & WD0023-109 & 72 & -205 & G158-77 & 73.6 & -206 \\
\hline LP 592-80/ & WD0315-011 ${ }^{2}$ & 254 & -112 & $\mathrm{BD}-01469 \mathrm{~A}^{2}$ & 254 & -112 \\
\hline GJ $166 \mathrm{~A} / \mathrm{B}$ & WD0413-077 & -2228 & -3377 & HD 26965 & -2239 & -3420 \\
\hline G $39-27 / 28$ & WD0433+270 & 228 & -155 & $\mathrm{BD}+26730$ & 232 & -147 \\
\hline G $116-16 / 14$ & WD0913+442 & 7 & -267 & $\mathrm{BD}+441847$ & 34 & -279 \\
\hline G $163-\mathrm{B} 9 \mathrm{~B} / \mathrm{A}^{3}$ & WD1043-034 & 72 & -34 & G163-B9A & & \\
\hline LP $378-537$ & WD1304+227 & -101 & 79 & $\mathrm{BD}+232539$ & -101 & 79 \\
\hline G $165-B 5 B / A$ & WD1354+340 & -139 & 12 & $\mathrm{BD}+342473$ & -156 & 50 \\
\hline G $66-36 / 35$ & WD1449+003 & 39 & -317 & G66-35 & -37 & -294 \\
\hline EGGR $113 /^{3}$ & WD1544+008 & $\ldots$ & $\ldots$ & $\mathrm{BD}+013129$ & -33 & 19 \\
\hline GJ 599 A/B & WD1544-377 & -415 & -214 & HD 140901 & -417 & -214 \\
\hline GJ 620.1 B/A & WD1620-391 & 75 & 0 & HD 147513 & 73 & 3 \\
\hline GJ 2125/GJ 3985 & WD1659-531 & 12 & -133 & HD 153580 & 1 & -165 \\
\hline G $140-B 1 B /$ & WD1750+098 & 10 & -100 & $\mathrm{BD}+093501$ & -5 & -157 \\
\hline G $156-64 / 65$ & WD2253-081 & 547 & -56 & $\mathrm{BD}-085980$ & 553 & -50 \\
\hline
\end{tabular}

${ }^{1}$ From SIMBAD database.

${ }^{2}$ Proper motion according to Luyten (1979).

${ }^{3}$ No proper motion values have been found for one of the members of this pair, although they were classified as a common proper motion pair.

${ }^{4}$ Proper motion according to McCook \& Sion (1999).

${ }^{5}$ Proper motion according to Farihi et al. (2005).

trograph of the Harlan J. Smith $(2.7 \mathrm{~m})$ telescope at McDonald Observatory (Texas, USA) covering the main Balmer lines (from $\mathrm{H}_{\beta}$ to $\mathrm{H}_{8}$ ) and obtaining a resolving power of $\sim 5 \AA$ FWHM. The fall targets were observed with the TWIN spectrograph of the $3.5 \mathrm{~m}$ telescope at Calar Alto Observatory (CAHA, Almería, Spain). In this case, the beam splitter of the TWIN spectrograph allowed us to cover both the blue and the red part of the Balmer lines (from 3570 to $5750 \AA$ in the blue range, and from 5700 to $7800 \AA$ in the red one), obtaining a resolving power of $\sim 4 \AA$ FWHM. WD0315-011 was kindly observed for us by T. Oswalt with the RC spectrograph at the $4 \mathrm{~m}$ telescope at Kitt Peak National Observatory obtaining $\sim 1.5 \AA$ FWHM. We performed as many exposures as necessary to guarantee a high signal-to-noise ratio final spectrum for each object, after the corresponding reduction. Spectra of high quality are essential to derive the atmospheric parameters with accuracy. We co-added individual $1800 \mathrm{~s}$ exposures to minimize the effects of cosmic ray impacts on the CCD. The FGK companions were observed with echelle spectro- 
Table 2.3: Journal of observations.

\begin{tabular}{lcccc}
\hline \hline Observatory & Telescope & Spectrograph & $\mathrm{R}$ & $\begin{array}{c}\text { Spectral } \\
\text { Coverage }\end{array}$ \\
\hline \multicolumn{5}{c}{ White Dwarfs } \\
\hline McDonald & $2.7 \mathrm{~m}$ HJS & LCS & 1000 & $3885-5267 \AA$ \\
CAHA & $3.5 \mathrm{~m}$ & TWIN & 1250 & $3570-5750 \AA$ \\
LCO & $6.5 \mathrm{~m}$ Clay & LDSS3 & 1650 & $3600-6000 \AA$ \\
\hline \multicolumn{5}{c}{ Low-mass Companions } \\
CAConald & $2.7 \mathrm{~m} \mathrm{HJS}$ & 2dCoudé & 60000 & $3400-10900 \AA$ \\
ORM & $2.2 \mathrm{~m}$ & FOCES & 47000 & $3600-9400 \AA$ \\
LCO & $3.5 \mathrm{~m}$ TNG & SARG & 57000 & $4960-10110 \AA$ \\
\hline \hline
\end{tabular}

graphs, obtaining high signal-to-noise high-resolution spectra $(\mathrm{S} / \mathrm{N}>150)$, which are necessary to derive the metallicity with accuracy. Recently, we have been able to obtain spectra from some southern hemisphere targets. These observations were kindly carried out at Las Campanas Observatory by A. Z. Bonanos. The white dwarf spectra were obtained using the LDSS3 spectrograph at the $6.5 \mathrm{~m}$ Magellan telescope with a resolving power of $\sim 2.8 \AA$ FWHM. The companions were observed with the MIKE echelle spectrograph at the same telescope with high resolution $(R \sim 50000)$.

The white dwarf spectra were reduced using the standard procedures within the single-slit tasks in IRAF ${ }^{1}$. First, the images were bias- and flatfield-corrected, and then, the spectra were extracted and wavelength calibrated using arc lamp observations. We combined multiple spectra of the same star to achieve a final spectrum of high signal-to-noise ratio ( $\mathrm{S} / \mathrm{N}>100)$. Before this step, we applied the heliocentric correction to each spectrum using the tasks rvcorrect and dopcor, since we were co-adding spectra secured in different days. Finally, the spectra were normalized to the continuum. For the reduction of the FGK stars spectra the procedure followed was similar to the case of white dwarfs but we used the corresponding echelle tasks in IRAF. In this case, we used the task apscatter in order to characterize and subtract the scattered light, which is typical from cross-dispersed echelle setups.

\footnotetext{
${ }^{1}$ IRAF is distributed by the National Optical Astronomy observatories, which are operated by the Association of Universitites for Research in Astronomy, Inc., under cooperative agreement with the national Science Foundation (http://iraf.noao.edu).
} 


\subsection{White dwarf analysis}

\subsubsection{Classification}

After the corresponding reduction, we carried out a first inspection of the spectra. All the objects in Table 2.4 were eventually classified as DA white dwarfs. However, we found that four of them are not of the DA type. Particularly, WD1750+098 turned out to be of type DC although in the last reference in the literature (Silvestri et al. 2001) it was classified as a DA. We believe that WD1544+008 is the same star as WD1544+009, which was classified as a DAB white dwarf by Silvestri et al. (2001). However, it was identified as a sdO star by Wegner \& Reid (1991). The same authors also studied WD1043-034 and classified it as a sdB star, although McCook \& Sion (1999) considered it as a DAB white dwarf. Taking into account the different inconsistencies in the literature we decided to reobserve these objects in order to revise their spectral classifications if necessary. The reduced spectra of these two stars were kindly analyzed by P. Bergeron, who performed the corresponding fits and derived their temperatures and surface gravities, which turned out to be too low to be white dwarfs. As can be seen in Table 2.4, WD1449+003 is an M star. This classification was also recently indicated by Farihi et al. (2005), who also indicated that these stars had been wrongly identified as companions considering the difference between theire proper motions (see Table 2.2). Farihi et al. (2005) also reported that WD0913+442 and BD +44 1847 are not a physical pair according to their parallaxes. It is worth mentioning that some of the previous misclassifications are probably due to the fact that the signal-to-noise ratio of the spectra used was low.

The spectrum of WD0433+270 shows weak absorption lines but we were able to unambiguously identify $\mathrm{H}_{\beta}$. The weakness of the lines is the reason why this star had been previously classified as a type DC white dwarf by several authors (Eggen \& Greenstein 1965; Oswalt \& Strunk 1994). Higher resolution observations by Bergeron et al. (2001) and Zuckerman et al. (2003) had permitted the identification of $\mathrm{H}_{\alpha}$ and $\mathrm{H}_{\beta}$, respectively. Putting all results together we can confidently classify this object as a DA white dwarf. As reported in Catalán et al. (2008b), the system formed by WD0433+270 and BD +26 730 is somewhat peculiar, so, we will discuss it separately in the next chapter.

\subsubsection{Atmospheric parameters}

Before calculating the atmospheric parameters of the white dwarfs $\left(T_{\text {eff }}\right.$ and $\left.\log g\right)$ we determined the radial velocities of each star using the IRAF task fxcor. Each spectrum was cross-correlated with a reference model from a grid computed by D. Koester (private communication). The obtained radial velocities, estimated with large error bars, were generally small (ranging from 10 to $50 \mathrm{~km} / \mathrm{s}$ ) compared with the resolution element $(300 \mathrm{~km} / \mathrm{s})$ of our observations. In only one case (WD0023+109), the 
Table 2.4: Spectral classification of the white dwarfs.

\begin{tabular}{|c|c|c|c|}
\hline Name & This work & Previous & Reference \\
\hline WD0023-109 & DA & DA & EG65, WR91, OS94, MS99 \\
\hline WD0315-011 & DA & DA & OS94, MS99, SOW01 \\
\hline \multirow[t]{2}{*}{ WD0413-077 } & DA & DA & EG65, FKB97, MS99, HOS02 \\
\hline & & DA & HBB03, KNH05, HB06 \\
\hline \multirow[t]{2}{*}{ WD0433+270 } & DA & $\mathrm{DA}$ & GL90, BLR01, ZKR03 \\
\hline & & $\mathrm{DC}$ & EG65, OS94 \\
\hline \multirow[t]{2}{*}{ WD0913+442 } & DA & DA & EG65, WR91, BLF95, BLR01 \\
\hline & & DA & ZKR03, KNH05, LBH05, HB06 \\
\hline \multirow[t]{2}{*}{ WD1043-034 } & $\operatorname{sdB}^{1}$ & $\mathrm{DA} / \mathrm{sd}$ & WR91 \\
\hline & & $\mathrm{DAB}$ & OS94, MS99 \\
\hline WD1304+227 & DA & DA & O81, OS94, MS99, SOW01 \\
\hline WD1354+340 & $\mathrm{DA}$ & $\mathrm{DA}$ & EG67, WR91, BLF95, MS99, SOW01 \\
\hline \multirow{2}{*}{ WD1449+003 } & $\mathrm{M}$ & DA & O81, WR91, OS94 \\
\hline & & M & FBZ05 \\
\hline \multirow[t]{3}{*}{ WD1544+008 } & $\mathrm{sdO}^{1}$ & $\mathrm{DA} / \mathrm{sdO}$ & W91 \\
\hline & & $\mathrm{DA}$ & EG65, MS99 \\
\hline & & DAB & SOW01 \\
\hline \multirow[t]{3}{*}{ WD1544-377 } & DA & DA & EG65, W73, OS94, PSH98 \\
\hline & & DA & BLR01, KNC01, SOW01, HOS02 \\
\hline & & DA & HBB03, ZKR03, KNH05, KVS07 \\
\hline \multirow[t]{2}{*}{ WD1620-391 } & DA & DA & W73, OS94, HBS98, PSH98, SOW01 \\
\hline & & $\mathrm{DA}$ & HOS02, HBB03, HB06, KVS07 \\
\hline WD1659-531 & DA & DA & W73, OS94, PSH98, SOW01, KVS07 \\
\hline \multirow[t]{2}{*}{ WD1750+098 } & $\mathrm{DC}$ & DA & WR91, SOW01 \\
\hline & & $\mathrm{DC}$ & EG65, OS94, MS99 \\
\hline \multirow[t]{2}{*}{ WD2253-081 } & DA & DA & OS94, BLF95, MS99, BLR01 \\
\hline & & DA & KNC01, SOW01, KNH05 \\
\hline
\end{tabular}

${ }^{1} \mathrm{P}$. Bergeron, private communication.

BLF95: Bergeron et al. (1995); BLR01: Bergeron et al. (2001); EG65: Eggen \& Greenstein (1965); EG67: Eggen \& Greenstein (1967); FBZ05: Farihi et al. (2005); FKB97: Finley et al. (1997); GL90: Greenstein \& Liebert (1990); HOS02: Holberg et al. (2002); HBB03: Holberg et al. (2003); HB06: Holberg \& Bergeron (2006); KNC01: Koester et al. (2001); KNH05: Karl et al. (2005); KVS07: Kawka et al. (2007); MS99: McCook \& Sion (1999); O81: Oswalt (1981); OS94: Oswalt \& Strunk (1994); PSH98: Provencal et al. (1998); SOW01: Silvestri et al. (2001); W73: Wegner (1973); WR91: Wegner \& Reid (1991); ZKR03: Zuckerman et al. (2003) 
Table 2.5: Atmospheric parameters derived for the observed white dwarfs.

\begin{tabular}{lcc}
\hline \hline Name & $T_{\text {eff }}(K)$ & $\log g($ dex $)$ \\
\hline WD0023+109 & $10380 \pm 230$ & $7.92 \pm 0.08$ \\
WD0315-011 & $7520 \pm 260$ & $8.01 \pm 0.45$ \\
WD0413-077 & $16570 \pm 350$ & $7.86 \pm 0.05$ \\
WD0913+442 & $8920 \pm 110$ & $8.29 \pm 0.10$ \\
WD1304+227 & $10800 \pm 120$ & $8.21 \pm 0.05$ \\
WD1354+340 & $13650 \pm 420$ & $7.80 \pm 0.15$ \\
WD1544-377 & $10600 \pm 250$ & $8.29 \pm 0.05$ \\
WD1620-391 & $24900 \pm 130$ & $7.99 \pm 0.03$ \\
WD1659-531 & $14510 \pm 250$ & $8.08 \pm 0.03$ \\
WD2253-081 & $7220 \pm 140$ & $8.25 \pm 0.20$ \\
\hline \hline
\end{tabular}

${ }^{1}$ We do not have a spectrum of this star. These values are from Heber et al. (1997) and Bergeron et al. (1995).

radial velocity measured turned out to be relevant $(150 \mathrm{~km} / \mathrm{s})$. However, all radial velocities were taken into account for consistency.

After this previous step, we derived the atmospheric parameters of these stars performing a fit of the observed Balmer lines to white dwarf models following the procedure described in Bergeron et al. (1992). The models had been previously normalized to the continuum and convolved with a Gaussian instrumental profile with the proper FWHM in order to have the same resolution as the observed spectra. The fit of the line profiles was then carried out using the task specfit of the IRAF package, which is based on $\chi^{2}$ minimization considering the non-linear least-squares Levenberg-Marquardt method (Press et al. 1992). We used specfit for different $\log g$ values $(7.0,7.5,8.0,8.5$ and 9.0$)$ with $T_{\text {eff }}$ as a free parameter, obtaining different $\chi^{2}$ for each fit. In each case, the initial estimate for $T_{\text {eff }}$ obtained from the spectral energy distribution (photometry in the $B V$ and $J H K$ bands, 2MASS) was used as a starting guess. The final $T_{\text {eff }}$ was derived by minimizing the reduced $\chi^{2}$, $\chi_{\nu}^{2}$, considering the values yielding $\chi_{\nu}^{2} \pm 1$ to estimate the errors. $\chi_{\nu}^{2}$ can be defined as:

$$
\chi_{\nu}^{2}=\frac{\chi^{2}}{N_{p}+n-1},
$$

where $N_{p}$ is the number of points used in the fit and $n$ is the number of free parameters. 
On the other hand, $\chi^{2}$ is defined as:

$$
\chi^{2}=\sum_{i=1}^{n} \frac{\left(f_{\mathrm{obs}}-f_{\mathrm{mod}}\right)_{i}^{2}}{\sigma_{i}^{2}} .
$$

where $f_{\text {obs }}$ are the observed relative flux and $f_{\text {mod }}$ the values corresponding to the synthetic models.

The determination of $\log g$ was performed in an analogous way but to calculate the errors we took into account the prescription of Bergeron et al. (1992), who derive them from the independent fits of each spectrum of a given star (before the combination). The results of these fits are given in Table 2.5. In Fig. 2.2 we show the fits for some of the DA white dwarfs in our sample to illustrate the goodness of our fitting procedure. The solid lines correspond to the observed spectra, while the dashed lines are synthetic models.

Some of these white dwarfs had been the subject of previous analyses which allow us to perform a comparison with our results. For instance, WD0913+442 was also studied by Bergeron et al. (1995), who obtained atmospheric parameters compatible with the ones derived here. They also studied WD1354+340 and WD2253-081, but in these cases the effective temperatures obtained are compatible with ours while the surface gravities are not, although just outside the $1 \sigma$ error bar. We have obtained lower values of $\log g$ in both cases, which could be due to the different resolution of the spectra ( $\sim 6 \AA$ FHWM in their case). This latter object, WD2253-081, is of particular interest since an accurate fit of its line profiles posed many problems to previous analyses because the lines seemed to be broader than the models predicted. This led different authors to consider the possibility of this star to be a magnetic white dwarf or to have its lines rotationally broadened. Both options were considered by Karl et al. (2005), who discarded the former possibility. With the purpose of solving the fitting problem of this star, in this work we have used updated models for DA white dwarfs with effective temperatures between 6000 and $10000 \mathrm{~K}$. These models were kindly provided by D. Koester, who calculated them considering collision-induced absorption due to the presence of molecular hydrogen. This effect is very significant at low temperatures and it should be taken into account for an accurate determination of the atmospheric parameters. Contrarily to the results obtained by Karl et al. (2005) we did not need to consider rotational broadening to achieve a good fit. On the other hand, the southern hemisphere targets had been also studied by different authors. Recently, Kawka et al. (2007) derived the atmospheric parameters of WD1544-377, WD1620-391 and WD1659-531, which are in good agreement with our results. 

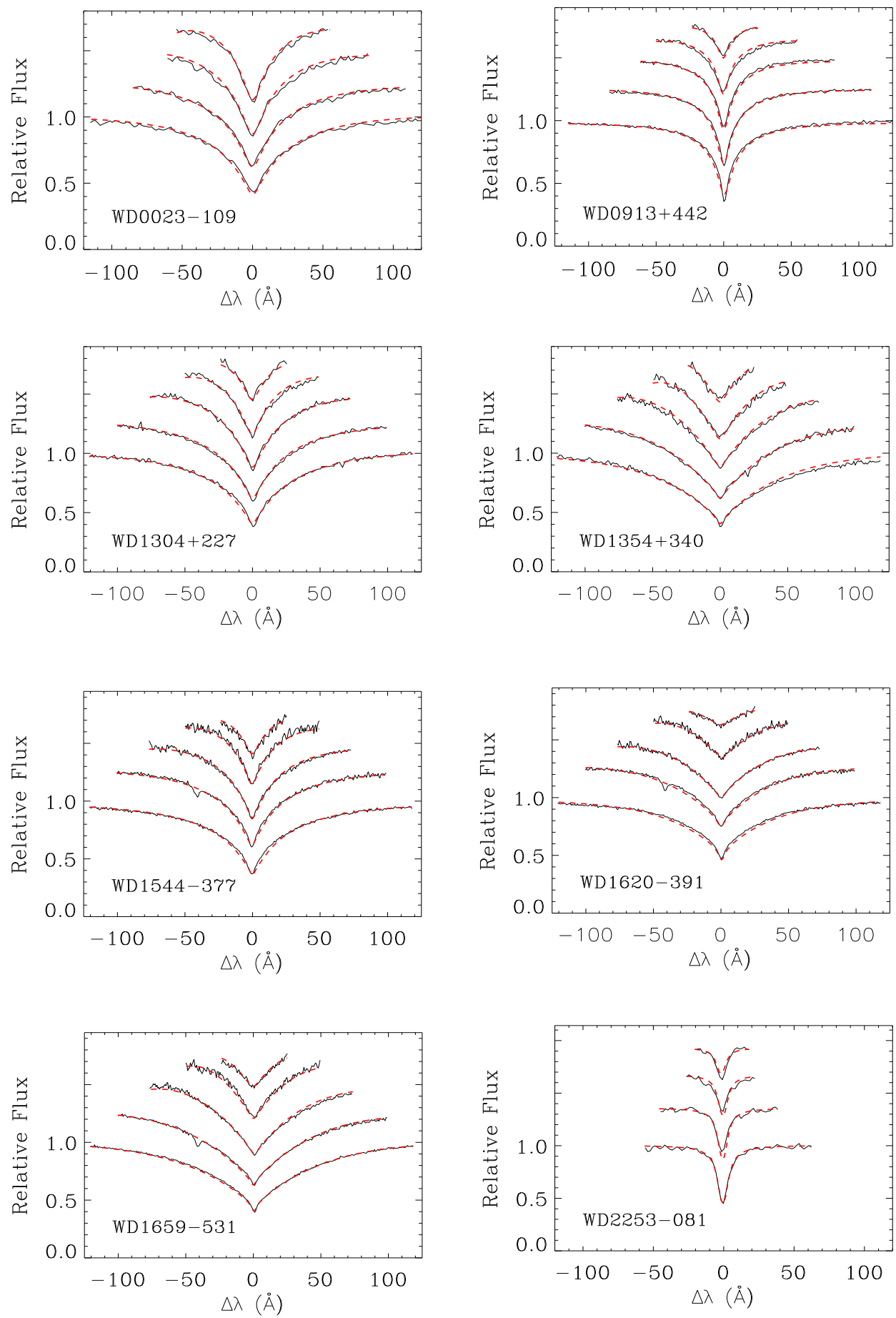

Figure 2.2: Fits of the observed Balmer lines for the white dwarfs studied here. Dashed lines correspond to synthetic models. Balmer lines range from $\mathrm{H}_{\beta}$ (bottom) to $\mathrm{H}_{8}$ (top), except for WD0023-109 and WD2253-081 (to $\mathrm{H}_{\varepsilon}$ ). 


\subsubsection{Masses and cooling times}

Once we have derived the $T_{\text {eff }}$ and $\log g$ of each star, we can obtain its mass ( $M_{\mathrm{WD}}$ ) and cooling time $\left(t_{\text {cool }}\right)$ from appropriate cooling sequences. We have used the cooling tracks of Salaris et al. (2000) - model S0 - which consider a carbon-oxygen $(\mathrm{C} / \mathrm{O})$ core white dwarf (with a higher abundance of $\mathrm{O}$ at the center of the core) surrounded by a $\mathrm{H}$ thick envelope on top of a He buffer, $q(\mathrm{H})=M_{\mathrm{H}} / M=10^{-4}$ and $q(\mathrm{He})=M_{\mathrm{He}} / M=10^{-2}$. These improved cooling sequences include an accurate treatment of the crystallization process of the $\mathrm{C} / \mathrm{O}$ core, including phase separation upon crystallization, together with up-to-date input physics suitable for computing white dwarf evolution. On the other hand, and in order to be able to check the sensitivity of our results to the adopted cooling tracks, we have also used the cooling sequences of Fontaine et al. (2001) with different core compositions. In a first series of calculations $\mathrm{C} / \mathrm{O}$ cores with a composition of $50 / 50$ by mass with thick $\mathrm{H}$ envelopes, $q(\mathrm{H})=10^{-4}$, on top of a He buffer, $q(\mathrm{He})=10^{-2}$, were adopted. We refer to these models as F0. In the second series of calculations cooling sequences with a pure $\mathrm{C}$ core and the same envelope characteristics - model F1 - were used. As can be seen in Table 2.6, the derived masses do not change appreciably when adopting different cooling sequences. On the contrary, small differences can be noted in the cooling times obtained, depending on the evolutionary tracks used. This stems naturally from the different core compositions of the cooling sequences adopted here. As can be noted by examining Table 2.6, considering a $\mathrm{C} / \mathrm{O}$ core with equal carbon-oxygen mass fractions with thick envelopes (model F0) is quite similar to considering a $\mathrm{C} / \mathrm{O}$ core with more $\mathrm{O}$ concentrated in the center of the core (model S0) in terms of the cooling time. Also, and as it should be expected, we obtain larger values for the cooling times when considering the pure $\mathrm{C}$ core sequences (model F1), since a white dwarf with a pure $\mathrm{C}$ core cools slower than a white dwarf with a $\mathrm{C} / \mathrm{O}$ core because of the higher heat capacity of $\mathrm{C}$ in comparison with that of $\mathrm{O}$, implying a larger amount of energy necessary to change the temperature of the core. More details on the adopted sets of cooling tracks are given in appendix A.

Some of these white dwarfs have mass estimates from previous investigations. Silvestri et al. (2001) calculated masses from gravitational redshifts for WD0315-011, WD1354+340, WD1544-377, WD1620-391, WD1659-531 and WD2253-081. The results of that study are compatible with the masses derived in this work except for WD1544-377, whose mass is $25 \%$ smaller when calculated from its gravitational redshift. However, Kawka et al. (2007) inferred the spectroscopic mass of this star, together with those of WD1620-391 and WD1659-531, that are in good agreement with our results. WD0913+442 was studied by Karl et al. (2005) and Bergeron et al. (2001). The former inferred the spectroscopic mass of the white dwarf and the latter used photometry and trigonometric parallax to estimate the mass. In both cases, the results are compatible with the value derived here. 
Table 2.6: Stellar parameters derived for the observed white dwarfs.

\begin{tabular}{ccccccc}
\hline \hline & \multicolumn{2}{c}{ Model S0 } & \multicolumn{2}{c}{ Model F0 } & \multicolumn{2}{c}{ Model F1 $^{\mathrm{b}}$} \\
\hline Name & $\begin{array}{c}M_{\mathrm{WD}} \\
\left(M_{\odot}\right)\end{array}$ & $\begin{array}{c}t_{\text {cool }} \\
(\mathrm{Gyr})\end{array}$ & $\begin{array}{c}M_{\mathrm{WD}} \\
\left(M_{\odot}\right)\end{array}$ & $\begin{array}{c}t_{\text {cool }} \\
(\mathrm{Gyr})\end{array}$ & $\begin{array}{c}M_{\mathrm{WD}} \\
\left(M_{\odot}\right)\end{array}$ & $\begin{array}{c}t_{\text {cool }} \\
(\mathrm{Gyr})\end{array}$ \\
\hline WD0023-109 & $0.56 \pm 0.03$ & $0.47 \pm 0.03$ & $0.56 \pm 0.03$ & $0.50 \pm 0.03$ & $0.56 \pm 0.03$ & $0.53 \pm 0.03$ \\
WD0315-011 & $0.60 \pm 0.20$ & $1.20 \pm 0.56$ & $0.60 \pm 0.18$ & $1.28 \pm 0.45$ & $0.60 \pm 0.18$ & $1.37 \pm 0.42$ \\
WD0413-077 & $0.54 \pm 0.02$ & $0.112 \pm 0.008$ & $0.54 \pm 0.02$ & $0.11 \pm 0.01$ & $0.54 \pm 0.02$ & $0.12 \pm 0.01$ \\
WD0913+442 & $0.78 \pm 0.01$ & $1.24 \pm 0.05$ & $0.78 \pm 0.05$ & $1.24 \pm 0.15$ & $0.78 \pm 0.05$ & $1.35 \pm 0.12$ \\
WD1304+227 & $0.73 \pm 0.02$ & $0.62 \pm 0.03$ & $0.73 \pm 0.02$ & $0.68 \pm 0.03$ & $0.73 \pm 0.02$ & $0.71 \pm 0.03$ \\
WD1354+340 & $0.50 \pm 0.04$ & $0.20 \pm 0.02$ & $0.50 \pm 0.04$ & $0.19 \pm 0.02$ & $0.50 \pm 0.03$ & $0.21 \pm 0.02$ \\
WD1544-377 & $0.78 \pm 0.02$ & $0.76 \pm 0.05$ & $0.78 \pm 0.02$ & $0.81 \pm 0.04$ & $0.78 \pm 0.02$ & $0.86 \pm 0.05$ \\
WD1620-391 & $0.63 \pm 0.01$ & $0.026 \pm 0.001$ & $0.63 \pm 0.01$ & $0.022 \pm 0.001$ & $0.63 \pm 0.01$ & $0.025 \pm 0.001$ \\
WD1659-531 & $0.66 \pm 0.01$ & $0.24 \pm 0.01$ & $0.66 \pm 0.01$ & $0.25 \pm 0.01$ & $0.66 \pm 0.01$ & $0.26 \pm 0.01$ \\
WD2253-081 & $0.75 \pm 0.09$ & $2.32 \pm 0.72$ & $0.75 \pm 0.09$ & $2.20 \pm 0.44$ & $0.75 \pm 0.09$ & $2.27 \pm 0.53$ \\
\hline \hline
\end{tabular}

a S0 Salaris et al. (2000)

b F0, F1 Fontaine et al. (2001) 
Table 2.7: Photometric information available for the observed FGK stars and the effective temperatures derived.

\begin{tabular}{lccccc}
\hline \hline Name & $V^{1}$ & $J$ & $H$ & $K$ & $T_{\text {eff }}(\mathrm{K})$ \\
\hline G 158-77 & $12.900 \pm 0.020$ & $10.740 \pm 0.024$ & $10.135 \pm 0.022$ & $9.934 \pm 0.019$ & $4390 \pm 30$ \\
BD -01 469A & $5.370 \pm 0.020$ & $3.404 \pm 0.198$ & $2.818 \pm 0.210$ & $2.678 \pm 0.234$ & $4480 \pm 50$ \\
HD 26965 & $4.410 \pm 0.020$ & $3.013 \pm 0.238$ & $2.594 \pm 0.198$ & $2.498 \pm 0.236$ & $5160 \pm 35$ \\
BD +44 1847 & $9.000 \pm 0.020$ & $7.685 \pm 0.019$ & $7.389 \pm 0.017$ & $7.315 \pm 0.021$ & $5630 \pm 50$ \\
BD +23 2539 & $9.710 \pm 0.020$ & $8.458 \pm 0.023$ & $8.109 \pm 0.016$ & $8.074 \pm 0.017$ & $5665 \pm 50$ \\
BD +34 2473 & $9.080 \pm 0.020$ & $8.090 \pm 0.030$ & $7.851 \pm 0.034$ & $7.836 \pm 0.024$ & $6270 \pm 70$ \\
HD 140901 & $6.010 \pm 0.020$ & $4.959 \pm 0.214$ & $4.505 \pm 0.076$ & $4.323 \pm 0.016$ & $5635 \pm 50$ \\
HD 147513 & $5.376 \pm 0.020$ & $4.405 \pm 0.258$ & $4.025 \pm 0.190$ & $3.933 \pm 0.036$ & $5985 \pm 75$ \\
HD 153580 & $5.285 \pm 0.020$ & $4.453 \pm 0.318$ & $4.181 \pm 0.206$ & $4.126 \pm 0.036$ & $6470 \pm 90$ \\
BD -08 5980 & $8.030 \pm 0.020$ & $6.816 \pm 0.034$ & $6.492 \pm 0.033$ & $6.353 \pm 0.020$ & $5670 \pm 50$ \\
\hline \hline
\end{tabular}

${ }^{1}$ Magnitude errors adopted.

${ }^{2} T_{\text {eff }}$ derived from the Eggen $R I$ photometry using the color- $T_{\text {eff }}$ relation from Houdashelt et al. (2000). JHK 2MASS magnitudes were saturated.

${ }^{3} T_{\text {eff }}$ derived from the $V-K$ color using the color-temperature relation from Masana et al. (2006). JHK 2MASS magnitudes were saturated.

\subsection{Low-mass members analysis}

\subsubsection{Determination of $T_{\text {eff }}$}

We have used the available photometry $-V$ from SIMBAD and $J H K$ from 2MASS, as shown in Table 2.7 - to derive the effective temperatures of these stars, $T_{\text {eff }}$, following the method of Masana et al. (2006). This procedure consists in calculating synthetic photometry using the non-overshoot Kurucz atmosphere model grid $^{2}$. Masana et al. (2006) developed a fitting algorithm that is based on the minimization of the $\chi^{2}$ parameter using the Levenberg-Marquardt method (Press et al. 1992). As usual, $\chi^{2}$ is defined from the differences between the observed and synthetic VHJK magnitudes:

$$
\begin{aligned}
\chi^{2} & =\left(\frac{V-A_{V}-V_{\mathrm{syn}}}{\sigma_{V}}\right)^{2}+\left(\frac{J-A_{J}-J_{\mathrm{syn}}}{\sigma_{J}}\right)^{2} \\
& +\left(\frac{H-A_{H}-H_{\mathrm{syn}}}{\sigma_{H}}\right)^{2}+\left(\frac{K-A_{K}-K_{\mathrm{syn}}}{\sigma_{K}}\right)^{2}
\end{aligned}
$$

\footnotetext{
${ }^{2}$ http://kurucz.harvard.edu/grids.html
} 
Table 2.8: $R I$ Eggen photometry available for the stars with saturated 2MASS magnitudes.

\begin{tabular}{lcccccc}
\hline \hline Name & $(R-I)_{\mathrm{K}}$ & $(V-R)_{\mathrm{K}}$ & $(V-I)_{\mathrm{K}}$ & $(R-I)_{\mathrm{C}}$ & $(V-R)_{\mathrm{C}}$ & $(V-I)_{\mathrm{C}}$ \\
\hline BD -01 469A & 0.375 & 0.540 & 0.915 & 0.500 & 0.626 & 1.126 \\
HD 26965 & 0.305 & 0.340 & 0.645 & 0.432 & 0.441 & 0.873 \\
\hline \hline
\end{tabular}

This function depends indirectly on $T_{\text {eff }}, \log g,[\mathrm{Fe} / \mathrm{H}]$ and a magnitude difference $\Re$, which is the ratio between the synthetic and the observed flux at Earth, $\Re=-2.5 \log \left(F_{\text {star }} / F_{\text {earth }}\right)$. Tests show that the spectral energy distribution in the optical/IR for the range of temperatures that corresponds to FGK stars is only weakly dependent on gravity and metallicity, which makes it possible to derive accurate temperatures for stars with poor determinations of $\log g$ and $[\mathrm{Fe} / \mathrm{H}]$. Taking this into account, we assume initial values of $\log g=4.5$ and $[\mathrm{Fe} / \mathrm{H}]=0.0$ to estimate the effective temperatures. We did not consider interstellar extinction corrections, since they have negligible effects considering the nearby distances of the stars under study. The results are given in Table 2.7. As it can be seen the JHK 2MASS magnitudes for BD -01 469A and HD 26965 are saturated. Thus, in order to derive accurate effective temperatures for these stars, we considered the Eggen $R I$ photometry (Eggen 1971) available from the Lausanne Photometric Database (GCPD) see Table 2.8. We used the relations of Bessell (1979) to transform between Cousins and the Kron-Eggen system in order to obtain $(R-I)_{\mathrm{C}}$ and $(V-I)_{\mathrm{C}}$ from $(R-I)_{\mathrm{K}}$ and $(V-I)_{\mathrm{K}}$ :

$$
\begin{array}{lr}
(R-I)_{\mathrm{C}}=0.118+1.03(R-I)_{\mathrm{K}} & (R-I)_{\mathrm{K}}<0.35 \\
(R-I)_{\mathrm{C}}=0.033+1.246(R-I)_{\mathrm{K}} & 0.35< \\
(V-I)_{\mathrm{C}}=0.27+0.936(V-I)_{\mathrm{K}}<1.30 \\
(V-I)_{\mathrm{C}}=0.24+1.00(V-I)_{\mathrm{K}} & (V-I)_{\mathrm{K}}>0.5 \\
& (V-I)_{\mathrm{K}}<0.5
\end{array}
$$

Then, we consider the suitable color-temperature relations derived by Houdashelt et al. (2000) to infer their effective temperatures. For giants we adopt:

$$
\begin{array}{ll}
T_{\text {eff }}=8556.22-5235.57(V-I)_{\mathrm{C}}+1471.09(V-I)_{\mathrm{C}}^{2} & 0.70<V-I<1.68 \\
T_{\text {eff }}=8311.02-9145.75(V-R)_{\mathrm{C}}+4690.79(V-R)_{\mathrm{C}}^{2} & 0.37<V-R<0.86
\end{array}
$$


Table 2.9: Johnson photometry available for HD 26965.

\begin{tabular}{lccccc}
\hline \hline Name & $J$ & $H$ & $K$ & $L$ & $(V-K)_{J-G}$ \\
\hline HD 26965 & 2.95 & 2.48 & 2.41 & 2.37 & 2.005 \\
\hline \hline
\end{tabular}

Whereas for dwarfs we use:

$$
\begin{array}{ll}
T_{\text {eff }}=9058.78-6152.43(V-I)_{\mathrm{C}}+1987.84(V-I)_{\mathrm{C}}^{2} & 0.40<V-I<1.55 \\
T_{\text {eff }}=8757.63-10410.8(V-R)_{\mathrm{C}}+6078.04(V-R)_{\mathrm{C}}^{2} & 0.21<V-R<0.82
\end{array}
$$

In the case of BD $-01469 \mathrm{~A}$ we use the equations for giant stars, obtaining $T_{\text {eff }}=4525 \mathrm{~K}$ and $T_{\text {eff }}=4425 \mathrm{~K}$, from $(V-I)_{\mathrm{C}}$ and $(V-R)_{\mathrm{C}}$, respectively. At the same database there is also the Strömgren photometry available, $b-y=0.633$. Considering this value and the calibration of Olsen (1984):

$$
10^{3} \log T_{\text {eff }}=-0.341(b-y)+3869
$$

we obtain $T_{\text {eff }}=4499 \mathrm{~K}$. We consider as the final result the mean value of these three temperatures, $T_{\text {eff }}=4480 \pm 50 \mathrm{~K}$. The effective temperature obtained for $\mathrm{BD}-01469 \mathrm{~A}$ is $230 \mathrm{~K}$ lower than the one reported in McWilliam (1990), who used $(B-V)$ from the Bright Star Catalog (BSC) and the corresponding calibration of color-temperature. These authors derived the effective temperature from an extrapolation, since their calibration did not cover stars with such low temperatures. Thus, we consider that the value that we have obtained is more reliable.

Regarding HD 26965 we used the relations of Houdashelt et al. (2000) corresponding to dwarf stars, obtaining $T_{\text {eff }}=5200 \mathrm{~K}$ and $T_{\text {eff }}=5345 \mathrm{~K}$, from $(V-I)_{\mathrm{C}}$ and $(V-R)_{\mathrm{C}}$ respectively. Besides the Eggen $R I$ photometry, the $J$ and $K$ Johnson magnitudes (Johnson et al. 1968) of HD 26965 are also available at the Lausanne Photometric Database (GCPD). These photometric data are given in Table 2.9. We used the relation of Bessell \& Brett (1988):

$$
(V-K)_{\mathrm{J}}=-0.005+(V-K)_{\mathrm{J}-\mathrm{G}}
$$

to transform $V-K$ from the Johnson to the Johnson-Glass system. Then, we used the $(V-K)$-temperature calibration of Houdashelt et al. (2000):

$$
T_{\text {eff }}=8686.22-2441.65(V-K)+334.789(V-K)^{2} \quad 0.82<V-K<3.29
$$


for dwarfs, obtaining $T_{\text {eff }}=5135 \mathrm{~K}$. We can also use the $(V-K)$-temperature relation from Masana et al. (2006):

$$
\begin{aligned}
\frac{5040}{T_{\text {eff }}} & =0.5135+0.2687(V-K)-0.0174(V-K)^{2} \\
& +0.0298[\mathrm{Fe} / \mathrm{H}]-0.0009[\mathrm{Fe} / \mathrm{H}]^{2} \\
& -0.0184(V-K)[\mathrm{Fe} / \mathrm{H}]-0.0028 \log g
\end{aligned}
$$

which is valid for $1.15<V-K<3.20$. This gives $T_{\text {eff }}=5185 \mathrm{~K}$. We find the values derived from the $(V-K)$ color much more confident, so, our final value should be the mean of them, $T_{\text {eff }}=5160 \pm 35 \mathrm{~K}$. This value is in reasonable agreement with $T_{\text {eff }}=5090 \mathrm{~K}$, which is the result obtained by Steenbook (1983) using also the available $V-R, V-I, V-J, V-K$, and $V-L$ colors and the color calibrations of Johnson et al. (1966).

\subsubsection{Determination of $[\mathrm{Fe} / \mathrm{H}]$}

To derive the metallicity of the stars we fitted the observed absorption lines with synthetic spectra computed with SYNSPEC ${ }^{3}$ (Hubeny \& Lanz 1995) and the model atmospheres of Kurucz (1979). For each star, we used the model corresponding to the derived $T_{\text {eff }}$ and assumed a value for $\log g$. SYNSPEC is a program for calculating the spectrum emergent from a given model atmosphere. It was originally designed to synthesize spectra from atmospheres calculated using TLUSTY (Lanz \& Hubeny 1995), but may also be used with other model atmospheres as input (e.g., LTE ATLAS models of Kurucz, as in our case). The program is complemented by the routine rotins that calculates the rotational and instrumental convolutions for the net spectrum produced by SYNSPEC. The technique used to synthesize the spectra is based upon the weak line aproximation. The relationship between the equivalent width and the optical depth is relatively well known near the surface, where it is linear, but tends to become more complicated as the atmosphere becomes deeper. Hence, high resolution spectra are needed to obtain good results in the fitting procedure.

Line selection and atomic data calibration is a crucial step to derive the metallicity of a star. We selected the lines from two sources: Reddy et al. (2003) and Ramírez et al. (2007) taking into account different requirements. The suitable stellar lines should have a relatively small equivalent width, i.e., $\Delta W_{\lambda}<50 \mathrm{~m} \AA$ approximately. We rejected those lines with very low excitation potential transitions in order to avoid possible non-LTE effects. We discarded also the lines which fell in the spectral gaps between the spectral orders or those that appeared asymmetric, which were assumed to be blended with unidentified lines. It is very important also to consider

\footnotetext{
${ }^{3}$ http://nova.astro.umd.edu/Synspec43/synspec.html
} 
Table 2.10: Solar lines chosen to calibrate the atomic data list. The values of $\log g f$ used by different authors and the ones determined in this work are also given, as well as the equivalent widths of these lines in the solar spectrum.

\begin{tabular}{|c|c|c|c|c|c|c|c|c|}
\hline Species & $\begin{array}{l}\text { Wavelength } \\
(\AA)\end{array}$ & $\begin{array}{l}\mathrm{LEP} \\
(\mathrm{eV})\end{array}$ & $\begin{array}{l}\log g f \\
\text { Original }\end{array}$ & $\begin{array}{l}\log g f \\
\text { RAL }\end{array}$ & $\begin{array}{l}\log g f \\
\text { RTLA }\end{array}$ & $\begin{array}{c}\log g f \\
\text { AMMSF }\end{array}$ & $\begin{array}{l}\log g f \\
\text { Adopted value }\end{array}$ & $\begin{array}{l}\mathrm{EW}_{\odot} \\
(\mathrm{m} \AA)\end{array}$ \\
\hline Fe I & 5293.963 & 4.140 & -1.870 & -1.770 & $\ldots$ & $\ldots$ & -1.757 & 27.3 \\
\hline Fe I & 5379.574 & 3.690 & -1.480 & -1.510 & $\ldots$ & $\ldots$ & -1.495 & 52.1 \\
\hline Fe I & 5386.335 & 4.15 & -1.770 & -1.670 & $\ldots$ & $\ldots$ & -1.690 & 31.3 \\
\hline Fe I & 5543.937 & 4.217 & -1.140 & -1.040 & $\ldots$ & $\ldots$ & -0.980 & 52.7 \\
\hline Fe I & 5775.080 & 4.22 & -1.203 & -1.300 & $\ldots$ & -1.155 & -1.100 & 51.9 \\
\hline Fe I & 5852.217 & 4.549 & -1.330 & -1.230 & -1.170 & $\ldots$ & -1.190 & 38.1 \\
\hline Fe I & 5856.083 & 4.294 & -1.640 & -1.460 & -1.560 & $\ldots$ & -1.520 & 36.7 \\
\hline Fe I & 5859.600 & 4.550 & -0.398 & $\ldots$ & -0.610 & $\ldots$ & -0.450 & 60.1 \\
\hline Fe I & 6027.050 & 4.076 & -1.210 & -1.090 & -1.170 & $\ldots$ & -1.080 & 58.9 \\
\hline Fe I & 6078.999 & 4.652 & -1.120 & -1.020 & $\ldots$ & -1.123 & -0.930 & 46.8 \\
\hline Fe I & 6151.617 & 2.176 & -3.299 & -3.300 & -3.280 & -3.486 & -3.290 & 53.3 \\
\hline Fe I & 6165.360 & 4.143 & -1.550 & -1.460 & -1.460 & -1.645 & -1.440 & 47.7 \\
\hline Fe I & 6170.504 & 4.765 & -0.440 & -0.380 & $\ldots$ & $\ldots$ & -0.200 & 63.2 \\
\hline Fe I & 6173.341 & 2.223 & -2.880 & -2.880 & -2.880 & $\ldots$ & -2.840 & 66.9 \\
\hline Fe I & 6200.314 & 2.609 & -2.437 & -2.440 & -2.440 & $\ldots$ & -2.330 & 66.7 \\
\hline $\mathrm{Fe} \mathrm{I}$ & 6322.694 & 2.588 & -2.426 & -2.430 & -2.430 & -2.503 & -2.395 & 68.3 \\
\hline Fe I & 6481.869 & 2.279 & -2.984 & -2.980 & -2.970 & $\ldots$ & -2.940 & 65.9 \\
\hline Fe I & 6713.771 & 4.796 & -1.600 & $\ldots$ & -1.390 & -1.606 & -1.425 & 35.5 \\
\hline Fe I & 6857.243 & 4.076 & -2.150 & $\ldots$ & -2.040 & -2.203 & -2.055 & 23.4 \\
\hline Fe I & 7306.556 & 4.178 & -1.681 & $\ldots$ & $\ldots$ & -1.684 & -1.545 & 51.7 \\
\hline Fe I & 7802.473 & 5.086 & -1.584 & $\ldots$ & -1.310 & -1.493 & -1.350 & 21.2 \\
\hline $\mathrm{Fe} \mathrm{I}$ & 7807.952 & 4.990 & -0.697 & $\ldots$ & -0.510 & -0.602 & -0.477 & 66.0 \\
\hline Fe II & 5197.577 & 3.230 & -2.100 & -2.220 & $\ldots$ & $\ldots$ & -2.330 & 58.2 \\
\hline Fe II & 5234.625 & 3.221 & -2.050 & -2.180 & -2.220 & $\ldots$ & -2.285 & 57.6 \\
\hline $\mathrm{Fe}$ II & 6149.258 & 3.889 & -2.724 & $\ldots$ & -2.630 & -2.858 & -2.770 & 34.6 \\
\hline Fe II & 6247.560 & 3.892 & -2.329 & $\ldots$ & -2.270 & -2.770 & -2.385 & 47.6 \\
\hline Fe II & 6369.460 & 2.891 & -4.253 & $\ldots$ & -4.020 & $\ldots$ & -4.190 & 26.2 \\
\hline Fe II & 6456.383 & 3.903 & -2.075 & $\ldots$ & -2.060 & -2.209 & -2.145 & 56.1 \\
\hline Fe II & 6516.081 & 2.891 & -3.450 & -3.310 & $\ldots$ & $\ldots$ & -3.415 & 49.3 \\
\hline Si I & 5948.540 & 5.082 & -1.230 & -1.130 & $\ldots$ & -1.098 & -1.170 & 85.7 \\
\hline Si I & 6721.848 & 5.863 & -1.490 & $\ldots$ & -1.060 & -1.100 & -1.090 & 45.0 \\
\hline Si II & 6371.360 & 8.120 & -0.003 & $\ldots$ & -0.050 & $\ldots$ & -0.080 & 45.2 \\
\hline Si II & 6800.596 & $\ldots$ & -1.640 & $\ldots$ & $\ldots$ & $\ldots$ & -1.715 & 14.9 \\
\hline Ni I & 5805.213 & 4.168 & -0.640 & $\ldots$ & $\ldots$ & -0.530 & -0.570 & 40.5 \\
\hline Ni I & 6176.820 & 4.088 & -0.530 & $\ldots$ & -0.260 & -0.148 & -0.118 & 59.1 \\
\hline Ni I & 6378.260 & 4.154 & -0.890 & $\ldots$ & -0.830 & $\ldots$ & -0.820 & 34.4 \\
\hline Ni I & 6772.320 & 3.658 & -0.980 & $\ldots$ & -0.970 & $\ldots$ & -0.900 & 55.0 \\
\hline Ni I & 7555.598 & 3.848 & -0.046 & $\ldots$ & $\ldots$ & 0.069 & 0.059 & 85.7 \\
\hline
\end{tabular}

RAL: Ramírez et al. (2007); RTLA: Reddy et al. (2003); AMMSF: Affer et al. (2005) 
lines for the same species but corresponding to different transitions and ionization states, since this can provide useful cross-checks to test if the derived effective temperature is correct. This is particularly important for cooler stars, since it is more difficult to derive their temperature with high accuracy. We selected also some stellar lines farther in the red part of the spectrum from the linelist of Affer et al. (2005).

The first step of this procedure is to calibrate the atomic data list using the solar spectrum of Kurucz ${ }^{4}$ and the corresponding solar atmosphere, which has $T_{\text {eff }}=$ $5777 \mathrm{~K}, \log g=4.437$ and $\xi=1.5 \mathrm{~km} \mathrm{~s}^{-1}$. For each selected line we changed the oscillator strength $(\log g f)$ in the atomic linelist of Kurucz until it reproduced the observed solar spectrum. In Table 2.10 we give the original values of $\log g f$, the ones used by Ramírez et al. (2007), Reddy et al. (2003) and Affer et al. (2005), and the adopted values that we have used in our analysis. The equivalent widths of the fitted solar lines measured with the IRAF task splot are given as well. Therefore, the oscillator strengths will be fixed when fitting the spectra of the FGK stars that we have observed. After discerning which lines were suitable for the fitting procedure, we selected the value of $\log g(3.5,4.0$ or 4.5$)$ that gave the same abundances for different species and different ionization states. In this process, we also considered the proper values for the microturbulence, $\xi$, which can be expressed as a function of $T_{\text {eff }}$ and $\log g$. We used the expression of Allende Prieto et al. (2004) to derive the value of $\xi$ of these stars (see Table 2.11):

$$
\xi=1.645+3.854 \times 10^{-4} A-0.6400 B-3.427 \times 10^{-4} A \times B \mathrm{~km} \mathrm{~s}^{-1}
$$

In this expression $A=\left(T_{\text {eff }}-6387\right)$ and $B=(\log g-4.373)$.

A proper treatment of microturbulence is very important when fitting the observed lines to synthetic spectra, since the models of Kurucz are calculated assuming $\xi=2 \mathrm{~km} \mathrm{~s}^{-1}$ and the value of $[\mathrm{Fe} / \mathrm{H}]$ obtained in this way can be considerably different. After determining the metallicity considering the proper $\log g$ and $\xi$, we recalculated the $T_{\text {eff }}$ performing again the corresponding fit to synthetic photometry, which led to negligible adjustments. Another parameter that could affect the determination of metallicity is the macroturbulence. We adjusted this parameter using a rotational profile and a Gaussian broadening function independently. Both approximations led to the same final metallicities.

In Fig. 2.3 we show the spectral fits for the companions of the DA white dwarfs studied in this work. We have chosen to plot the fits corresponding to different Fe I and Fe II lines, to show how the method works for different ionization states. It is very important to adjust manually the abundances for different species and for different levels of excitation and ionization states to guarantee a good precision on the abundances derived.

\footnotetext{
${ }^{4}$ http://kurucz.harvard.edu/sun.html
} 

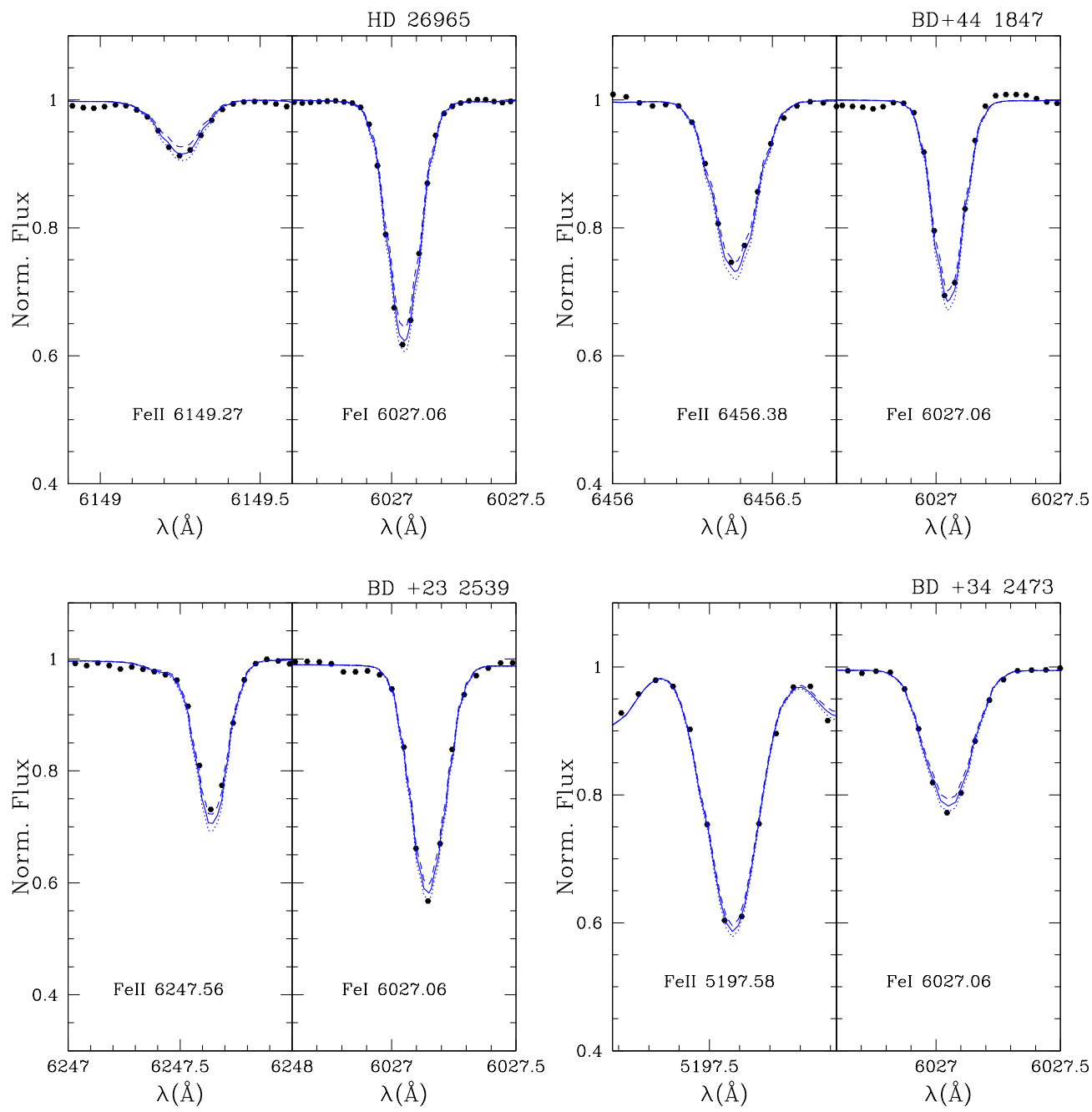

Figure 2.3: Fits of the observed spectra for some of the companions. The solid line is the fit corresponding to the derived $Z$ and the dotted and dashed lines are spectra computed for $+1 \sigma$ and $-1 \sigma$ from the average. 

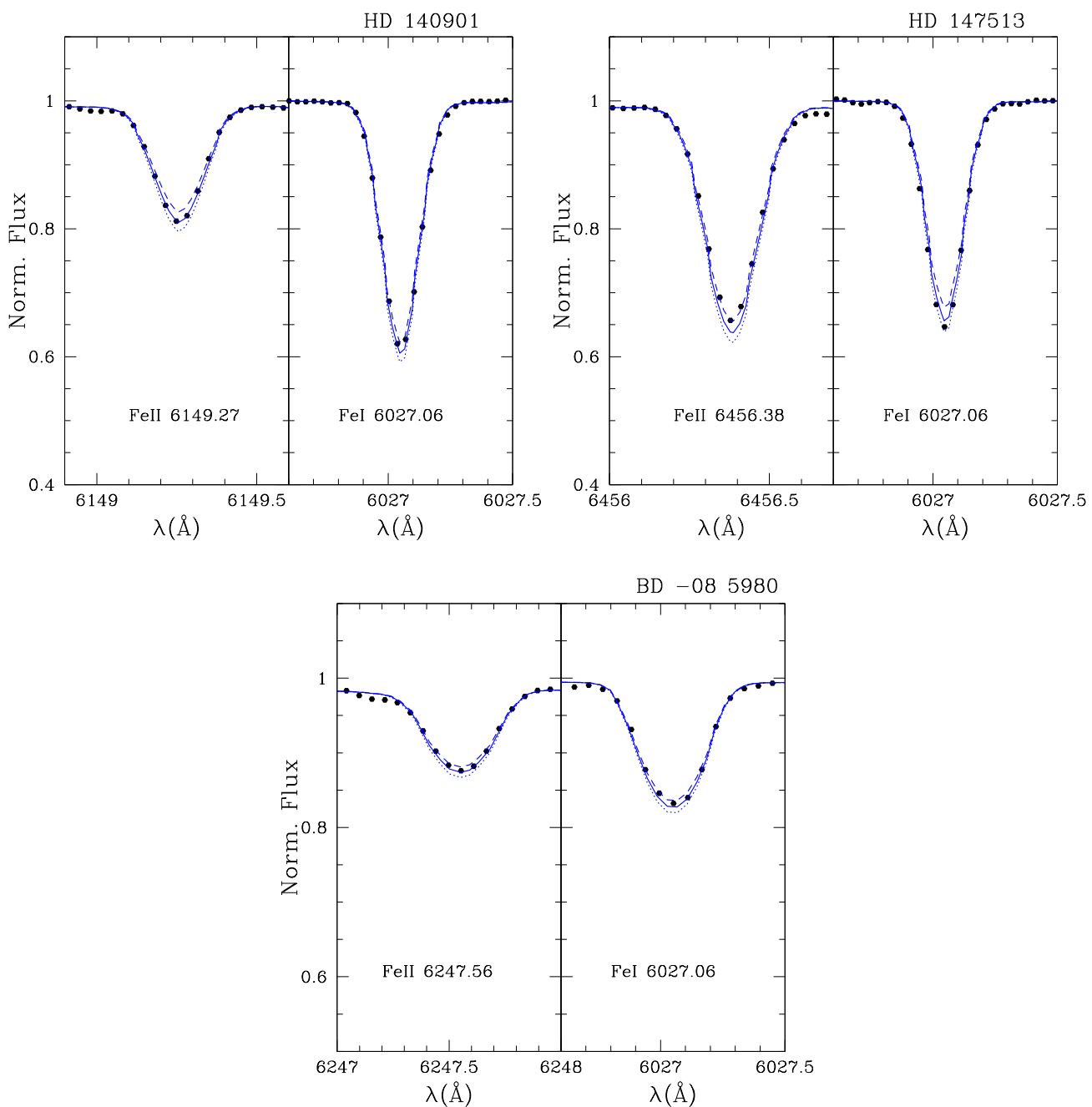

Figure 2.3: Fits of the observed spectra for some of the companions. The solid line is the fit corresponding to the derived $Z$ and the dotted and dashed lines are spectra computed for $+1 \sigma$ and $-1 \sigma$ from the average (continued). 
Table 2.11: Stellar parameters derived for the observed FGK stars.

\begin{tabular}{|c|c|c|c|c|c|c|}
\hline Name & $\begin{array}{c}\xi \\
\left(\mathrm{km} \mathrm{s}^{-1}\right)\end{array}$ & {$[\mathrm{Fe} / \mathrm{H}]$} & $Z$ & $\begin{array}{l}\log g \\
(\operatorname{dex})\end{array}$ & $\log \left(L / L_{\odot}\right)$ & $\begin{array}{l}\text { Age } \\
\text { (Gyr) }\end{array}$ \\
\hline G158- $77^{1,2}$ & $\ldots$ & $\ldots$ & $\ldots$ & $\ldots$ & $\cdots$ & $\ldots$ \\
\hline $\mathrm{BD}-01469 \mathrm{~A}^{3}$ & 0.898 & $-0.10 \pm 0.08$ & $0.016 \pm 0.003$ & 3.5 & $1.669 \pm 0.109$ & $4.17_{-2.05}^{+3.04}$ \\
\hline HD $26965^{4}$ & 1.137 & $-0.41 \pm 0.07$ & $0.008 \pm 0.001$ & 4.5 & $-0.344 \pm 0.094$ & $\ldots$ \\
\hline $\mathrm{BD}+441847^{4}$ & 1.305 & $-0.44 \pm 0.05$ & $0.007 \pm 0.001$ & 4.5 & $-0.184 \pm 0.059$ & $\ldots$ \\
\hline $\mathrm{BD}+232539^{1,4}$ & 1.317 & $-0.03 \pm 0.06$ & $0.021 \pm 0.003$ & 4.5 & $\ldots$ & $\cdots$ \\
\hline $\mathrm{BD}+342473$ & 1.524 & $-0.12 \pm 0.04$ & $0.015 \pm 0.002$ & 4.5 & $0.379 \pm 0.109$ & $3.26_{-1.46}^{+0.74}$ \\
\hline HD $140901^{4}$ & 1.306 & $0.02 \pm 0.07$ & $0.021 \pm 0.003$ & 4.5 & $-0.055 \pm 0.012$ & $\ldots$ \\
\hline HD $147513^{4}$ & 1.426 & $0.001 \pm 0.077$ & $0.020 \pm 0.003$ & 4.5 & $0.029 \pm 0.017$ & $\ldots$ \\
\hline HD $153580^{5}$ & 1.592 & $-0.01 \pm 0.01$ & $0.019 \pm 0.004$ & 4.5 & $0.671 \pm 0.042$ & $2.51_{-0.32}^{+0.34}$ \\
\hline $\mathrm{BD}-085980^{4}$ & 1.318 & $-0.37 \pm 0.05$ & $0.008 \pm 0.001$ & 4.5 & $-0.140 \pm 0.040$ & $\ldots$ \\
\hline
\end{tabular}

${ }^{1}$ The distance of this star is not known.

${ }^{2}$ Low $\mathrm{S} / \mathrm{N}$.

${ }^{3}[\mathrm{Fe} / \mathrm{H}]$ taken from McWilliam (1990).

${ }^{4}$ The total age of this star can not be determined through isochrone fitting (see text).

${ }^{5}[\mathrm{Fe} / \mathrm{H}]$ taken from Taylor (2003).

\subsubsection{Age determination}

For most stars in our sample the parallax is known (from the Hipparcos Catalogue). Thus, the calculation of the luminosity, $L$, is straightforward using the apparent magnitude after estimating the bolometric magnitude, $M_{\mathrm{bol}}$. For best accuracy we have used the $K$ band magnitude and the bolometric corrections of Masana et al. (2006), which depend on $T_{\text {eff }}$ and $[\mathrm{Fe} / \mathrm{H}]$. In Fig. 2.4 we show the HertzsprungRussell diagram for the FGK stars in our list with known distances. The isochrones of Schaller et al. (1992) for different ages and solar metallicity have been also plotted to show at which evolutionary stage are these stars located. As can be seen, the isochrone fitting technique is suitable for BD +34 2473, HD 153580 (both F stars) and for BD $-01469 \mathrm{~A}$ (K subgiant). The rest of stars are too close to the ZAMS and hence the use of isochrones does not provide accurate values for their ages. When the isochrone fitting is appropriate, we have performed an interpolation in the grid of stellar models of Schaller et al. (1992) considering the derived $T_{\text {eff }}, Z$ and $L$ to obtain the ages of these stars which yield the total ages of the white dwarfs in the common proper motion pairs. Our results are given in Table 2.11.

Another age indicator which could be used is X-ray luminosity. For some of these objects there are data available from the ROSAT All-Sky Bright Source Catalogue - 1RXS (Voges 1999) — which gives the count rate (number of detected counts per second) and the hardness ratio, $H R$. The hardness ratio is defined $H R=$ 


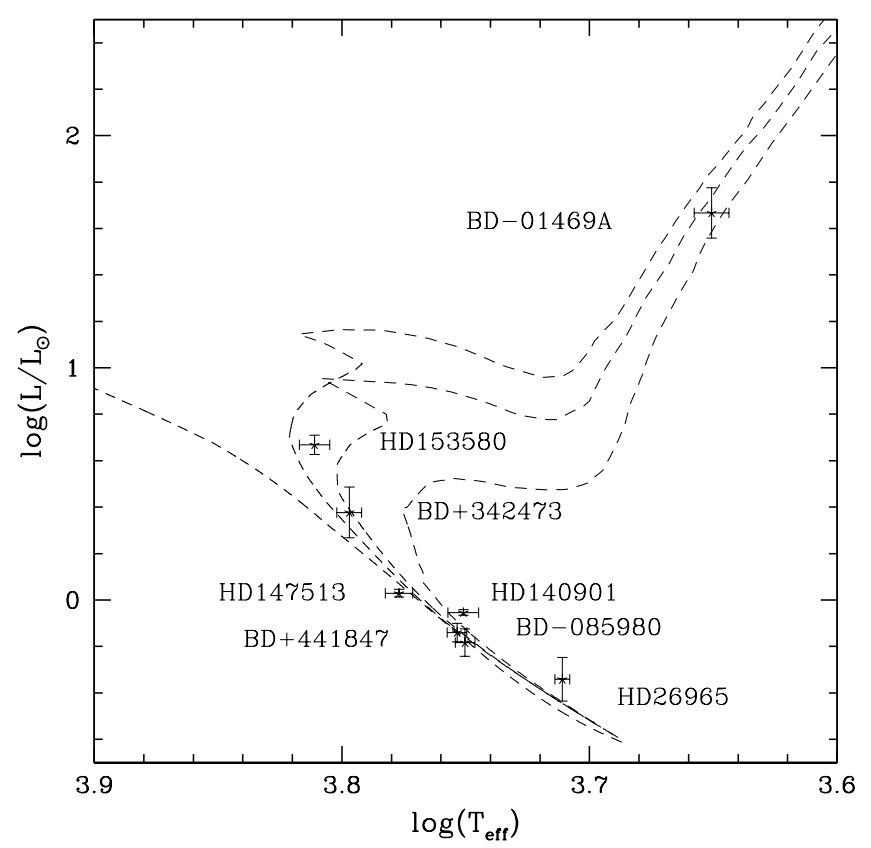

Figure 2.4: Hertzsprung-Russell diagram for the companions. The isochrones of Schaller et al. (1992) for different ages (ZAMS, 2, 3 and 7 Gyr, from left to right) and solar metallicity are also plotted.

$(H-S) /(H+S)$, where $H$ and $S$ are respectively the counts recorded in the hard and soft PSPC pulse height channels. The hard band corresponds to the count rate in PHA (Pulse Height Amplitude) channels from 52 to 201 (from $\sim 0.5$ to $2.0 \mathrm{keV}$ ) and the soft band to PHA channels from 11 to 41 ( from $\sim 0.1$ to $0.4 \mathrm{keV}$ ). To obtain the X-ray flux of a given star, we considered the calibrations of Schmitt et al. (1995). In particular, we used the conversion factor to obtain the energy flux from the measured count rate which depends on $H R$ :

$$
C F=(5.30 H R+8.31) \times 10^{-12} \mathrm{ergs} \mathrm{cm}^{-2} \text { counts }^{-1}
$$

Ribas et al. (2007) calculated a relationship between the age and X-ray luminosity for stars of different spectral types (Fig. 2.5) using both cluster data and stars belonging to wide binaries, or using kinematic criteria. In Table 2.12 we give the ROSAT information regarding these objects, the X-ray luminosity and the ages derived for the FGK companions with X-ray emission. The errors of the ages have been calculated considering the errors in the X-ray luminosity and an assumed cosmic dispersion for each relation ( 8 and $20 \%$ for $\mathrm{G}$ and $\mathrm{K}$ stars, respectively). There is also ROSAT information available for HD 153580, but since it is a member of a spectroscopic binary these relations cannot be applied. 
Table 2.12: X-ray parameters given by the ROSAT Catalog and the ages derived.

\begin{tabular}{lcccc}
\hline \hline Name & $H R$ & $\begin{array}{c}\text { Count Rate } \\
(\mathrm{c} / \mathrm{s})\end{array}$ & $\log \left(L_{x}\right)$ & $\begin{array}{c}\text { Age } \\
(\mathrm{Gyr})\end{array}$ \\
\hline HD 26965 & $-0.28 \pm 0.06$ & $0.796 \pm 0.052$ & $28.22 \pm 0.12$ & $1.07 \pm 0.37$ \\
HD 140901 & $-0.73 \pm 0.11$ & $0.150 \pm 0.023$ & $28.27 \pm 0.31$ & $0.94 \pm 0.50$ \\
HD 147513 & $-0.25 \pm 0.06$ & $0.650 \pm 0.045$ & $28.95 \pm 0.14$ & $0.33 \pm 0.12$ \\
\hline \hline
\end{tabular}

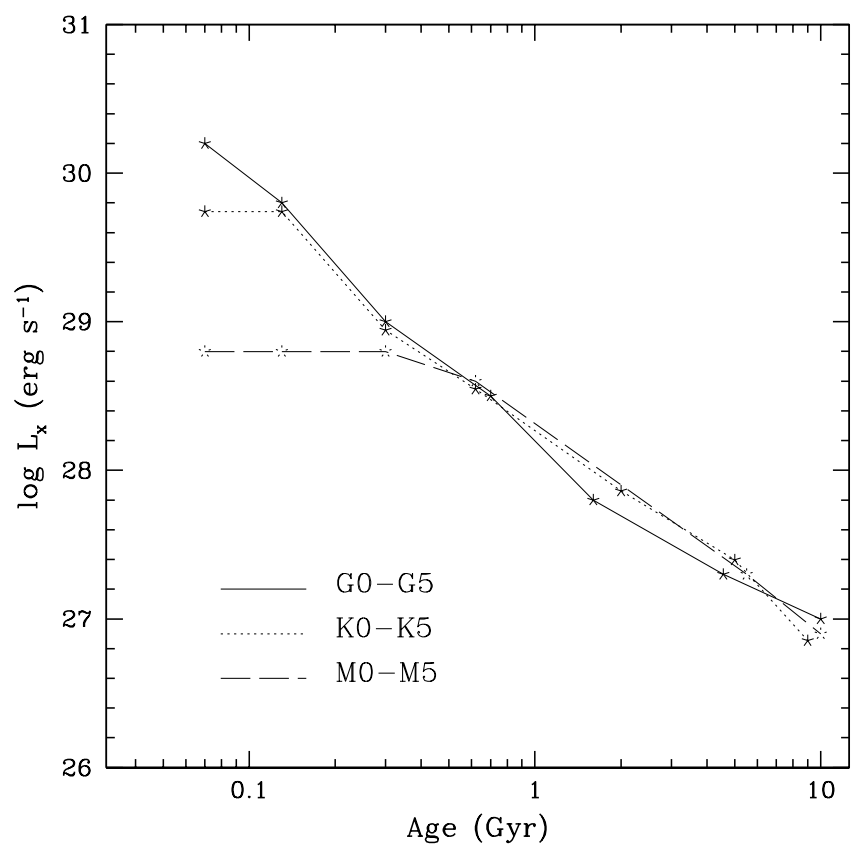

Figure 2.5: X-ray luminosity versus age for stars with different spectral types according to Ribas et al. (2007).

\subsection{Masses of the progenitors}

Once the total age of the white dwarfs and the metallicity of their progenitors are known, the initial masses can be derived interpolating suitable stellar tracks. In our case we have used the stellar tracks of Domínguez et al. (1999). The overall ages, cooling times and main-sequence lifetimes of the progenitors are given in Table 2.13. As can be noticed there are common proper motion pairs from our original list (see Table 2.1) that do not appear in this table. This is because the total age of the white dwarf member could not be derived for different reasons: either the white dwarf was 
Table 2.13: Total ages, cooling times and progenitor lifetimes for the white dwarfs in these common proper motion pairs.

\begin{tabular}{lccc}
\hline \hline Name & $\begin{array}{c}\text { Age }^{1} \\
(\mathrm{Gyr})\end{array}$ & $\begin{array}{c}t_{\text {cool }} \\
(\mathrm{Gyr})\end{array}$ & $\begin{array}{c}t_{\text {prog }} \\
(\mathrm{Gyr})\end{array}$ \\
\hline WD0315-011 & $4.17_{-2.05}^{+3.04}$ & $1.20 \pm 0.56$ & $2.97_{-2.12}^{+3.09}$ \\
WD0413-017 & $1.07 \pm 0.37$ & $0.112 \pm 0.008$ & $0.96 \pm 0.37$ \\
WD1354+340 & $3.26_{-1.46}^{+0.74}$ & $0.20 \pm 0.02$ & $3.06_{-1.46}^{+0.74}$ \\
WD1544-377 & $0.94 \pm 0.50$ & $0.76 \pm 0.05$ & $0.18 \pm 0.50$ \\
WD1620-391 & $0.33 \pm 0.12$ & $0.026 \pm 0.001$ & $0.30 \pm 0.12$ \\
WD1659-531 & $2.51_{-0.32}^{+0.34}$ & $0.24 \pm 0.01$ & $2.27_{-0.32}^{+0.34}$ \\
\hline \hline
\end{tabular}

${ }^{1}$ For the rest of common proper motion pairs the total age of the white dwarf could not be derived (see text).

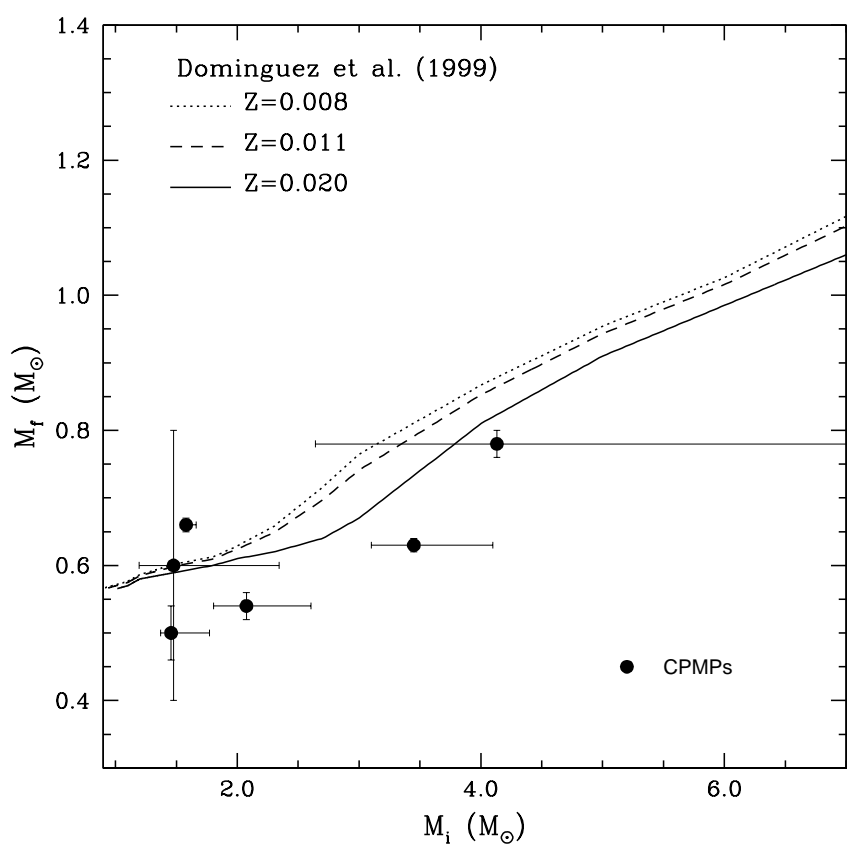

Figure 2.6: Final masses versus initial masses for the common proper motion pairs (CPMPs) studied here. 
Table 2.14: Initial and final masses, and metallicities for the white dwarfs in these common proper motion pairs.

\begin{tabular}{lccc}
\hline \hline Name & $\begin{array}{c}M_{\mathrm{f}} \\
\left(M_{\odot}\right)\end{array}$ & $\begin{array}{c}M_{\mathrm{i}} \\
\left(M_{\odot}\right)\end{array}$ & $Z$ \\
\hline WD0315-011 & $0.60 \pm 0.20$ & $1.48_{-0.28}^{+0.87}$ & $0.016 \pm 0.003$ \\
WD0413-017 & $0.54 \pm 0.02$ & $2.07_{-0.27}^{+0.53}$ & $0.008 \pm 0.001$ \\
WD1354+340 & $0.50 \pm 0.04$ & $1.46_{-0.09}^{+0.31}$ & $0.015 \pm 0.002$ \\
WD1544-377 & $0.78 \pm 0.02$ & $4.13_{-1.49}^{+?}$ & $0.021 \pm 0.003$ \\
WD1620-391 & $0.63 \pm 0.01$ & $3.45_{-0.35}^{+0.65}$ & $0.020 \pm 0.003$ \\
WD1659-531 & $0.66 \pm 0.01$ & $1.58_{-0.05}^{+0.08}$ & $0.019 \pm 0.004$ \\
\hline \hline
\end{tabular}

not of DA spectral type, the distance of the pair was not known, the companion was not evolved enough to use stellar isochrones to derive its age through isochrone fitting or the companion did not present $\mathrm{X}$-ray emission or at least these data was not in the ROSAT database. From Table 2.13 it can be noted all the total ages exceed the cooling times, as expected. The initial and final masses obtained are detailed in Table 2.14. In Fig. 2.6 we represent the final masses versus the initial masses obtained for the white dwarfs in the common proper motion pairs from which the age and metallicity have been derived. We also plot the theoretical initial-final mass relationship of Domínguez et al. (1999) for different metallicities.

From an inspection of Fig. 2.6 it can be noted that our results present a considerable dispersion, which is larger than the uncertainties, in comparison with the theoretical initial-final mass relationships of Domínguez et al. (1999). According to our results a main-sequence star of $1.5 M_{\odot}$ with solar metallicity could end up as a white dwarf with masses that differ by $\sim 25 \%$ (cf. WD1354+340 and WD1659-531). Moreover, two white dwarfs of nearly the same masses could come from main-sequence stars with masses different by a factor of 2 (cf. WD1620-391 and WD1659-531). Apparently, this difference is not a consequence of metallicity, since its practically the same for these objects (see Table 2.14). However, it is also interesting to note that the influence of metallicity in the theoretical initial-final mass relationship seems to be almost negligible below $2 M_{\odot}$. Other factors, such as magnetic fields or rotation (Domínguez et al. 1996) should be studied in detail in order to discern their effect on this relation. A thorough discussion of our results can be found in chapter 4 . 



\section{Chapter 3}

\section{The peculiar case of WD0433+270}

\subsection{Introduction}

Among the common proper motion pairs that we have studied in the previous chapter with the purpose of improving the initial-final mass relationship G39-27/289 has turned out to be very peculiar. This is the reason why we discuss it in a separate chapter. This common proper motion pair is comprised of a DA white dwarf (WD0433+270) and a K type star (BD +26 730) (Holberg et al. 2002), which is a well studied variable star (V833 Tau) and also a single-lined spectroscopic binary with a very low-mass companion (Tokovinin et al. 2006). As in the case of the rest of common proper motion pairs of our sample we can assume that they were born simultaneously and with the same chemical composition (Wegner 1973; Oswalt et al. 1988). Since the components are well separated ( $\sim 2200$ AU in this case), no mass exchange has taken place and we can consider that they have evolved as isolated stars. Thus, it is logical to assume that both components of G39-27/289 have the same age and the same original metallicity. The K-star component, and, by extension, its common proper motion companion, were classified as Hyades members by Perryman et al. (1998) and therefore would have an age ranging from about 0.6 to 0.7 Gyr. However, this stands in obvious conflict with the cooling time of WD0433+270, which has been estimated to be about 4 Gyr (Bergeron et al. 2001).

\subsection{Properties of the pair}

As explained in the previous chapter, in the case of $\mathrm{BD}+26730$, we performed spectroscopic observations with the FOCES echelle spectrograph on the $2.2 \mathrm{~m}$ telescope at CAHA (Almería, Spain), obtaining a resolution of $R \sim 47000$. From these observations, together with a detailed study of the visible and near-infrared spectral 
Table 3.1: Stellar parameters derived for BD+26 730 .

\begin{tabular}{lccc}
\hline \hline$T_{\text {eff }}(\mathrm{K})$ & {$[\mathrm{Fe} / \mathrm{H}]$} & $Z$ & $\log \left(L / L_{\odot}\right)$ \\
\hline $4595 \pm 30$ & $0.03 \pm 0.09$ & $0.021 \pm 0.004$ & $-0.527 \pm 0.021$ \\
\hline \hline
\end{tabular}

energy distribution of the star, we derived the effective temperature and metallicity of $\mathrm{BD}+26730$ following the procedure described in $\S 2.4$. The resulting value of $T_{\text {eff }}=4595 \pm 30 \mathrm{~K}$ is in good agreement with the determination of Oláh et al. (2001). The chemical composition analysis yielded a nearly solar metallicity of $[\mathrm{Fe} / \mathrm{H}]=0.03 \pm 0.09$. The total luminosity, $L$, was calculated from the apparent magnitude and a bolometric correction (Masana et al. 2006). A summary of the derived stellar parameters is given in Table 3.1.

A very relevant parameter for our study is the age of BD+26 730. Unfortunately, this cannot be determined reliably from the observed $T_{\text {eff }}$ and $L$ and using model isochrones because of the relative proximity of the star to the ZAMS. Note, however, that Barrado y Navascués \& Stauffer (1996) determined lithium abundances for a sample of binaries classified as Hyades members, including BD+26 730 (J301). Lithium was indeed detected in this case, obtaining a value of $\log N_{\mathrm{Li}}=0.31$, which is in good accord with the rest of the observed binaries, albeit with an abundance somewhat larger than that of single objects belonging to the Hyades $\left(\log N_{\mathrm{Li}}=-0.5\right)$. According to Barrado y Navascués \& Stauffer (1996), this is something expected since, in general, binary systems have an overabundance of lithium with respect to single stars. Later, Barrado y Navascués et al. (1997) recalculated the lithium abundance of $\mathrm{BD}+26730$, obtaining in this case $\log N_{\mathrm{Li}}=0.23$. They compared the $\mathrm{Li}$ abundance and the effective temperature of this object with lithium depletion isochrones, yielding an age of $\sim 600 \mathrm{Myr}$. Thus, the results suggest a Hyades age for $\mathrm{BD}+26730$.

In the case of WD0433+270, a cool white dwarf, the best way to estimate its effective temperature is by using the photometric energy distribution instead of a spectroscopic fit (Bergeron et al. 2001). However, the observations were also useful to ensure its spectral classification. As explained in $\S 2.2$, we performed observations with the LCS spectrograph of the Harlan J. Smith $(2.7 \mathrm{~m})$ telescope at McDonald Observatory (Texas, USA) covering some of the main Balmer lines (from 3885 to $5267 \AA$ ) and obtaining a resolving power of $\sim 5 \AA$ FWHM. The spectrum obtained shows weak absorption lines but we were able to unambiguously identify $\mathrm{H}_{\beta}$. The weakness of the lines is the reason why this star had been previously classified as a type DC white dwarf by several authors (Eggen \& Greenstein 1965; Oswalt \& Strunk 1994). Higher resolution observations by Bergeron et al. (2001) and Zuckerman et al. (2003) had permitted the identification of $\mathrm{H}_{\alpha}$ and $\mathrm{H}_{\beta}$, respectively. Putting all results 
Table 3.2: Photometric properties and parallax of WD0433+270. The uncertainties are typically $3 \%$ at $\mathrm{V}, \mathrm{R}$, and $\mathrm{I}$, and $5 \%$ in the rest.

\begin{tabular}{lccccccc}
\hline \hline$B$ & $V$ & $R$ & $I$ & $J$ & $H$ & $K$ & $\pi$ (mas) \\
\hline 16.48 & 15.81 & 15.40 & 15.01 & 14.61 & 14.42 & 14.32 & $60 \pm 3$ \\
\hline \hline
\end{tabular}

Table 3.3: Atmospheric parameters of WD0433+270 available in the literature.

\begin{tabular}{lcc}
\hline \hline$T_{\text {eff }}(\mathrm{K})$ & $\log g($ dex $)$ & Reference \\
\hline $5620 \pm 110$ & $8.14 \pm 0.07$ & Bergeron et al. (2001) \\
$5434 \pm 300$ & $8.0 \pm 0.1$ & Zuckerman et al. (2003) \\
\hline \hline
\end{tabular}

together we can confidently classify this object as a DA white dwarf.

Bergeron et al. (2001) used BVRIJHK photometry - see Table 3.2) — to obtain the energy flux distribution of WD0433+270. From a fit of different theoretical energy distributions they obtained its effective temperature and $(R / d)^{2}$, where $R$ is the radius of the star and $d$ is the distance. The radius can be determined accurately because a trigonometric distance is available for this object. The authors also obtained its mass and $\log g$ from the mass-radius relation of Fontaine et al. (2001) for C/O white dwarfs. Zuckerman et al. (2003) followed the same procedure as Bergeron et al. (2001), but using the mass-radius relation of Wood (1995). The results obtained by these authors are summarized in Table 3.3. It is worth mentioning that the spectroscopic observations were not used in any case to estimate the stellar properties but they served as an internal check of the photometric solutions via comparison of calculated models with the observed line profiles.

The luminosity of the white dwarf can be derived from its effective temperature (Bergeron et al. 2001) and distance $(d=16.95 \pm 0.86 \mathrm{pc})$, and considering its apparent magnitude and the bolometric corrections of Holberg \& Bergeron (2006). The value that we obtained is $\log \left(L / L_{\odot}\right)=-3.92 \pm 0.04$, and, from this, a radius of $R=$ $0.0115 \pm 0.0010 R_{\odot}$. Both results are in good agreement with those reported by Bergeron et al. (2001).

\subsection{Membership to the Hyades}

The membership of WD0433+270 to the Hyades cluster was evaluated by Eggen (1993a) within the course of a study of all degenerate stars with certain kinematic criteria. WD0433+270 was excluded from the member list, although the radial velocity of its companion is identical to the value predicted from membership. The 
Table 3.4: Spatial velocities for the studied stars and the Hyades cluster and streams.

\begin{tabular}{lcccccc}
\hline \hline & $\begin{array}{c}\mu_{\alpha} \\
(\mathrm{mas} / \mathrm{yr})\end{array}$ & $\begin{array}{c}\mu_{\delta} \\
(\mathrm{mas} / \mathrm{yr})\end{array}$ & $\begin{array}{c}V_{r} \\
(\mathrm{~km} / \mathrm{s})\end{array}$ & $\begin{array}{c}U \pm \sigma_{U} \\
(\mathrm{~km} / \mathrm{s})\end{array}$ & $\begin{array}{c}V \pm \sigma_{V} \\
(\mathrm{~km} / \mathrm{s})\end{array}$ & $\begin{array}{c}W \pm \sigma_{W} \\
(\mathrm{~km} / \mathrm{s})\end{array}$ \\
\hline WD0433+270 & 228 & -155 & +36.3 & -39.2 & -15.5 & -1.8 \\
WD0433+270 & 228 & -155 & +41.7 & -44.4 & -15.7 & -3.9 \\
$\mathrm{BD}+26730^{3}$ & 232.36 & -147.11 & $+36.18 \pm 0.08$ & -39.4 & -17.2 & -1.6 \\
Hyades $\mathrm{OCl}^{4}$ & $\ldots$ & $\ldots$ & $\ldots$ & $-42.8 \pm 3.6$ & $-17.9 \pm 3.2$ & $-2.2 \pm 5.2$ \\
Hyades SCl$(1)^{4}$ & $\ldots$ & $\ldots$ & $\ldots$ & $-31.6 \pm 2.8$ & $-15.8 \pm 2.8$ & $0.8 \pm 2.7$ \\
Hyades SCl$(2)^{4}$ & $\ldots$ & $\ldots$ & $\ldots$ & $-33.0 \pm 4.2$ & $-14.1 \pm 4.0$ & $-5.1 \pm 3.1$ \\
Hyades SCl$(3)^{4}$ & $\ldots$ & $\ldots$ & $\ldots$ & $-32.8 \pm 2.8$ & $-11.8 \pm 2.8$ & $-8.9 \pm 2.9$ \\
Hyades Stream $^{5}$ & $\ldots$ & $\ldots$ & $\ldots$ & $-30.3 \pm 1.5$ & $-20.3 \pm 0.6$ & $-8.8 \pm 4.0$ \\
\hline \hline
\end{tabular}

${ }^{1}$ Proper motions from Salim \& Gould (2003) and velocities from Zuckerman et al. (2003).

${ }^{2}$ Proper motions from Salim \& Gould (2003) and velocities recalculated considering a Fe-core composition (see text).

${ }^{3}$ Spatial velocities calculated from the radial velocity and proper motions reported by Perryman et al. (1997).

${ }^{4}$ Spatial velocities from Chereul et al. (1999).

${ }^{5}$ Spatial velocities from Famaey et al. (2005).

justification was a color index supposedly too red to belong to the Hyades but, more importantly, the distance of the common proper motion pair to the center of the cluster. This author concluded that this common proper motion pair is projected on the cluster but only at about a third of the distance of the cluster.

The comprehensive study of Perryman et al. (1998) considered the 5490 Hipparcos Catalog stars corresponding to the field of the Hyades. Hipparcos astrometry was combined with radial velocity measurements in order to obtain three-dimensional velocities, which allowed candidate membership selection based on position and kinematic criteria. The authors divided the Hyades into four components by using the three-dimensional distance to the cluster center. The distance of BD +26730 to the center of the cluster is $29 \mathrm{pc}$ and therefore it was classified as a former member of the Hyades cluster, currently lying beyond the tidal radius ( $\sim 10 \mathrm{pc})$. In contrast, de Bruijne (1999) preferred not to include BD +26 730 in their list of Hyades member stars following the conclusions of a study based on the convergent-point method.

It is also worth mentioning two further pieces of evidence that were not included in the studies mentioned above. One is the metal content of $\mathrm{BD}+26730$. As discussed previously, we have reliably determined the metallicity of this star and obtained a value of $[\mathrm{Fe} / \mathrm{H}]=0.03 \pm 0.09$. This is in reasonably good agreement with the Hyades metallicity of $[\mathrm{Fe} / \mathrm{H}]=0.14 \pm 0.05$ as determined by Perryman et al. (1998). The result is not conclusive, however, because the mean metallicity of field 
stars is of $[\mathrm{Fe} / \mathrm{H}]=-0.14 \pm 0.19$ (Nordström et al. 2004). The other important point is the detection of lithium in the spectrum of $\mathrm{BD}+26730$ by Barrado y Navascués \& Stauffer (1996), which clearly favors a relatively young age for this object, and in agreement with the rest of the Hyades members studied.

It has been often mentioned that there is a spatially unbound group of stars in the solar neighborhood with the same kinematics as the Hyades open cluster (Eggen 1993b; Perryman et al. 1998). This group of stars is called the Hyades stream or Hyades supercluster. Chereul et al. (1999) mapped the density-velocity inhomogeneities of an absolute magnitude limited sample of $\mathrm{A}-\mathrm{F}$ type dwarfs. Three different clumps within the Hyades stream were distinguished, each one of them with characteristic space velocities, which are given in Table 3.4. The authors also claimed that the Hyades stream contains probably three groups of 0.5-0.6 Gyr, 1 Gyr and 1.6-2 Gyr, which are in an advanced stage of dispersion in the same velocity volume. Each stream presents a characteristic age distribution, although the velocity separation does not produce a clear age separation.

In Table 3.4 we provide the radial and space velocities of $\mathrm{BD}+26730$ and WD0433+270. We also give the kinematic properties of the Hyades open cluster (OCl) and of each clump within the stream according to Chereul et al. (1999) called SCl 1, 2 and 3 by these authors. The recent spatial velocities of the Hyades stream calculated by Famaey et al. (2005) are also listed. As can be seen, both members of the common proper motion pair have velocities compatible with those of the Hyades open cluster and are somewhat different from the velocities characteristic of the Hyades stream or the clumps within it.

From the kinematic considerations made here, together with the lithium detection in $\mathrm{BD}+26730$, we favor the hypothesis that the common proper motion pair studied here is indeed linked with the Hyades cluster evolution-wise. It is certainly not a bona fide cluster member because of it location beyond the tidal radius of the cluster but it is likely a former member that has escaped. If this scenario is correct, the components of the pair should have the age of the Hyades cluster, which was estimated to be $625 \pm 50 \mathrm{Myr}$ by Perryman et al. (1998). However, we do not have conclusive evidence supporting this evolutionary link and therefore the alternative scenario that this pair belongs to the Hyades stream cannot be completely ruled out.

\subsection{The nature of WD0433+270}

Most white dwarfs have a core made of carbon and oxygen, although other compositions are possible. However, in this particular case, and given the putative age discrepancy with the Hyades cluster, we consider all the feasible compositions that have been proposed to date. In Fig. 3.1 we show mass-radius relations considering different internal compositions: He core (Serenelli et al. 2002), C/O core (Salaris et al. 2000), O/Ne core (Althaus et al. 2005) and Fe core (Panei et al. 2000a). All 


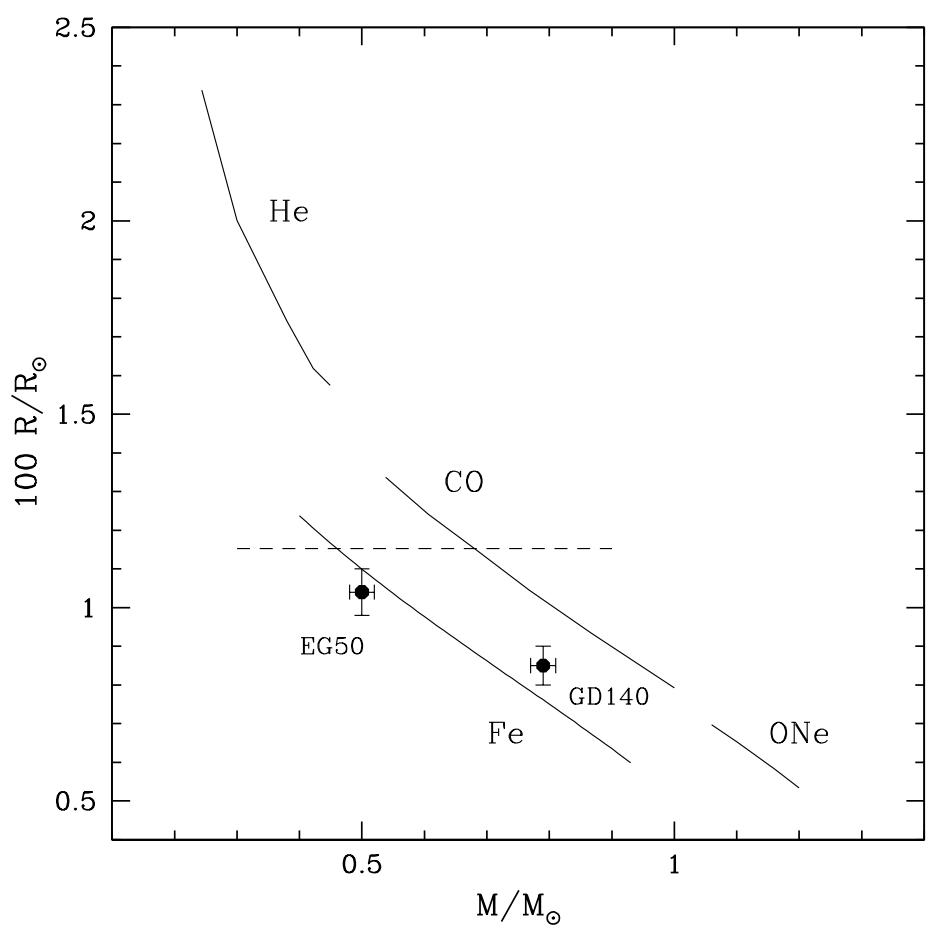

Figure 3.1: Mass-radius relations for different compositions and $T_{\text {eff }}=5500 \mathrm{~K}$. The dashed line corresponds to the radius of WD0433+270. The filled circles correspond to observational data obtained by Provencal et al. (1998).

these relations correspond to an effective temperature of $5500 \mathrm{~K}$. As can be seen, the radius of WD0433+270 only has a corresponding mass in the case of $\mathrm{C} / \mathrm{O}$ and Fe cores (dashed line). The existence of iron white dwarfs has been pointed out in the past. Provencal et al. (1998) derived the radii of some field white dwarfs using parallaxes and effective temperatures. Then, they obtained the masses from the radii and surface gravities, which were previously determined independently from spectroscopic fits to white dwarf models. In two cases (GD140 and EG50) they obtained masses that were too small to fit the $\mathrm{C} / \mathrm{O}$ mass-radius relation. The best explanation for these stars that the authors proposed is that they have a Fe core (Shipman \& Provencal 1999). The data corresponding to these stars have been also plotted in Fig. 3.1.

The gravitational redshift and the radial velocity of a white dwarf are two interdependent parameters. Zuckerman et al. (2003) calculated the radial velocity of WD0433+270 (see Table 3.4) assuming a C/O composition. Since the Fe composition seems also possible in this case, we have recalculated this value considering the 
Table 3.5: Masses and cooling times for WD0433+270 assuming a C/O and Fe core.

\begin{tabular}{ccc}
\hline \hline Model & $M_{\mathrm{WD}}\left(M_{\odot}\right)$ & $t_{\text {cool }}(\mathrm{Gyr})$ \\
\hline $\mathrm{C} / \mathrm{O}$ core $^{1}$ & $0.67 \pm 0.03$ & $4.1 \pm 1.2$ \\
Fe core $^{2}$ & $0.46 \pm 0.07$ & $1.0 \pm 0.1$ \\
\hline \hline
\end{tabular}

${ }^{1}$ Salaris et al. (2000).

${ }^{2}$ Panei et al. (2000a)

mass-radius relation of Panei et al. (2000a) for Fe white dwarfs. As seen in Table 3.4 , in both cases, the velocities are compatible with the kinematic properties of the Hyades cluster, considering the typical errors of white dwarf radial velocities (Schultz et al. 1996).

Turning the argument around, the kinematics can also be used to estimate the mass of WD0433+270 independently of the composition of the core. This can be done assuming that WD0433+270 has the same radial velocity as its companion, $\mathrm{BD}+26$ 730, and considering the observed velocity of this white dwarf reported by Zuckerman et al. (2003). This yields a mass of $\sim 0.55 M_{\odot}$, which does not conclusively favor any of the two compositions $(\mathrm{C} / \mathrm{O}$ or $\mathrm{Fe})$.

Once we know the masses that correspond to each mass-radius relation (see Table 3.5) we can calculate the cooling times using the proper cooling sequences. In Fig. 3.2 we show the cooling sequences: $\mathrm{C} / \mathrm{O}$ core (top-left), O/Ne core (topright), He core (bottom-left) and Fe core (bottom-right). Dashed lines correspond to cooling isochrones. We overplotted the effective temperature and luminosity of WD0433+270 to check whether it falls within the range of values corresponding to each set of cooling sequences. As expected, there is no correspondence with the model sequences with $\mathrm{O} / \mathrm{Ne}$ and $\mathrm{He}$ cores since the physics of white dwarfs with these compositions predicts masses for this radius that are too high or too low, respectively.

The most common internal structure of a white dwarf is thought to be a core made of $\mathrm{C} / \mathrm{O}$ surrounded by a $\mathrm{H}$ thick envelope on top of a He buffer, with compositions of $q(\mathrm{H})=M_{\mathrm{H}} / M=10^{-4}$ and $q(\mathrm{He})=M_{\mathrm{He}} / M=10^{-2}$. The cooling sequences of Salaris et al. (2000) consider such configuration, but with a higher abundance of $\mathrm{O}$ than $\mathrm{C}$ at the center of the core. These improved cooling sequences include an accurate treatment of the crystallization process of the $\mathrm{C} / \mathrm{O}$ core, including phase separation upon crystallization, together with state-of-the-art input physics suitable for computing white dwarf evolution. As can be seen in Table 3.5, the cooling time derived ( $\sim 4 \mathrm{Gyr})$ is in excellent agreement with the results obtained by Bergeron et al. (2001). This cooling time is 6 times larger than the age of the Hyades open cluster and 2 times larger than that of the older Hyades stream group ( 2 Gyr, 

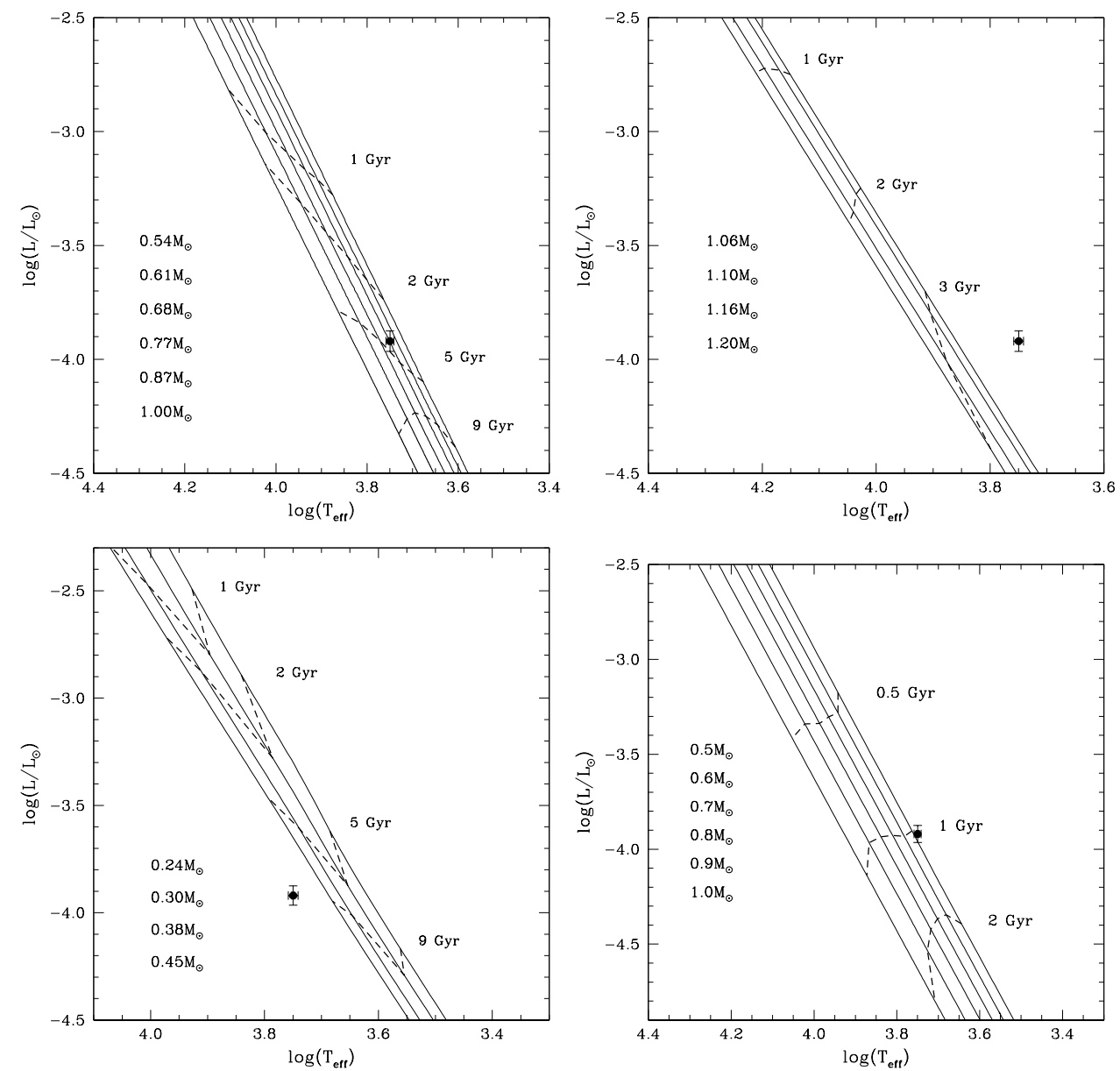

Figure 3.2: Luminosity versus effective temperature for different cooling tracks: $\mathrm{C} / \mathrm{O}$ core (top-left), O/Ne core (top-right), He core (bottom-left) and Fe core (bottom-right). Dashed lines represent cooling isochrones.

Chereul et al. 1999). The assumption of a O/Ne-core white dwarf will give smaller cooling times, since the heat capacity of $\mathrm{O}$ and $\mathrm{Ne}$ is lower than the one of $\mathrm{C}$, and as result white dwarfs of this type cool faster (by a factor of 2). We considered the cooling tracks of Althaus et al. (2007) for white dwarfs with O/Ne cores (Fig. 3.2, topright). The outer layer chemical stratification consists of a pure hydrogen envelope of $10^{-6} M_{\odot}$ overlying a helium-dominated shell of $4 \times 10^{-4} M_{\odot}$ and, below that, a buffer rich in ${ }^{12} \mathrm{C}$ and ${ }^{16} \mathrm{O}$. However, as mentioned before, this core composition does not reproduce a white dwarf with such small radius.

In light of the recent results obtained by Kilic et al. (2007), who claim that He 
white dwarfs may exist in the Galaxy as a product of single evolution, we have considered also this composition. In Fig. 3.2 (bottom-left) we have plotted the cooling sequences of Serenelli et al. (2002) for He-core white dwarfs. But, as in the case of the models with $\mathrm{O} / \mathrm{Ne}$ cores they do not reproduce a white dwarf with this observed radius. Moreover, as can be seen in Fig. 3.2, the effective temperature and luminosity of WD0433+270 does not fall in the range of values covered by these tracks. In any case, He-core white dwarfs cool typically slower than $\mathrm{C} / \mathrm{O}$-core white dwarfs because of the higher heat capacity of $\mathrm{He}$ in comparison with $\mathrm{C}$ and $\mathrm{O}$. Although this is partly compensated by the smaller mass, the net result does not lead to smaller cooling times.

Finally, we have taken into account the cooling tracks of Panei et al. (2000b) for Fe-core white dwarfs, obtaining a cooling time of $\sim 1$ Gyr, which is 4 times smaller than for $\mathrm{C} / \mathrm{O}$ white dwarfs. This is because iron nuclei are much heavier than $\mathrm{C}$ or $\mathrm{O}$ and, hence, the specific heat per gram is much smaller.

The cooling time derived is in much better agreement with the age of the Hyades open cluster when considering an Fe core. It should be noted that the cooling time of a white dwarf is just a lower limit to its total age, since the main-sequence lifetime of its progenitor, which is badly determined for single white dwarfs, should also be taken into account. However, this value could be relatively small if the white dwarf progenitor is massive ( $\left.t_{\text {prog }} \ll 1 \mathrm{Gyr}\right)$. It is worth mentioning that the uncertainties in the cooling times are derived only from the observational parameters and do not consider a possible systematic contribution from the cooling sequences.

\subsection{Discussion}

Considering the kinematic properties of the members of the pair and the lithium detection in $\mathrm{BD}+26730$, we favor the scenario in which the common proper motion pair is indeed a former member of the Hyades cluster, and thus its members have a coeval age of $\sim 0.6-0.7$ Gyr. Having evaluated different compositions for WD0433+270, the young age inferred from cluster membership is only compatible with the case of a Fe core, which would have an associated cooling time of $\sim 1 \mathrm{Gyr}$. The agreement is not perfect, but the modelling of the cooling evolution of Fe-core white dwarfs could still have some associated uncertainties.

The existence of white dwarfs with an Fe-rich core has important consequences for the models of thermonuclear explosion of stellar degenerate cores. According to the theory of stellar evolution, all stars that develop an Fe core experience a collapse to a neutron star or a black hole, regardless of the mass loss rate assumed. However, there is still an alternative possibility to avoid this final fate that relies in the failure of the thermonuclear explosion of a degenerate white dwarf near the Chandrasekhar limit (Isern et al. 1991). Our current view of a thermonuclear explosion is as follows. Once the thermonuclear runaway starts in the central regions of a white dwarf, the 
ignition front propagates outwards and injects energy at a rate that depends on the velocity at which matter is effectively burned leading to the expansion of the star. At the same time, electron captures on the incinerated matter efficiently remove energy at a rate determined by the density causing the contraction of the star. Therefore, depending on the ignition density and the velocity of the burning front, the outcome can be different. It is known that He-degenerate cores always explode, that $\mathrm{O} / \mathrm{Ne}$ and $\mathrm{Fe}$ degenerate cores always collapse and that $\mathrm{C} / \mathrm{O}$-degenerate cores can explode or collapse depending on the ignition density. The existence of Fe-rich white dwarfs would imply the possibility of an intermediate behavior between those discussed above in which an Fe remnant is left after a mild explosion.

A detailed theory explaining the formation of Fe-core white dwarfs is still to be developed and up to now the possibility of their existence has been suggested mainly from observational evidences. Other examples of possible Fe-core white dwarfs have been found in the past. Provencal et al. (1998) singled out two objects whose radii and masses were too small to fit the typical $\mathrm{C} / \mathrm{O}$ mass-radius relation. These authors analyzed a sample of white dwarfs, calculated their masses using either orbital parameters, gravitational redshifts or spectroscopic fits, and determined their radii independently from the knowledge of effective temperatures and distances. Provencal et al. (1998) indicated that in those two cases, a core made by Fe was the only plausible explanation that could account for their small radii. It is worth to mention that among these presumably Fe white dwarfs that Provencal et al. (1998) found there was also Procyon B. In this case, a bad estimation of its effective temperature, which was initially higher, led the authors to derive a smaller radius, which shifted the location of Procyon B in the mass-radius panel to a region more compatible with Fe-core white dwarfs. According to the new estimation of the effective temperature, the most compatible composition for Procyon B is a pure C-core surrounded by Herich envelope, with also some traces of $\mathrm{C}, \mathrm{Mg}, \mathrm{Ca}$ and $\mathrm{Fe}$, which makes this star quite peculiar (Provencal et al. 2002). It is therefore very important to use accurate values of effective temperatures and masses to reach definitive conclusions about the compositions of white dwarfs. Thus, a more detailed and precise photometric and kinematic study of WD0433+270 will help to confirm or reject the Fe-core hypothesis.

Besides the Fe-core hypothesis, there is an alternative scenario that we cannot rule out. Although we think it is unlikely, the common proper motion pair might not be related in any way to the Hyades cluster. This would eliminate the age constraint and thus relax the requirement of an exotic composition for the white dwarf core. In this case, the age of the objects (both WD0433+270 and BD+26 730) could be of $\sim 4$ Gyr. This would have some interesting consequences to our current view of star streams or moving groups in the Galaxy. As pointed out by Chereul et al. (1999), the Hyades stream contains at least three distinct age groups with ages up to 2 Gyr. The upper limit was probably a consequence of the use of $\mathrm{A}-\mathrm{F}$ stars as kinematic tracers, which would naturally have an age cutoff at about 2-3 Gyr. With an age of 
4 Gyr, WD0433+270 and BD+26 730 would indicate the existence of an even older population in the Hyades stream thus completely ruling out any coevality within the members. This would lend strong support to the model of a resonant origin for the Hyades stream (Famaey et al. 2007). Another implication of such an old age is the conflict with lithium detection, which would imply much lower destruction rate than expected.

Definitive proof supporting one of these scenarios will have to await an unambiguous determination of the mass of this object or, alternatively, a more conclusive study on its evolutionary link with the Hyades cluster. 



\section{Chapter 4}

\section{Revision of the current initial-final mass relationship}

\subsection{Introduction}

Up to now, most part of the effort to improve the initial-final mass relationship from the observational point of view has been focused in observing white dwarfs in open clusters (Ferrario et al. 2005; Dobbie et al. 2006). This approach consists in performing spectroscopic observations of white dwarfs belonging to a cluster and deriving their atmospheric parameters (effective temperature and surface gravity) from a fit of their spectra to synthetic models. Their masses and cooling times are then obtained using the proper cooling tracks. The metallicity and age of the cluster is independently estimated from spectroscopic observations of less evolved stars and from the fit of the main-sequence turn-off of the cluster with isochrones. Then, the progenitor lifetime of each white dwarf is derived by subtracting the cooling time to the age of the cluster. Taking into account the progenitor lifetime and the metallicity, the mass of the progenitor can be derived using stellar evolutionary sequences.

In this chapter we re-evaluate the available data that are currently being used to characterize the semi-empirical initial-final mass relationship (Ferrario et al. 2005; Kalirai et al. 2007b). We have followed the same procedure explained in chapter 2 to derive the initial and final masses of the white dwarfs, using in this case the age and metallicity of each cluster and the atmospheric parameters reported by different authors. As in the case of white dwarfs in common proper motion pairs we only consider white dwarfs of spectral type DA, i. e. with the presence of an envelope of Hydrogen at the top of their atmosphere, so that we can use the same cooling sequences as in chapter 2, those of Salaris et al. (2000), and keep consistency in our study. We have given special emphasis to re-calculating the recent data obtained for white dwarfs belonging to the open clusters M37 and M35. M37 contains more than 20 white dwarfs belonging to the representative range of initial masses between 
2.5 and $4.0 M_{\odot}$. These data has been useful to check the sensitivity of the results to different cooling sequences and stellar tracks. M35 is also interesting, since it constrains the high-mass end of the semi-empirical initial-final mass relationship.

\subsection{Available data}

\subsubsection{NGC 2099 (M37)}

Kalirai et al. (2005) performed spectroscopic observations of 24 white dwarfs belonging to NGC 2099. Kalirai et al. (2001) determined the metallicity of M37 and its age (520 Myr), though a more profound analysis led to a revision of the estimated metallicity $(Z=0.011)$ and, thus, to a modified value of the age, which turned out to be 650 Myr. As explained previously, the total age of a white dwarf can be expressed as the sum of the lifetime of its progenitor (time spent in the main-sequence and until the AGB phase), $t_{\text {prog, }}$, and its cooling time, $t_{\text {cool }}$. In this case, the total age of the white dwarfs is known, since it is equal to the cluster age, 650 Myr. Kalirai et al. (2005) derived $t_{\text {prog }}$ by subtracting the cooling time of each white dwarf to the total age, and estimated the mass of the progenitor star using the models of Hurley et al. (2000) for a metallicity of $Z=0.01$.

In Fig. 4.1 we show the initial and final masses obtained by Kalirai et al. (2005). For the sake of comparison, we represent also the theoretical initial-final mass relationship of Domínguez et al. (1999) for different metallicities, $Z=0.008,0.02$ and 0.011 , the latter corresponding to the cluster metallicity. Kalirai et al. (2005) consider that the theoretical initial-final mass relationship of Marigo (2001) fits properly their observational data in the range within 2.6 and $3.8 M_{\odot}-$ see Fig. 4.2. However, they do not take into account the white dwarfs in this range with low masses, since they consider that they could be field stars, although this has not been proved. Comparing both figures (Fig. 4.1 and 4.2) it can be noted that, in fact, the relation of Marigo (2001) seems to fit better the semi-empirical data, although the large dispersion of the data, probably due to the low quality of the spectra, prevents from drawing any conclusion. The behavior of these two theoretical relationships is quite different due to the particular considerations assumed in each model, mainly for what concerns mass-loss processes and the treatment of convection (see appendix B).

Using the photometric data $M_{V_{0}}$ and $(B-V)_{0}$ obtained by Kalirai et al. (2005) and the cooling sequences of Salaris et al. (2000) we can perform a first estimation of the white dwarf masses. $V_{0}$ can be derived considering the distance modulus of M37, $V_{0}-M_{V_{0}}=11.5$ (Kalirai et al. 2001) and the color should be corrected adding the reddening, $E(B-V)=0.23$. In Fig. 4.3 we show the cooling sequences of Salaris et al. (2000) for different white dwarf masses: $0.538 M_{\odot}, 0.606 M_{\odot}, 0.662 M_{\odot}, 0.768 M_{\odot}$, $0.862 M_{\odot}$, and $1 M_{\odot}$. If we overplot the photometric data of Kalirai et al. (2005) once it has been taken into account the distance modulus and the reddening - it 


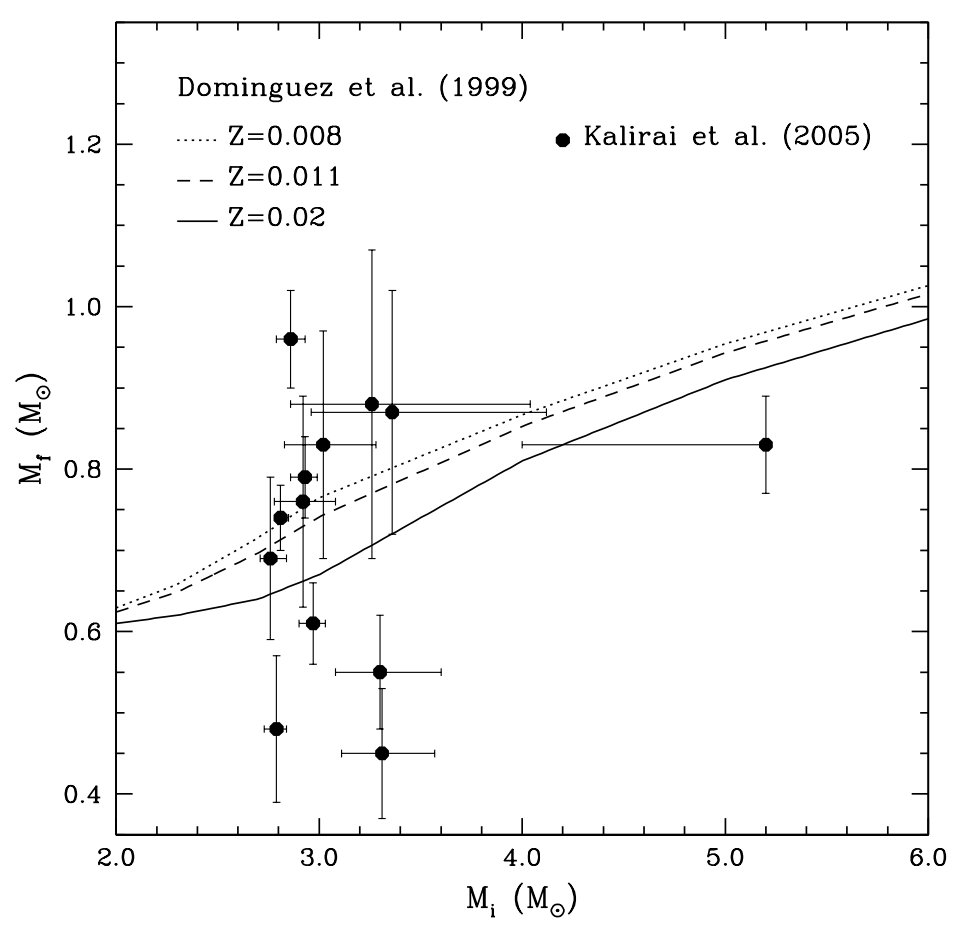

Figure 4.1: Final masses versus initial masses according to Domínguez et al. (1999) for different metallicities and the observational data obtained by Kalirai et al. (2005).

can be seen that the major part of points are located in this grid. Performing an interpolation for each case we can obtain a starting guess of the masses of the white dwarfs. We do not give these values, since the errors could not be calculated because we lack the observational errors of $V_{0}$ and $B-V$.

As previously mentioned, the best and most commonly used way to derive the masses of white dwarfs is to perform spectroscopic observations and then obtain their atmospheric parameters from the fit of the absorption lines to white dwarf models. Kalirai et al. (2005) determined $T_{\text {eff }}$ and $\log g$ using the method described in Bergeron et al. (1992). Then, they estimated the masses and cooling times using the cooling sequences of Fontaine et al. (2001), which consider a 50/50 mixture of carbon and oxygen for the core of the white dwarf with standard thick envelopes of hydrogen, $q(\mathrm{H})=10^{-4}$ and of helium, $q(\mathrm{He})=10^{-2}$ (see appendix A for more details). Since the observed spectra are not available we start our re-calculations from the atmospheric parameters reported by Kalirai et al. (2005), who in this case provide the errors. We have used the cooling sequences of Salaris et al. (2000) to derive the masses and the cooling times of the white dwarfs. Our results are detailed 


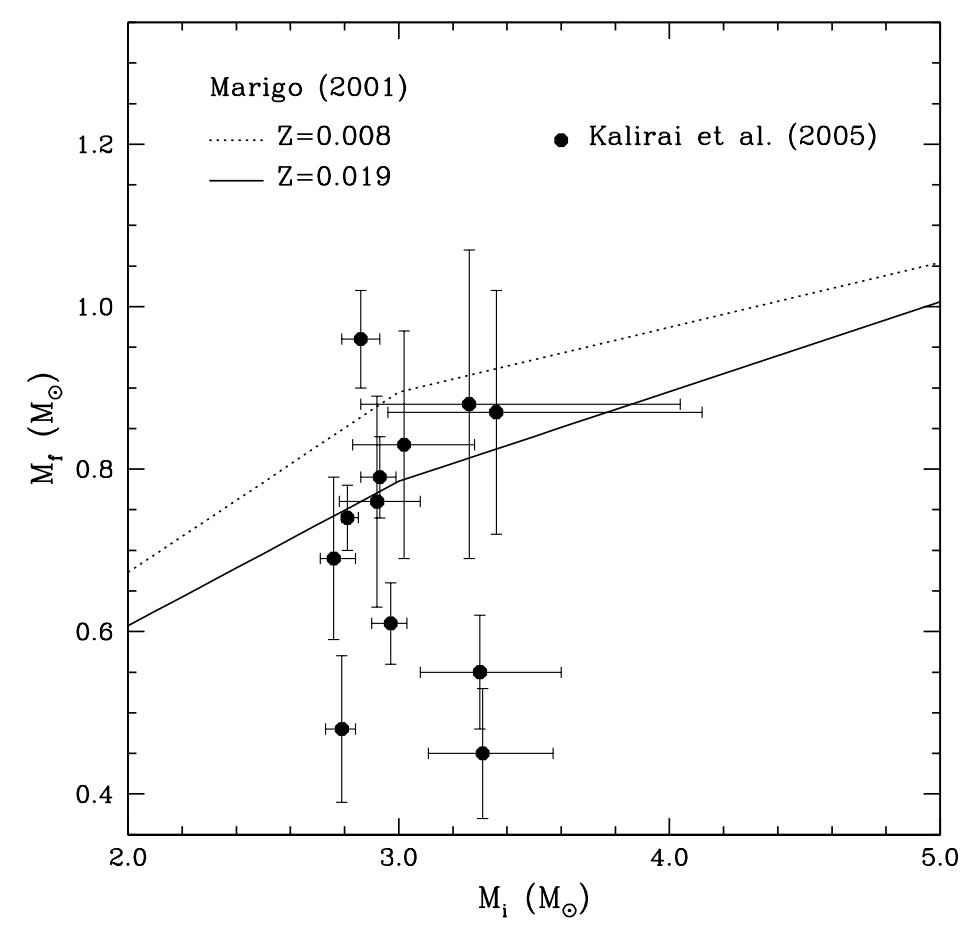

Figure 4.2: Final masses versus initial masses according to Marigo (2001) for different metallicities and the observational data obtained by Kalirai et al. (2005).

in Table 4.1. As can be noted, the masses obtained here are practically identical to those estimated by Kalirai et al. (2005), while the cooling times are generally shorter.

In Fig. 4.4 we show the differences between the cooling times obtained by Kalirai et al. (2005) and the ones derived using the cooling sequences of Salaris et al. (2000). Since the mass in each case differs in less than $5 \%$, we use the average value of both mass determinations in order to emphasize the differences in the cooling times. As can be seen, the values obtained in this work are generally shorter than those estimated by Kalirai et al. (2005). This is due to the fact that the models of Salaris et al. (2000) consider a white dwarf core with a larger concentration of oxygen at the center. Since the heat capacity of oxygen is smaller than that of carbon, this results in shorter cooling times for a given mass. As can be seen in Table 4.1, there are cases (WD15 and WD17) in which the cooling time obtained exceeds the total age of M37 (650 Myr). This may indicate that probably these white dwarfs are not members of the cluster. However, Kalirai et al. (2005) remark that WD15 and WD17 have masses that are too large to be considered field stars when the white dwarf mass 


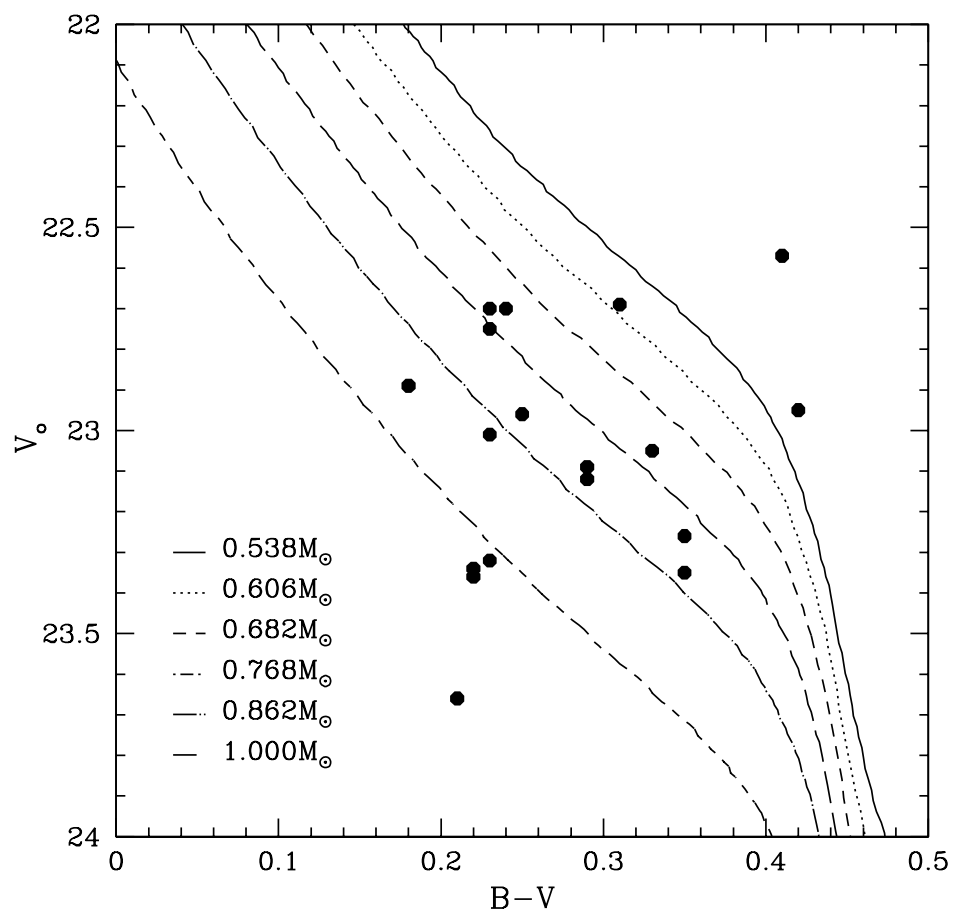

Figure 4.3: Color-magnitude diagram. The points are the observational data of Kalirai et al. (2005) and the lines are the cooling sequences of Salaris et al. (2000) for different white dwarf masses.

distribution of Madej et al. (2004) is taken into account. The only possibility that we can think of to explain the nature of these massive white dwarfs is that they have a core made of $\mathrm{O} / \mathrm{Ne}$, instead of $\mathrm{C} / \mathrm{O}$. For all the white dwarfs we have used the cooling sequences of Salaris et al. (2000), which assume a C/O core. However, this may not be appropriate for all the cases. Since WD15 and WD17 are massive, especially WD17, a core made of $\mathrm{O} / \mathrm{Ne}$ core will make plausible their membership to $\mathrm{M} 37$, since $\mathrm{O} / \mathrm{Ne}$ white dwarfs cool two times faster than $\mathrm{C} / \mathrm{O}$ white dwarfs (Althaus et al. 2007). It is worth noticing, though, that the cooling time of WD15 when considering the $\mathrm{C} / \mathrm{O}$ composition could be compatible with M37, if we take into a account the error bars. However, no definitive conclusions can be drawn in this case. In the case of WD6 and WD21 we were not able to calculate the masses and cooling times because Kalirai et al. (2005) could not determine and accurate value of $\log g$ due to the low $\mathrm{S} / \mathrm{N}$ of the spectra. In addition, WD1 and WD24 are at a wrong distance to be members of this cluster (Ferrario et al. 2005). For all these reasons, these six white dwarfs (WD1, WD6, WD15, WD17, WD21 and WD24) are 
Table 4.1: Atmospheric parameters, masses and cooling times obtained by Kalirai et al. (2005) and the same quantities when the cooling sequences of Salaris et al. (2000) are used.

\begin{tabular}{lcccccc}
\hline & & & \multicolumn{2}{c}{ Kalirai et al. $(2005)$} & \multicolumn{2}{c}{ This work } \\
\cline { 3 - 7 } Star & $T_{\text {eff }}$ & $\log g$ & $M_{\mathrm{f}}$ & $t_{\text {cool }}$ & $M_{\mathrm{f}}$ & $t_{\text {cool }}$ \\
& $(\mathrm{K})$ & $(\mathrm{dex})$ & $\left(M_{\odot}\right)$ & $(\mathrm{Myr})$ & $\left(M_{\odot}\right)$ & $(\mathrm{Myr})$ \\
\hline WD1 & $16500 \pm 800$ & $7.75 \pm 0.19$ & $0.48 \pm 0.09$ & $111 \pm 32$ & $0.48 \pm 0.07^{\star}$ & $92 \pm 21$ \\
WD2 & $19900 \pm 900$ & $8.11 \pm 0.16$ & $0.69 \pm 0.10$ & $108 \pm 36$ & $0.69 \pm 0.07$ & $93 \pm 19$ \\
WD3 & $18300 \pm 900$ & $8.23 \pm 0.21$ & $0.76 \pm 0.13$ & $179 \pm 64$ & $0.76 \pm 0.09$ & $152 \pm 34$ \\
WD4 & $16900 \pm 1100$ & $8.40 \pm 0.26$ & $0.87 \pm 0.15$ & $329 \pm 133$ & $0.86 \pm 0.12$ & $259 \pm 238$ \\
WD5 & $18300 \pm 1000$ & $8.33 \pm 0.22$ & $0.83 \pm 0.14$ & $224 \pm 85$ & $0.82 \pm 0.11$ & $192 \pm 78$ \\
WD6 & $17200 \pm 1600$ & $\ldots$ & $0.92 \pm 0.15$ & $300 \pm 100$ & $\ldots$ & $\ldots$ \\
WD7 & $17800 \pm 1400$ & $8.42 \pm 0.32$ & $0.88 \pm 0.19$ & $303 \pm 148$ & $0.88 \pm 0.14$ & $219 \pm 82$ \\
WD9 & $15300 \pm 400$ & $8.00 \pm 0.08$ & $0.61 \pm 0.05$ & $202 \pm 28$ & $0.61 \pm 0.03$ & $182 \pm 15$ \\
WD10 & $19300 \pm 400$ & $8.20 \pm 0.07$ & $0.74 \pm 0.04$ & $131 \pm 19$ & $0.74 \pm 0.03$ & $120 \pm 10$ \\
WD11 & $23000 \pm 600$ & $8.54 \pm 0.10$ & $0.96 \pm 0.06$ & $153 \pm 30$ & $0.98 \pm 0.04$ & $136 \pm 14$ \\
WD12 & $13300 \pm 1000$ & $7.91 \pm 0.12$ & $0.55 \pm 0.07$ & $313 \pm 70$ & $0.56 \pm 0.05$ & $239 \pm 43$ \\
WD13 & $18200 \pm 400$ & $8.27 \pm 0.08$ & $0.79 \pm 0.05$ & $183 \pm 28$ & $0.78 \pm 0.03$ & $167 \pm 16$ \\
WD14 & $11400 \pm 200$ & $7.73 \pm 0.16$ & $0.45 \pm 0.08$ & $318 \pm 61$ & $0.45 \pm 0.07^{\star}$ & $282 \pm 39$ \\
WD15 & $11300 \pm 200$ & $8.35 \pm 0.15$ & $0.83 \pm 0.09$ & $786 \pm 189$ & $0.82 \pm 0.07$ & $726 \pm 92$ \\
WD16 & $13100 \pm 500$ & $8.34 \pm 0.10$ & $0.83 \pm 0.06$ & $542 \pm 94$ & $0.82 \pm 0.05$ & $480 \pm 62$ \\
WD17 & $12300 \pm 400$ & $8.72 \pm 0.10$ & $1.05 \pm 0.05$ & $1346 \pm 256$ & $1.06 \pm 0.04^{\star}$ & $1501 \pm 240$ \\
WD21 & $17200 \pm 2000$ & $\ldots$ & $0.85 \pm 0.15$ & $261 \pm 100$ & $\ldots$ & $\ldots$ \\
WD24 & $18700 \pm 1100$ & $8.82 \pm 0.24$ & $1.11 \pm 0.13$ & $492 \pm 237$ & $1.14 \pm 0.12^{\star}$ & $490 \pm 157$ \\
\hline \hline
\end{tabular}

a The cooling time obtained by Kalirai et al. (2005) is longer than the age of the cluster.

$\mathrm{b}$ The cooling time obtained in this work is longer than the age of the cluster.

* Values obtained from extrapolation.

excluded from the semi-empirical initial-final mass relationship.

Once we have the cooling times of the white dwarfs, we can infer the progenitor lifetime of each white dwarf, $t_{\mathrm{prog}}$, by subtracting the cooling time to the total age of the white dwarf. As can be seen in Table 4.2 the progenitor lifetimes obtained here are longer. Obviously, this is due to the fact that we have obtained shorter cooling times. Once the progenitor lifetime of each white dwarf has been obtained, the stellar evolutionary tracks of Domínguez et al. (1999) with $Z=0.011$ can be used to estimate the mass of the progenitor. As can be seen in Table 4.2, the masses derived for the progenitors using the stellar evolutionary sequences of Domínguez et al. (1999) are typically about $7 \%$ smaller that the ones obtained by Kalirai et al. (2005) using the analytical models of Hurley et al. (2000).

In Fig. 4.5 we represent the data obtained using the cooling sequences of Salaris et al. (2000) and the stellar tracks of Domínguez et al. (1999) and the results obtained by Kalirai et al. (2005). We compare them with the theoretical initial-final mass rela- 


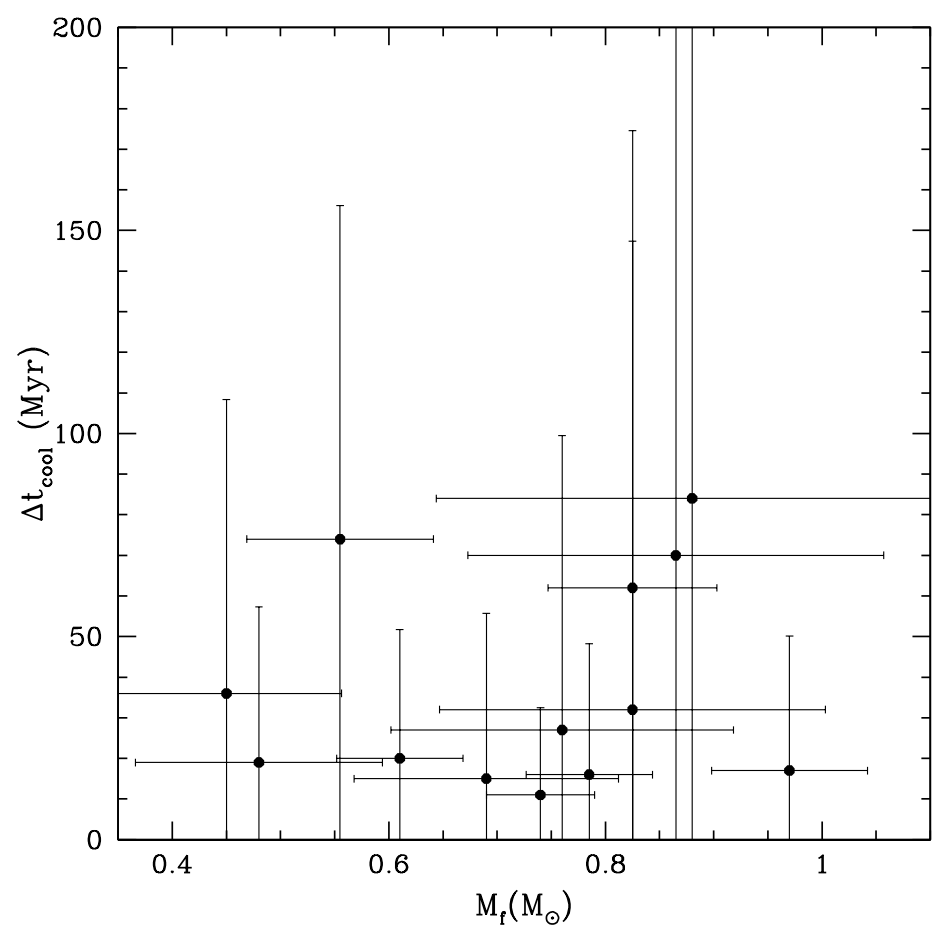

Figure 4.4: Plot of the difference between the cooling times obtained by Kalirai et al. (2005) and the ones derived in this work using the cooling sequences of Salaris et al. (2000), versus the average mass of the white dwarf.

tionships of Domínguez et al. (1999) (left panel) for consistency with the theoretical tracks used in our calculations, and with the relation of Marigo (2001) (right panel). In general, the progenitors derived using the stellar models of Domínguez et al. (1999) turned out to be less massive. The semi-empirical data obtained in both Kalirai et al. (2005) and in this work present large error bars and a considerable dispersion. The data is not fitted by any of the theoretical relationships, although the theoretical initial-final mass relationship of Marigo (2001) seems to be in better agreement with the observational data. Thus, it is difficult to identify if such a dispersion is real or it is caused by the uncertainties introduced during the calculation procedure. It is also hard to explain why stars with similar initial masses can evolve into white dwarfs with such different masses. Moreover, in principle, this fact should not be a consequence of the different metallicities of the progenitors, since all of them belong to the same cluster and therefore, had the same metallicity $(Z=0.011)$. Thus, the observational data seem to support the hypothesis that these differences - at least for the white dwarfs in this cluster — should be caused by other physical phenomena, like 
Table 4.2: Progenitor lifetimes, final masses and initial masses obtained by Kalirai et al. (2005) and the ones derived in this work using the cooling sequences of Salaris et al. (2000) and the stellar evolutionary tracks of Domínguez et al. (1999).

\begin{tabular}{ccccccc}
\hline \hline \multirow{3}{*}{ Star } & \multicolumn{3}{c}{ Kalirai et al. $(2005)$} & \multicolumn{3}{c}{ This work } \\
\cline { 2 - 7 } & $\begin{array}{c}t_{\text {prog }} \\
(\mathrm{Myr})\end{array}$ & $\begin{array}{c}M_{\mathrm{f}} \\
\left(M_{\odot}\right)\end{array}$ & $\begin{array}{c}M_{\mathrm{i}} \\
\left(M_{\odot}\right)\end{array}$ & $\begin{array}{c}t_{\text {prog }} \\
(\mathrm{Myr})\end{array}$ & $\begin{array}{c}M_{\mathrm{f}} \\
\left(M_{\odot}\right)\end{array}$ & $\begin{array}{c}M_{\mathrm{i}} \\
\left(M_{\odot}\right)\end{array}$ \\
\hline WD2 & $542 \pm 36$ & $0.69 \pm 0.10$ & $2.76_{-0.05}^{+0.08}$ & $557 \pm 19$ & $0.69 \pm 0.07$ & $2.72_{-0.03}^{+0.03}$ \\
WD3 & $471 \pm 64$ & $0.76 \pm 0.13$ & $2.92_{-0.14}^{+0.16}$ & $498 \pm 34$ & $0.76 \pm 0.09$ & $2.82_{-0.06}^{+0.06}$ \\
WD4 & $321 \pm 133$ & $0.87 \pm 0.15$ & $3.36_{-0.40}^{+0.76}$ & $391 \pm 238$ & $0.86 \pm 0.12$ & $3.06_{-0.45}^{+1.22}$ \\
WD5 & $426 \pm 85$ & $0.83 \pm 0.14$ & $3.02_{-0.19}^{+0.26}$ & $458 \pm 78$ & $0.82 \pm 0.11$ & $2.90_{-0.15}^{+0.19}$ \\
WD7 & $347 \pm 148$ & $0.88 \pm 0.19$ & $3.26_{-0.40}^{+0.78}$ & $431 \pm 82$ & $0.88 \pm 0.14$ & $2.96_{-0.16}^{+0.22}$ \\
WD9 & $448 \pm 28$ & $0.61 \pm 0.05$ & $2.97_{-0.07}^{+0.06}$ & $468 \pm 15$ & $0.61 \pm 0.03$ & $2.88_{-0.03}^{+0.03}$ \\
WD10 & $519 \pm 19$ & $0.74 \pm 0.04$ & $2.81_{-0.02}^{+0.04}$ & $530 \pm 10$ & $0.74 \pm 0.03$ & $2.76_{-0.02}^{+0.02}$ \\
WD11 & $498 \pm 30$ & $0.96 \pm 0.06$ & $2.86_{-0.07}^{+0.07}$ & $514 \pm 14$ & $0.98 \pm 0.04$ & $2.80_{-0.02}^{+0.03}$ \\
WD12 & $337 \pm 70$ & $0.55 \pm 0.07$ & $3.30_{-0.22}^{+0.30}$ & $411 \pm 43$ & $0.56 \pm 0.05$ & $3.01_{-0.10}^{+0.12}$ \\
WD13 & $467 \pm 28$ & $0.79 \pm 0.05$ & $2.93_{-0.07}^{+0.06}$ & $483 \pm 16$ & $0.78 \pm 0.03$ & $2.86_{-0.03}^{+0.03}$ \\
WD14 & $332 \pm 61$ & $0.45 \pm 0.08$ & $3.31_{-0.20}^{+0.26}$ & $368 \pm 39$ & $0.45 \pm 0.07$ & $3.12_{-0.11}^{+0.12}$ \\
WD16 & $108 \pm 94$ & $0.83 \pm 0.06$ & $5.20_{-1.20}^{+?}$ & $170 \pm 62$ & $0.82 \pm 0.05$ & $4.10_{-0.44}^{+0.80}$ \\
\hline \hline
\end{tabular}
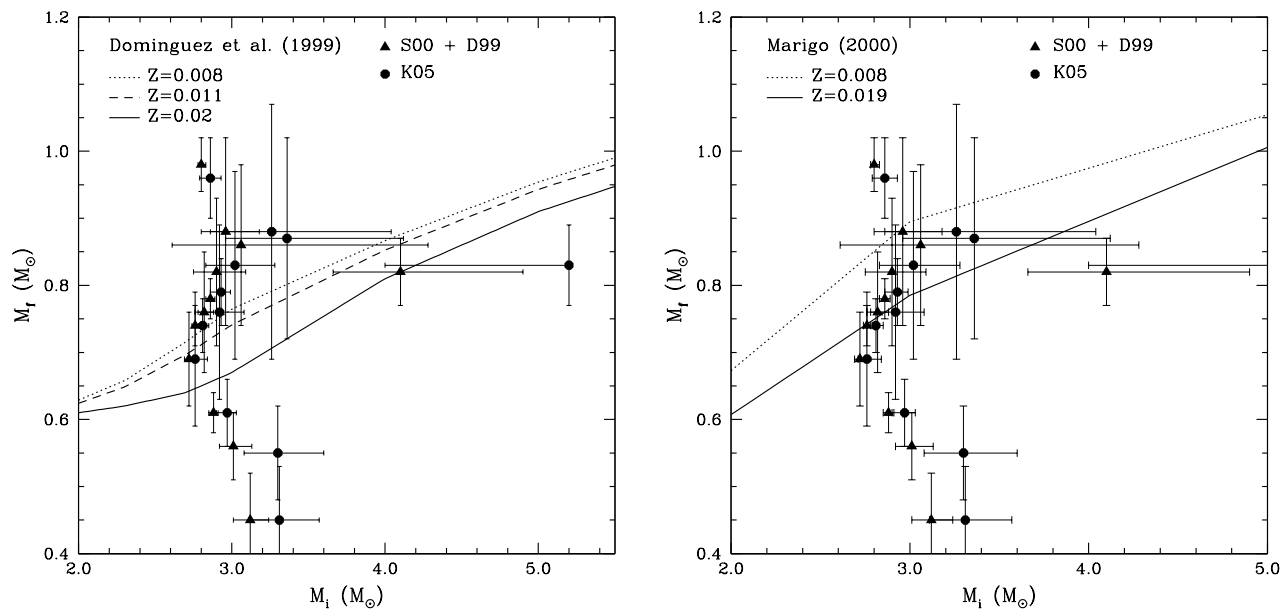

Figure 4.5: Final versus initial masses obtained by Kalirai et al. (2005) and the ones derived in this work using the cooling sequences of Salaris et al. (2000) and the stellar tracks of Domínguez et al. (1999), compared with different theoretical functions. 


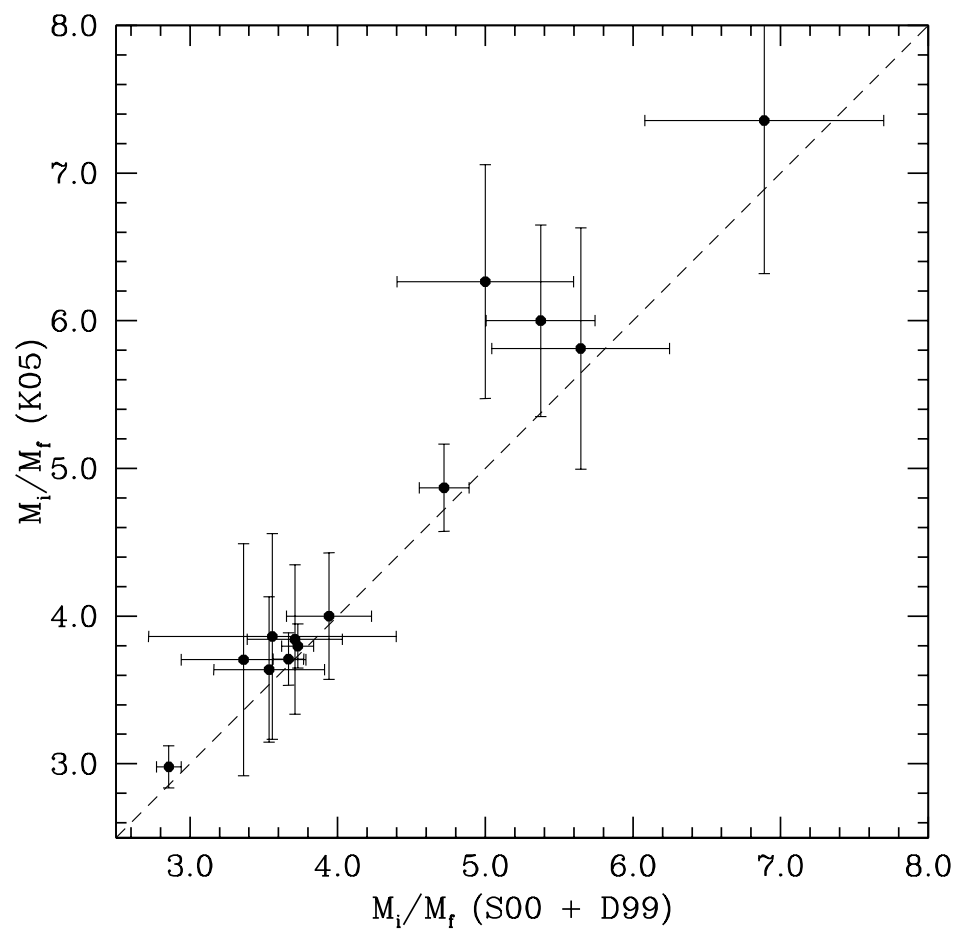

Figure 4.6: Correspondence between the semi-empirical relation of Kalirai et al. (2005), and that obtained in this work using the cooling sequences of Salaris et al. (2000) and the stellar evolutionary models of Domínguez et al. (1999).

rotation, magnetic fields... It is worth mentioning that from an observational point of view it is impossible to know the exact stratification and internal composition of a white dwarf, except when it is a pulsating star (see e. g. Silvotti et al. 2005 and references therein), which is not the case. When the masses and cooling times of white dwarfs are derived from the same cooling sequences we are implicitly assuming that they have the same internal composition, which is probably not true. Moreover, the thicknesses of the $\mathrm{H}$ envelope and He buffer of a white dwarf are very important in their evolution, since they control its cooling rate, and these may be also different from one star to another. Using the same cooling sequences for all white dwarfs in the cluster may introduce some dispersion in the results, since the progenitor lifetimes, and by extension the initial masses of the progenitors are very dependent on the cooling times derived. Since we do not know the properties of the interiors and the characteristics of the outer layers of these white dwarfs, we have prefered to assume the same cooling sequences everywhere for the sake of consistency.

In order to check whether or not there is a systematic bias between the data 
Table 4.3: Initial masses obtained using the stellar models of Domínguez et al. (1999) labelled as D99 — using the analytical fits of Hurley et al. (2000) — labelled as H00 — and using the stellar tracks of Pietrinferni et al. (2004) without and with overshooting - labelled as P04 and P04o, respectively.

\begin{tabular}{|c|c|c|c|c|c|}
\hline Star & $\begin{array}{c}t_{\text {prog }} \\
(\mathrm{Myr})\end{array}$ & $\begin{array}{c}M_{\mathrm{i}}(\mathrm{D} 99) \\
\quad\left(M_{\odot}\right)\end{array}$ & $\begin{array}{c}M_{\mathrm{i}}(\mathrm{H} 00) \\
\quad\left(M_{\odot}\right)\end{array}$ & $\begin{array}{c}M_{\mathrm{i}}(\mathrm{P} 04) \\
\quad\left(M_{\odot}\right)\end{array}$ & $\begin{array}{c}M_{\mathrm{i}}(\mathrm{P} 04 \mathrm{o}) \\
\quad\left(M_{\odot}\right)\end{array}$ \\
\hline WD2 & $557 \pm 19$ & $2.72_{-0.03}^{+0.03}$ & $2.75_{-0.03}^{+0.04}$ & $2.35_{-0.02}^{+0.02}$ & $2.57_{-0.03}^{+0.03}$ \\
\hline WD3 & $498 \pm 34$ & $2.82_{-0.06}^{+0.06}$ & $2.86_{-0.07}^{+0.07}$ & $2.44_{-0.05}^{+0.06}$ & $2.66_{-0.05}^{+0.06}$ \\
\hline WD4 & $391 \pm 238$ & $3.06_{-0.45}^{+1.22}$ & $3.18_{-0.55}^{+1.35}$ & $2.66_{-0.38}^{+1.11}$ & $2.88_{-0.40}^{+1.17}$ \\
\hline WD5 & $458 \pm 78$ & $2.90_{-0.15}^{+0.19}$ & $2.95_{-0.16}^{+0.28}$ & $2.51_{-0.13}^{+0.38}$ & $2.73_{-0.13}^{+0.17}$ \\
\hline WD7 & $431 \pm 82$ & $2.96_{-0.16}^{+0.22}$ & $3.01_{-0.18}^{+0.34}$ & $2.57_{-015}^{+0.19}$ & $2.79_{-0.15}^{+0.20}$ \\
\hline WD9 & $468 \pm 15$ & $2.88_{-0.03}^{+0.03}$ & $2.92_{-0.03}^{+0.03}$ & $2.50_{-0.03}^{+0.03}$ & $2.71_{-0.03}^{+0.03}$ \\
\hline WD10 & $530 \pm 10$ & $2.76_{-0.02}^{+0.02}$ & $2.80_{-0.02}^{+0.02}$ & $2.39_{-0.02}^{+0.02}$ & $2.61_{-0.02}^{+0.02}$ \\
\hline WD11 ${ }^{\mathrm{b}}$ & $514 \pm 14$ & $2.80_{-0.02}^{+0.03}$ & $2.83_{-0.03}^{+0.03}$ & $2.42_{-0.02}^{+0.02}$ & $2.63_{-0.02}^{+0.02}$ \\
\hline WD12 & $411 \pm 43$ & $3.01_{-0.09}^{+0.12}$ & $3.09_{-0.14}^{+0.18}$ & $2.61_{-0.09}^{+0.10}$ & $2.83_{-0.09}^{+0.11}$ \\
\hline WD13 & $483 \pm 16$ & $2.86_{-0.03}^{+0.03}$ & $2.89_{-0.03}^{+0.03}$ & $2.47_{-0.03}^{+0.03}$ & $2.69_{-0.03}^{+0.03}$ \\
\hline WD14 & $368 \pm 39$ & $3.12_{-0.11}^{+0.12}$ & $3.24_{-0.18}^{+0.017}$ & $2.71_{-0.09}^{+0.05}$ & $2.93_{-0.10}^{+0.05}$ \\
\hline WD16 & $170 \pm 62$ & $4.10_{-0.44}^{+0.80}$ & $4.35_{-0.52}^{+0.84}$ & $3.61_{-0.41}^{+0.72}$ & $3.88_{-0.43}^{+0.77}$ \\
\hline
\end{tabular}

obtained here and the data of Kalirai et al. (2005) in Fig. 4.6 we compare the different semi-empirical initial-final mass relationships by representing the ratio between the initial and the final mass obtained in both works for each of the white dwarfs of this cluster. As it can be appreciated in Fig. 4.6, there is a good correspondence between both semi-empirical initial-final mass relationships, except for very large ratios. Thus, it seems that in the large-mass domain the white dwarf cooling sequences of Salaris et al. (2000) yield shorter cooling times than those of Fontaine et al. (2001), which result in longer progenitor lifetimes, thus obtaining smaller initial masses. This, in turn, is a consequence of the different oxygen concentration in the core. Hence, having reliable cooling sequences, with the appropriate internal composition, turns out to be crucial in deriving a more precise semi-empirical initial-final mass relationship.

However, this issue - the adopted $\mathrm{C} / \mathrm{O}$ ratio in the core of the white dwarf is not the only one that influences the determinations of the initial and final masses. The other relevant factor is the pre-white dwarf stellar evolutionary tracks adopted. This can be checked by adopting the same set of stellar models used in Kalirai et al. (2005), namely, the analytical fits of Hurley et al. (2000). The results obtained using this set of pre-white dwarf stellar models are detailed in column 3 of Table 4.3. In Fig. 4.7 we show the semi-empirical relations obtained using the cooling sequences 

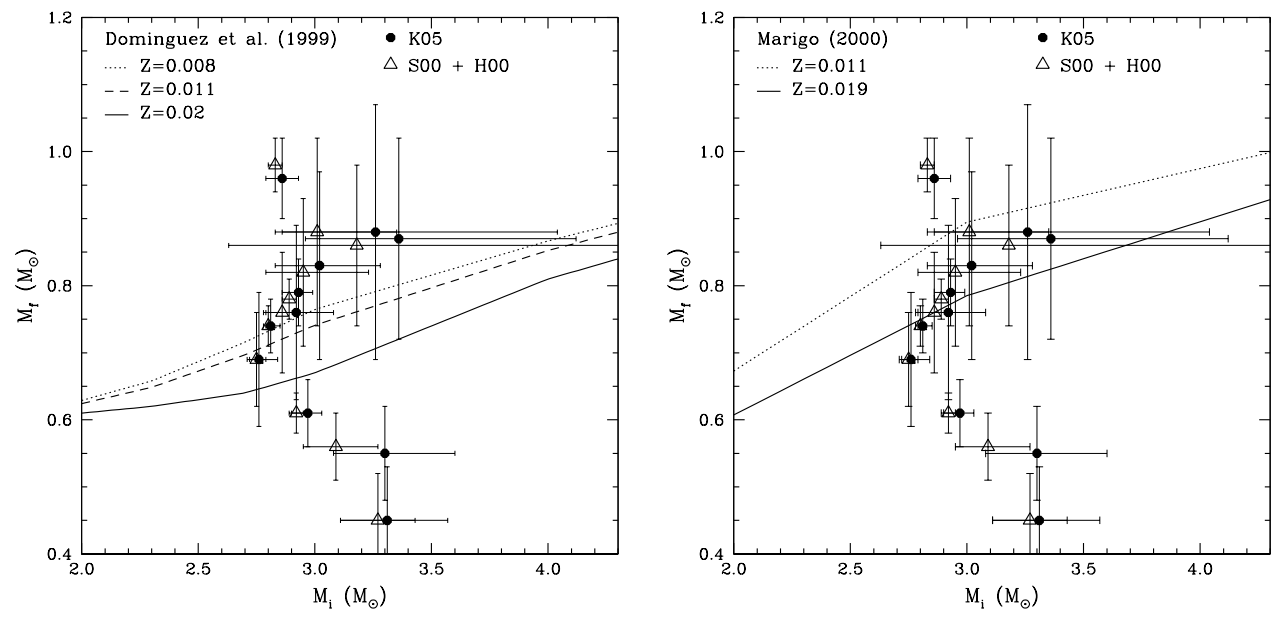

Figure 4.7: Final masses versus initial masses obtained using the cooling sequences of Salaris et al. (2000) and the evolutive models of Hurley et al. (2000), compared with the data of Kalirai et al. (2005) and the theoretical relations of Domínguez et al. (1999) - left panel and Marigo (2001) — right panel.

of Salaris et al. (2000) and the evolutive models of Hurley et al. (2000). For the sake of comparison we plot also the data obtained by Kalirai et al. (2005), who used the cooling sequences of Fontaine et al. (2001). In this case, the difference is only a consequence of using different cooling sequences, since the stellar tracks are the same. The initial masses obtained are smaller in the case of using the cooling sequences of Salaris et al. (2000), because they give shorter cooling times, and thus, the progenitor lifetimes are longer and the initial masses smaller. As can be seen the largest differences between our results and those obtained by Kalirai et al. (2005) are due to the different cooling sequences used, rather than to the different set of pre-white dwarf evolutionary tracks.

The situation is not the same when the effects of overshooting are taken in due account. The last two columns of Table 4.3 show the results when the stellar evolutionary sequences of Pietrinferni et al. (2004) are used. In chapter 1 we highlighted that convection was a parameter of paramount importance during the evolution of a star. Pietrinferni et al. (2004) carried out two sets of computations with the same physical assumptions and inputs, but with and without considering overshooting. As explained in appendix B, for the same initial mass a more massive white dwarf is obtained when overshooting is taken into account, since the core can grow further. At the same time, the main-sequence lifetimes considering overshooting are shorter, and this is the why we have obtained larger initial masses in that case - see Table 4.3 . 
Table 4.4: Atmospheric parameters, masses and cooling times obtained by Williams et al. (2004), and masses and cooling times derived in this work using the cooling sequences of Salaris et al. (2000).

\begin{tabular}{|c|c|c|c|c|c|c|}
\hline \multirow[b]{2}{*}{ Star } & \multirow[b]{2}{*}{$\begin{array}{l}T_{\text {eff }} \\
(\mathrm{K})\end{array}$} & \multirow[b]{2}{*}{$\begin{array}{l}\log g \\
(\operatorname{dex})\end{array}$} & \multicolumn{2}{|c|}{ Williams et al. (2004) } & \multicolumn{2}{|c|}{ This work } \\
\hline & & & $\begin{array}{c}M_{\mathrm{f}} \\
\left(M_{\odot}\right)\end{array}$ & $\begin{array}{c}t_{\text {cool }} \\
(\mathrm{Myr})\end{array}$ & $\begin{array}{c}M_{\mathrm{f}} \\
\left(M_{\odot}\right)\end{array}$ & $\begin{array}{c}t_{\text {cool }} \\
(\mathrm{Myr})\end{array}$ \\
\hline LAWDS1 & $32400 \pm 512$ & $8.40 \pm 0.12$ & $0.89 \pm 0.07$ & $24 \pm 5$ & $0.89 \pm 0.06$ & $23 \pm 7$ \\
\hline LAWDS2 ${ }^{\mathrm{a}}$ & $32700 \pm 603$ & $8.34 \pm 0.08$ & $0.86 \pm 0.05$ & $17.1 \pm 0.3$ & $0.85 \pm 0.04$ & $16.7 \pm 0.1$ \\
\hline LAWDS5 & $52600 \pm 1160$ & $8.24 \pm 0.09$ & $0.82 \pm 0.04$ & $1.44 \pm 0.04$ & $0.82 \pm 0.04$ & $2.2 \pm 0.1$ \\
\hline LAWDS6 & $55200 \pm 897$ & $8.28 \pm 0.06$ & $0.85 \pm 0.04$ & $1.24 \pm 0.03$ & $0.84 \pm 0.03$ & $2.0 \pm 0.1$ \\
\hline LAWDS11 ${ }^{\mathrm{b}, \mathrm{c}}$ & $19900 \pm 2792$ & $8.48 \pm 0.37$ & $0.93 \pm 0.16$ & $194 \pm 90$ & $0.93 \pm 0.16$ & $196 \pm 94$ \\
\hline LAWDS15 & $29900 \pm 318$ & $8.48 \pm 0.06$ & $0.93 \pm 0.04$ & $49 \pm 4$ & $0.94 \pm 0.03$ & $45 \pm 4$ \\
\hline LAWDS22 & $54400 \pm 1203$ & $8.04 \pm 0.12$ & $0.72 \pm 0.06$ & $1.48 \pm 0.08$ & $0.72 \pm 0.04$ & $2.5 \pm 0.2$ \\
\hline LAWDS27 ${ }^{\mathrm{a}}$ & $30500 \pm 397$ & $8.52 \pm 0.06$ & $0.96 \pm 0.03$ & $47.4 \pm 0.6$ & $0.98 \pm 0.03$ & $48 \pm 4$ \\
\hline
\end{tabular}

a New data from Ferrario et al. (2005).

b The cooling time obtained by Williams et al. (2004) is longer than the age of the cluster.

c The cooling time obtained in this work is longer than the age of the cluster.

Considering the results obtained from using different cooling sequences and stellar tracks, we conclude that the most important factors influencing the initial-final mass relationship are the prescriptions adopted for convection in the pre-white dwarf evolutionary phase and the internal composition adopted in the white dwarf cooling sequences.

\subsubsection{NGC2168 (M35)}

Williams et al. (2004) performed spectroscopic observations of a sample of white dwarf candidates belonging to NGC2168 (M35). Previously, Barrado y Navascués et al. (2001) studied this cluster and estimated its metal abundance, $[\mathrm{Fe} / \mathrm{H}]=-0.21 \pm$ 0.10 , distance modulus, $(m-M)=10.16 \pm 0.01$, reddening, $E(B-V)=0.198 \pm 0.008$, and age, $160 \pm 40 \mathrm{Myr}$. Thus, the metallicity of M35 is $Z=0.012$. More recently, von Hippel (2005) derived a cluster age of $150 \pm 60$ Myr. For consistency with Williams et al. (2004) we use this last value. Williams et al. (2004) used the cooling sequences of Wood (1995), which consider C/O core white dwarfs with $10^{-4}$ fractional hydrogen and $10^{-2}$ fractional helium layers. To calculate the initial masses, the authors used the stellar isochrones of Girardi et al. (2002) with modest convective overshoot for two values of the metallicity $Z=0.008$ and 0.019 , and without convective overshoot with $Z=0.019$. It is worth to mention that the cooling sequences of Wood (1995) are not as accurate as the cooling sequences of Salaris et al. (2000), which include an 


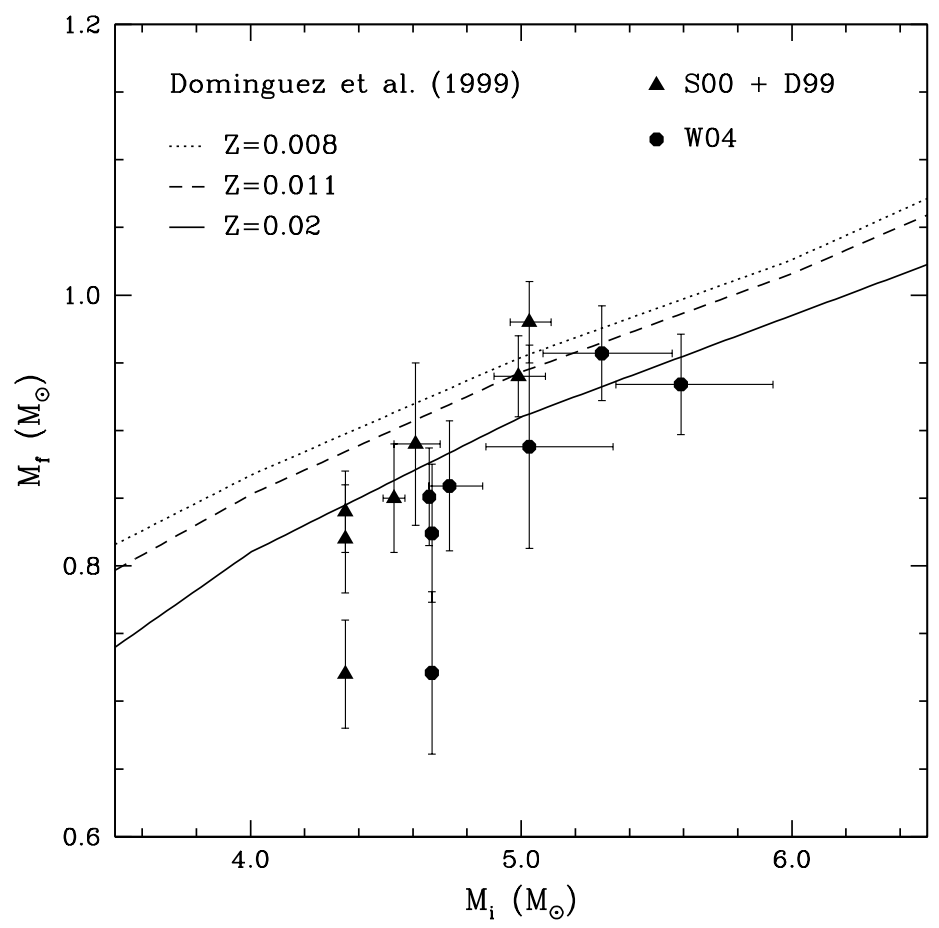

Figure 4.8: Initial-final mass relationship obtained by Williams et al. (2004) and that obtained in this work.

accurate treatment of phase separation upon crystallization of the $\mathrm{C} / \mathrm{O}$ core, together with up-to-date input physics suitable for computing white dwarf evolution.

We have followed the same procedure described in the previous section in order to re-calculate the initial and final masses. For white dwarfs LAWDS2 and LAWDS27 we have used the atmospheric parameters reported in Ferrario et al. (2005) corresponding to new data. As can be seen in Table 4.4, the white dwarfs of this cluster are more massive than those of M37. This is due to the fact that M35 is younger and thus, the stars that have had time enough to become white dwarfs are the ones which had a larger mass in the main sequence. For LAWDS 11 we have obtained a cooling time larger than the age of the cluster. This result was obtained also by Williams et al. (2004), who considered that this star may not be a cluster member.

In Fig. 4.8 it can be seen that the relationship obtained here is rather similar to that of Williams et al. (2004). Comparing these results with those of M37 it can be noted that in this region of masses the theoretical relations show a better agreement with the observational data. It is worth mentioning that we have taken into account all the white dwarfs considered by Williams et al. (2004) in their study, even though 
Table 4.5: Comparison of the initial masses obtained by Williams et al. (2004) and those obtained here.

\begin{tabular}{|c|c|c|c|c|c|}
\hline \multirow{3}{*}{ Star } & \multicolumn{3}{|c|}{ Williams et al. (2004) } & \multicolumn{2}{|c|}{ This work } \\
\hline & $t_{\text {prog }}(\mathrm{Myr})$ & $M_{\mathrm{i}}\left(M_{\odot}\right)$ & $M_{\mathrm{i}}\left(M_{\odot}\right)$ & $t_{\text {prog }}(\mathrm{Myr})$ & $M_{\mathrm{i}}\left(M_{\odot}\right)$ \\
\hline & & $Z=0.008$ & $Z=0.019$ & & $Z=0.011$ \\
\hline LAWDS1 & $126 \pm 6$ & $5.00_{-0.16}^{+0.31}$ & $5.08_{-0.15}^{+0.29}$ & $127 \pm 6$ & $4.61_{-0.08}^{+0.09}$ \\
\hline LAWDS2 ${ }^{\mathrm{a}}$ & $133 \pm 3$ & $4.73_{-0.08}^{+0.12}$ & & $133 \pm 3$ & $4.53_{-0.04}^{+0.08}$ \\
\hline LAWDS5 & $148.56 \pm 0.04$ & $4.63_{-0.001}^{+0.001}$ & $4.73_{-0.001}^{+0.002}$ & $147.8 \pm 0.1$ & $4.35_{-0.001}^{+0.001}$ \\
\hline LAWDS6 & $148.76 \pm 0.03$ & $4.63_{-0.001}^{+0.001}$ & $4.73_{-0.001}^{+0.001}$ & $148.0 \pm 0.1$ & $4.35_{-0.001}^{+0.001}$ \\
\hline LAWDS15 & $101 \pm 4$ & $5.57_{-0.24}^{+0.034}$ & $5.62_{-0.22}^{+0.31}$ & $104 \pm 5$ & $4.99_{-0.09}^{+0.10}$ \\
\hline LAWDS22 & $148.52 \pm 0.08$ & $4.63_{-0.003}^{+0.003}$ & $4.735_{-0.002}^{+0.003}$ & $147.5 \pm 0.2$ & $4.35_{-0.002}^{+0.002}$ \\
\hline LAWDS27a & $102 \pm 1$ & $5.30_{-0.22}^{+0.263}$ & $2.00-0.002$ & $102 \pm 4$ & $5.03_{-0.07}^{+0.082}$ \\
\hline
\end{tabular}

a New data from Ferrario et al. (2005).

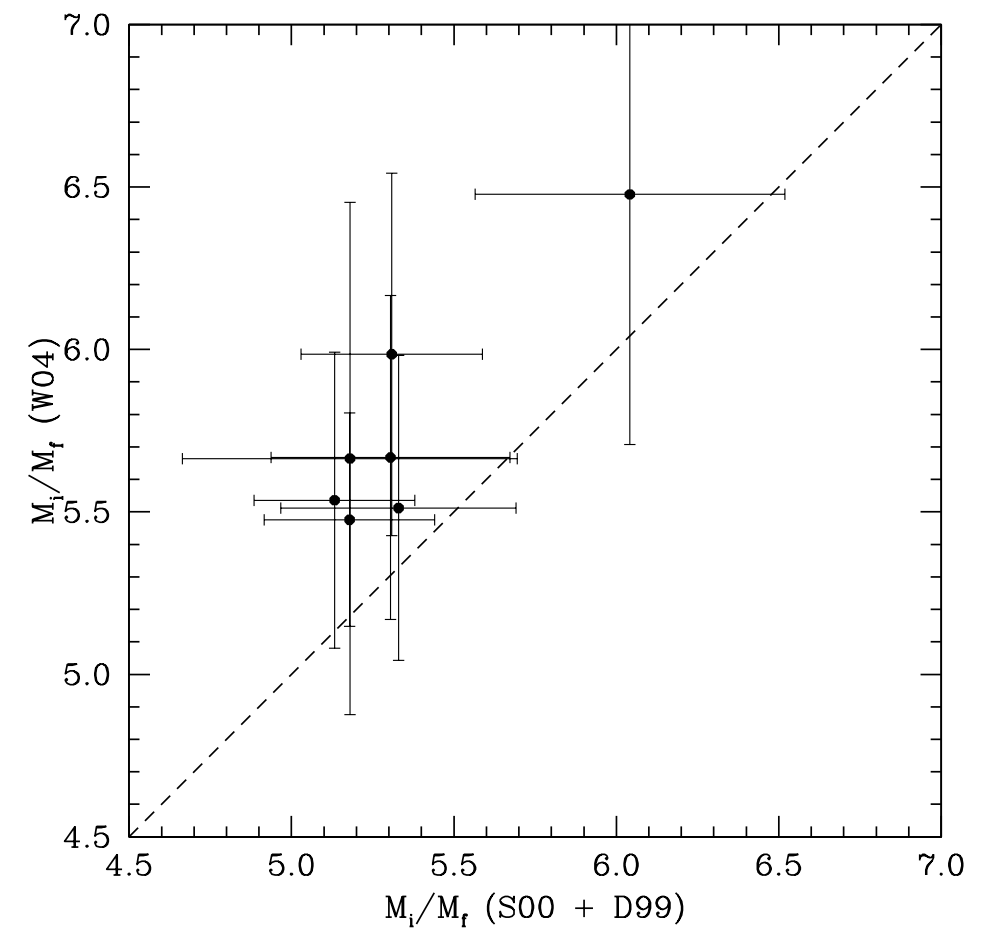

Figure 4.9: Comparison between the initial-final mass relationships obtained by Williams et al. (2004) and in this work. 
some of them could be eventually field stars or escaped cluster white dwarfs. In particular, LAWDS 22 might not be a very representative point in the initial-final mass relationship since according to Williams et al. (2004) the spectral fit of the spectrum of this star did not converge satisfactorily despite the high signal-to-noise ratio. On the other hand, this star is a close visual binary with a redder companion and hence, its spectrum should be contaminated.

In Fig. 4.9 we show the ratio between the semi-empirical initial-final mass relationships obtained by Williams et al. (2004) and that derived in this work. In this case the cooling times obtained were very similar, so, the lack of correspondence between our results and the ones obtained by Williams et al. (2004) are caused by the different assumptions considered in the stellar evolutionary tracks of Girardi et al. (2002) and Domínguez et al. (1999) (see appendix B). It is interesting to realize that, in general, the data approach adopted by Williams et al. (2004) results in larger initial masses for the same white dwarf mass.

\subsubsection{Other available data}

We have also re-calculated the initial and final masses of the available data that are currently being used to define the semi-empirical initial final mass relationship. For instance, NGC 2516 is another well studied cluster. In this case we used the atmospheric parameters reported by Koester \& Reimers (1996) for the white dwarfs of the cluster. The age of the cluster is $141 \pm 2 \mathrm{Myr}$ (Meynet et al. 1993). This cluster has a solar metallicity according to Jeffries et al. (1998).

The cluster data for NGC 3532 are from Koester \& Reimers (1993), although we have used the latest results of Koester \& Reimers (1996) and those reported in Ferrario et al. (2005). According to Ferrario et al. (2005) the age of this cluster is $300 \pm 150$, and the metallicity is $[\mathrm{Fe} / \mathrm{H}]=-0.022$ (Twarog et al. 1997) or $[\mathrm{Fe} / \mathrm{H}]=$ -0.02 (Chen et al. 2003), so we have used the tracks corresponding to $Z=0.019$. We have also included the data for the three white dwarfs reported in Reimers \& Koester (1989), although the resolution is lower.

Hyades and Praesepe are also very well studied clusters. We have used the data of Claver et al. (2001) for the Hyades and part of the Praesepe sample. We have included also two stars studied by Dobbie et al. (2004) and some recent results of six new stars (Dobbie et al. 2006). According to von Hippel (2005), the Hyades cluster has an age of $625 \pm 50 \mathrm{Myr}$. We assume the same value for Praesepe, since both belong to the same Hyades supercluster. The metallicity is $[\mathrm{Fe} / \mathrm{H}]=0.13$ according to Chen et al. (2003), so we use the stellar tracks corresponding to $Z=0.027$.

WD0349+247 is the only known Pleiades white dwarf. We have used the atmospheric parameters derived by Dobbie et al. (2006). The age of the Pleiades is $125 \pm 25$ Myr according to Ferrario et al. (2005), which is in good agreement with the value of 119 Myr estimated by Martín et al. (2001) using lithium abundances and Kband photometry of very low-mass members. The metallicity of the Pleiades derived 
Table 4.6: Our results based in other available data.

\begin{tabular}{|c|c|c|c|c|c|c|}
\hline WD & $\begin{array}{l}T_{\text {eff }} \\
(\mathrm{K})\end{array}$ & $\begin{array}{l}\log g \\
(\operatorname{dex})\end{array}$ & $\begin{array}{c}M_{\mathrm{f}} \\
M_{\odot}\end{array}$ & $\begin{array}{c}t_{\text {cool }} \\
(\mathrm{Gyr})\end{array}$ & $\begin{array}{c}t_{\text {prog }} \\
\text { (Gyr) }\end{array}$ & $\begin{array}{c}M_{\mathrm{i}} \\
\left(M_{\odot}\right)\end{array}$ \\
\hline \multicolumn{7}{|l|}{ Clusters } \\
\hline \multicolumn{7}{|l|}{ NGC 2516} \\
\hline 2516-WD1 & $28170 \pm 310$ & $8.48 \pm 0.17$ & $0.95 \pm 0.08$ & $0.060 \pm 0.012$ & $0.081 \pm 0.012$ & $5.55_{-0.29}^{+0.39}$ \\
\hline 2516-WD2 & $34200 \pm 610$ & $8.60 \pm 0.11$ & $1.01 \pm 0.04^{\star}$ & $0.035 \pm 0.008$ & $0.106 \pm 0.008$ & $5.03_{-0.13}^{+0.14}$ \\
\hline 2516-WD3 & $26870 \pm 330$ & $8.55 \pm 0.07$ & $0.99 \pm 0.03$ & $0.082 \pm 0.005$ & $0.059 \pm 0.005$ & $6.38_{-0.24}^{+0.13}$ \\
\hline 2516-WD5 & $30760 \pm 420$ & $8.70 \pm 0.12$ & $1.07 \pm 0.05^{\star}$ & $0.074 \pm 0.014$ & $0.067 \pm 0.014$ & $6.02_{-0.47}^{+0.68}$ \\
\hline \multicolumn{7}{|l|}{ NGC 3532} \\
\hline 3532-WD1 & $28000 \pm 2000$ & $8.45 \pm 0.45$ & $0.92 \pm 0.20$ & $0.055 \pm 0.036$ & $0.245 \pm 0.154$ & $3.70_{-0.55}^{+1.63}$ \\
\hline 3532-WD5 & $28500 \pm 2000$ & $7.8 \pm 0.3$ & $0.55 \pm 0.11$ & $0.013 \pm 0.002$ & $0.287 \pm 0.150$ & $3.52_{-0.46}^{+1.05}$ \\
\hline 3532-WD6 & $28500 \pm 3000$ & $8.5 \pm 0.5$ & $0.96 \pm 0.23$ & $0.060 \pm 0.046$ & $0.240 \pm 0.157$ & $3.73_{-0.57}^{+1.80}$ \\
\hline 3532-WD8 & $23367 \pm 1065$ & $7.71 \pm 0.15$ & $0.48 \pm 0.05$ & $0.023 \pm 0.003$ & $0.277 \pm 0.150$ & $3.56_{-0.47}^{+1.113}$ \\
\hline 3532-WD9 & $29800 \pm 616$ & $7.83 \pm 0.23$ & $0.56 \pm 0.08$ & $0.012 \pm 0.001$ & $0.288 \pm 0.150$ & $3.51_{-0.46}^{+1.04}$ \\
\hline 3532-WD10 & $19267 \pm 974$ & $8.14 \pm 0.27$ & $0.71 \pm 0.11$ & $0.112 \pm 0.030$ & $0.178 \pm 0.150$ & $\begin{array}{l}-0.46 \\
4.14_{-0.78}^{+3.23}\end{array}$ \\
\hline \multicolumn{6}{|l|}{ Praesepe } & \\
\hline WD0836+201 & $16629 \pm 350$ & $8.01 \pm 0.05$ & $0.62 \pm 0.02$ & $0.144 \pm 0.009$ & $0.481 \pm 0.051$ & $2.97_{-0.10}^{+0.11}$ \\
\hline WD0836+199 & $14060 \pm 630$ & $8.34 \pm 0.06$ & $0.82 \pm 0.03$ & $0.39 \pm 0.04$ & $0.235 \pm 0.064$ & $3.76_{-0.28}^{+0.45}$ \\
\hline WD0837+199 & $17098 \pm 350$ & $8.32 \pm 0.05$ & $0.81 \pm 0.03$ & $0.22 \pm 0.02$ & $0.405 \pm 0.054$ & $3.15_{-0.13}^{+0.28}$ \\
\hline WD $0840+200$ & $14178 \pm 350$ & $8.23 \pm 0.05$ & $0.75 \pm 0.02$ & $0.31 \pm 0.02$ & $0.315 \pm 0.054$ & $3.42_{-0.17}^{+0.213}$ \\
\hline WD0836+197 & $21949 \pm 350$ & $8.45 \pm 0.05$ & $0.91 \pm 0.03$ & $0.13 \pm 0.01$ & $0.495 \pm 0.051$ & $2.94_{-0.09}^{+0.11}$ \\
\hline WD0837+185 & $14748 \pm 400$ & $8.24 \pm 0.055$ & $0.76 \pm 0.02$ & $0.288 \pm 0.018$ & $0.337 \pm 0.053$ & $3.35_{-0.16}^{+0.20}$ \\
\hline WD $0837+218$ & $16833 \pm 254$ & $8.39 \pm 0.03$ & $0.85 \pm 0.01$ & $0.251 \pm 0.009$ & $0.374 \pm 0.051$ & $3.23_{-0.13}^{+0.16}$ \\
\hline WD0833+194 & $14999 \pm 233$ & $8.18 \pm 0.035$ & $0.72 \pm 0.01$ & $0.246 \pm 0.009$ & $0.379 \pm 0.051$ & $3.22_{-0.13}^{+0.16}$ \\
\hline WD0840+190 & $14765 \pm 270$ & $8.21 \pm 0.03$ & $0.74 \pm 0.01$ & $0.27 \pm 0.01$ & $0.355 \pm 0.051$ & $3.29_{-0.14}^{+0.17}$ \\
\hline WD0840+205 & $14527 \pm 282$ & $8.24 \pm 0.04$ & $0.76 \pm 0.02$ & $0.30 \pm 0.015$ & $0.325 \pm 0.052$ & $3.39_{-0.16}^{+0.20}$ \\
\hline WD0843+184 & $14498 \pm 202$ & $8.22 \pm 0.04$ & $0.75 \pm 0.02$ & $0.295 \pm 0.012$ & $0.330 \pm 0.051$ & $3.35_{-0.15}^{+0.19}$ \\
\hline \multicolumn{7}{|l|}{ Hyades } \\
\hline WD0352+098 & $14770 \pm 350$ & $8.16 \pm 0.05$ & $0.71 \pm 0.02$ & $0.25 \pm 0.01$ & $0.375 \pm 0.051$ & $3.23_{-0.13}^{+0.16}$ \\
\hline WD0406+169 & $15180 \pm 350$ & $8.30 \pm 0.05$ & $0.79 \pm 0.02$ & $0.29 \pm 0.02$ & $0.335 \pm 0.054$ & $3.35_{-0.16}^{+0.20}$ \\
\hline WD $0421+162$ & $19570 \pm 350$ & $8.09 \pm 0.05$ & $0.68 \pm 0.02$ & $0.096 \pm 0.006$ & $0.529 \pm 0.050$ & $2.88_{-0.09}^{+0.10}$ \\
\hline WD $0425+168$ & $24420 \pm 350$ & $8.11 \pm 0.05$ & $0.70 \pm 0.02$ & $0.038 \pm 0.003$ & $0.587 \pm 0.050$ & $2.78_{-0.08}^{+0.09}$ \\
\hline WD0431+125 & $21340 \pm 350$ & $8.04 \pm 0.05$ & $0.65 \pm 0.02$ & $0.060 \pm 0.004$ & $0.565 \pm 0.050$ & $2.82_{-0.08}^{+0.09}$ \\
\hline WD0438+108 & $27390 \pm 350$ & $8.07 \pm 0.05$ & $0.68 \pm 0.02$ & $0.018 \pm 0.001$ & $0.607 \pm 0.050$ & $2.75_{-0.08}^{+0.08}$ \\
\hline WD0437+138 & $15335 \pm 350$ & $8.26 \pm 0.05$ & $0.77 \pm 0.02$ & $0.26 \pm 0.01$ & $0.365 \pm 0.051$ & $3.26_{-0.14}^{+0.17}$ \\
\hline
\end{tabular}


Table 4.6: Our results based in other available data (continued).

\begin{tabular}{lcccccc}
\hline \hline WD & $\begin{array}{c}T_{\text {eff }} \\
(\mathrm{K})\end{array}$ & $\begin{array}{c}\log g \\
(\mathrm{dex})\end{array}$ & $\begin{array}{c}M_{\mathrm{f}} \\
M_{\odot}\end{array}$ & $\begin{array}{c}t_{\text {cool }} \\
(\mathrm{Gyr})\end{array}$ & $\begin{array}{c}t_{\text {prog }} \\
(\mathrm{Gyr})\end{array}$ & $\begin{array}{c}M_{\mathrm{i}} \\
\left(M_{\odot}\right)\end{array}$ \\
\hline Clusters & & & & & & \\
\hline Pleiades & & & & & & \\
$Z=0.019$ & & & & & & \\
WD0349+247 & $32841 \pm 172$ & $8.63 \pm 0.04$ & $1.03 \pm 0.02$ & $0.048 \pm 0.004$ & $0.071 \pm 0.004$ & $5.87_{-0.13}^{+0.14}$ \\
\hline$Z=0.020$ & & & & & & \\
Sirius B & $25000 \pm 200$ & $8.60 \pm 0.04$ & $1.0 \pm 0.01$ & $0.108 \pm 0.003$ & $0.130 \pm 0.013$ & $4.66_{-0.16}^{+0.19}$ \\
\hline NGC 6791 & & & & & & \\
$Z=0.040$ & & & & & & \\
WD7 & $14800 \pm 300$ & $7.91 \pm 0.06$ & $0.56 \pm 0.02$ & $0.17 \pm 0.01$ & $8.33 \pm 0.01$ & $1.0952_{-0.0003}^{+0.0004}$ \\
WD8 & $18200 \pm 300$ & $7.73 \pm 0.06$ & $0.47 \pm 0.02$ & $0.063 \pm 0.005$ & $8.437 \pm 0.005$ & $1.0936_{-0.0001}^{+0.0002}$ \\
\hline NGC 7789 & & & & & & \\
$Z=0.014$ & & & & & & \\
WD5 & $31200 \pm 200$ & $7.90 \pm 0.05$ & $0.60 \pm 0.02$ & $0.010 \pm 0.0002$ & $1.39 \pm 0.14$ & $1.84_{-0.05}^{+0.07}$ \\
WD8 & $24300 \pm 400$ & $8.00 \pm 0.07$ & $0.63 \pm 0.03$ & $0.029 \pm 0.003$ & $1.37 \pm 0.14$ & $1.85_{-0.05}^{+0.07}$ \\
WD9 & $20900 \pm 700$ & $7.84 \pm 0.12$ & $0.54 \pm 0.04$ & $0.042 \pm 0.006$ & $1.36 \pm 0.14$ & $1.86_{-0.05}^{+0.08}$ \\
\hline NGC 6819 & & & & & & \\
$Z=0.017$ & & & & & & \\
WD6 & $21100 \pm 300$ & $7.83 \pm 0.04$ & $0.54 \pm 0.02$ & $0.041 \pm 0.002$ & $2.46 \pm 0.25$ & $1.57_{-0.05}^{+0.05}$ \\
WD7 & $16000 \pm 200$ & $7.91 \pm 0.04$ & $0.57 \pm 0.01$ & $0.014 \pm 0.005$ & $2.49 \pm 0.25$ & $1.57_{-0.06}^{+0.06}$ \\
\hline \hline
\end{tabular}

by Chen et al. (2003) is $[\mathrm{Fe} / \mathrm{H}]=-0.03$. We use the stellar tracks corresponding to $Z=0.019$.

Sirius B is a very well-known DA white dwarf that belongs to a visual binary system. Since the separation between the members of this system is $\sim 7 \mathrm{AU}$ it can be assumed that there has not been any significant interaction between them during the AGB phase of the progenitor of the white dwarf member (Liebert et al. 2005b). So, we can consider that Sirius B has evolved as a single star, and therefore it is a good candidate for studying the initial-final mass relationship. In this work, we use the atmospheric parameters determined by Liebert et al. (2005b), an age of $237 \pm 12$ Myr and solar metallicity.

NGC 6791, NGC 7789 and NGC 6819 are old open clusters. We include the recent results obtained by Kalirai et al. (2007b) based on spectroscopic observations of white dwarfs in these clusters. These results, as well as those we have obtained using common proper motion pairs constitute the first constraints on the low-mass end of the initial-final mass relationship. NGC 6791 is one of the oldest and most metal-rich open clusters. According to Kalirai et al. (2007a), NGC 6791 has an age 
of $8.5 \mathrm{Gyr}$ and $[\mathrm{Fe} / \mathrm{H}]=+0.3 \pm 0.5$. We have used the stellar tracks corresponding to $Z=0.04$. The white dwarf population of this cluster has relatively low masses $\left(<0.47 M_{\odot}\right)$, so the majority of white dwarfs belonging to this cluster could have a He-core. Although it has been elucidated that He-core white dwarfs could be formed also by single-evolution (Kilic et al. 2007), we prefer not to take these white dwarfs into account, and only consider the two confirmed cluster members with masses above this value and compatible with a $\mathrm{C} / \mathrm{O}$-core composition, as it was done in Kalirai et al. (2007b). The white dwarf population of NGC 7789 and NGC 6819 were also studied recently by Kalirai et al. (2007b). According to their results, NGC 7789 has an age of $1.4 \pm 0.14$ Gyr and a metallicity of $Z=0.014$, while NGC 6819 has an age of $2.5 \pm 0.25 \mathrm{Gyr}$ and $Z=0.017$. We also include these clusters in our analysis. This wealth of observational data is summarized in Table 4.6.

Globular clusters could also be used with the purpose of improving the initial-final mass relationship since they present a scenario similar to open clusters, but containing a higher number of white dwarfs. However, white dwarfs in globular clusters are usually very faint due to their large distances, which difficults the obtention of high $\mathrm{S} / \mathrm{N}$ spectra. Moreover, globular clusters are very crowded, containing from thousands to a million of stars, and it is also difficult to isolate each star properly when performing spectroscopic observations. Therefore, the reduction procedure, mainly the background subtraction, is also more complicated. The white dwarf population of globular clusters have been mainly used to determine their distances and ages. For instance, Richer et al. (2004) studied the white dwarf cooling sequence of M4 to constrain the age of the cluster using photometric observations with HST. Moehler et al. (2004) studied the spectral types and masses of white dwarfs in the globular clusters NGC 6397 and NGC 6752, which in their major part turned out to be of spectral type DA. However, due to the low $\mathrm{S} / \mathrm{N}$ and resolution of the spectra, they were not able to derive their spectroscopic masses independently and had to use a combination of spectra with photometry. Although they calculated an average mass for the white dwarfs in NGC $6752,0.53 \pm 0.02 M_{\odot}$, we have decided not to include this value in our work, since we prefer to use data from independent white dwarfs rather than a binned value for a cluster, on the contrary of other works (Williams 2007; Kalirai et al. 2007b). The use of binned initial mass-final mass points is not realistic since as predicted by the initial mass function of Salpeter, stars of very different masses can be born in the same cluster. Observations of white dwarfs belonging to globular clusters with telescopes of large collective area are essential to improve the determination of their atmospheric parameters, and therefore to derive their masses with accuracy. The further study of white dwarfs in globular clusters will add new points at the low-mass domain of the initial-final mass relationship, since white dwarfs coming from massive progenitors are too faint to be detected. At the same time, these points will correspond to low metallicities, which will be helpful also to evaluate the effect of metallicity on the initial-final mass relationship. 


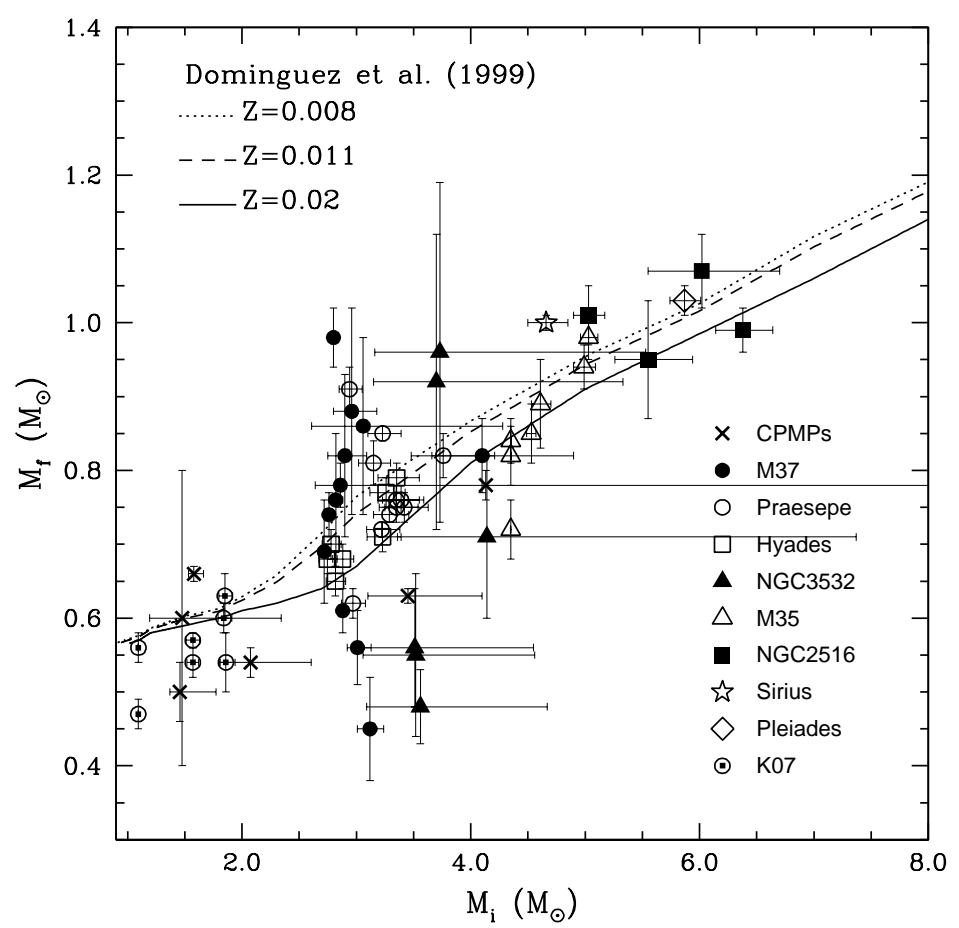

Figure 4.10: Final masses versus initial masses of the available cluster and common proper motion pairs (CPMPs) data. The dotted, dashed and solid lines represent the theoretical initial-final mass relationship of Domínguez et al. (1999) for different metallicities.

\subsection{Discussion}

In Fig. 4.10 we present the final versus the initial masses obtained for white dwarfs in common proper motion pairs and open clusters. The observational data that can be used to define the semi-empirical initial-final mass relationship has now been increased to 62 white dwarfs. It is important to remark that all the values below $2.5 M_{\odot}$ correspond to our data based on common proper motion pairs and the recent data obtained by Kalirai et al. (2007b). Before these studies no data at the low-mass domain were available. This is very important since the coverage of the low-mass end of the initial-final mass relationship guarantees, according to the theory of stellar evolution, the study of white dwarfs with masses near the typical values, $M \sim 0.57 M_{\odot}$, which represent about $90 \%$ of the white dwarf population (Kepler et al. 2007). Thus, with these new data we are increasing the statistical value of the semi-empirical initial-final mass relationship. White dwarfs belonging to stellar clusters are usually more massive, especially if the clusters are young, and thus, less 
representative of the Galactic white dwarf population.

In Fig. 4.10, we have also plotted the theoretical initial-final mass relationships of Domínguez et al. (1999) for different metallicities to be consistent with the stellar tracks used to derive the initial masses. Although there is a large spread, a comparison of the observational data with these theoretical relationships shows that the agreement is fair. The distribution of semi-empirical data presents a considerable dispersion, which is larger than the uncertainties. Moreover, it should be noted that for each cluster the data presents an intrinsic spread in mass. The dispersion varies from cluster to cluster, but it is particularly noticeable for the case of M37. However, the reader should take into account that the observations of M37 were of poorer quality than the rest of the observations (Ferrario et al. 2005). The fact that white dwarf masses in clusters scatter considerably in the same region of initial masses, as pointed out by other authors - see, for instance, Reid (1996) - may indicate that mass loss could depend more on individual stellar properties than on a global mechanism, and that it could be a stochastic phenomenon, especially on the AGB phase. Mass loss has a large impact on the final composition of the outer layers of white dwarfs, since it defines the thicknesses of the He and $\mathrm{H}$ (if present) layers. In fact, another reason that may explain why white dwarfs with such different final masses could have progenitors with very similar initial masses is the simplifications done in the theoretical analysis about the internal compositions and of the outer layer stratification of the white dwarfs under study. As previously pointed out, the thicknesses of the $\mathrm{H}$ and He layers is a key factor in the evolution of white dwarfs, since they control the rate at which white dwarfs cool down. In this work we have used cooling sequences with a given assumption for the thicknesses of these envelopes, which might be more appropriate in some cases than in others. In fact, the exact masses that the layers of $\mathrm{H}$ and He may have is currently a matter of debate. Several authors have studied the effect that the assumption of different thicknesses of the helium and hydrogen layers may have in the cooling times of white dwarfs. For instance, Prada Moroni \& Straniero (2002) computed models reducing the thickness of the $\mathrm{H}$ envelope to $q(\mathrm{H})=2.32 \times 10^{-6}$. Obviously, a different assumption of the amount of $\mathrm{H}$ and $\mathrm{He}$ in the envelopes does not change appreciably the final masses of the white dwarfs, since the mass is mostly concentrated at the core of the star. On the contrary, the cooling times obtained by Prada Moroni \& Straniero (2002) at low luminosities when considering a thinner $\mathrm{H}$ envelope are considerably shorter than those obtained in this work assuming a thick envelope $\left(q(\mathrm{H})=10^{-4}\right)$, since $\mathrm{H}$ has a larger opacity than $\mathrm{He}$, and $\mathrm{H}$ is the major insulating component of the star. In the case of an even thinner $\mathrm{H}$ envelope $\left(q(\mathrm{H})=10^{-10}\right)$ the cooling age could be reduced in 1 Gyr at $\log \left(L / L_{\odot}\right)=-5.5$. Thus, the error in the cooling times could be relevant in some cases, which would affect the progenitor lifetimes and in turn, the initial masses derived. Although this could be a possible source of error, we have not formally introduced this error in the calculations since it is impossible to know which is the real chemical stratification of the outer layers of each individual white 
dwarf, and in some cases we would be overestimating the final error of the initial masses derived.

With the procedure followed to derive the final masses, the final reported errors only take into account the errors in the determination of the atmospheric parameters, which can be derived with accuracy if high signal-to-noise spectra are acquired. It should be taken into account also that this accuracy decreases considerably at low effective temperatures. According to Bergeron et al. (1992), atmospheres of DA stars below $12000 \mathrm{~K}$ could well be enriched in helium, while retaining their DA spectral type. This helium is brought to the surface when a significant hydrogen convection zone develops. Depending on the efficiency of convection the star could preserve the appearance of the Balmer lines, instead of turning into a non-DA white dwarf. Thus, the assumption of an unrealistic chemical compositon could have a larger impact on the cooling times estimated, but mainly at the low effective temperatures. Nevertheles, a very large fraction $(\sim 95 \%)$ of the stars in our sample have temperatures well above this limit.

On the other hand, the procedure used to derive the initial masses involves more parameters and models. For instance, it depends on the cooling times, the total ages, the metallicity and finally, on the stellar tracks used. Among these parameters the largest source of error is due to the uncertainty in the total ages of the white dwarfs. For white dwarfs in open clusters, the error in the age can be usually derived with high accuracy from model fits to the turn-off location in a color-magnitude diagram. The effect of the error in the age in a sample of white dwarfs belonging to the same cluster is systematic, since all the initial masses will be shifted together to larger or smaller masses in the final versus initial masses diagram. On the contrary, in the case of white dwarfs in common proper motion pairs, the accuracy in the total age depends on the evolutionary stage of the companion.

In this work we are presenting a complete semi-empirical initial-final mass relationship. Thus, a detailed analysis of these results can allow us to give some clues on the dependence of this relationship with different parameters (introduced in chapter $1)$ :

- Metallicity: The sample of white dwarfs studied here covers a range of metallicities from $Z=0.006$ to 0.040 . From a theoretical point of view it is well established that progenitors with large metallicity produce less massive white dwarfs, because mass-loss mechanisms depend on metallicity - see the relations of Domínguez et al. (1999) also plotted in Fig. 4.10. Thus, one should expect to see a dependence of the semi-empirical data with metallicity. As pointed out in chapter 2 , when data of the same metallicity are considered, it turns out that for equal progenitor masses very different final masses can be obtained. Open clusters present an appropriate scenario for carrying out such comparison, since all the stars belonging to a particular cluster have the same metallicity. If the data of the two stars from Praesepe (open circles) that have 
an initial mass of $3.0 M_{\odot}$ are compared, it can be noted that the scatter in the final masses obtained is $\Delta M_{\mathrm{f}}=0.3 M_{\odot}$. However, for the rest of stars in this cluster, the dispersion is significantly smaller (a factor of 2). Thus, the large spread could be explained by considering that maybe these two objects are field stars. However, the sample of white dwarfs in the Praesepe cluster has been well studied (Claver et al. 2001; Dobbie et al. 2004) and it is unlikely that these stars do not belong to the cluster. The Hyades contain a large number of white dwarfs, for which the initial and final masses have been derived with accuracy. In this case, all the points fall in the region limited by the theoretical relations of Domínguez et al. (1999), and the scatter is $\Delta M_{\mathrm{f}}=0.1 M_{\odot}$, which is the same as the difference between the theoretical relations corresponding to $Z=0.008$ and $Z=0.02$. This is the minimum scatter found in the observational data, since the points corresponding to the Hyades, together with those of Praesepe, are the ones with smaller error bars. In any case, this scatter, although smaller than in other cases, is still larger than the uncertainties, which prevents to see any clear dependence on metallicity. As previously pointed out, the data of M37 presents the largest scatter in the sample $\left(\Delta M_{\mathrm{f}}=0.5 M_{\odot}\right)$, but it should be noticed that the errors in the final masses are considerably larger than for the rest of the clusters.

It is important to evaluate if the dispersion increases when data with different metallicities are considered. Comparing points with the same initial mass but different metallicities, it can be noted how $\Delta M_{\mathrm{f}}$ is of the same order as in the case of equal metallicities. For example, comparing the data from M37 and the data of the Hyades - with $Z=0.011$ and $Z=0.027$, respectively - for an initial mass around $3 M_{\odot}, \Delta M_{\mathrm{f}}$ is of the order of $0.5 M_{\odot}$, but this scatter has the same value when we compare only data from M37. However, the scatter decreases when we go to larger initial masses. If we compare the data of M35 and M37 (with $Z=0.011$ ) and the data from NGC3532 and one of the common proper motion pairs $(Z=0.02)$ located at $\sim 4.0 M_{\odot}$, it can be seen that the maximum $\Delta M_{\mathrm{f}}$ is $0.2 M_{\odot}$. But this scatter is the same if we compare only the M35 data located at this region. In the region of small masses we have points ranging from $Z=0.008$ to $Z=0.04$, but the maximum scatter is the same regardless of the metallicity of the stars $\left(\Delta M_{f}=0.1 M_{\odot}\right)$. Finally, at the high-mass end of the semi-empirical data we find the same dispersion, although in this case the data has the same metallicity. Thus, considering the current accuracy of the available observational data we do not note any clear dependence of the semi-empirical initial-final mass relationship with metallicity.

Following closely the works of Ferrario et al. (2005) and Williams (2007), we assume that the initial-final mass relationship can be described as a linear function. Since we have not found a clear dependence with metallicity, there 


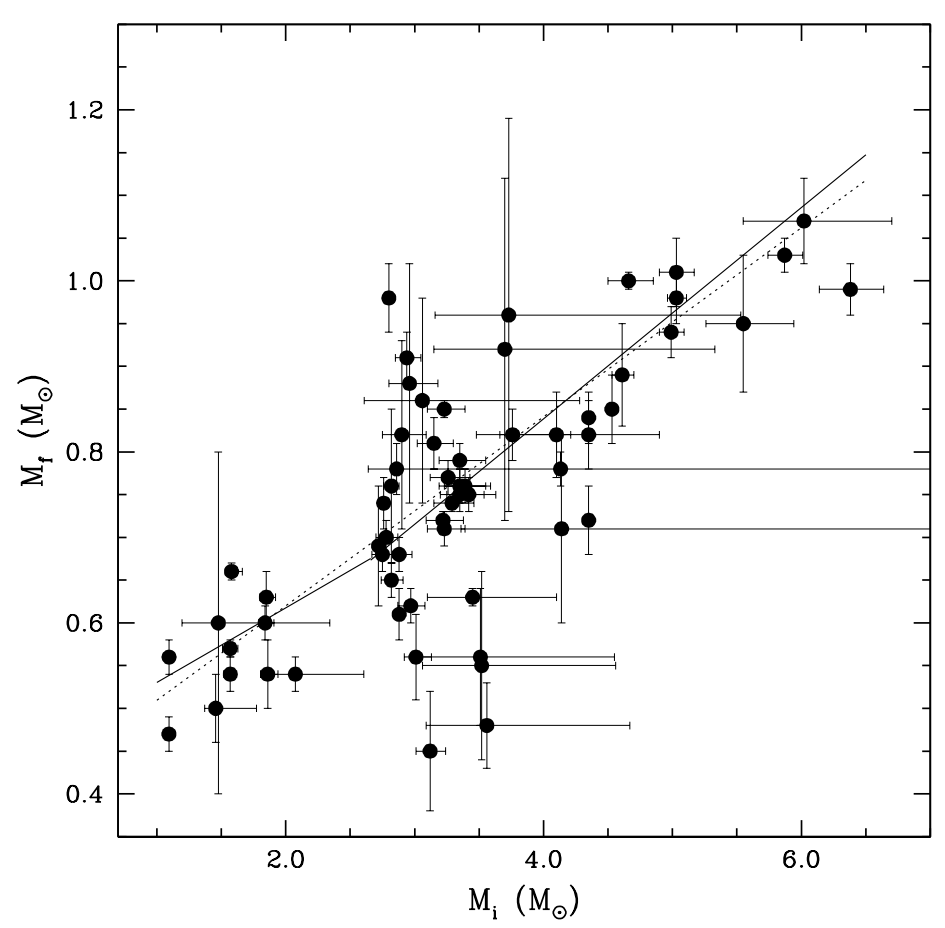

Figure 4.11: Final masses versus initial masses of the available cluster and common proper motion pair data. Solid and dotted lines correspond to linear least-squares fits of the data.

is no compelling reason to justify the use of a more sophisticated relationship. We have performed a weighted linear least-squares fit of these data, obtaining that the best solution is

$$
M_{\mathrm{f}}=(0.111 \pm 0.003) M_{\mathrm{i}}+(0.399 \pm 0.009)
$$

where the errors are the standard deviation of the coefficients.

In Fig. 4.11 we represent all the data that we have re-calculated and this linear fit (dotted line). As can be seen from Fig. 4.10 the theoretical initial-final relationship could be divided in two different linear functions, each one above and below $2.7 M_{\odot}$, becoming approximately flat at small masses due to the less efficient mass-loss processes. Considering this change in the slope of the initialfinal mass relationship, we have performed a weighted linear least-squares fit for each region, obtaining: 


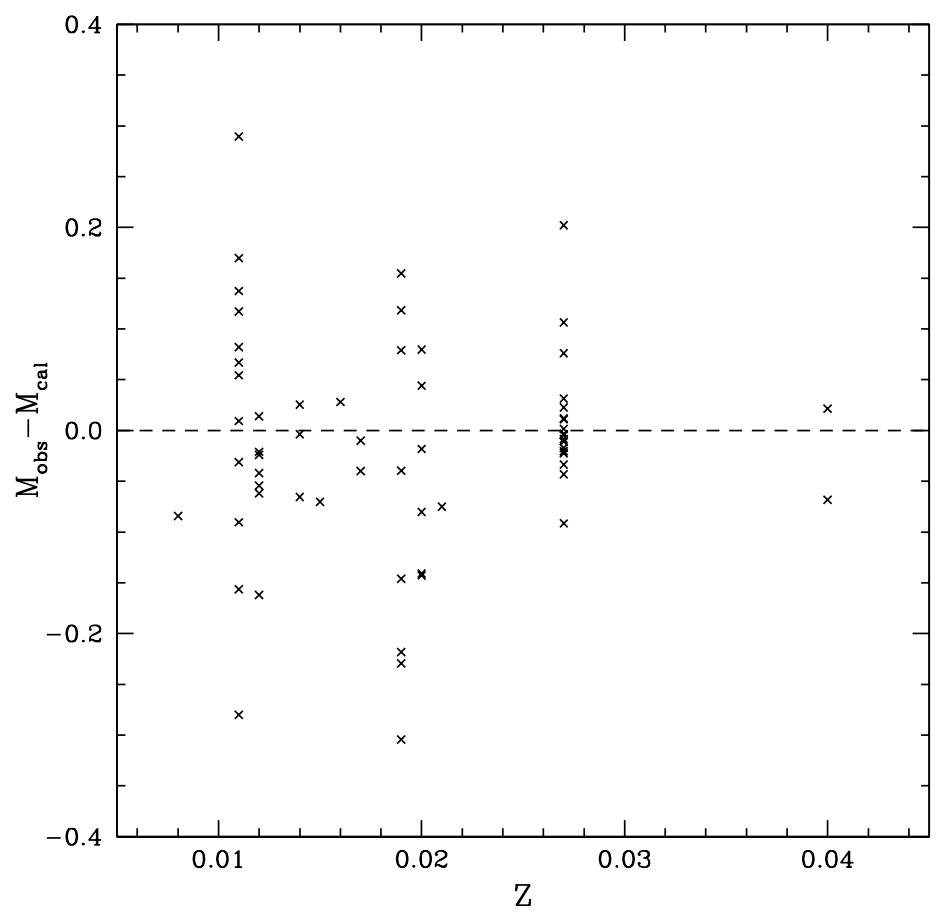

Figure 4.12: Correlation between final masses and metallicity.

$$
M_{\mathrm{f}}= \begin{cases}(0.087 \pm 0.005) M_{\mathrm{i}}+(0.443 \pm 0.014) & \text { for } \quad M_{\mathrm{i}}<2.7 M_{\odot} \\ (0.123 \pm 0.005) M_{\mathrm{i}}+(0.344 \pm 0.019) & \text { for } \quad M_{\mathrm{i}}>2.7 M_{\odot}\end{cases}
$$

where the errors are the standard deviation of the coefficients. These two independent fits, which are represented as solid lines in Fig. 4.11, seem to reproduce better the observational data than a unique linear fit (dotted line). Taking into account the scatter of the data and the reduced $\chi^{2}$ of these fits, which are 5.4 and 8.3, respectively, we consider that the errors associated to the coefficients are underestimated. A more realistic error can be obtained if we calculate the dispersion on the derived final masses, which is of $0.05 M_{\odot}$ and $0.14 M_{\odot}$ respectively. These are the errors that should be associated to the final mass when using the expressions derived here.

It is interesting to perform a quantitative study of the correlation between the final masses and the metallicity. In Fig. 4.12 we plot the differences between the observed final masses and the final masses obtained using Eq. 4.2 as a function 
of metallicity. The dashed line corresponds to the hypothetical case in which there is no difference between the observational and the derived values. In order to quantify the correlation in the sample of points presented in Fig. 4.12, we have calculated the Spearman rank correlation coefficient. The value obtained is 0.028 , which is very close to zero, indicating that there is an extremely weak positive correlation between the difference in the final masses and metallicity. Since the Spearman correlation does not take into account the errors, we have carried out a bootstrapping in order to evaluate the actual uncertainty on the correlation coefficient without any assumption on the error bars. This consists on choosing at random $N$ objects from our sample (which has also $N$ objects) allowing repetition, and then calculating the new correlation coefficient for each of these new samples. We have performed this a high number of times obtaining a mean correlation coefficient of $0.002 \pm 0.128$. Thus, we can conclude that the final masses and metallicity of this sample do not present any correlation, and that the scatter in the distribution in Fig. 4.10 is not due to the effect of metallicity. Of course, the reader should take into account that the observed final masses have been derived by using the atmospheric parameters reported by different authors who have considered also different white dwarf models, although the prescriptions used in the fits are usually those of Bergeron et al. (1992) in all the cases.

At this point of the discussion, it is worth to compare our results with other works. Kalirai et al. (2005) suggested that they had found the first evidence of a metallicity dependence on the initial-final mass relationship. They noticed that half of their data of M37 (also plotted in Fig. 4.10) were in agreement with the theoretical relationship of Marigo (2001), and considered this result as an indication of dependence on metallicity. However, this dependence does not seem very clear since there is not any reason to consider that the rest of the data of this cluster correspond to field stars, and also, these data is somewhat of lower quality because the stars were much fainter than in other studies (Ferrario et al. 2005). On the other hand, in a recent revision of the semi-empirical initial-final mass relationship, Williams (2007) analyzed part of the cluster data discussed here by deriving a binned semi-empirical initial-final mass relationship. This consisted in associating a initial and final mass for each cluster by calculating the mean of the initial and final masses of the individual white dwarfs belonging to that cluster. Then, they compared these values as function of metallicity and, as in our case, did not find a clear dependence of the semi-empirical data with metallicity. In fact, their conclusion was that for a star with an initial mass of $3 M_{\odot}$, metallicity should affect the final mass only in $0.05 M_{\odot}$, which is in good accord with our results.

Thus, we conclude that the effect of metallicity is not reflected in the semiempirical data obtained, or at least when due account of the error bars is 
taken this effect seems to be very weak. The origin of the large scatter in the semi-empirical data might lie on the different assumptions considered during the analysis of the data (cooling sequences, stellar tracks, age and metallicity errors...), as well as other parameters that may have an important influence on the evolution of a star (rotation, magnetism...). However, even if we have not been able to detect a clear dependence on metallicity it is convenient to point out that it might have an influence on the final mass according to current theories of stellar evolution (Domínguez et al. 1999; Marigo 2001; Hurley et al. 2000), but the magnitude of the effect still remains to be assessed.

- Rotation: according to Domínguez et al. (1996), if a main-sequence star is a fast rotator it produces a more massive white dwarf than if it is a slow rotator. The models calculated by Domínguez et al. (1996) including rotation predict that, for a fast rotator star with an initial mass of $6.5 M_{\odot}$, the white dwarf produced has a mass ranging from 1.1 to $1.4 M_{\odot}$, considerably larger than those obtained when rotation is disregarded (Domínguez et al. 1999). Among our sample of white dwarfs in common proper motion pairs there are two stars (WD1659-531 and WD1620-391) that might exemplify the effect that rotation may have in stellar evolution. The companion of WD1659-531, HD153580 is a fast rotator star according to Reiners \& Schmitt (2003), with a tangential velocity $v \sin i=46 \pm 5 \mathrm{~km} / \mathrm{s}$. It is therefore reasonable to assume that the progenitor of WD1659-531 was also a fast rotator. Starting from an initial mass of $1.58 M_{\odot}$, which is more than two times smaller than the progenitor of WD1620-391, it ends up as a white dwarf with approximately the same mass, $0.64 M_{\odot}$. This indicates that the progenitor of WD1659-531 lost less mass during the AGB phase than the progenitor of WD1620-391. These differences may not be related to metallicity, since both progenitors had a solar composition. Thus, we think that this could be the first evidence showing that rotation may have a strong impact in the evolution of a star, leading to more massive white dwarfs, as suggested by Domínguez et al. (1996).

- Magnetism: as explained in chapter 1, one way to detect the presence of magnetic fields in white dwarfs is by performing spectropolarimetric observations. Some of the white dwarfs in our sample have been the subject of these kind of studies to confirm or discard their magnetic nature. Kawka et al. (2007) obtained circularly polarized spectra of WD0413-017, WD1544-377, WD1620-391 and WD1659-531, finding evidence of magnetism only for the first of these stars. This result is in good agreement with the findings of other authors (Fabrika et al. 2000; Aznar Cuadrado et al. 2004; Jordan et al. 2007). The typical mass of magnetic white dwarfs is around $0.9 M_{\odot}$. However, as detailed in chapter 2 the mass of WD0413-017 is $0.54 \pm 0.02 M_{\odot}$, which is nearly half of this typical value. Regarding its progenitor, it is massive in comparison with the progenitor masses obtained for white dwarfs with the same 


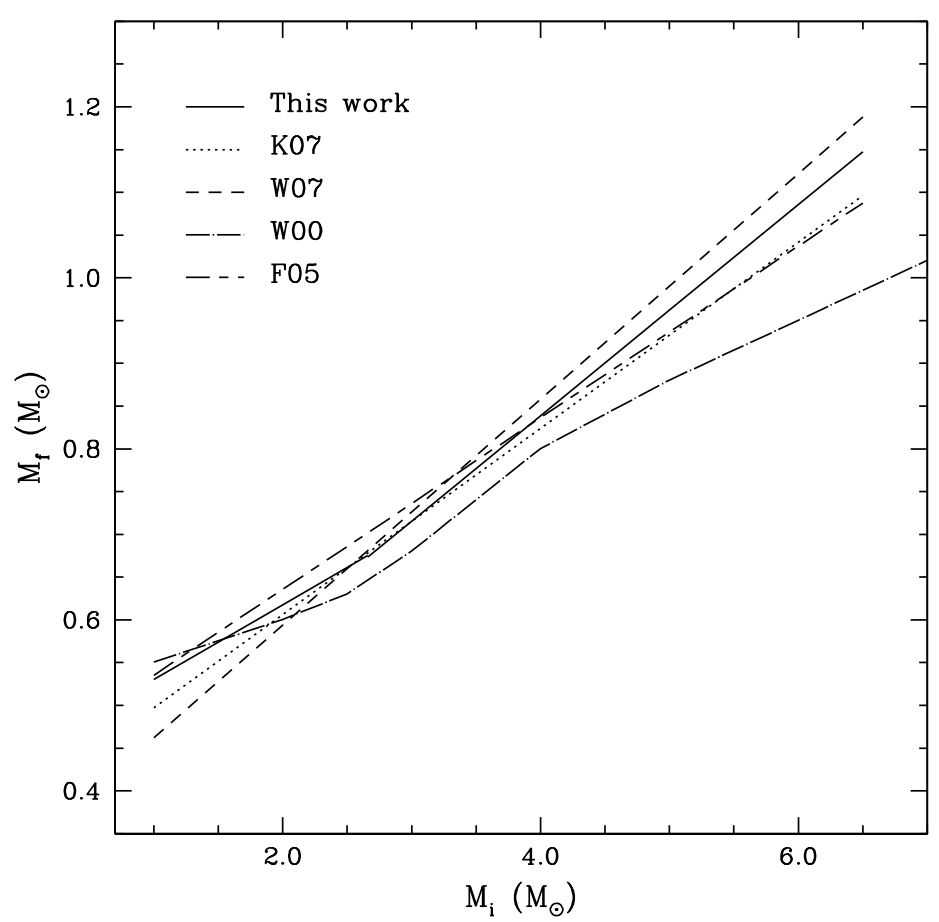

Figure 4.13: Comparison of our semi-empirical initial-final mass relationship and those of different authors: Kalirai et al. (2007b) - K07 — Williams (2007) - W07 - Weidemann (2000) - W00 and Ferrario et al. (2005) - F05.

mass as WD0413-017. Taking this into consideration, it cannot conclusively be obtained any positive answer about the effect that magnetic fields may have in stellar evolution. The identification of the magnetic white dwarfs that may exist among this sample, and the corresponding spectropolarimetric observations to quantify the strength of the magnetic fields, if present, are needed in order to be able to evaluate the effects of magnetism in the initial-final mass relationship in a more comprehensive manner.

\subsection{Final remarks}

It is important to realize that thanks to the analysis carried out in this work using common proper motion pairs and that of Kalirai et al. (2007b) using clusters, the semi-empirical initial-final mass relationship has been now extended to the region of low-mass stars. Thus, there is no need to use a ficticious anchor point in our fit at 
low masses as in previous works (Ferrario et al. 2005), since now we are reproducing the well-established peak in the field white dwarf mass distribution at $M_{\mathrm{f}} \sim 0.57 M_{\odot}$ (Kepler et al. 2007).

Finally, the semi-empirical initial-final mass relationship obtained here can be compared with those obtained previously. For instance, a comparison can be done with the relationship of Weidemann (2000), and the most recent ones obtained by Ferrario et al. (2005), Kalirai et al. (2007b) and Williams (2007). This is done in Fig. 4.13, and as can be observed our relation is in good agreement with the most recent ones, favoring the production of massive white dwarfs. In the region of small masses though, our fit is in better agreement with the relation of Weidemann (2000), being less steep. This can be explained considering that we have divided the observational data in two different regions when performing the linear least-squares fits. 


\section{Chapter 5}

\section{The white dwarf luminosity function}

\section{$5.1 \quad$ Introduction}

In this chapter we compute the white dwarf luminosity function. This issue is important because a comparison of the theoretical white dwarf luminosity function and the observational data available can provide us with important information about both the star formation history (Noh \& Scalo 1990; Díaz-Pinto et al. 1994) and the age of the Galaxy (Winget et al. 1987; García-Berro et al. 1988), and because it depends on the initial-final mass relationship of white dwarfs, which is the main subject of this work.

One of the key issues in computing theoretical white dwarf luminosity functions is not only taking into account the latest advances in the theory of cooling white dwarfs (for which we now have an accurate description until moderately low luminosities) but also to properly take into account the influence of the progenitors. As it will be seen below, among the inputs required to compute the white dwarf luminosity function, the progenitor lifetime and the initial-final mass relationship turn out to be essential ingredients. Based on very basic theoretical grounds it is expected that both the progenitor lifetime and the initial-final mass relationship depend on metallicity. Although from the semi-empirical results obtained in chapters 2 and 4 there is no clear evidence of the dependence of the latter with metallicity, there are compelling theoretical reasons - see chapter 1 - to suspect that such dependence indeed exists. Along this chapter we will explore the behavior of the white dwarf luminosity function on these essential stellar evolutionary inputs.

We start by reviewing the most basic concepts. We recall that the white dwarf luminosity function is defined as the number of white dwarfs per unit volume and per bolometric magnitude - see, for instance, Isern et al. (1998): 


$$
n\left(M_{\mathrm{bol}}, T\right)=\int_{M_{\mathrm{i}}}^{M_{\mathrm{s}}} \phi(M) \psi\left(T-t_{\mathrm{cool}}-t_{\mathrm{prog}}\right) \tau_{\mathrm{cool}} d M
$$

where $M$ is the mass of the progenitor of the white dwarf, $\tau_{\text {cool }}=d t / d M_{\text {bol }}$ is its characteristic cooling time, $M_{\mathrm{i}}$ and $M_{\mathrm{s}}$ are the minimum and maximum mass of the progenitor star able to produce a white dwarf with a bolometric magnitude $M_{\text {bol }}$ at time $T, t_{\text {cool }}$ is the time necessary to cool down to bolometric magnitude $M_{\text {bol }}$ for which we adopt the results of Salaris et al. (2000) $-t_{\text {prog }}$ is the lifetime of the progenitor, $T$ is the age of the population under study, $\psi(t)$ is the star formation rate - which we assume to be constant - and $\phi(M)$ is the initial mass function, for which we adopt the expression of Salpeter (1955):

$$
\phi(M)=0.225 M^{-2.35}
$$

\subsection{The influence of the progenitors}

As can be seen from Eq. (5.1), in order to compute the white dwarf luminosity function it is also necessary to provide a relationship between the mass of the progenitor and the mass of the resulting white dwarf, i. e., the initial-final mass relationship. Additionally, the influence of the progenitors in Eq. (5.1) appears through the age assigned to the progenitor, which determines the star formation rate, and through the cooling time, $t_{\text {cool }}$ and the characteristic cooling time, $\tau_{\text {cool }}$, which depend on the mass of the white dwarf. In order to evaluate the influence of these inputs, we have computed a series of theoretical white dwarf luminosity functions using several initial-final mass relationships (see Fig. 5.1) and evolutive tracks for the progenitor stars (see Fig. 5.2). Whenever possible we have adopted the same set of stellar evolutionary inputs. That is, we have adopted the initial-final mass relationship and the main-sequence lifetime corresponding to the same set of calculations.

We compared the resulting theoretical luminosity functions with the data obtained by averaging the different observational determinations of the white dwarf luminosity function using the $1 / V_{\max }$ method (Schmidt 1975). When using this method, each star is weighted by the inverse of the maximum volume $\left(V_{\max }\right)$ within which that same star hypothetically could be found, given the presumed completeness of the sample in both proper motion and apparent magnitude. The resulting white dwarf luminosity functions were normalized to the observational value with the smallest error bars in density, that is $\log (N)=-3.610, \log \left(L / L_{\odot}\right)=-2.759$, avoiding in this way the region in which the cooling is dominated by neutrinos (at higher luminosities) and the region in which crystallization is the dominant physical process - which is located between $\log \left(L / L_{\odot}\right) \simeq-3$ and -4 .

Fig. 5.3 shows the resulting white dwarf luminosity function when the stellar evolutionary inputs of Domínguez et al. (1999) are used for several ages of the galactic 


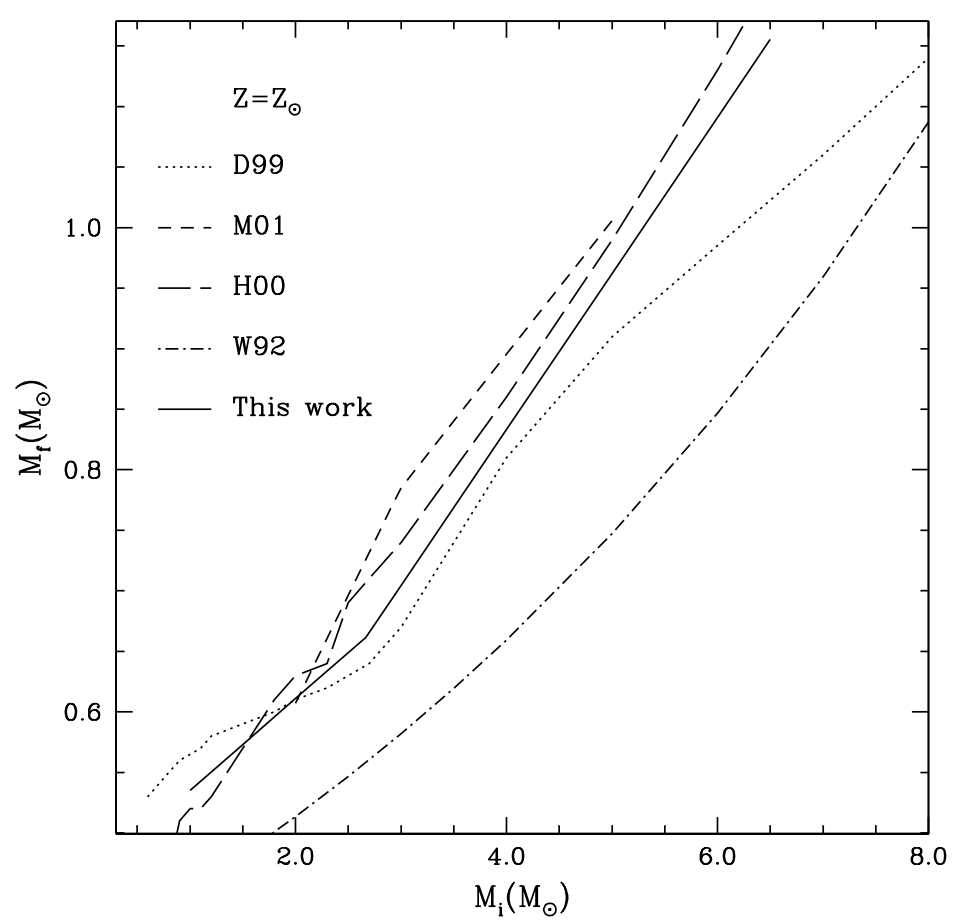

Figure 5.1: Initial-final mass relationship according to different authors: Domínguez et al. (1999) — D99 — Marigo (2001) - M01 - Hurley et al. (2000) - H00 — Wood (1992) W92 - and the one derived in this work.

disk. At low luminosities we can notice a sharp down-turn of the density of white dwarfs. This cut-off in the number counts has been interpreted by different authors (Winget et al. 1987; García-Berro et al. 1988) as the consequence of the finite age of the Galactic disk. Thus, a comparison between the theoretical luminosity functions and the observational data can provide information about the age of the Galactic disk. As can be seen, the disk age that best fits the data is $T=11 \mathrm{Gyr}$. We can perform the same calculations but considering the initial-final mass relationship that we have derived in the last chapter using the available data and our results based in common proper motion pairs (Catalán et al. 2008a). From Fig. 5.1 it can be noted that this semi-empirical initial-final mass relationship is in good agreement with the relation of Domínguez et al. (1999) for small masses, although at large masses it is in better agreement with that of Hurley et al. (2000), favoring the production of massive white dwarfs. In Fig. 5.4 we show the theoretical luminosity functions obtained when considering this new semi-empirical initial-final mass relationship. As can be seen, the best fit to the observational data, in particular to the position of the cut-off of the white dwarf luminosity function, is also obtained when $T=11$ Gyr. 


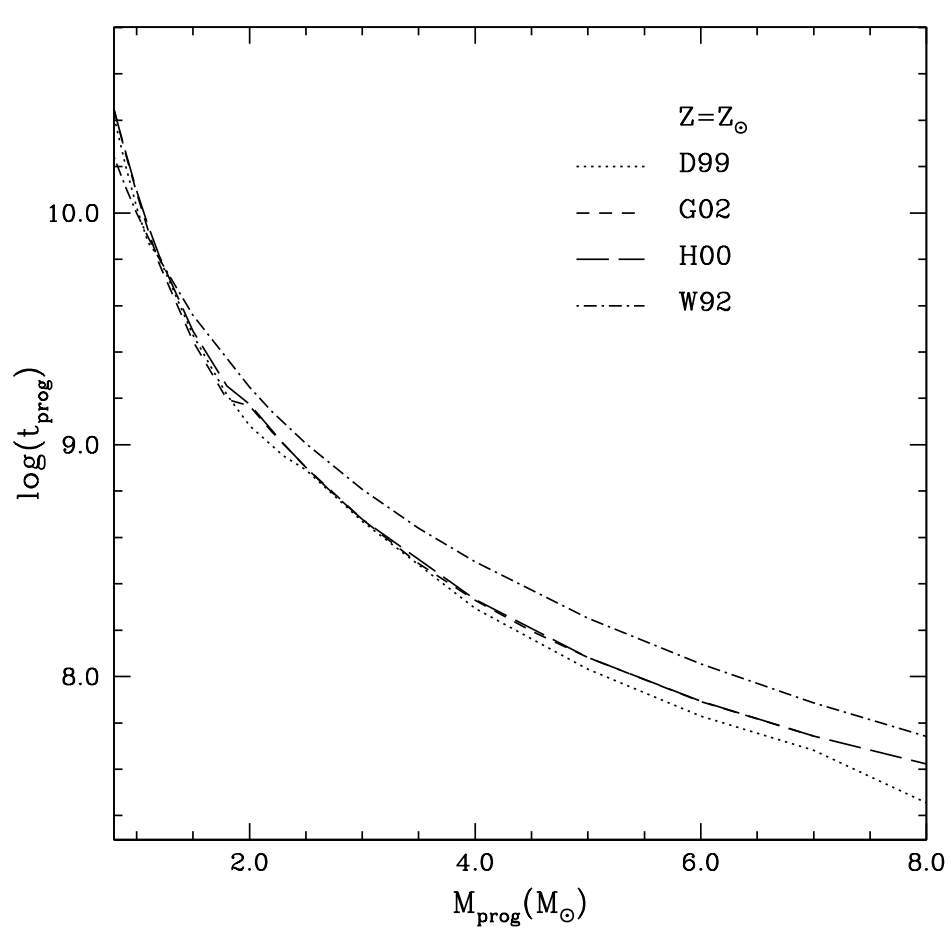

Figure 5.2: Main sequence lifetime versus stellar mass according to different authors: Domínguez et al. (1999) — D99 — Girardi et al. (2002) — G02 - Hurley et al. (2000) - H00 — and Wood (1992) — W92.

Similar results are obtained when using the stellar evolutionary inputs of Girardi et al. (2002) and the analytical models of Hurley et al. (2000), but we do not show them graphically for the sake of conciseness.

On the contrary, if the main-sequence lifetimes and the initial-final mass relationship proposed by Wood (1992) — largely used in the literature — given by:

$$
t_{\text {prog }}=10\left(\frac{M_{\text {prog }}}{M_{\odot}}\right)^{-2.5}
$$

and

$$
M_{\mathrm{WD}}=0.40 e^{0.125 M_{\mathrm{prog}}}
$$

are employed, the age of the disk that best fits the observational data corresponds to a younger age with respect to that obtained in the rest of the cases, $T=10.5 \mathrm{Gyr}$ (Fig. 5.5). This can be understood by comparing the different stellar evolutionary inputs considered in this work. As can be seen in Fig. 5.1, the initial-final mass relationship of Wood (1992) is the one that produces less massive white dwarfs. The 


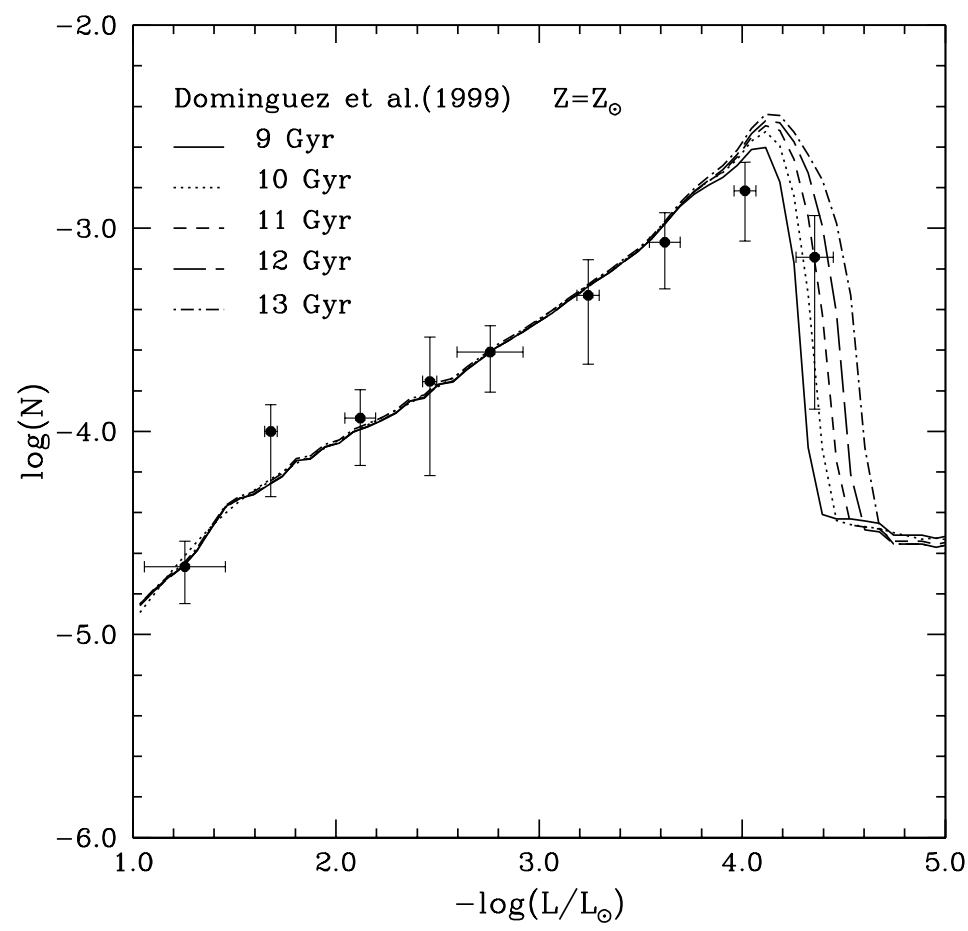

Figure 5.3: White dwarf luminosity function considering the stellar evolutionary inputs of Domínguez et al. (1999) for different ages: 9, 10,11, 12 and 13 Gyr. The best fit is obtained for $T=11 \mathrm{Gyr}$.

calculations of Marigo (2001) and Hurley et al. (2000) obtain similar values for the high mass end, whereas the results of Domínguez et al. (1999) produce more massive remnants for the low-mass end and less massive white dwarfs for the high mass end, but always substantially larger than those obtained with the initial-final mass relationship of Wood (1992). These differences mainly arise from the procedure used to calculate each theoretical initial-final mass relationship. While the relations of Wood (1992) and Hurley et al. (2000) are analytic, the ones derived by Domínguez et al. (1999) and Marigo (2001) come from computing fully evolutive models but using different treatments of convective boundaries, mixing and mass-loss rates on the AGB phase - see appendix B. In Fig. 5.2 we show the corresponding different main-sequence lifetimes as a function of the main-sequence mass. In those cases in which there was a dependence with metallicity we have adopted $Z=Z_{\odot}$. As it can be seen there, the behavior of the different main-sequence lifetimes is very similar, although for the case of Wood (1992), stars spend more time in the main-sequence than in the rest of cases, especially for those stars with large masses. Considering this, one should expect that the fit of the cut-off of the white dwarf luminosity function 


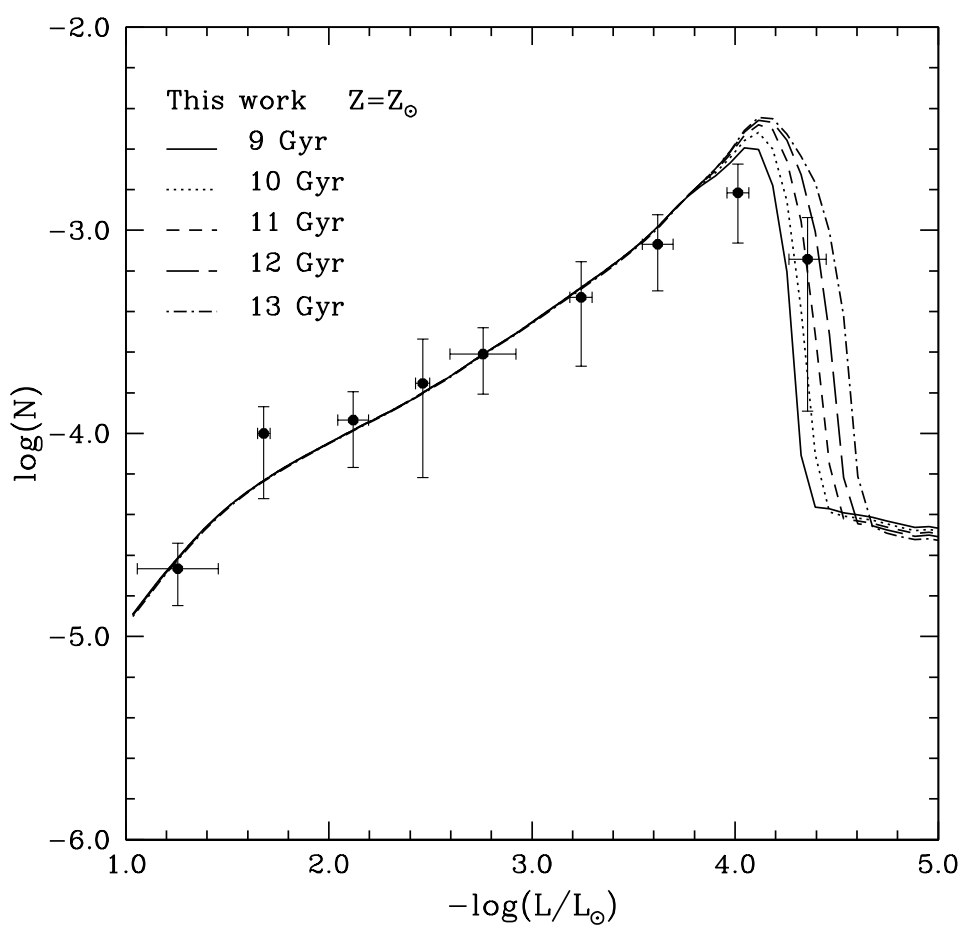

Figure 5.4: White dwarf luminosity function for different disk ages ranging from 9 to $13 \mathrm{Gyr}$, using the stellar tracks of Domínguez et al. (1999) and the semi-empirical initial-final mass relationship obtained in chapter 3 . The best fit is obtained for $T=11$ Gyr.

will correspond to a longer Galactic disk age. On the contrary, we have obtained a younger Galactic disk. The longer progenitors lifetimes of Wood (1992) are in part compensated by the fact that its corresponding initial-final mass relationship favors the production of low-mass white dwarfs, which cool down faster at high luminosities. A simple test can be done by using, for example, the stellar tracks of Domínguez et al. (1999), which give shorter progenitor lifetimes, and the initial-final mass relationship of Wood (1992). In this case we obtain a Galactic disk age of 10 Gyr, 1 Gyr younger than if we consider any of the other initial-final mass relationships showed in Fig. 5.1. Thus, the behavior of the relation of Wood (1992) is clearly different from others in the literature, and this has important implications on the resulting white dwarf luminosity functions.

It is as well interesting to compare the different luminosity functions obtained so far when the cut-off of the observational white dwarf luminosity function is fitted. This is done in Fig. 5.6. It can be noticed that for the hot branch of the white dwarf luminosity function there are not differences whatsoever. In fact, all the theoretical luminosity functions are remarkably coincident. The only visible dif- 


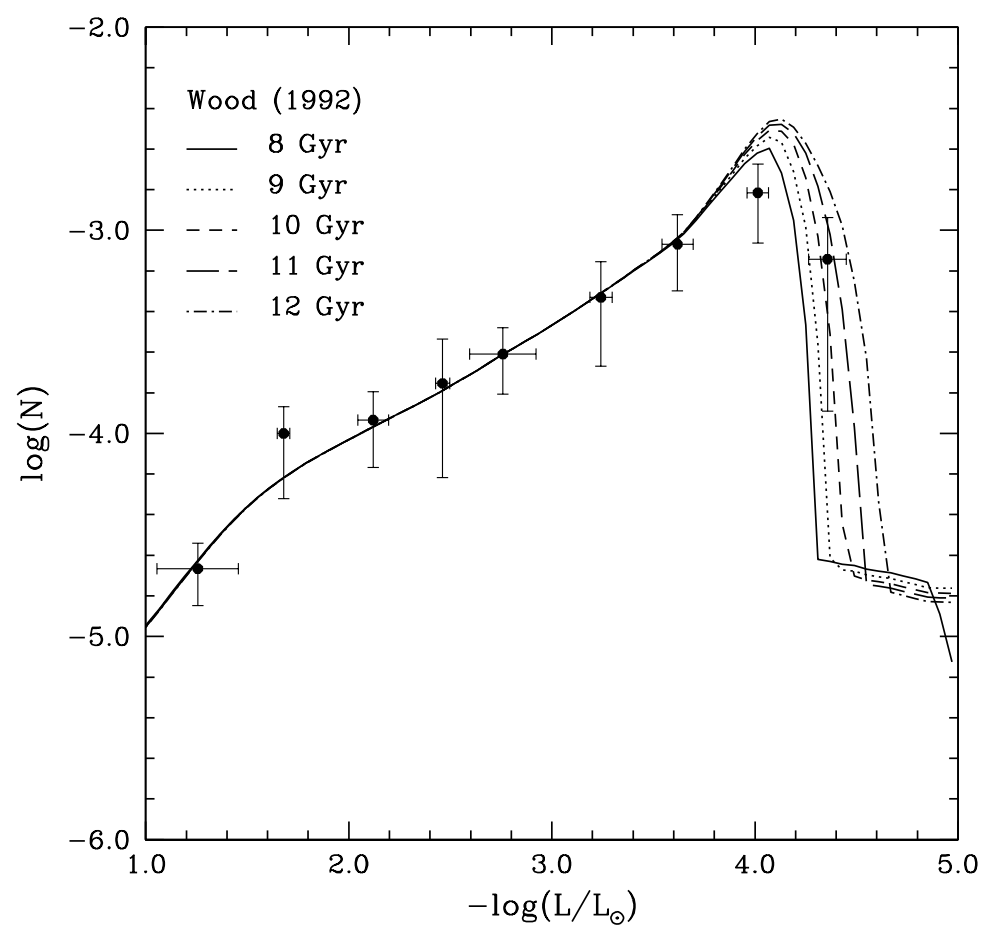

Figure 5.5: White dwarf luminosity function for different disk ages ranging from 8 to $12 \mathrm{Gyr}$, when the expressions of Wood (1992) are used. The best fit is obtained for $T=10.5$ Gyr.

ferences, although not very relevant, occur just after the crystallization phase has started, at $\log \left(L / L_{\odot}\right) \simeq-4.0$. Two essential physical processes are associated with crystallization, namely, a release of latent heat and a modification of the chemical concentrations in the mixture, which both provide extra energy sources and lengthen the cooling time of the star. This is the reason why all the theoretical calculations predict a larger number of white dwarfs for these luminosity bins. Beyond the cutoff, we find that the density of white dwarfs is smaller in the case of Wood (1992), and this is because this region is dominated by massive white dwarfs, since the progenitors of these stars were also massive, and as shown in Fig. 5.2 spent less time at the main-sequence. Thus, massive white dwarfs have had enough time to cool down to such low luminosities. In any case, it is worth mentioning that low-mass white dwarfs cool faster at high luminosities, but for small luminosities it occurs just the opposite, since massive white dwarfs crystallize at larger luminosities, and this implies smaller time delays.

The remarkable unicity of the hot branch of the white dwarf luminosity function can be easily understood taking into account that "hot" means small values of $t_{\text {cool }}$ in Eq. (5.1) and that stars born at very different epochs of the life of the Galaxy can 


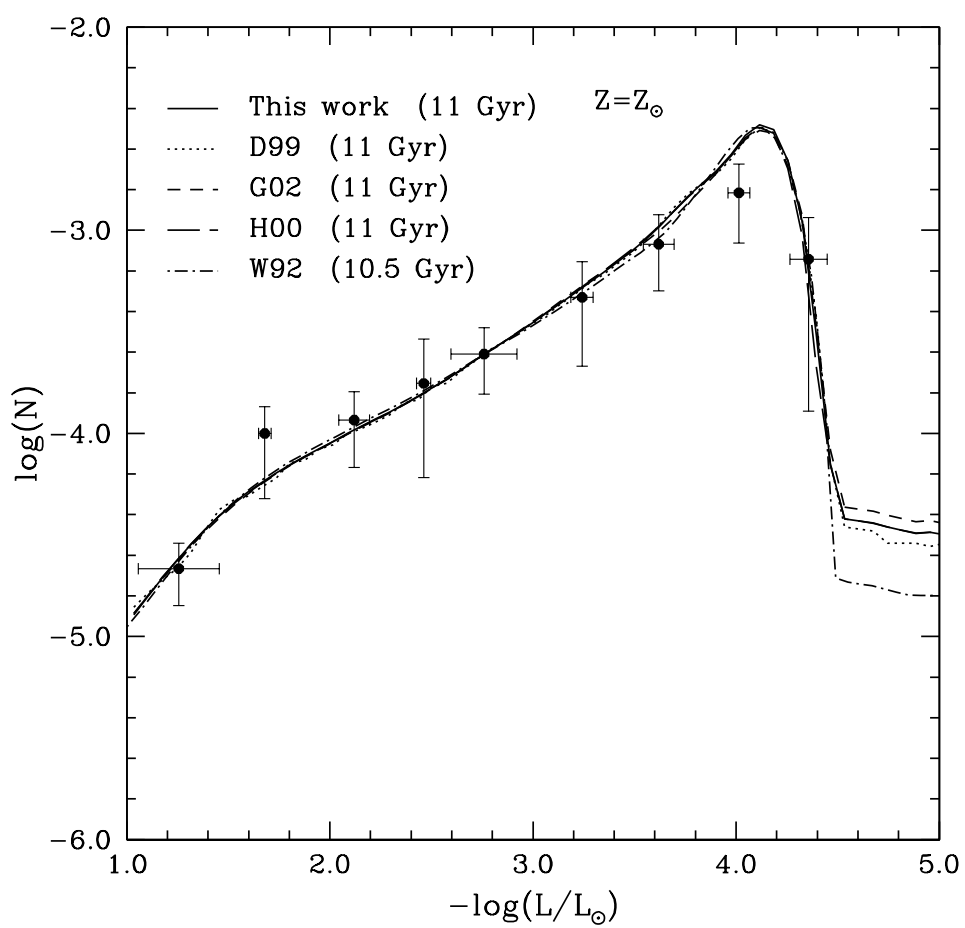

Figure 5.6: White dwarf luminosity functions considering different evolutive stellar models and initial-final mass relationships: Domínguez et al. (1999) - D99 - Girardi et al. (2002) — G02 - Hurley et al. (2000) - H00 - Wood (1992) - W92 - and the relation derived in this work. See text for details.

contribute to this luminosity range of the luminosity function. Since $\tau_{\text {cool }}$ is weakly dependent on the mass of the white dwarf, if the star formation rate is constant or nearly constant, any difference is erased by the process of normalization and the shape of the luminosity function is completely determined by the characteristic cooling time. Notice as well that near the cut-off this is no longer valid, since $t_{\text {cool }} \sim T$, and the function is sensitive to the different hypothesis that have been introduced (Isern et al. 1998).

\subsection{The luminosity function of massive white dwarfs}

The influence of the initial-final mass relationship on the white dwarf luminosity function should be more evident when it is constrained to massive white dwarfs since the fraction of massive white dwarfs is in principle dominated by this relationship. In Fig. 5.7 we plot the luminosity functions that we have computed using different initial-final mass relationships (and their corresponding stellar tracks). For such 


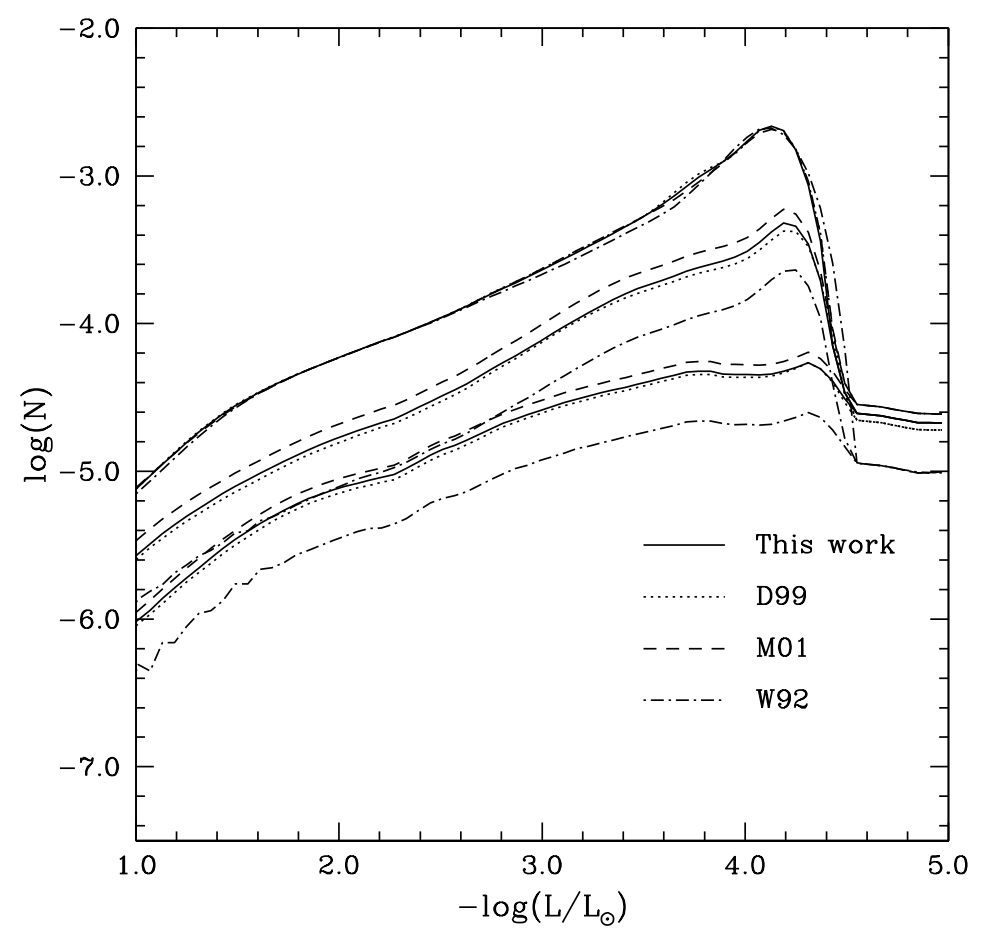

Figure 5.7: White dwarf luminosity functions using different initial-final mass relationships: Domínguez et al. (1999) - D99 - Marigo (2001) - M01 — Wood (1992) - W92 - and this work. From top to bottom we show the total luminosity function, and the luminosity functions of white dwarfs with masses larger than $0.7 M_{\odot}$ and $1.0 M_{\odot}$, respectively.

calculation we have considered an age of 11 Gyr for the Galactic disk. From top to bottom we show the total luminosity function and the luminosity functions of white dwarfs with masses larger than $0.7 M_{\odot}$ and $1.0 M_{\odot}$, respectively. As expected, the number of white dwarfs predicted decreases considerably as we restrict the sample to larger masses, especially in the case of Wood (1992).

Recently, Liebert et al. (2005a) performed high signal-to-noise spectroscopic observations of more than 300 white dwarfs belonging to the Palomar Green Survey. The analysis of this set of data has provided us with a sample of white dwarfs with well determined masses that allows for the first time the study of the white dwarf luminosity function of massive white dwarfs (Isern et al. 2007). Unfortunately, according to Liebert et al. (2005a) the completeness of the sample decreases severely near $10000 \mathrm{~K}$, where white dwarfs with small masses $\left(0.4 M_{\odot}\right)$ are brighter $\left(M_{\mathrm{V}} \sim 11\right)$ than massive white dwarfs with $M>0.8 M_{\odot}$ (which have visual magnitudes $M_{\mathrm{V}} \sim 13$ ). Thus, above $M_{\mathrm{V}}=11$ this survey only has detected white dwarfs with masses larger than $0.4 M_{\odot}$. Furthermore, below effective temperatures of about 


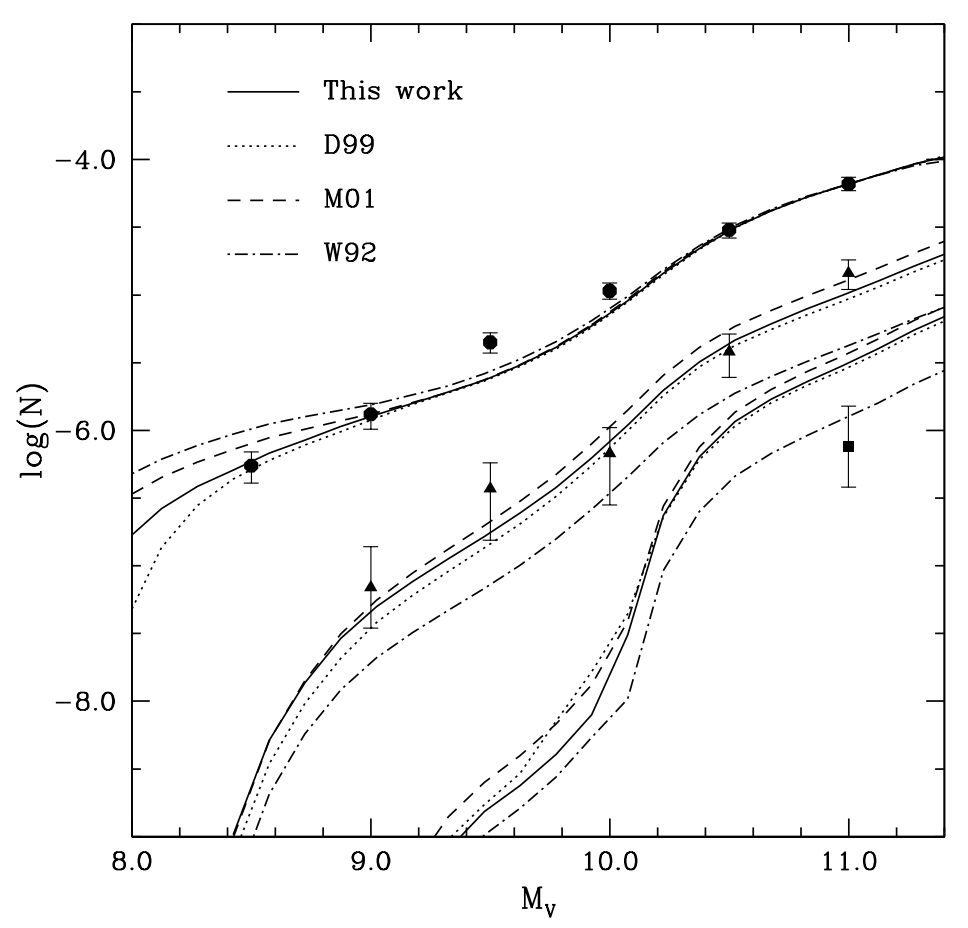

Figure 5.8: White dwarf luminosity functions versus visual magnitude using the same initialfinal mass relationships as in Fig. 5.7. From top to bottom we show the total luminosity function, and the luminosity functions of white dwarfs with masses larger than $0.7 M_{\odot}$ and $1.0 M_{\odot}$. Circles, triangles and squares correspond to the observational data of Liebert et al. (2005a).

$12000 \mathrm{~K}$, the determination of the mass is rather uncertain (Bergeron et al. 1992). Therefore, we will limit the analysis to white dwarfs brighter than $M_{\mathrm{V}} \sim 11$. We computed a set of white dwarf luminosity functions as done so far, but using bins of visual magnitude. The total luminosity function (considering all the range of masses) was normalized to the bin corresponding to $M_{\mathrm{V}}=11$, and then, this normalization factor was used for the luminosity functions of white dwarfs more massive than $0.7 M_{\odot}$ and $1.0 M_{\odot}$. The results obtained are shown in Fig. 5.8. In this case, we have used the stellar evolutionary inputs of Domínguez et al. (1999), Marigo (2001), Wood (1992) and also considered our semi-empirical initial-final mass relationship. Comparing the different theoretical luminosity functions, it can be noted that the predicted number of massive white dwarfs is considerable larger when using the inputs of Domínguez et al. (1999), Marigo (2001) and our semi-empirical initialfinal mass relationship in comparison with the results obtained when considering the expressions of Wood (1992). This is obviously due to the fact that the initial-final 


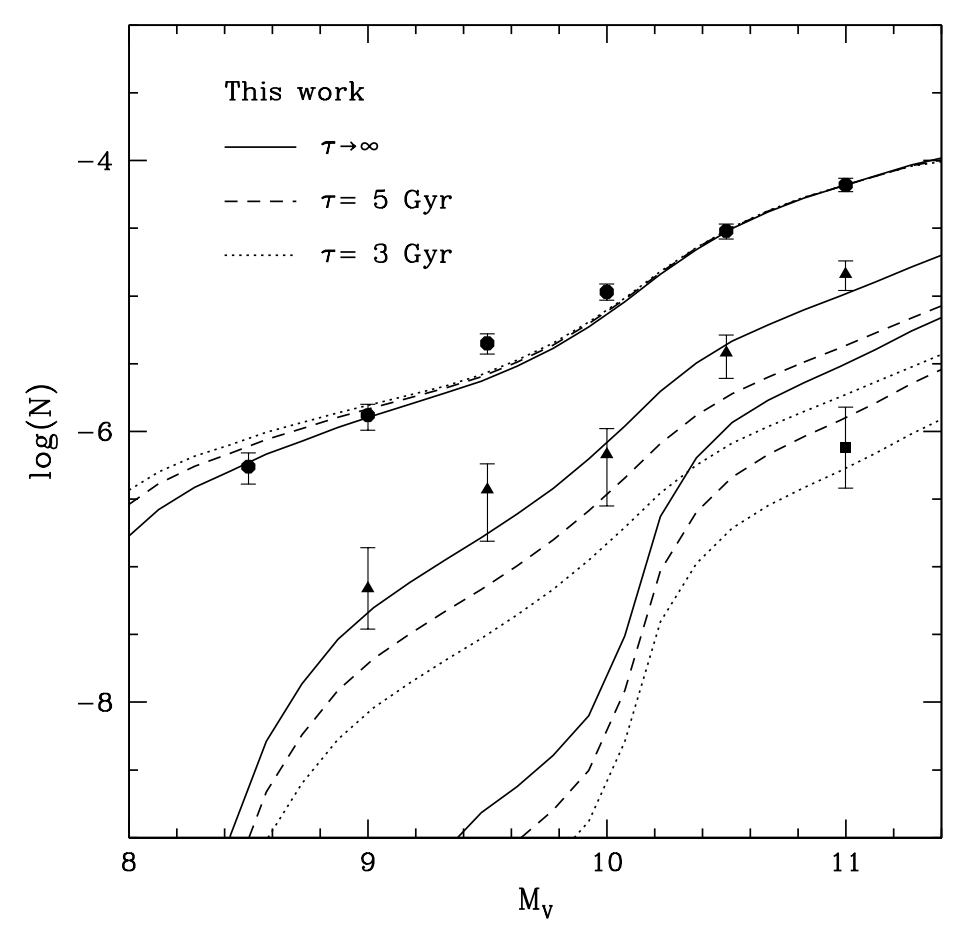

Figure 5.9: White dwarf luminosity functions versus visual magnitude using the initial-final mass relationship derived in this work and different star formation rates. Circles, triangles and squares correspond to the observational data of Liebert et al. (2005a).

mass relationship of Wood (1992) favors the production of low-mass white dwarfs. It can also be noted that the density of massive white dwarfs is slightly larger when considering the initial-final mass relationships of Marigo (2001) than that obtained when using the initial-final mass relationship derived here. The reverse is true when the initial-final mass relationship of Domínguez et al. (1999) is used. According to Fig. 5.1, to obtain a $1.0 M_{\odot}$ white dwarf it is necessary a progenitor with a mass of $5.0 M_{\odot}$ in the case of Marigo (2001) or our semi-empirical relation, $6.2 M_{\odot}$ in the case of Domínguez et al. (1999) and finally, 7.5 $M_{\odot}$ in the case of Wood (1992). Since the initial mass function assumed (Eq. 5.2) favors the production of low-mass white dwarfs, there will be less massive white dwarfs when considering the relation of Wood (1992) than in the rest of the cases, since they come from more massive progenitors. Without considering the results obtained when using the expressions of Wood (1992), it can be noted that it is not possible to evaluate which initialfinal mass relationship produces a theoretical luminosity function that fits better the observational data, since the error bars of the observational data are larger than the differences between the theoretical results. In any case, what can be clearly 


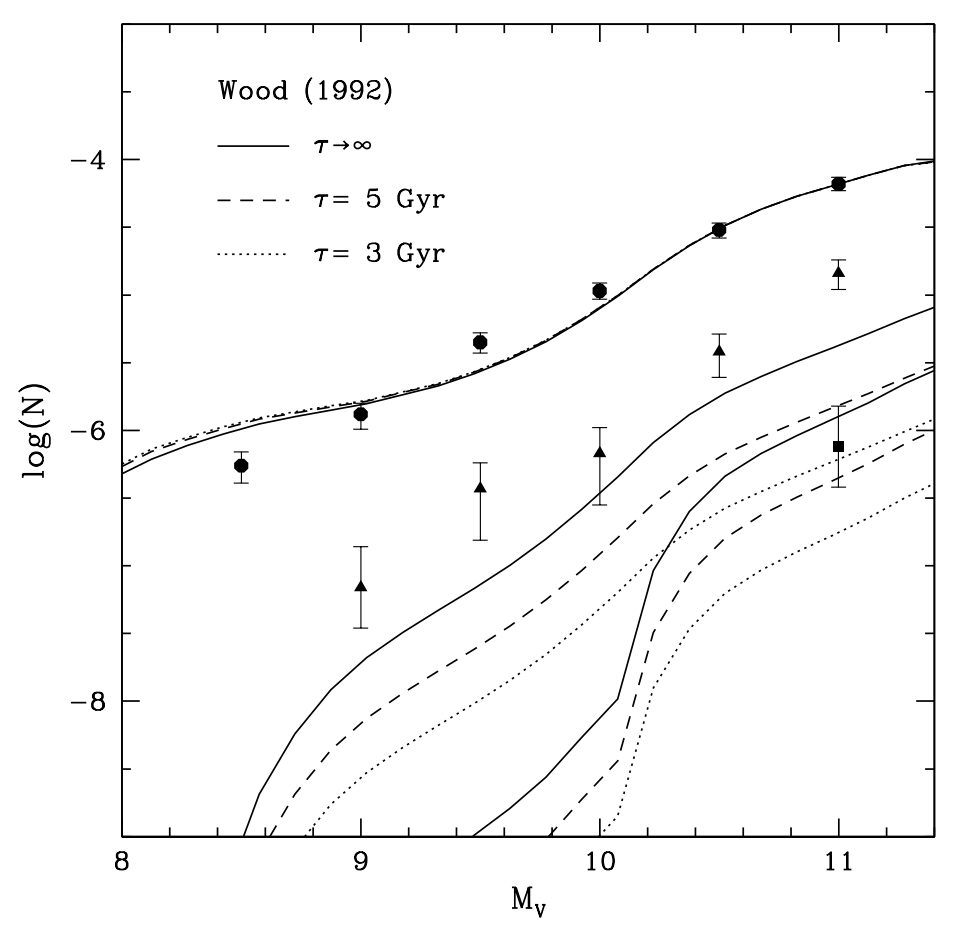

Figure 5.10: Same as Fig. 5.9 but using the initial-final mass relationship of Wood (1992).

seen is that all the theoretical relations predict more massive white dwarfs than the observations when a mass cut of $1.0 M_{\odot}$ is adopted, except in the case of Wood (1992). This can be due to the fact that we have considered a constant star formation rate. To evaluate the effect of the star formation rate on the white dwarf luminosity function we have repeated the calculations considering the initial-final mass relationship derived in last chapter and an exponentially decreasing star formation rate $\psi(T)=e^{(-T / \tau)}$, with $\tau=3$ and 5 Gyr. As it can be noted from Fig. 5.9 the production of massive stars drops considerably when a exponentially decreasing star formation rate is considered. The observational data corresponding to all the range of white dwarf masses and to masses larger than $0.7 M_{\odot}$ is better fitted when a constant star formation rate $(\tau \rightarrow \infty)$ is assumed in the theoretical calculations. On the contrary, for white dwarfs with masses larger than $1.0 M_{\odot}$ the agreement is better if we consider a variable star formation rate. However, for such massive stars there is only data for one magnitude bin. More observations corresponding to massive white dwarfs are needed to confirm this behavior of the luminosity function. For the sake of comparison we have carried out the same calculations but considering the expressions of Wood (1992), which are shown in Fig. 5.10. In this case, the 
observational data for stars with masses larger than $0.7 M_{\odot}$ is not fitted regardless of the star formation assumed. On the contrary, the fit is better when considering stars with masses above $1.0 M_{\odot}$. Since there are more observational data for the range of masses above $0.7 M_{\odot}$, we find more reliable the conclusions that can be obtained from a comparison of these data than from only one bin of magnitude, as in the case of massive white dwarfs. Thus, it seems clear that observations rule out the initial-final mass relationship of Wood (1992), and that the others initial-final mass relationships used in this work seem to be more reliable, although the present status of the observational data does not allow to draw a definitive conclusion about which of the three initial-final mass relationships is more adequate.

\subsection{Discussion}

In this chapter we have carried out a theoretical study to assess the impact of the initial-final mass relationship on the resulting white dwarf luminosity function. We have computed the luminosity functions of disk white dwarfs using different initialfinal mass relationships and the corresponding stellar tracks, when available. In all the cases, the results were similar, specially for the hot branch of the luminosity function. The fit of the cut-off in the luminosity function, which is located at $\log \left(L / L_{\odot}\right) \simeq-4.5$, yielded the same age for the Galactic disk in all the cases, $11 \mathrm{Gyr}$ approximately, except when the expressions of Wood (1992) were used, for which we obtained a shorter value (10.5 Gyr). This difference lies mainly on the behavior of the initial-final mass relationship of Wood (1992), which predicts less massive white dwarfs than the rest of the initial-final mass relationships.

Liebert et al. (2005a) performed an accurate determination of the masses of white dwarfs belonging to the Palomar Green Survey. This has allowed us to perform a preliminary evaluation of the influence of the initial-final mass relationship in the white dwarf luminosity function of massive white dwarfs. We have seen some differences between the theoretical luminosity functions computed, obtaining a larger density of massive white dwarfs when the initial-final mass relationship used favored the production of these stars, as expected. The first series of calculations were performed considering a constant star formation rate, and we always obtained a reasonable fit when white dwarfs more massive than $0.7 M_{\odot}$ were considered, except when we used the initial-final mass relationship of Wood (1992). For this last case the available observational data allowed us to discard this relationship. However, the error bars of the observational data are definetely larger than the differences between the theoretical luminosity functions which prevented us from favoring any of the remaining initial-final mass relationships. We have also shown that the production of massive white dwarfs is highly dependent on the assumed star formation rate, since in the case of an exponentially decreasing star formation rate the density of massive white dwarfs drops considerably. We have carried out these calculations 
using the semi-empirical initial-final mass relationship derived in chapter 4 and the relation of Wood (1992). The results obtained are remarkably different, and in the case of Wood (1992), the observational data corresponding to white dwarfs with masses above $0.7 M_{\odot}$ is not fitted by the theoretical luminosity functions, regardless of the star formation rate adopted.

Given the presently available observational data, any attempt to discern which initial-final mass relationship better fits the data is not feasible. However, our results favor an initial-final mass relationship that produces more massive white dwarfs than the relation of Wood (1992). Thus, we consider that all the initial-final mass relationships used in this work, except that of Wood (1992), are valid to compute reliable theoretical luminosity functions. This is an important finding, since the initial-final mass relationship of Wood (1992) is the most commonly used relationship for computing the theoretical white dwarf luminosity function. 


\section{Chapter 6}

\section{An optical survey: the Alhambra Survey}

Since the earliest times of astronomy, the history, structure and origin of our Galaxy has been a subject of debate. How the Galaxy was formed and the way in which it has evolved up to the actual moment is still something that it is not yet well settled. However, nowadays the technological facilities necessary to disentangle such a relevant matter are becoming available. In particular, there are several new space missions - of which GAIA, SIM, and Kepler are leading examples - and ground deep surveys - like LSST, SDSS, PAN-Starrs and the Alhambra Survey — that will benefit our current understanding of our Galaxy. These projects will provide very deep surveys of the entire sky, which will enable us not only to test the theories of galaxy formation but also will help in building a knowledge of the nature and origin of the universe itself. This is because these surveys will provide unprecedented information on millions of stars in our Galaxy and throughout the Local Group. These data will have the precision necessary to quantify the early formation, and subsequent dynamical, chemical and star formation evolution of the Milky Way.

In this regard it is important to note that thanks to the depth of these surveys, thousands of new white dwarfs will be detected, some of them belonging to wide binaries as the ones previously studied in chapter 2, which will help to improve the initial-final mass relationship. These surveys will offer also the possibility of observing the white dwarf population beyond the cut-off in the luminosity function, reported by Liebert et al. (1988) and Oswalt et al. (1996), and also to discriminate - by taking into account their kinematical properties - those white dwarfs which possibly belong to the halo of our Galaxy. Moreover, it is thought that the halo was formed sometime before the disk as a burst of short duration (Eggen et al. 1962). If this assumption is true, it would be possible to obtain information about the time elapsed between the creation of both structures through the study of these old stars (Isern et al. 1998). 


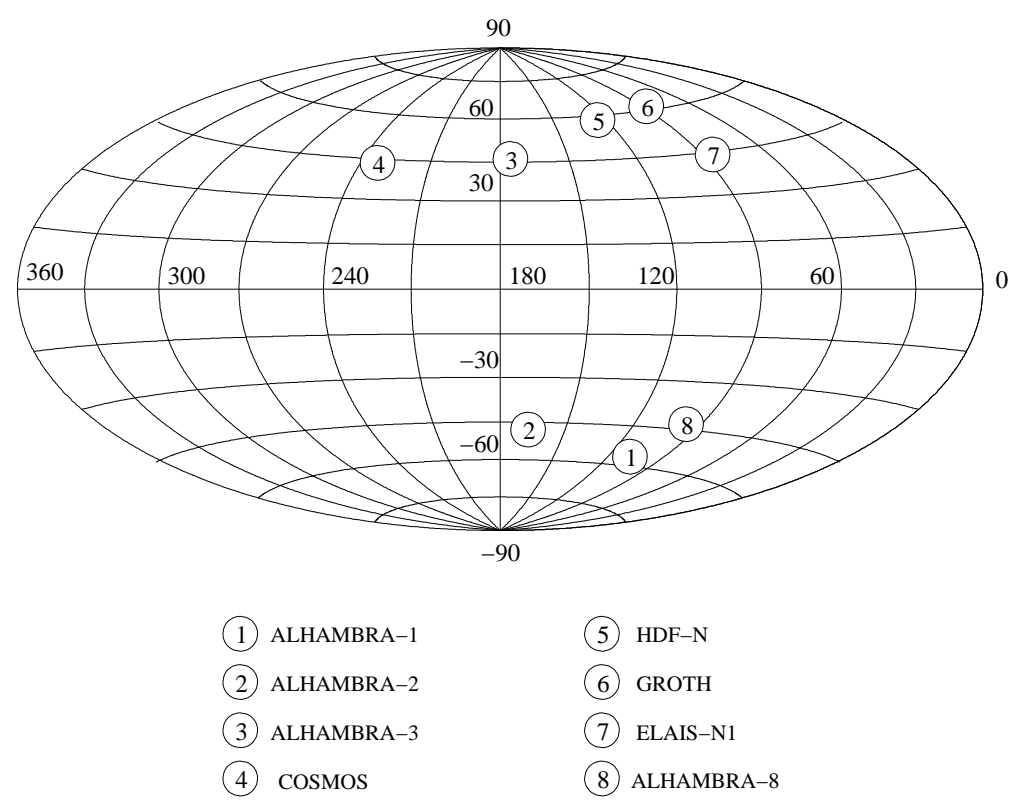

Figure 6.1: Fields chosen by the Alhambra team, represented in Galactic coordinates.

In this chapter we present the Alhambra Survey, in which we are actively involved, and we show some predictions regarding its potential contribution to the knowledge of the white dwarf population.

\subsection{Description of the project}

The ALHAMBRA (Advanced Large, Homogeneous Area Medium Band Redshift Astronomical) survey will observe eight 1-squared degree fields, distributed in both galactic hemispheres to allow continuous observations throughout the year. Fig. 6.1 shows the location of the proposed fields. They have been selected taking into account different aspects. Some of them have been chosen intentionally to overlap with other publicly available surveys for the sole purpose of verifying calibration methods and comparing results. The other fields have been selected close to the celestial equator to allow future follow-up research in both hemispheres. Table 6.1 shows the equatorial and galactic coordinates of each of the Alhambra fields.

The Alhambra survey is a general-purpose survey whose primary aim is to provide a large area of 8 square degrees, magnitude-limited photometric catalogue in $20+3$ bands, which will include reliable and precise photometric redshifts for about 1 million galaxies and Active Galactic Nuclei. The images will be taken by the camera LAICA at the Calar Alto $3.5 \mathrm{~m}$ telescope using a constant-width, marginally 
Table 6.1: Equatorial and Galactic coordinates for the Alhambra fields.

\begin{tabular}{|c|c|c|c|c|}
\hline Field & $\alpha$ & $\delta$ & $l$ & $b$ \\
\hline ALHAMBRA-1 & 002946.0 & +052528 & 113 & -57 \\
\hline ALHAMBRA-2 & 022832.0 & +004700 & 166 & -53 \\
\hline ALHAMBRA-3 & 091620.0 & +460220 & 174 & 44 \\
\hline COSMOS & 100028.6 & +02 1221 & 236 & 42 \\
\hline HDF-N & 123455.0 & +615010 & 125 & 55 \\
\hline GROTH & 142032.0 & +525805 & 95 & 60 \\
\hline ELAIS-N1 & 161000.0 & +540000 & 83 & 45 \\
\hline ALHAMBRA-8 & 234550.0 & +153450 & 99 & -44 \\
\hline
\end{tabular}

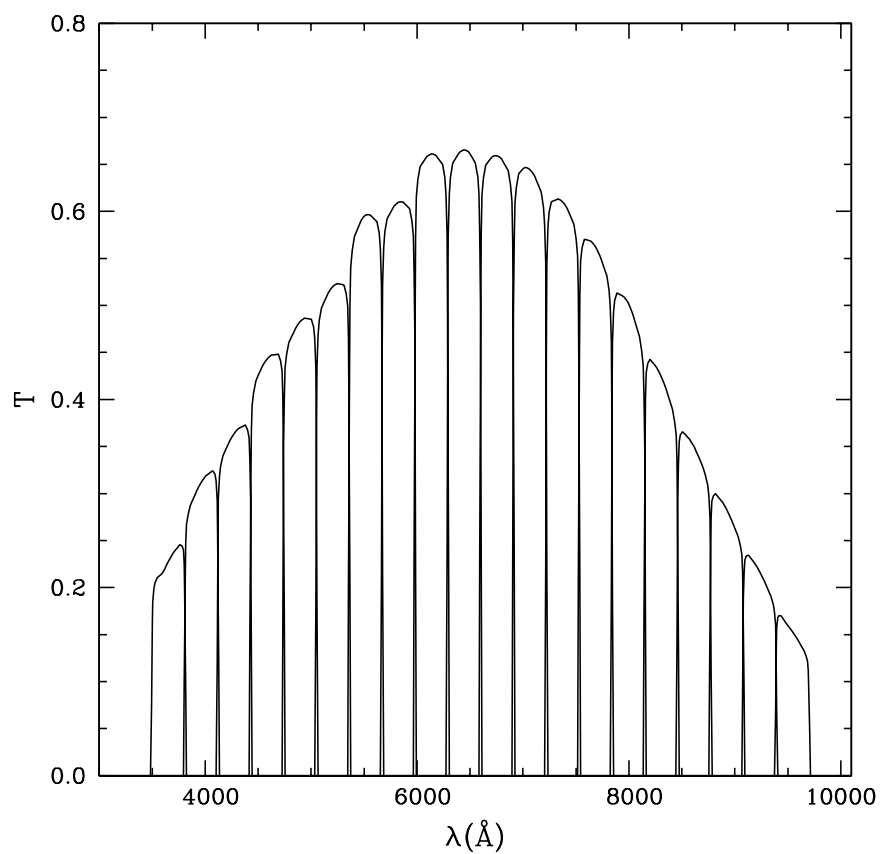

Figure 6.2: Transmission function for each of the ALHAMBRA filters as a function of wavelength. 


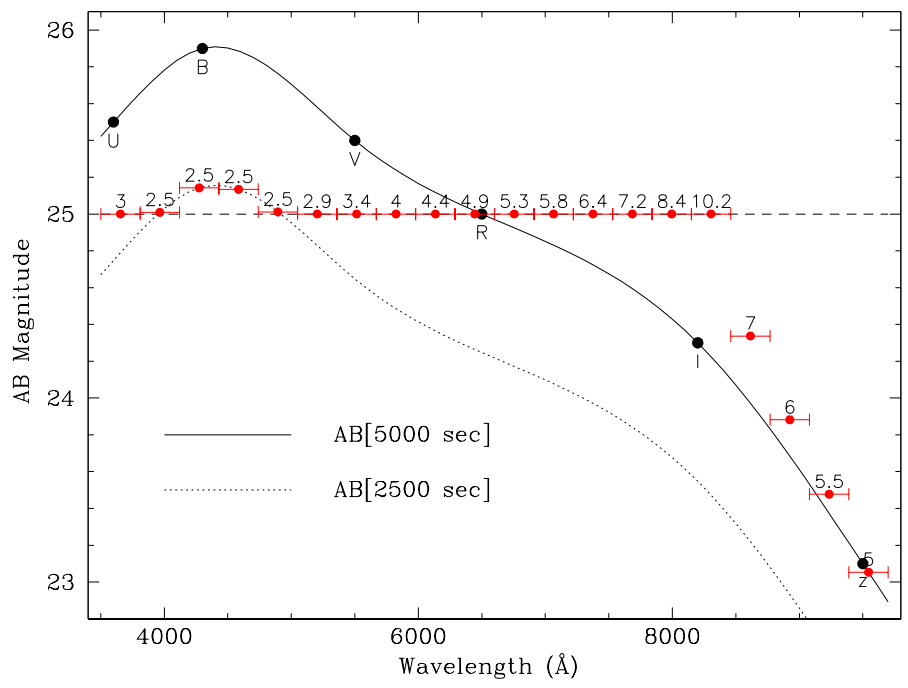

Figure 6.3: AB limit magnitude versus wavelength for different exposure times.

overlapping, medium band, top-hat 20-filter system in order to provide a complete, contiguous spectral coverage in the optical wavelength range (3500-9700 $\AA$ ), and also $J H K$ observations with the Omega Prime camera at the same telescope. Although this survey is especially well suited for the analysis of cosmic evolution (large scale structure, galaxy luminosity function, galactic population, supernovae, Quasi Stellar Objects,...) it will also be helpful to detect new white dwarfs. The purpose of this chapter is to estimate the magnitudes that white dwarf candidates will have after observing them with the 20 non-overlapping filter system previously mentioned. For this purpose, we will use theoretical white dwarf spectra and the transmission of each filter. Fig. 6.2 illustrates the response of the filters as a function of their respective wavelength. This transmission takes also into account the typical atmospheric extinction at Calar Alto. As can be noticed, the filters are well behaved and, although there is some overlapping the effect on the results will be almost negligible.

In Fig. 6.3, the limiting $A B$ magnitude as a function of wavelength is shown for each one of the filters in the optical domain. The goal of this survey is to reach in the optical range a constant flux, $A B=25$ at $\mathrm{S} / \mathrm{N}=5$, in all the filters from 3500 to $8300 \AA$ and from $A B=24.7$ to 23.4 for the remaining filters. The exposure time necessary to reach the quoted limit has been computed for each filter and it is indicated in ksecs in each of the bins. The solid and the dotted line show, respectively, the $A B$ magnitudes that would be reached with an exposition of 2500 and 5000 seconds.

A comparison of the main characteristics of the Alhambra survey and those of some other publicly available surveys is shown in Table 6.2. Particularly, we 
Table 6.2: Specific characteristics of different surveys.

\begin{tabular}{lcclc}
\hline \hline Survey & $m_{\lim }$ & Area $\left(\mathrm{o}^{2}\right)$ & Spectral Range & Resolution \\
\hline COSMOS & 27.0 & 2 & $g I$ & 2.5 \\
HDF-N & 28.0 & 0.001 & UVI & 4.0 \\
SDSS & 21.3 & 8000 & Ugriz & 6.0 \\
NOAO & 26.0 & 18 & BRIJHK & 4.0 \\
COMBO-17 & 24.0 & 1 & $3650-9140 \AA$ & 25.0 \\
ALHAMBRA & 25.0 & 8 & $3500-22000 \AA$ & 25.0 \\
\hline \hline
\end{tabular}

compare the characteristics of the Cosmological Evolution Survey (first entry), the Hubble Deep Field North (second row), the Sloan Digital Sky Survey (third row), the National Optical Astronomical Observatory Deep Survey (fourth entry) and the COMBO-17 (fifth entry) survey. The last entry in Table 6.1 corresponds precisely to the Alhambra survey. For each of the above mentioned surveys we display the limiting magnitude, the area surveyed (in square degrees), the spectral range and the spectral resolution. As can be seen in Table 6.2 the Alhambra survey will not be the deepest of these surveys whatsoever. Nevertheless, it is deeper than the COMBO-17 survey and the Sloan Digital Sky Survey (SDSS). The most salient feature of the Alhambra survey is that it will provide observations over a large area, as well as a high resolution. These two characteristics are unrivaled by the rest of the surveys, thus making the Alhambra survey an important tool for probing the properties of the white dwarf populations.

\subsection{Photometric identification of white dwarfs}

In this section we have used a set of theoretical spectra for DA white dwarfs to compute $A B$ magnitudes. These models have been kindly provided to us by D. Koester and range from 7000 to $60000 \mathrm{~K}$ in effective temperature and from $10^{7}$ to $10^{9} \mathrm{~cm} / \mathrm{s}^{2}$ in surface gravity. In order to compute the magnitude in the $A B$ system of these spectra for each of the filters we need the flux observed from the Earth and, since these spectra give the mean intensity of the stellar disk, some calculations need to be done. Assuming a distance of $10 \mathrm{pc}$ it is possible to obtain the absolute observed flux taking into account the following expression:

$$
F_{\lambda}=\frac{\pi R^{2}}{D^{2}} I_{\lambda}
$$

where $D$ is the distance to the source, $I_{\lambda}$ is the mean intensity of the stellar disk, and $R$ is the radius of the star, which can be obtained from

$$
g=\frac{G M}{R^{2}}
$$



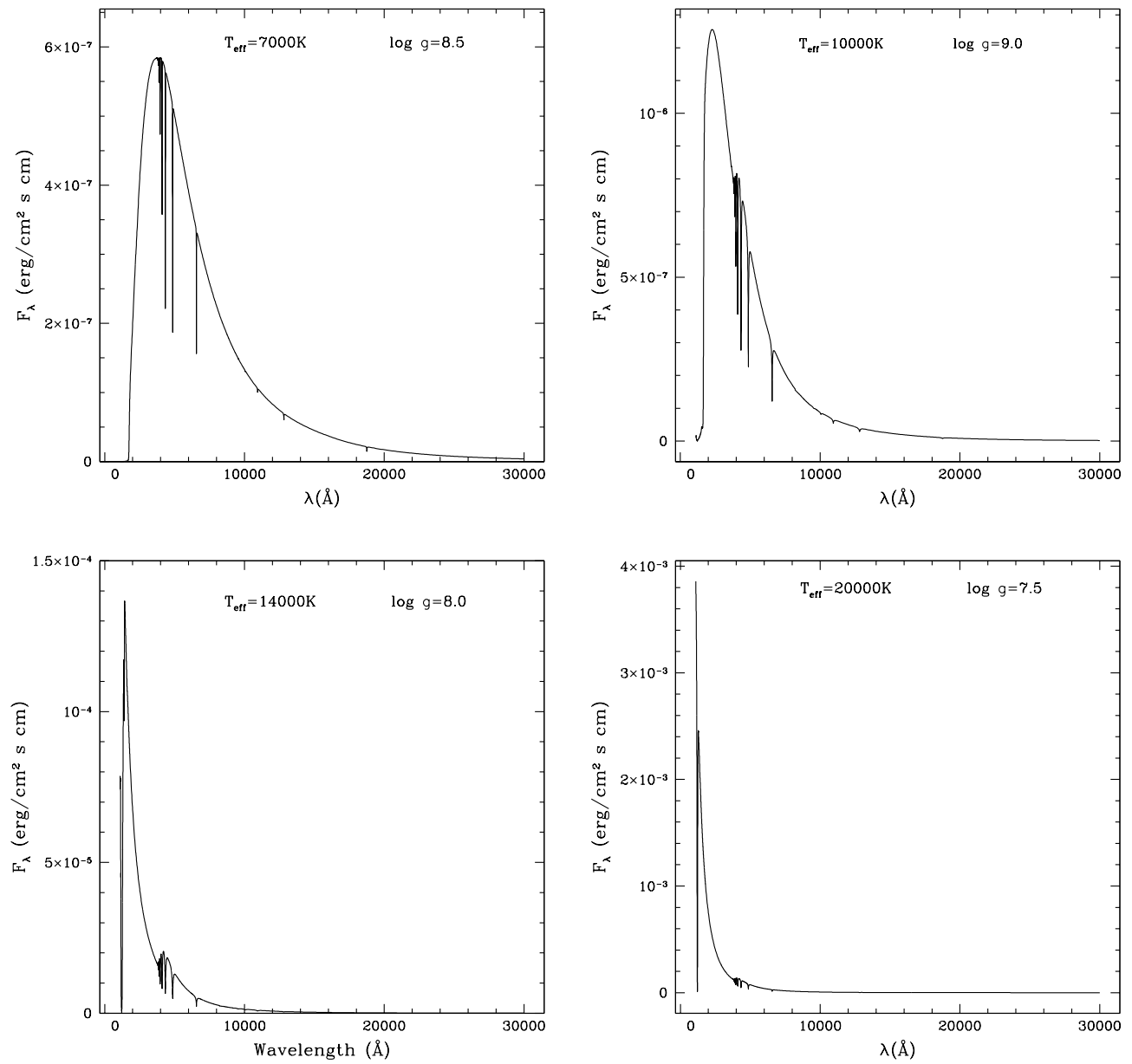

Figure 6.4: Theoretical flux versus wavelength for different DA white dwarfs.

being $g$ the surface gravity, $G$ the gravitational constant, and $M$ the mass of the star. In Fig. 6.4 we show some theoretical spectra of DA white dwarfs with different temperatures and surface gravities. The effective temperatures and surface gravities can be found in the top legend of each one of the panels, and are representative of typical white dwarfs. It can be noted how the emission peak moves to smaller wavelengths when the effective temperature is larger. The uppermost left panel shows the spectrum of a DA white dwarf with $T_{\text {eff }}=7000$ and $\log g=8.5$, the uppermost right panel shows the spectrum for the case in which $T_{\text {eff }}=10000 \mathrm{~K}$ and $\log g=9.0$ - a rather massive and cool white dwarf. On the other hand, the lower left panel shows the spectrum of an otherwise typical hot white dwarf ( $T_{\text {eff }}=14000$ and $\log g=8.0$ ), and the lower right panel of Fig. 6.4 shows the spectrum of a low- 

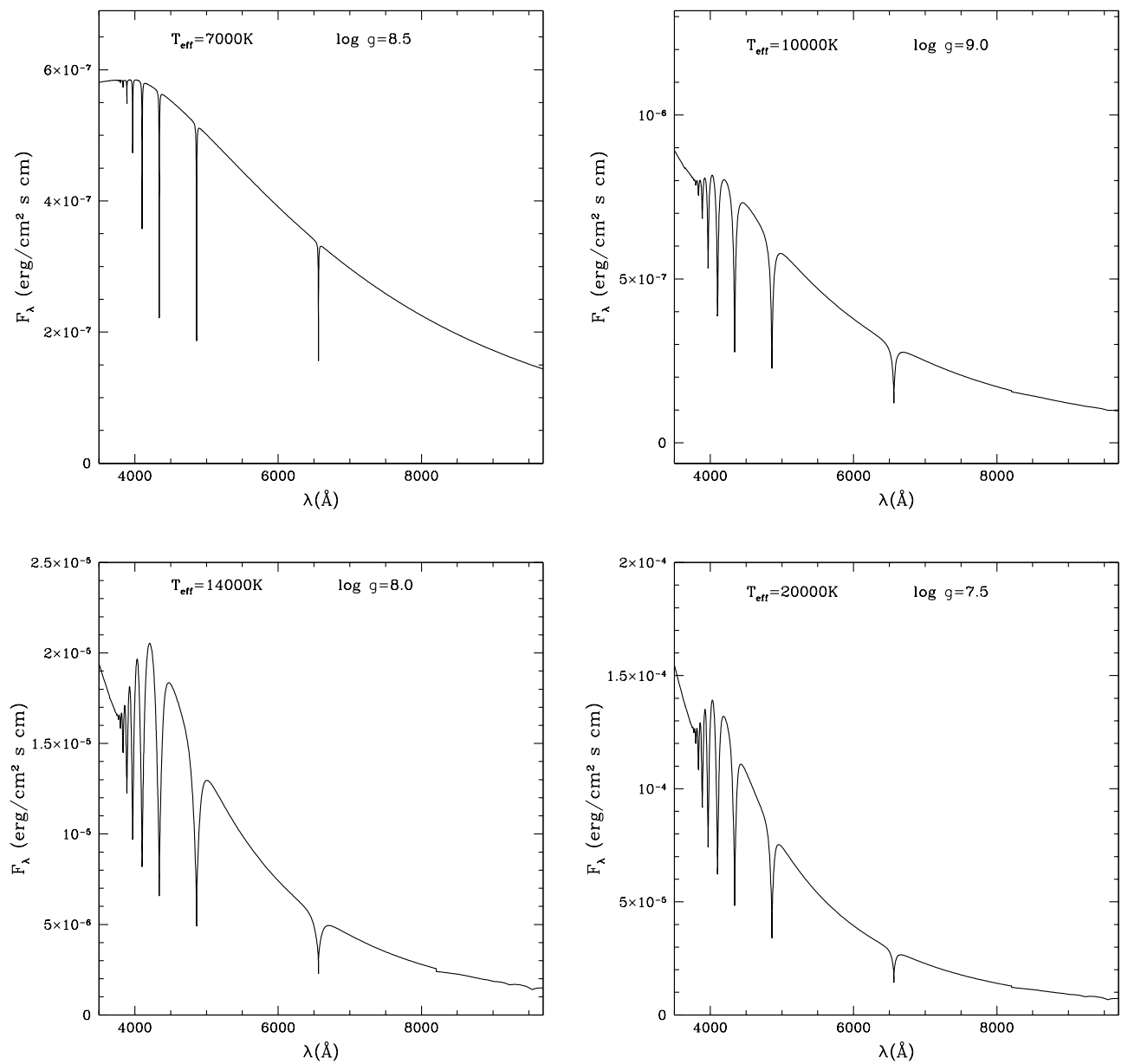

Figure 6.5: Theoretical flux versus wavelength for different DA white dwarfs (zoom).

mass very hot white dwarf with $T_{\text {eff }}=20000$ and $\log g=7.5$. In order to appreciate the different absorption lines and other structures that are of interest, in Fig. 6.5 we have represented an expanded view of the same spectra, showing only the region in which the most relevant absorption features appear, that is, from 3500 to $9700 \AA$. As it can be seen the most obvious hydrogen absorption lines, such as $\mathrm{H}_{\alpha}$ (at $6563 \AA$ ), $\mathrm{H}_{\beta}$ (at $\left.4861 \AA\right), \mathrm{H}_{\gamma}\left(\right.$ at $4340 \AA$ ), and $\mathrm{H}_{\delta}$ (at $3970 \AA$ ) are now clearly visible in these spectra.

Finally, in Fig. 6.6 we have represented a theoretical spectra of a white dwarf with an effective temperature of $10000 \mathrm{~K}$ and a surface gravity of $10^{8} \mathrm{~cm} / \mathrm{s}^{2}$ - which is fairly representative of a typical white dwarf - together with the grid of filters. The absorption lines $\mathrm{H}_{\gamma}$ and $\mathrm{H}_{\beta}$ are located in the third and fifth filter, respectively. 


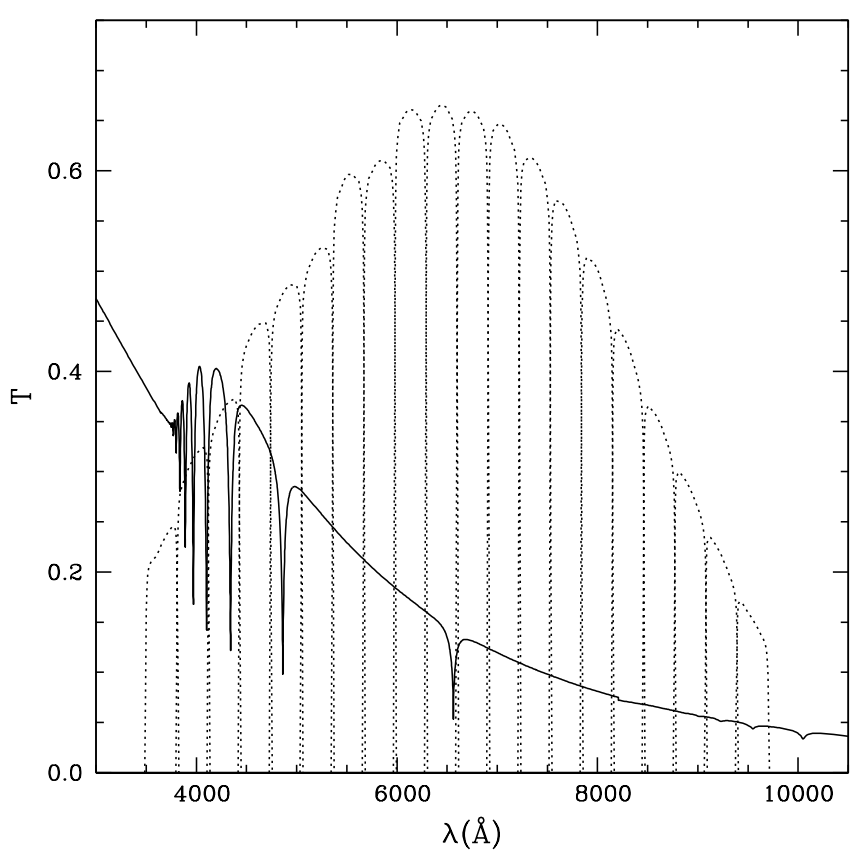

Figure 6.6: Theoretical flux and grid of filters versus wavelength.

The $\mathrm{H}_{\alpha}$ absorption line is located in the tenth filter, but it is not well centered, and consequently, the magnitude obtained in this case will not account for all the flux which corresponds to the wings of this absorption line. More specifically, only will account for one of them, the blue one. In the third filter the $\mathrm{H}_{\delta}$ line can be found and, probably in the second filter, other hydrogen transitions could be also detected. In the rest of the filters no representative line can be found. It must be noticed that we are working with DA white dwarf spectra, and as previously pointed out, this type of white dwarfs only show the absorption lines of hydrogen.

The brightness of a star, $b$, is defined as:

$$
b=\int_{\lambda_{1}}^{\lambda_{2}} F(\lambda) A_{\lambda}(z) R(\lambda) d \lambda
$$

where $F(\lambda)$ is the observed flux of the object in each wavelength, $A_{\lambda}(z)$ is the fraction of flux transmitted through the atmosphere, which depends on wavelength and on the zenital angle, $z$, and $R(\lambda)$ is the response of the system formed by the detector and the filter. As already seen before, this function is only defined in a wavelength interval, so we will need to integrate numerically $R(\lambda)$ between the wavelength limits of each filter, $\lambda_{1}$ and $\lambda_{2}$. Notice that in our calculations we are considering directly 

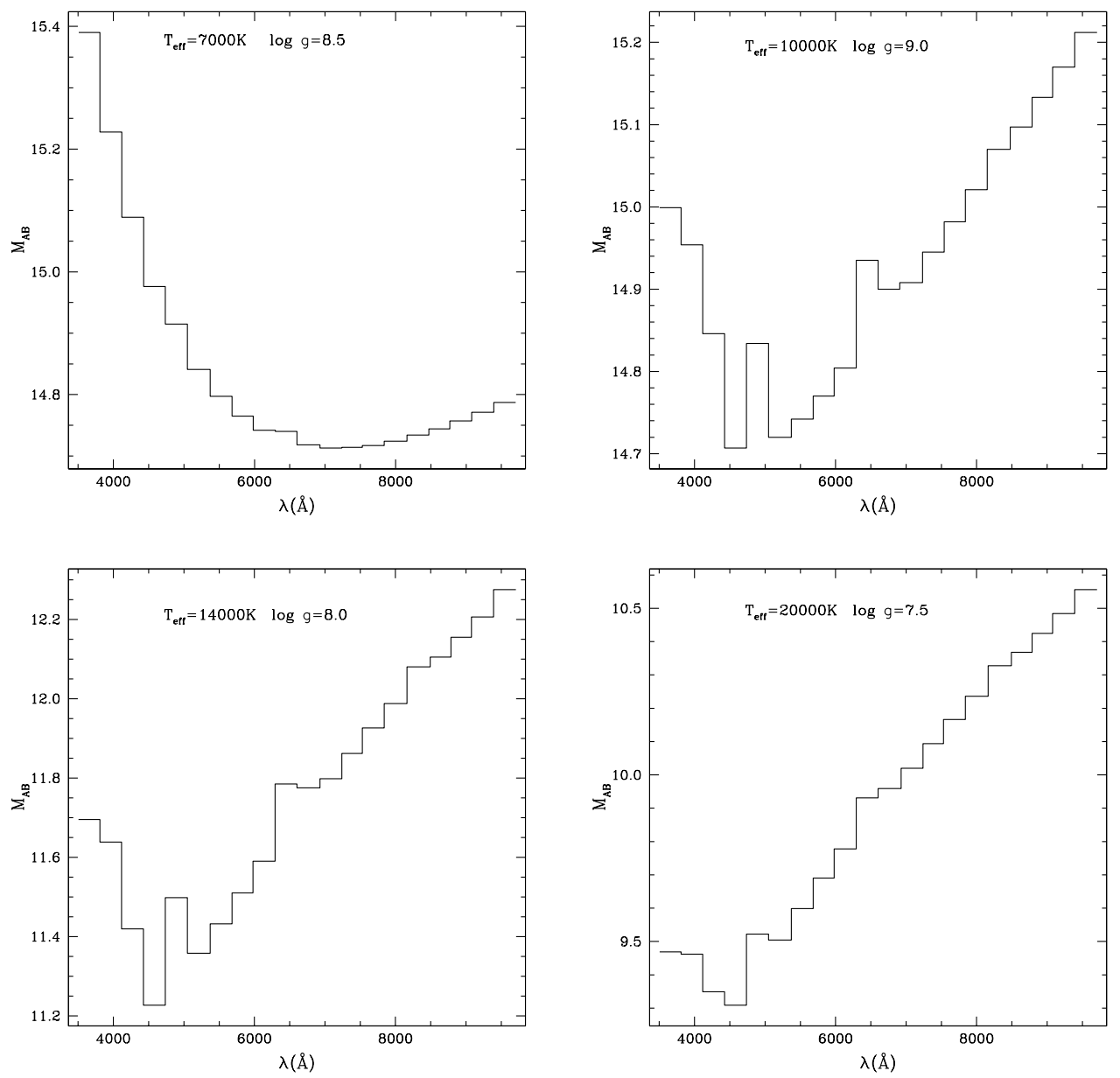

Figure 6.7: $A B$ magnitudes versus wavelength for different white dwarfs. See text for details.

the transmission of the filters (Fig. 6.2), which is defined as the product of the atmosphere extinction and the response of the system:

$$
T_{\lambda}(z)=A_{\lambda}(z) R(\lambda)
$$

Considering a distance of $10 \mathrm{pc}$, and taking into account Eq. (6.3) the absolute magnitude of the star in a given wavelength range can be easily obtained:

$$
M_{A B}=-2.5 \log (b)-48.6
$$

where $M_{A B}$ is the absolute magnitude in the $A B$ photometric system. We remind the reader that the magnitude in the $A B$ system is defined in such a way that an 

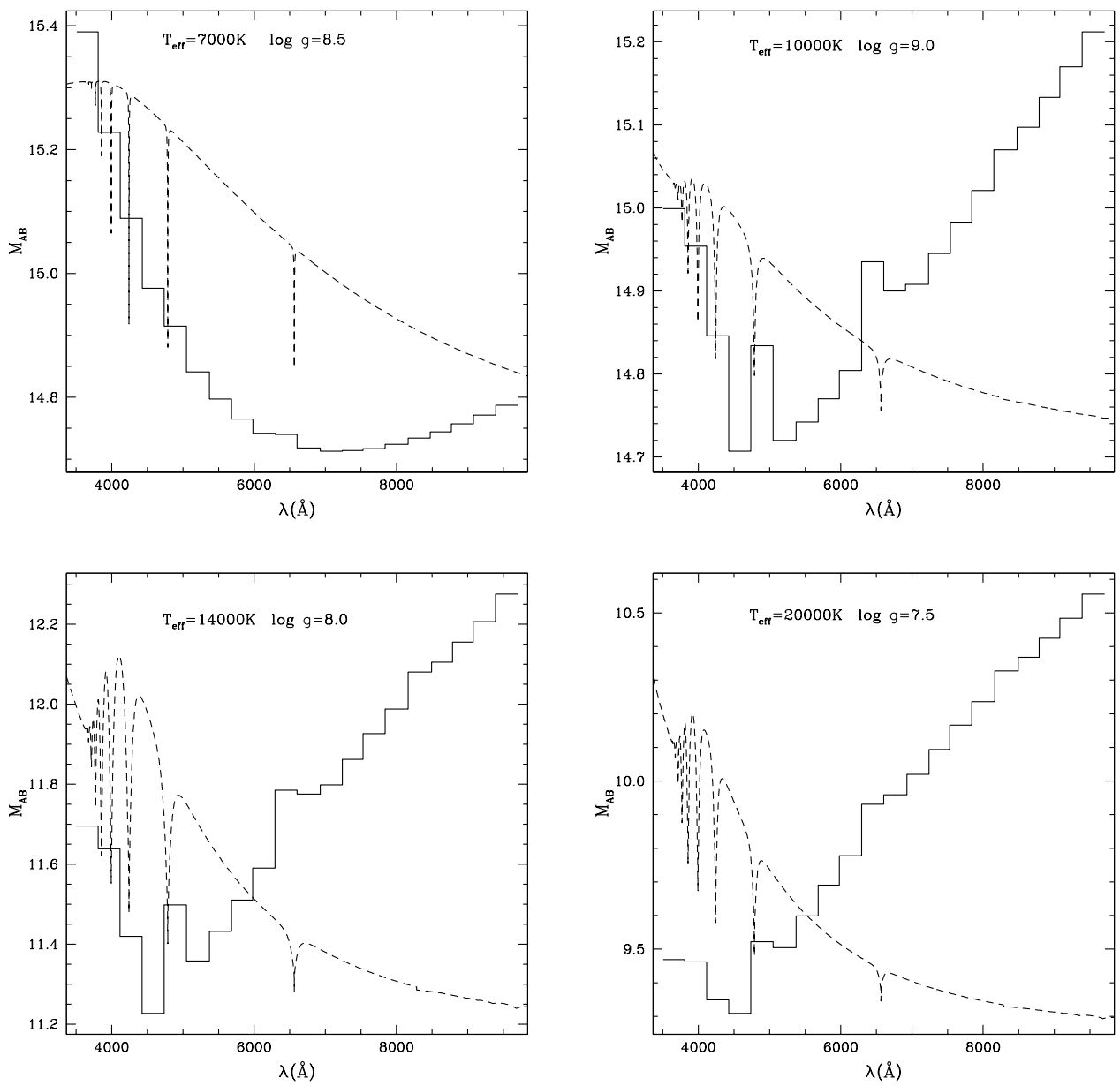

Figure 6.8: Flux and magnitude versus wavelength for different white dwarf spectra.

object with constant flux (flat energy distribution) has the same magnitude in all bands, and all colours are zero (Oke \& Gunn 1983).

Fig. 6.7 shows the hystogram of $A B$ magnitudes obtained after the process described before. As can be seen, the typical values are from 9 to 15 . Considering the limiting value of the Alhambra Survey $(\mathrm{AB}=25)$ all white dwarfs with a distance modulus below 10, i. e., located at less than $1 \mathrm{kpc}$, will be detected in this survey.

In Fig. 6.8, the flux of the white dwarf and its magnitude for each wavelength are represented. It can be seen that when the flux decreases (due to the absorption lines) the magnitude increases, as expected. 


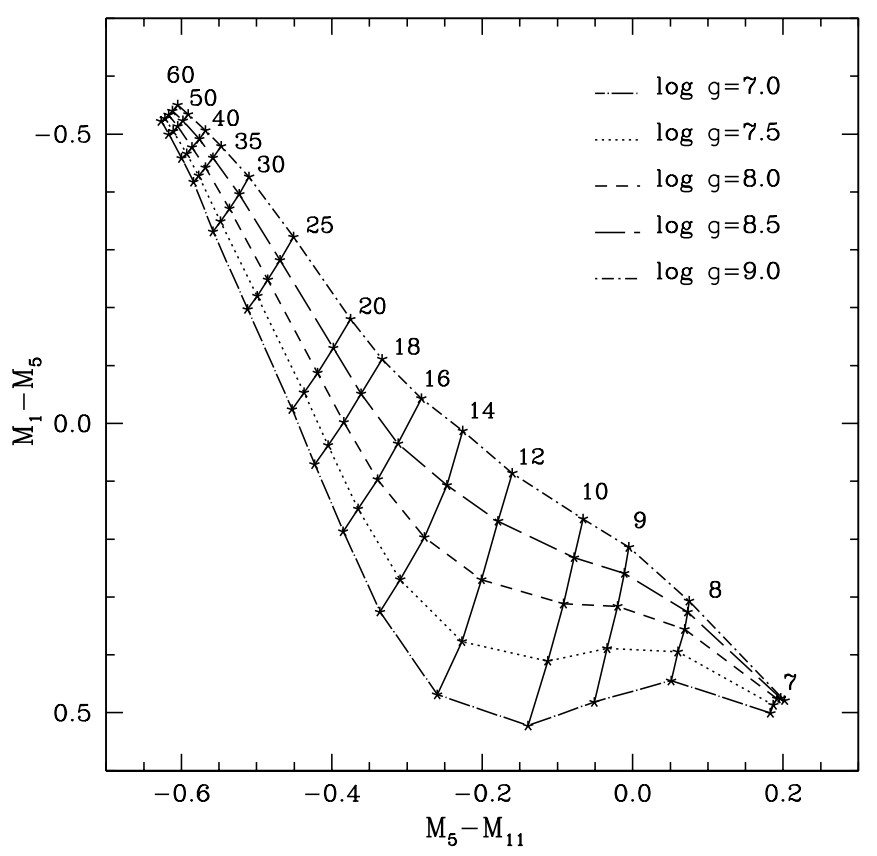

Figure 6.9: Color-color diagram for the filters 1, 5 and 11.

\subsubsection{Color-color diagrams}

The Alhambra survey will detect different types of stars, although it is especially well suited for the detection of galactic structures and galaxies. The point in using the Alhambra survey as a provider of white dwarfs is the fact that galaxy colors are rather similar to those of white dwarfs. However, precisely because the colors of white dwarfs and galaxies are quite similar it is also true that it is necessary to be able to reliably distinguish both types of objects. This can be done by using color-color diagrams, a technique widely used today in other surveys of similar characteristics.

Since we alredy have the magnitudes for different white dwarf spectra (that is, for different temperatures and surface gravities) and for the different filters of the photometric system of the Alhambra survey, we are able to build a diagram in which we can represent the different colors. We tried different possibilities, since 20 magnitudes are available, but we have chosen those color-color diagrams for which we have obtained a wide grid. In this way, once the colors of white dwarfs are observationally determined we will be able to assign them quite easily an effective temperature and a surface gravity. On the contrary, if the diagram is narrow, or the lines of constant surface gravity cross each other, this association will not be possible. This is a fundamental step, because once the atmospheric parameters $T_{\text {eff }}$ and $\log g$ 


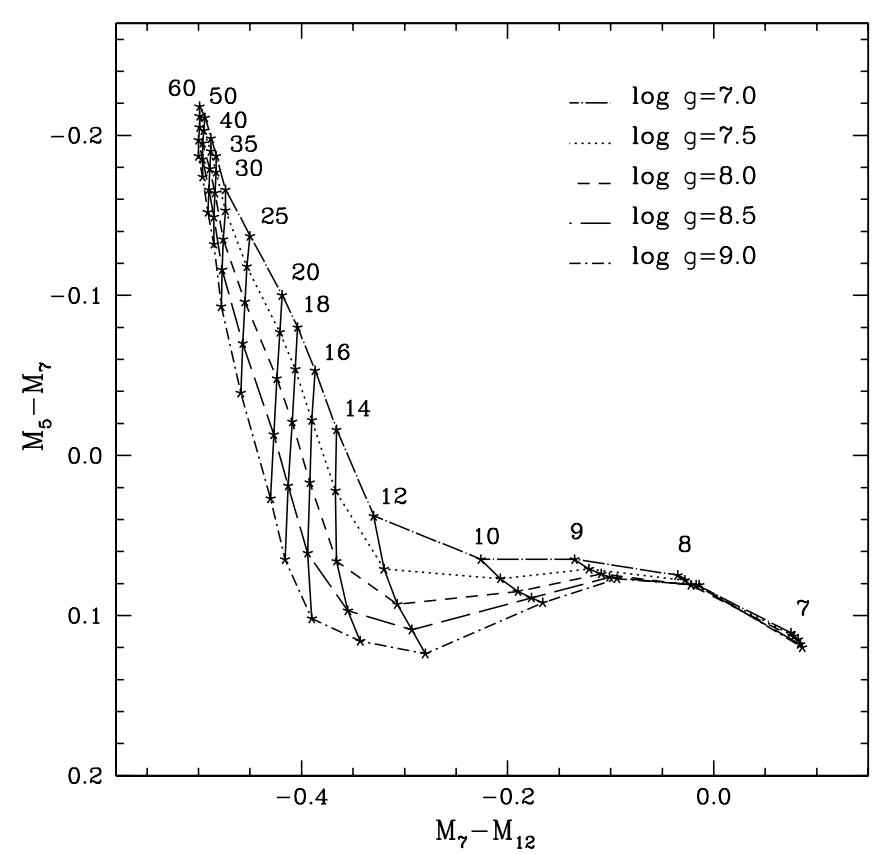

Figure 6.10: Color-color diagram for the filters 5, 7 and 12.

are obtained, other very important characteristics like the mass, radius, cooling time and total age of the white dwarf can be readily obtained from the proper cooling tracks.

In Fig. 6.9 we have represented the color-color diagram obtained using the filters 1, 5 and 11 of the Alhambra survey, which we denominate $M_{1}, M_{5}$, and so on... The coding for the lines of constant surface gravity can be found in the upper right legend of the figure. On its hand, the lines of constant effective temperature, which are almost perpendicular to the lines of constant $\log g$ are labelled in thousands of K. By studying Fig. 6.6 it is easy to realize that this set of filters accounts for two regions of the spectrum which are dominated by the continuum (filters 1 and 11) and the region which corresponds to the $\mathrm{H}_{\beta}$ absorption line (filter 5). This line is precisely very sensitive to both the effective temperature and the surface gravity and, thus, this is the reason why this color-color diagram is so well behaved. As a consequence, this diagram will be very useful to identify the characteristics of those observed white dwarfs with temperatures below 30000 K. In Fig. 6.10 the color-color diagram obtained using the filters 5,7 and 12 can be found. In this diagram we are taking into account the $\mathrm{H}_{\beta}$ absorption lines (filter 5) and two continuum regions (filters 7 and 12). Note that in this case the lines of effective temperatures are 

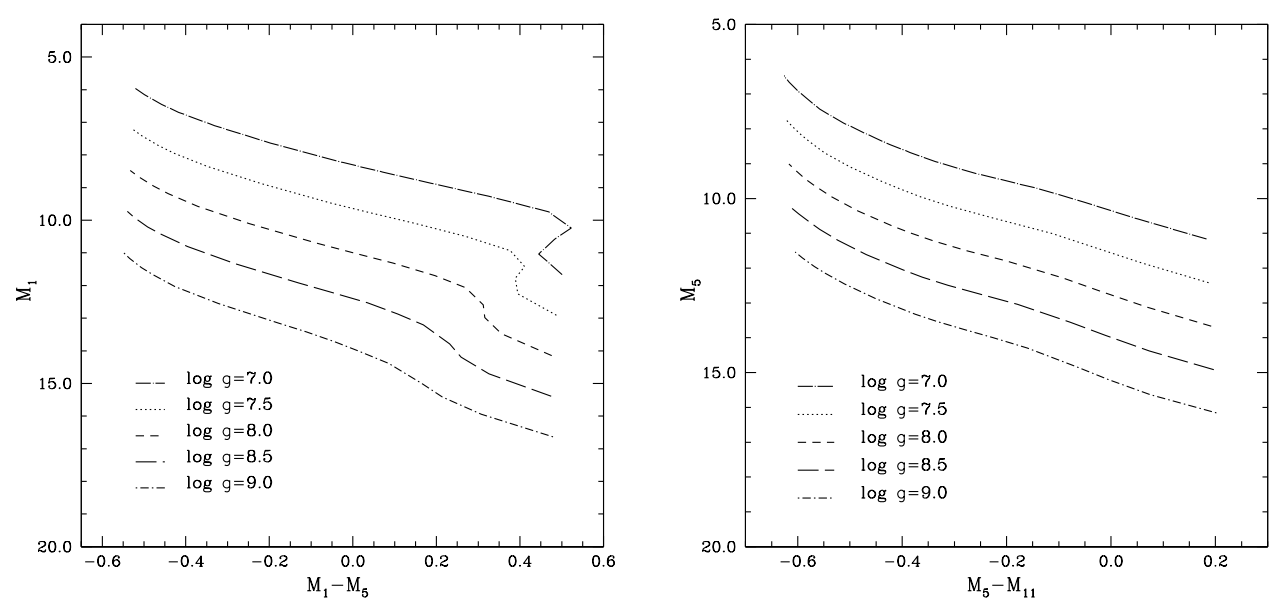

Figure 6.11: Color-magnitude diagrams for filters 1, 5 and 11.

almost overlapped in the zone corresponding to high effective temperatures, and it becomes difficult to obtain significative information of an observed white dwarf there. However, the grid is very well defined in the region corresponding to white dwarfs with temperatures between 10000 and $30000 \mathrm{~K}$. Thus, this color-color diagram can be used as a secondary indicator.

\subsubsection{Color-magnitude diagrams}

As explained in chapter 1, white dwarfs have a characteristic distribution in the Hertzsprung-Russell diagram. Figure 6.11 shows two color-magnitude diagrams. The adopted magnitudes are again $M_{1}$ (left panel) and $M_{5}$ (right panel) versus the corresponding colors: $M_{1}-M_{5}$ and $M_{5}-M_{11}$, respectively, as it was done in the previous section. As can be noticed, the left panel of Fig. 6.11 shows almost a linear distribution for the hottest white dwarfs. For the cooler ones this behavior disappears and it becomes difficult to distinguish between different surface gravities (which are clearly indicated on top of each curve). This behavior is due to the effect of the sharp change in the width and depth of the absorption line in the filter number 5 . The right panel of Fig. 6.11 shows an homogeneous distribution along the diagonal line, due to the abrupt decrease of the flux between the regions corresponding to these filters ( 5 and 11). In both figures it can be seen that magnitudes are fainter at higher temperatures and surface gravities.

In Fig. 6.12 we also plot other color-magnitude diagrams, analogous to those of Fig. 6.11, but in this case using the filters number 5, 7 and 12. Again, each curve is labelled with its corresponding surface gravity. In the left panel of Fig. 6.12 it can be seen the same behavior previously found: an almost linear distribution, except for the 

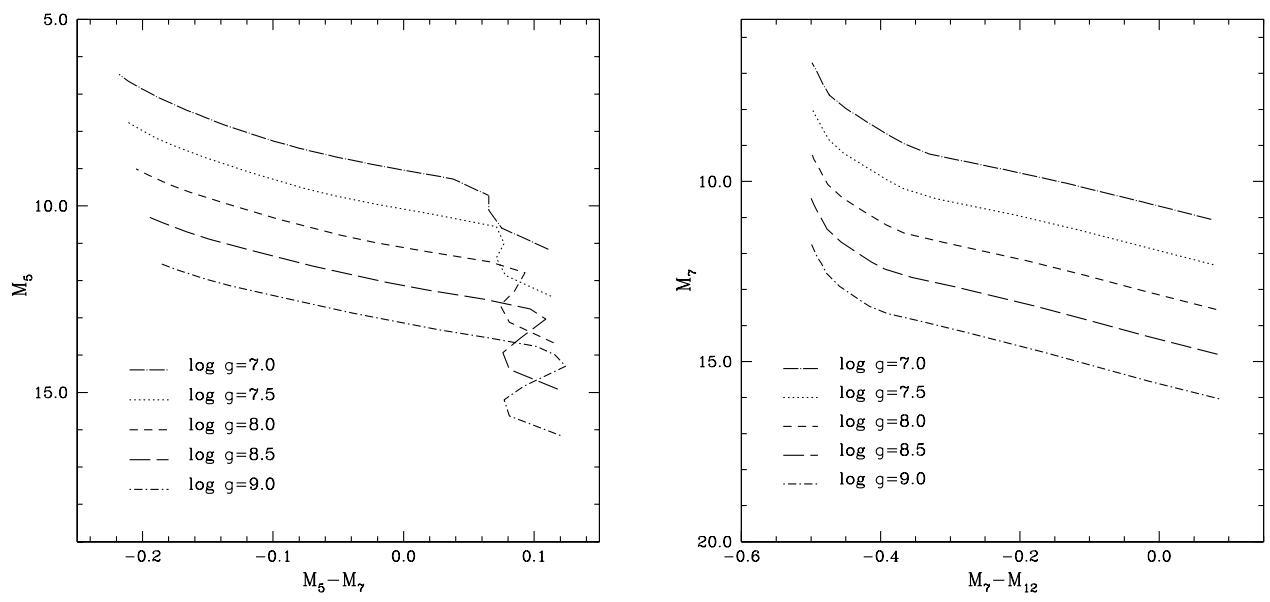

Figure 6.12: Color-magnitude diagram for filters 5, 7 and 12.

coolest stars. The right panel illustrates the almost linear behaviour of the magnitude versus the color, except for the hottest stars. For these, the magnitude changes more abruptly with the color. It has to be taken into account that considering an absorption line may introduce important changes in the behaviour of the magnitude, since its width and depth change with the temperature and surface gravity.

It is also interesting to observe the relation between the temperature and the color. In Fig. 6.13 we show such a relation for colors $M_{1}-M_{5}$ (top left panel), $M_{5}-M_{11}$ (top right panel), $M_{5}-M_{7}$ (bottom left panel), and $M_{7}-M_{12}$ (bottom right panel). All panels show a rather obvious logarithmic dependence for hot white dwarfs, as it should be expected. For cool white dwarfs the dependence turns out to be less clear for the left panels - colors $M_{1}-M_{5}$ and $M_{5}-M_{7}$, respectively. It is interesting to realize that for the color $M_{7}-M_{12}$ (bottom right panel), the color is an excellent indicator of the temperature, since the dependence on the surface gravity is extraordinarily weak.

\subsubsection{Cool white dwarfs}

White dwarfs have physical characteristics which are very different from those of main sequence stars and subdwarfs. As an example, and from the observational point of view, it is well known that white dwarfs and main sequence stars can have similar visual magnitudes, depending on the filter used, but very different power spectral densities. This is, thus, one of the reasons why it is so important to adopt a set of criteria when using only photometric data for distinguishing white dwarfs from main sequence stars.

In Kilic et al. (2004) the narrow-band filter DD051 was used for the photomet- 

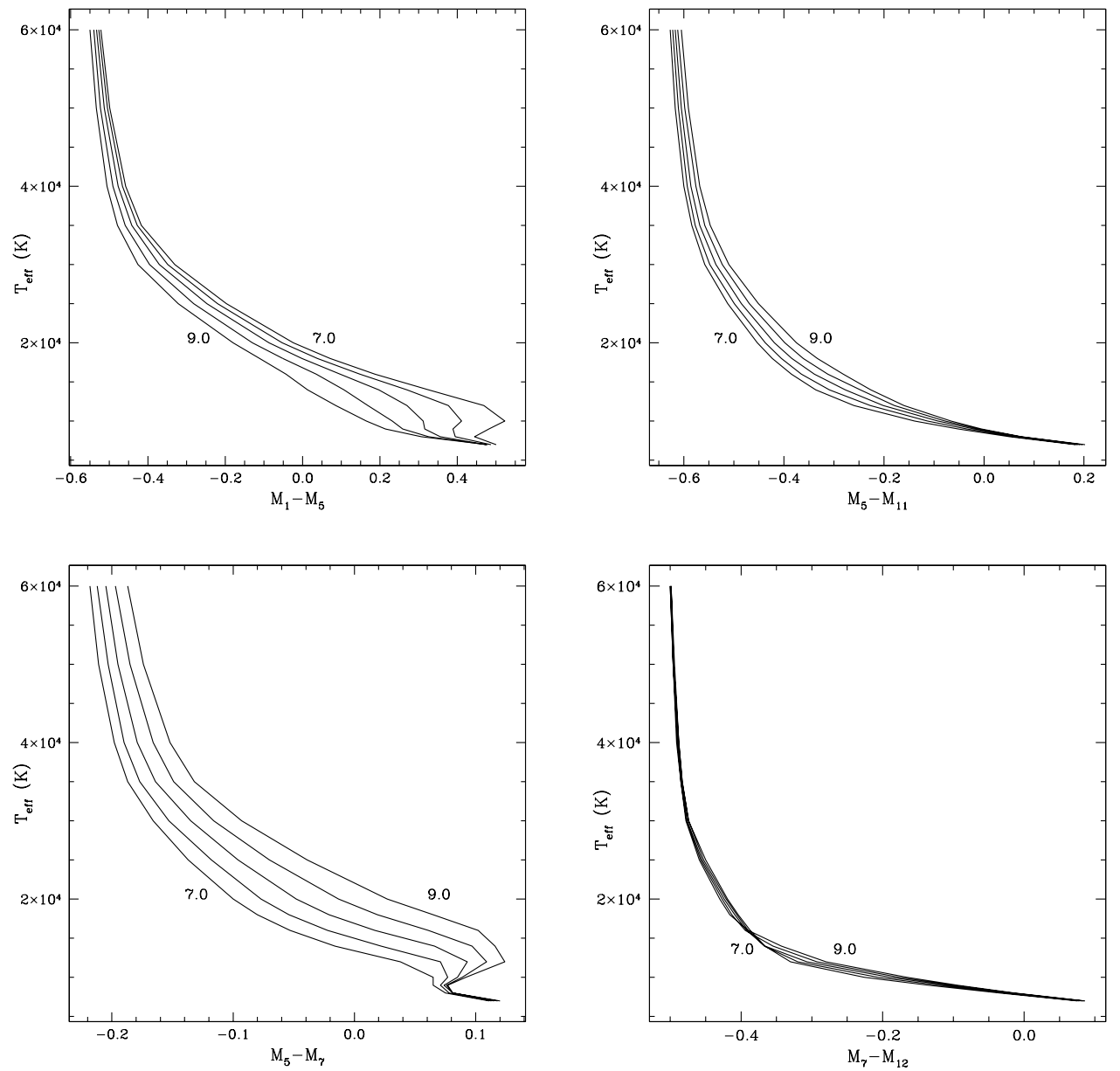

Figure 6.13: Temperature versus colors $M_{1}-M_{5}, M_{5}-M_{11}, M_{5}-M_{7}$ and $M_{7}-M_{12}$.

ric identification of cool white dwarfs. This filter is centered in $5150 \AA$, where the sudwarfs and main sequence stars show significant absorption from the Mgb triplet and/or MgH. Consequently, a color-color diagram using the Alhambra filter number 6 - which is the one centered in the same wavelength as the filter DD051 - should be useful to this regard, since white dwarfs would eventually be located at quite different positions from those of main sequence stars. Hence, this color-color diagram would potentially be very effective in recovering white dwarfs using only photometric data. In fact, Kilic et al. (2004) found that they were succesful to reject $\sim 65 \%$ of main sequence stars with broad-band colors similar to those of cool white dwarfs.

To explore such possibility we use the observational spectra of main sequence stars from the Pickles database and the theoretical spectra of DA white dwarfs 


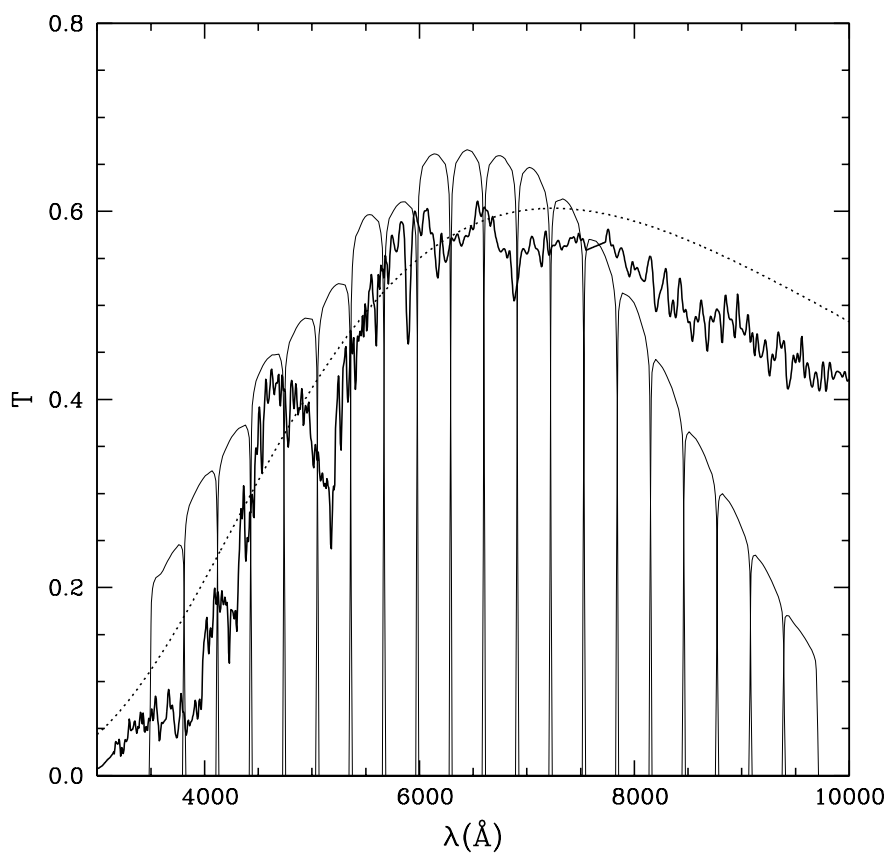

Figure 6.14: Spectra of a main sequence star from the Pickles database - thick solid line and of a blackbody of $4000 \mathrm{~K}$ - dotted line. The transmissions of the filters of the Alhambra survey — thin solid lines - are also plotted for the sake of identifying the relevant spectral features.

of the Koester database, as it has been done previously. However, the Koester database only covers the effective temperature range of hot and moderately cool white dwarfs, and the theoretical spectra for very cool white dwarfs - which for cool enough white dwarfs are featureless - are not available. Consequently, in order to model the behavior of very cool white dwarfs we have used blackbody spectra for temperatures between $60000 \mathrm{~K}$ and $3000 \mathrm{~K}$. In Fig. 6.14 a typical spectrum of a main sequence star from the Pickles database and the spectrum of a blackbody of $4000 \mathrm{~K}$ are represented. The transmission of the Alhambra filters is also plotted in order to see what kind of features are we taking into account in each of the filters. The $\mathrm{Mg}$ absorption line falls in the wavelength region of the filter number 6 .

In Fig. 6.15, we show the colors obtained with filters 6, 9 and 18 for white dwarfs, main sequence stars and a blackbody. The selection of the filters has been done considering those ones used in Kilic et al. (2004). Unfortunately, as it can be seen in this figure, using this set of filters we cannot distinguish main sequence stars and white dwarfs. Hence, we tried other possibilities and we found that the one which allows us to distinguish best between white dwarfs and main sequence stars 


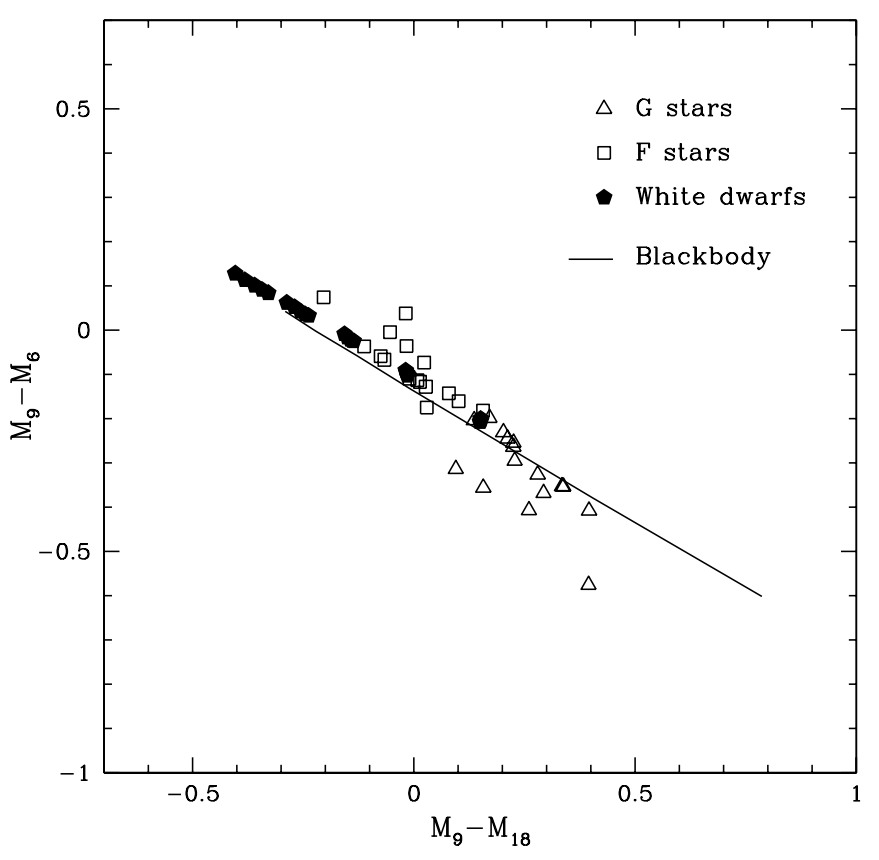

Figure 6.15: Color-color diagram for white dwarfs (solid pentagons), main-sequence stars (squares and triangles) and a blackbody (solid line) using filters 6, 9 and 18.

is the color-color diagram shown in Fig. 6.16, in which we take into account the flux accumulated in the filters 1, 6 and 11 . We see in this figure that both main-sequence stars and white dwarfs differ from the behaviour of a blackbody, as already noted by Kilic et al. (2004). This diagram will be very helpful to identify white dwarfs and in this way, decrease the number of white dwarfs needed to be spectroscopically confirmed.

\subsection{Number of white dwarfs in the Alhambra survey}

Taking into account the value of the limiting visual magnitude $(V=25.5)$ of the Alhambra survey and the location of the eight fields of 1 squared degree that this project proposes to cover, an estimate of the number of white dwarfs which will be detected can be done. In order to do so we need to use the white dwarf luminosity function. We remind the reader that the white dwarf luminosity function is defined as the number of white dwarfs per unit volume and per unit bolometric magnitude, and can be computed as follows: 


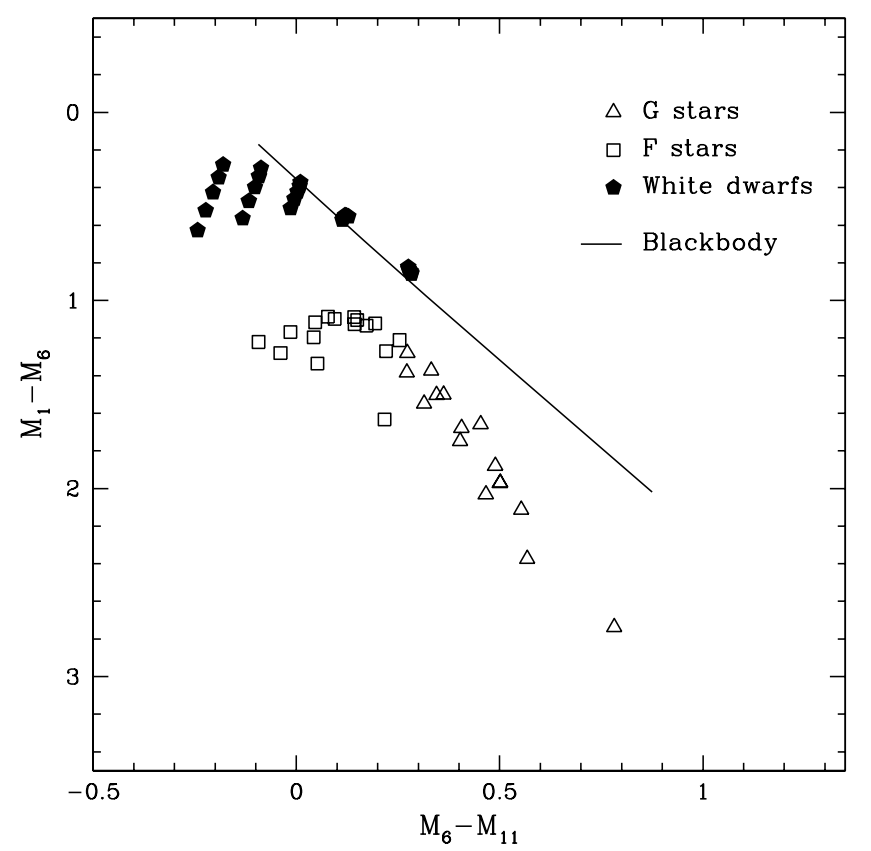

Figure 6.16: Color-color diagram for white dwarfs (solid pentagons), main-sequence stars (squares and triangles) and a blackbody (solid line) using filters 1, 6 and 11.

$$
n\left(M_{\mathrm{bol}}, T\right)=\int_{M_{\mathrm{i}}}^{M_{\mathrm{s}}} \Phi(M) \Psi\left(T-t_{\text {cool }}-t_{\text {prog }}\right) \tau_{\text {cool }} d M
$$

where $M$ is the mass of the parent star, $\tau_{\text {cool }}=d t / d M_{\mathrm{bol}}$ is the characteristic cooling time, $M_{\mathrm{s}}$ and $M_{\mathrm{i}}$ are the maximum and the minimum masses of the main-sequence stars able to produce a white dwarf of magnitude $M_{\text {bol }}$ at time $T, t_{\text {cool }}$ is the time necessary to cool down to this magnitude, $t_{\text {prog }}$ is the progenitor lifetime, and $T$ is the age of the population under study (disk or halo). Providing a relationship between the mass of the white dwarf and the mass of its progenitor, appropriate cooling sequences (Salaris et al. 2000) and the relationship between the mass of the progenitor and its main-sequence lifetime, Eq. (6.6) can be easily solved.

Taking into account the luminosity functions obtained in García-Berro \& Torres (1997), García-Berro et al. (1999) and Isern et al. (1998), García-Berro et al. (2004) for the disk and the halo populations, respectively, the number of white dwarfs for each of the Alhambra fields - see Fig. 6.1 - can be obtained. A total number of 300 white dwarfs is expected to be detected in the Alhambra survey. In Table 6.3 we detail the number of white dwarfs that will be detected in each one of the fields, for both the galactic disk and the halo white dwarf populations. As can be seen in each 
Table 6.3: Expected number of disk and halo white dwarfs for each of the Alhambra fields.

\begin{tabular}{lcc}
\hline \hline Fields & Disk & Halo \\
\hline ALHAMBRA-1 & $33 \pm 4$ & $1 \pm 1$ \\
ALHAMBRA-2 & $30 \pm 4$ & $1 \pm 1$ \\
ALHAMBRA-3 & $30 \pm 4$ & $2 \pm 1$ \\
COSMOS & $36 \pm 4$ & $2 \pm 1$ \\
HDF-N & $31 \pm 4$ & $1 \pm 1$ \\
GROTH & $32 \pm 4$ & $1 \pm 1$ \\
ELAIS-N1 & $37 \pm 4$ & $2 \pm 1$ \\
ALHAMBRA-8 & $33 \pm 4$ & $2 \pm 1$ \\
\hline \hline
\end{tabular}

of the fields we expect to find $\sim 30$ disk white dwarfs and $\sim 2$ halo white dwarfs. Hence, the Alhambra survey will notably increase the number of white dwarfs with accurate photometry, both for the disk and the halo population. Particularly, it should be taken into account that the actual number of spectroscopically identified white dwarfs is of the order of 2500 . Of these, only $\sim 300$ white dwarfs have accurate parallaxes and, thus, can be used to build the observational white dwarf luminosity function. The situation is even worst for the halo white dwarf population, since, despite its potential importance, only $\sim 2$ possible halo white dwarfs are presently known. These facts highlight the importance of surveys with characteristics similar to the Alhambra survey.

\subsection{Current status of the project}

A comparison of our predictions with observational data will be done as soon as these data become available. At the present moment, one of the eight fields of the Alhambra survey has been observed with the 20 filters. These data are still under reduction by the core team. 



\section{Chapter 7}

\section{Summary and conclusions}

The study of the white dwarf population is of paramount importance to unveil the history of our Galaxy. About 95\% of main sequence stars will end their evolutionary pathways as white dwarfs, and thus, the study of the white dwarf population gives details about the late stages of the life of the vast majority of stars. The evolution of white dwarfs is driven by a slow cooling process that lasts several Gyr (Isern et al. 1998). This means that these objects have imprinted a memory of the various episodes that the Galaxy has been subject to over its history, constituting useful objects to probe its structure and evolution. Moreover, it is thought that white dwarfs could also contribute significantly to the baryonic dark matter (García-Berro et al. 2004). For all these reasons, it is essential to improve our knowledge about the white dwarf population from all the possible points of view. In this thesis we have focused in improving one of the most important parameters related to white dwarfs, the initial-final mass relationship. This function is a key ingredient to many important aspects of astronomy: the determination of the ages of globular clusters and their distances, the study of the chemical evolution of galaxies, the determination of the rate of core collapse supernovae, to understand the star formation and feedback processes in galaxies, and for studying the properties of the galactic populations of field and cluster white dwarfs.

In chapter 1 we have given a brief summary of the main results that the theory of stellar evolution yields for low- and intermediate-mass stars, giving special emphasis to the last stages of the evolution of these stars. We have also briefly commented on the presently unsolved problems which bear importance with the observational determination of the initial-final mass relationship. These are metallicity, rotation, mass-loss processes and magnetism. Throughout this thesis we have tried to provide some clues on the dependence of the initial-final mass relationship on some of these parameters, and especially on the dependence on metallicity.

In chapter 2 we have studied a sample of common proper motion pairs comprised of a white dwarf and a FGK star. We have performed high signal-to-noise low res- 
olution spectroscopy of the white dwarf members, which led us to carry out a full analysis of their spectra and to make a re-classification when necessary. From the fit of their spectra to white dwarf models we have obtained their atmospheric parameters. Then, considering different cooling sequences - namely those of Salaris et al. (2000) and Fontaine et al. (2001) — their masses and cooling times were derived. Simultaneously, we have performed independent high resolution spectroscopic observations of their companions. Using the available photometry we have obtained their effective temperatures. Then, from a detailed analysis of their spectra and using either isochrones or their X-ray luminosity we have derived their metallicities and ages (i.e., the metallicities of the progenitors of the white dwarfs and their total ages), and from these the initial masses using stellar tracks. We have shown that common proper motion pairs containing white dwarfs can be useful to improve the initial-final mass relationship, since they cover a wide range of ages, masses and metallicities. The most relevant contribution of this study is the coverage of the range of initial masses corresponding to masses between 1.5 and $2 M_{\odot}$, which was not covered by the published work based on open cluster data (Ferrario et al. 2005; Dobbie et al. 2006) previously to the realization of this thesis. The coverage of the low-mass end of the initial-final mass relationship guarantees, according to stellar evolution, the study of white dwarfs with masses near the typical values, $M \sim 0.57 M_{\odot}$, which represent about $90 \%$ of the white dwarf population (Kepler et al. 2007). This is important because with this work we have increased the statistical value of the semi-empirical initial-final mass relationship. White dwarfs belonging to stellar clusters are usually more massive and thus, less representative of the Galactic white dwarf population. The recent work of Kalirai et al. (2007b) based on white dwarfs in old open clusters has also contributed to the low-mass range. The main differences of both studies are the distances and magnitudes of the white dwarfs studied. While the work of Kalirai et al. (2007b) is based on white dwarfs that belong to open clusters at distances larger than $2 \mathrm{kpc}$, the common proper motion pairs studied in this thesis are much closer $(d<100 \mathrm{pc})$, which allows a better spectroscopic study of the white dwarf member without the necessity of taking into account the effect of extinction, excluding in this way a possible systematic source of error. It is worth mentioning that in the case of common proper motion pairs, the accuracy in the total ages depends almost exclusively on the evolutionary state of the low-mass companions. Such relative accuracy becomes poor when the star is close to the ZAMS if the chosen method to derive the age is stellar isochrones. However, this can be improved if the star presents X-ray emission, since we have proved that the age can be derived with accuracy if the relation derived by Ribas et al. (2007) is used. In any case, the limitation in the age may not be critical to many common proper motion pairs. Planned deep surveys like GAIA, LSST or the Alhambra Survey will discover thousands of new white dwarfs, some of them belonging to wide binaries of this type. This will, undoubtely, improve the quality of our determination.

One of the studied common proper motion pairs turned out to be an extremely 
interesting system. We have applied the same method described in chapter 2 to derive their stellar properties, but we found a complete disagreement between the age of the companion and the cooling time of the white dwarf. In chapter 3 we have used the results of our analysis and have evaluated all the available literature regarding these objects to try to unveil the age and composition of the white dwarf member. We have carried out a thorough comparison of the kinematic properties of both objects with different works in the literature, favoring the scenario in which this common proper motion pair is indeed a former member of the Hyades cluster, and thus its members should have a coeval age of $\sim 0.7$ Gyr. This age is also in good accord with the value derived from the Li abundance obtained by Barrado y Navascués \& Stauffer (1996) for $\mathrm{BD}+26$ 730. We have evaluated different compositions for WD0433+270, but the properties of this white dwarf are only compatible with the $\mathrm{C} / \mathrm{O}$ and Fe-core cooling sequences. In the case of $\mathrm{C} / \mathrm{O}$-core, the cooling time obtained is of about 4 Gyr, which is more than 6 times the age of the Hyades open cluster. On the contrary, in the case of a Fe core, the associated cooling time is of $\sim 1$ Gyr. So, in order to be compatible with the age of the Hyades cluster, WD0433+270 should have an Fe core. However, there is still the possibility, although unlikely, that this pair is linked to the Hyades stream instead of the Hyades open cluster. This will relax the requirement of an exotic composition for the white dwarf core, since Chereul et al. (1999) pointed out that the oldest stars in this stream have an age of 2 Gyr. However, the existence of a system with an age of $\sim 4$ Gyr would have interesting consequences for Galactic dynamics, since it indicates the existence of an even older population in the Hyades stream. Thus, this would lend strong support to the model of a resonant origin for the Hyades stream (Famaey et al. 2007). Another implication of such an old age is the conflict with Li detection, which would imply a much lower destruction rate than expected. For these reasons we favor the scenario of WD0433+270 being a Fe-core white dwarf. The existence of white dwarfs with such core compositions could have important consequences for white dwarf formation models and also for the theories of core collapse and explosion of degenerate nuclei (Isern et al. 1991).

In chapter 4 we have revisited the initial-final mass relationship and discussed it in a more comprehensive manner. With this purpose, we have re-evaluated the available data in the literature, mainly based on open clusters, that are currently being used to define the semi-empirical initial-final mass relationship. We have used the atmospheric parameters, total ages and metallicities reported in the literature to derive the initial and final masses of these white dwarfs. Thanks to these data and our own work based on common proper motion pairs we have been able to collect a very heterogeneous sample of white dwarfs, covering a wide range of ages and metallicites. However, no clear dependence of the semi-empirical initial-final mass relationship on metallicity has been found in this study, presumably due to the large error bars of the observational data. Taking into account the procedure adopted in other recent works (Williams 2007; Ferrario et al. 2005) and, since there was no compelling reason to justify the use of a more sophisticated relationship, we have 
performed a weighted linear least-squares fit of these data. Taking into account the change of slope in the distribution around $2.7 M_{\odot}$ we believe that an independent fit of each region (above and below this point) should better represent the distribution. In order to quantify the correlation between the differences in final masses (observed and predicted by the linear equation) and metallicity, we computed the Spearman rank correlation coefficient and a bootstraping to quantify its errors. The coefficient obtained, $0.002 \pm 0.128$, confirms that these parameters are not correlated. This is not what is expected from the point of view of stellar evolution since mass-loss processes that take place during the RGB and the AGB phases are considered to be dependent on metallicity (Domínguez et al. 1999; Marigo 2001). As discussed in chapter 4, for each cluster the results present an intrinsic mass spread, which may indicate that mass loss could depend more on individual stellar properties than on a global mechanism. It has been also suspected that mass-loss processes could even be a stochastic phenomenon (Reid 1996), being thus impossible to reproduce them with models. Another explanation for the spread of masses found for each cluster could lie on the fact that we do not know the internal composition of the white dwarfs in our sample, and we are using cooling sequences that have fixed values for the $\mathrm{C} / \mathrm{O}$ ratio at the core. Most importantly we have used cooling sequences with fixed thicknesses of the $\mathrm{H}$ and He envelopes which may not be appropriate to describe the cooling of individual white dwarfs. This fact could affect the cooling times derived in approximately 1 Gyr at very low luminosities (Prada Moroni \& Straniero 2002). Moreover, according to Bergeron et al. (1992), atmospheres of DA stars below $12000 \mathrm{~K}$ could well be enriched in helium, while retaining their DA spectral type. This helium is brought to the surface when a significant hydrogen convection zone develops. Depending on the efficiency of convection the star could preserve the appearance of the Balmer lines, instead of turning into a non-DA white dwarf. Thus, the assumption of an unrealistic chemical compositon of the surface layers could have a large impact on the cooling times estimated, mainly at low effective temperatures.

Among the white dwarfs in common proper motion pairs that we have studied, we have found two stars that might represent the first evidence of the effect that rotation has in the evolution of a main-sequence star. This is in good agreement with the study carried out in Domínguez et al. (1996) that predicts that a fast-rotator progenitor gives rise to a more massive white dwarf than the non-rotator progenitor counterpart due to the conservation of angular momentum. On the other hand, one of the stars in our sample is a magnetic white dwarf. However, this star has a mass which is about half of the mean mass of magnetic white dwarfs, which is $\sim 0.93 M_{\odot}$ according to Wickramasinghe \& Ferrario (2005). From the initial and final masses obtained in this case it is difficult to favor one of the two main hypothesis regarding this issue: whether magnetic white dwarfs are more massive because the progenitors were also more massive (without any dependence on the magnetic field), or on the opposite, magnetic white dwarfs are more massive because the magnetic field had an influence during its evolution, favoring the growth of the core. Spectropolarimetric 
observations of the current sample of white dwarfs used to define the semi-empirical initial-final mass relationship would be very useful to shed some light upon this subject.

In chapter 5 we have evaluated the influence of the semi-empirical initial-final mass relationship previously derived in chapter 4 in the white dwarf luminosity function and we have compared the results with those obtained for other well-known parametrizations of the initial-final mass relationship. For the sake of consistency, we have carried out these calculations using whenever possible the stellar tracks corresponding to the same set of stellar evolutionary data. In all cases, the results were similar, specially for the hot branch of the white dwarf luminosity function. The fit of the cut-off in the luminosity function, which is located at $\log \left(L / L_{\odot}\right) \simeq-4.5$, yielded the same age for the Galactic disk in each case, $11 \mathrm{Gyr}$ approximately, except when the relations of Wood (1992) were used, for which we obtained 10.5 Gyr. Using the accurate determination of the masses of white dwarfs belonging to the Palomar Green Survey carried out by Liebert et al. (2005a) we have computed the white dwarf luminosity function constraining the range of masses. This is the first time that such a study is carried out. From a comparison of the results we have obtained that the initial-final mass relationship of Girardi et al. (2002) and Hurley et al. (2000) are the ones that produce more massive white dwarfs, while the one of Domínguez et al. (1999) produces less white dwarfs, and the semi-empirical relationship derived in chapter 4 gives results between these two cases. We have also evaluated the influence of the star formation rate on the results, considering our semi-empirical initial-final mass relationship. Our results show that the production of massive white dwarfs is highly dependent on the assumed star formation rate, since in the case of an exponentially decreasing star formation rate the density of white dwarfs decreases considerably. Thus, given the presently available observational data, any attempt to discern which initial-final mass relationship fits better the data is not feasible, since other inputs, such as the star formation rate, have a large influence on the results. Better observational data are sorely needed to disentangle this issue. Nevertheless, we were able to safely discard the initial-final mass relationship of Wood (1992), because when this relationship is used the agreement with the observations is rather poor.

Finally, chapter 6 is devoted to an observational project: the Alhambra Survey. Nowadays, the available technological facilities have made possible the development of important new deep surveys that will help considerably to disentangle some of the most important questions about the formation of our Galaxy. There are as well several new space missions (GAIA, SIM, Kepler) and ground deep surveys (LSST, PAN-Starrs and the Alhambra Survey) that will provide very deep images of the sky. This will enable us to not only test the theories of galaxy formation but also will help in building a knowledge of the nature and origin of the universe itself. Thanks to the depth of these surveys, thousands of new white dwarfs will be detected, including cool and ultracool white dwarfs, increasing the statistical value of the white 
dwarf luminosity function and allowing the observation of the white dwarf population beyong the cutoff in luminosity (Liebert et al. 1988 and Oswalt et al. 1996). At the same time, some of these new white dwarfs might belong to wide binaries as the ones studied in chapter 2, which will help to improve the initial-final mass relationship by applying the method developed in this thesis.

We have used the photometric system of the Alhambra Survey and the synthetic spectra of D. Koester to build color-color diagrams from which a comparison with the observational values could help to identify the white dwarf candidates. Since the Alhambra Survey will provide magnitudes in 20 different filters in the visible, we have been able to obtain a high number of color-color diagrams using different combinations of magnitudes (from different filters). Those diagrams for which we have obtained a wide grid will be very useful once we have reduced images, since white dwarfs with different $T_{\text {eff }}$ and $\log g$ can be well differenciated. Other color-color diagrams might be particularly interesting. For example, the color-color diagram resulting from the combination of magnitudes from filters 1, 6 and 11 leave white dwarfs and main-sequence stars in two well-differenciated regions, decreasing in this way the number of white dwarf candidates that need to be confirmed through spectroscopic observations.

Finally, we have estimated the number of white dwarfs that might be found in the Alhambra Survey. Taking into account the luminosity functions obtained in GarcíaBerro \& Torres (1997), García-Berro et al. (1999) and Isern et al. (1998), GarcíaBerro et al. (2004) for the disk and the halo populations, respectively, we consider that a total of 300 white dwarfs are expected to be detected in the Alhambra survey. It is worth to emphasize the relevance of this number, since the actual number of spectroscopically identified white dwarfs is of the order of 2500 . Of these, only $\sim$ 300 have accurate parallaxes. So, the Alhambra survey will provide a high number of white dwarfs that will help to increase the statistical value of the white dwarf luminosity function, and also to improve the initial-final mass relationship, since some of these white dwarfs will belong to wide binaries of our interest. Detailed study of the current and future suitable common proper motion pairs, together with the open cluster data, should help to explain the scatter in the semi-empirical initial-final mass relationship and to discern whether this is a single-valued function. One of our immediate priorities is to further extend the sample of wide binaries valid for this study. We are working on the search for more wide binaries of our interest in the NLTT catalog (Gould \& Chaname 2004) and also in the LSPM-north catalog (Lépine \& Bongiorno 2007). We think that any step in this direction is of crucial importance and would have a strong impact on stellar astrophysics, since the initial-final mass relationship is used in many different areas, such as the chemical evolution of galaxies, the determination of supernova rates, and the modelling of the star formation and feedback processes in galaxies, among others. 


\section{Appendix A}

\section{White dwarf cooling sequences}

\section{A.1 Basic theory of cooling}

The most relevant points of the cooling process of a white dwarf can be summarized as follows. After integrating over the entire star and assuming that the release of nuclear energy is negligible, the energy balance can be written as:

$$
L+L_{\nu}=-\int_{0}^{M_{\mathrm{WD}}} C_{\mathrm{V}} \frac{d T}{d t} d m-\int_{0}^{M_{\mathrm{WD}}} T\left(\frac{\partial P}{\partial T}\right)_{V, X_{0}} \frac{d V}{d t} d m+\left(l_{\mathrm{s}}+e_{\mathrm{g}}\right) \dot{m}_{\mathrm{c}}
$$

where $L$ and $L_{\nu}$ are the photon and the neutrino luminosities, respectively. Neutrinos are dominant for luminosities larger than $10^{-1} L_{\odot}$, while for luminosities smaller than $L=10^{-2} L_{\odot}$ the contribution of thermal neutrinos and nuclear reactions are negligible. The first term of the right-hand side of Eq.(A.1) represents the well-known contribution of the heat capacity of the star to the total luminosity (Mestel 1952). The second term takes into account the net contribution of compressional work to the luminosity. Finally, the third term in the right-hand side represents the energy release associated to solidification, where $l_{\mathrm{S}}$ is the release of latent heat, $e_{\mathrm{g}}$ corresponds to the gravitational energy release associated with the chemical differentiation induced by phase separation (Isern et al. 1997) and $\dot{m}_{\mathrm{c}}$ is the rate at which the crystallization front moves outward. All the terms in the right-hand side of Eq.(A.1) depend on the detailed chemical composition of the white dwarf.

\section{A.2 Carbon-oxygen white dwarfs}

In this thesis we have used the cooling tracks of Salaris et al. (2000), which consider a carbon-oxygen white dwarf with a higher abundance of oxygen at the center of the core, and with a $\mathrm{H}$ thick envelope on top of a He buffer, $q(\mathrm{H})=M_{\mathrm{H}} / M=10^{-4}$ and $q(\mathrm{He})=M_{\mathrm{He}} / M=10^{-2}$. These improved cooling sequences are based on the 
Table A.1: Cooling ages for white dwarfs of different masses.

\begin{tabular}{ccccccc}
\hline \hline $\log \left(L / L_{\odot}\right)$ & \multicolumn{5}{c}{$t_{\text {cool }}(\mathrm{Gyrs})$} \\
\hline & $0.54 M_{\odot}$ & $0.61 M_{\odot}$ & $0.68 M_{\odot}$ & $0.77 M_{\odot}$ & $0.87 M_{\odot}$ & $1.00 M_{\odot}$ \\
\hline-2.0 & 0.15 & 0.16 & 0.17 & 0.17 & 0.20 & 0.23 \\
-3.0 & 0.68 & 0.73 & 0.79 & 0.86 & 1.01 & 1.50 \\
-3.5 & 1.40 & 1.56 & 1.80 & 2.26 & 2.88 & 3.52 \\
-4.0 & 3.65 & 4.20 & 4.85 & 5.63 & 6.16 & 6.27 \\
-4.5 & 10.00 & 11.32 & 12.23 & 12.90 & 13.07 & 11.67 \\
-5.0 & 14.24 & 15.48 & 16.11 & 16.49 & 15.89 & 14.46 \\
\hline \hline
\end{tabular}

evolutionary code used by Salaris et al. (1997) but including an accurate treatment of the crystallization process of the carbon-oxygen core, considering phase separation upon crystallization, together with up-to-date input physics suitable for computing white dwarf evolution.

The chemical profiles of Salaris et al. (2000) consider carbon-oxygen white dwarfs in the mass range from 0.5 to $1.0 M_{\odot}$ and take into account the presence of large abundances of oxygen in the central regions due to the high rates of the ${ }^{12} \mathrm{C}(\alpha, \gamma){ }^{16} \mathrm{O}$ reaction. The high oxygen abundances in the central regions of the white dwarf minimize the effects of chemical differentiation upon crystallization as compared with the models in which a homogeneous 50/50 carbon and oxygen distributions throughout the star are adopted. For the sake of comparison, Salaris et al. (1997) also considered a lower rate for ${ }^{12} \mathrm{C}(\alpha, \gamma){ }^{16} \mathrm{O}$ reaction, which gives a smaller oxygen content at the center of the white dwarf. This leads to a larger heat capacity and therefore to a slower cooling rate. For example, the central value of the oxygen mass fraction in a white dwarf with a total mass of $0.61 M_{\odot}$ is $X_{\mathrm{O}}=0.74$ for the high rate and $X_{\mathrm{O}}=0.57$ for the low rate. In Salaris et al. (2000) they finally adopt the high rate for calculating the cooling sequences, although they remark that the cooling times obtained depend strongly on the effective cross section of the ${ }^{12} \mathrm{C}(\alpha, \gamma){ }^{16} \mathrm{O}$ reaction used.

The equation of state for the carbon-oxygen binary mixture in the gaseous phase was taken from Straniero (1988), while for the liquid and solid phases the detailed equation of state from Segretain et al. (1994) was used. At low luminosities, the evolution of the cooling process is dominated by the release of latent heat upon crystallization, together with the energy released by chemical fractionation. In this models, the associated release of latent heat was self-consistently computed and turned out to be $0.77 k_{\mathrm{B}} T$ per ion. The additional energy release due to phase separation of the carbon-oxygen mixture upon crystallization was computed following closely Isern et al. (1997) and Isern et al. (2000). The results obtained by Salaris 


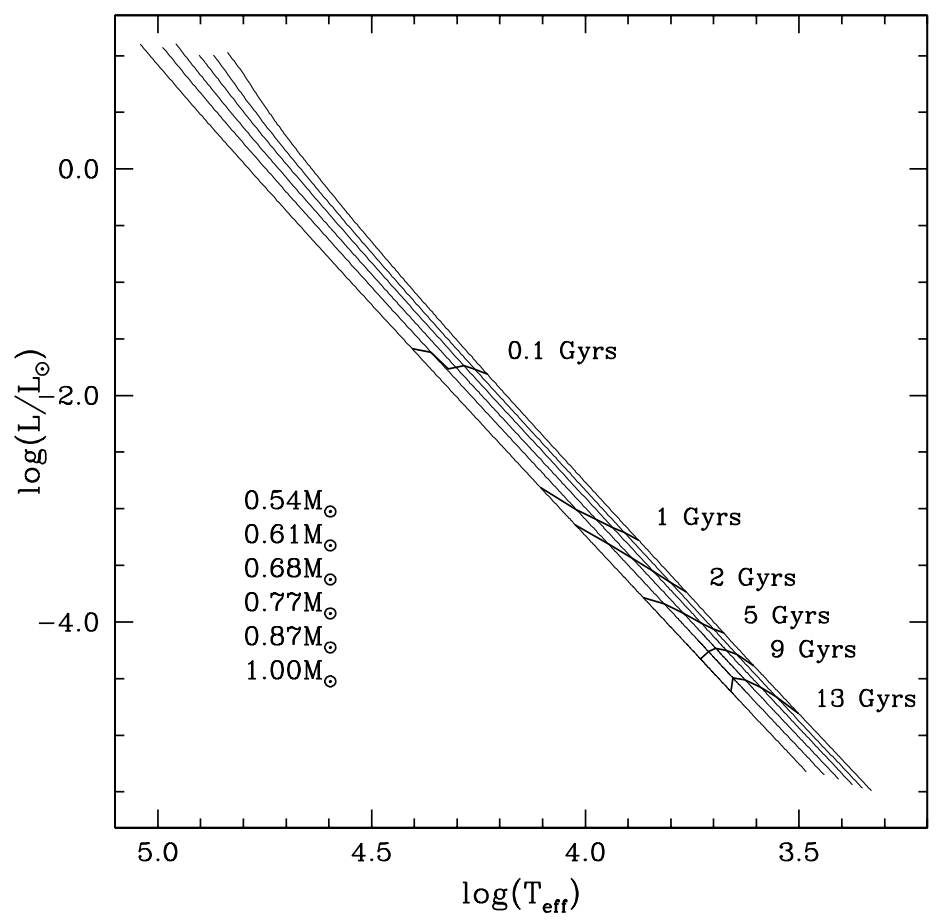

Figure A.1: Luminosity versus effective temperature diagram for different white dwarf masses.

et al. (1997) indicate that the lowest central chemical abundance of oxygen occurs for a $1.0 M_{\odot}$ white dwarf, thus enhancing the effect of phase separation. Moreover, the size of the central oxygen-rich region reaches its maximum for this mass enlarging the size of the mixing region and, consequently, also increasing the effect of phase separation. Exactly the opposite occurs for a $0.54 M_{\odot}$ white dwarf. For intermediate core masses the situation is more complex since the two previously mentioned effects are not linear and therefore the analysis of their combination is more difficult. A comparison of the cooling times obtained with and without considering phase separation is indicated in Salaris et al. (2000). This delay is maximum for the central range of white dwarf core masses (around $\sim 0.768 M_{\odot}$ ). Besides the core composition, it is worth to mention the importance of the outer layers of the white dwarf in the cooling process, since they control the power radiated away by the star, and hence, the cooling rate. As mentioned before, the adopted chemical structure of the outer layers in these models are the standard thick envelope of $\mathrm{H}$ and $\mathrm{He}$, with $q(\mathrm{H})=10^{-4}$ and $q(\mathrm{He})=10^{-2}$, respectively.

In Fig. A.1 we have represented the luminosity versus the effective temperature 


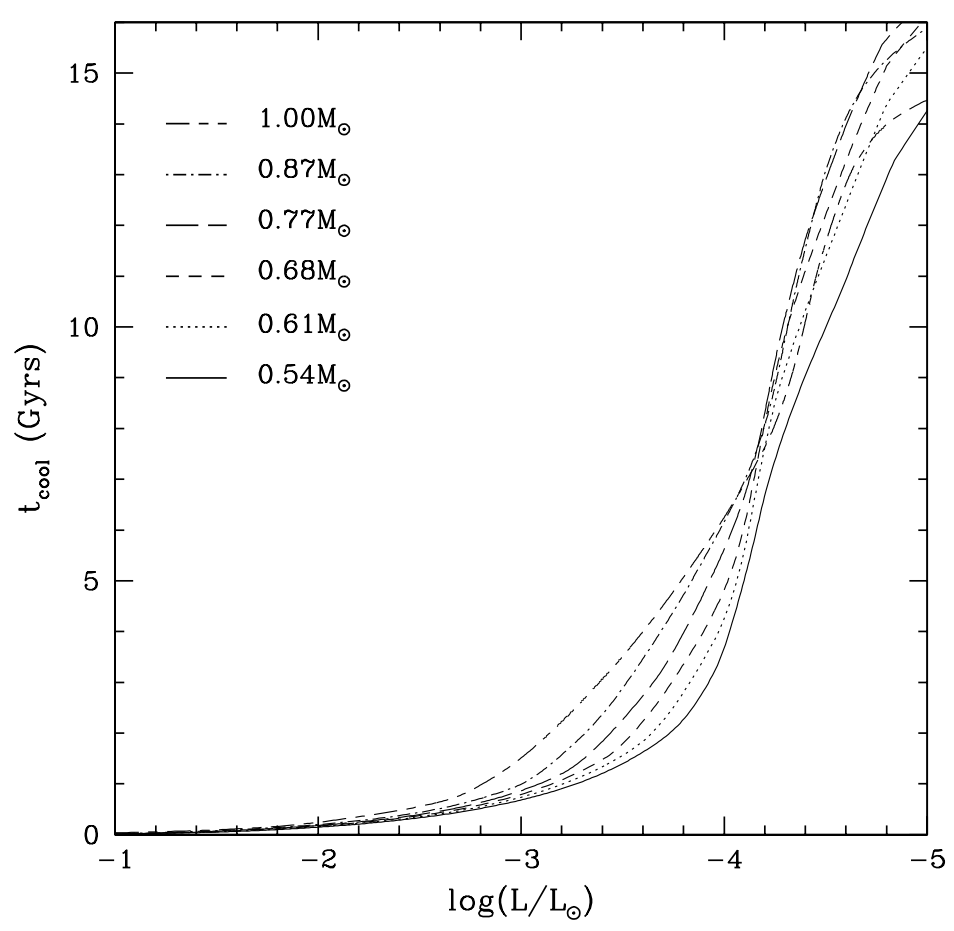

Figure A.2: Cooling curves for different white dwarf masses.

for different values of the white dwarf mass. The cooling isochrones are represented as thick solid lines. From this figure it can be seen that in general terms, a white dwarf with a small mass cools faster than a massive white dwarf. As an example, when the cooling time is 5 Gyrs, a white dwarf of $0.54 M_{\odot}$ has reached an effective temperature of $3400 \mathrm{~K}$ while a white dwarf of $1.00 M_{\odot}$ still has $5000 \mathrm{~K}$. However, by looking at the cooling times of Table A.1, it can be seen that at luminosities smaller than $\left.\log L / L_{\odot}\right)=-4.5$ the cooling rate of the massive stars increases and, as a result, the cooling time necessary to reach a $\log \left(L / L_{\odot}\right)=-5.0$ is approximately the same for a $0.54 M_{\odot}$ and for a $1.00 M_{\odot}$ white dwarf, while is longer for an intermediate mass white dwarf of mass $0.68 M_{\odot}$.

The cooling times as a function of the luminosity are shown in Fig. A.2. The onset of crystallization is clearly marked by the change in the slope of the cooling curves. Massive white dwarfs crystallize at higher temperatures (luminosities) because they have larger central densities and their oxygen abundance does not vary significantly.

In order to be able to check the sensitivity of our results to the adopted cooling tracks we have also used the cooling sequences of Fontaine et al. (2001) with different core compositions. For this purpose a set of standard thick-envelope DA stellar 


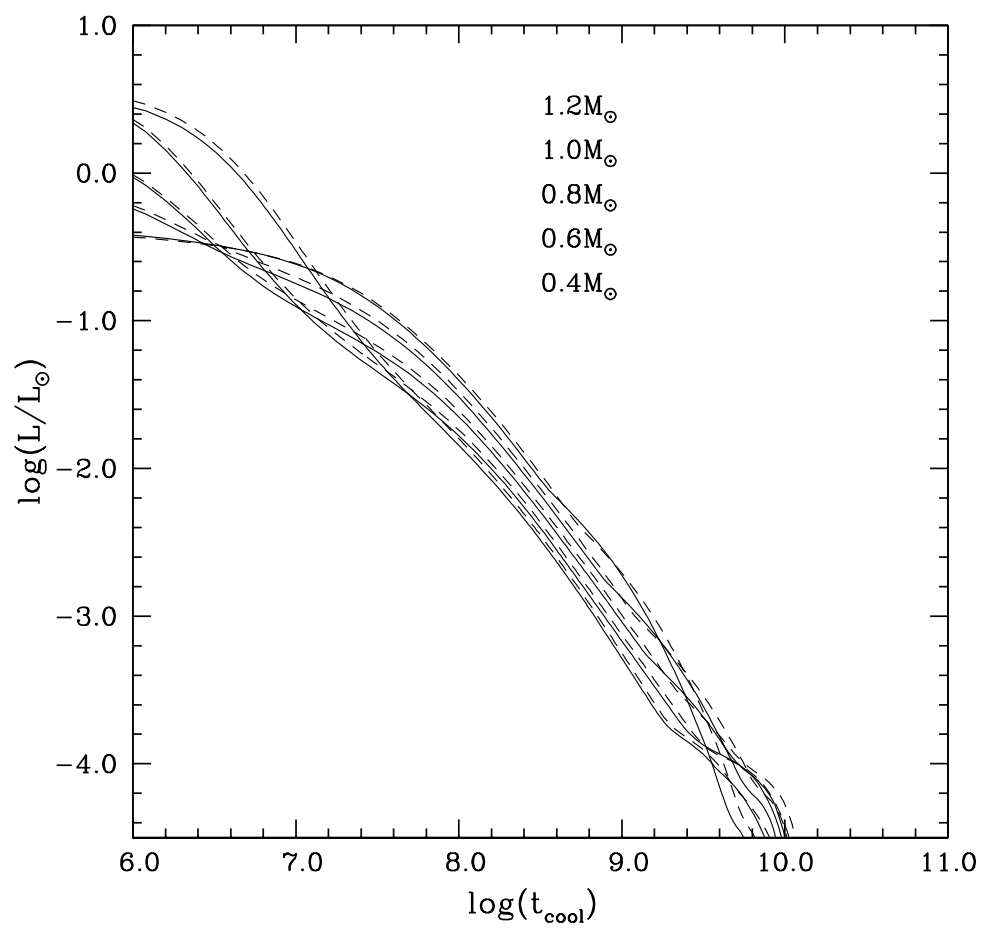

Figure A.3: Luminosity versus cooling time according to Fontaine et al. (2001) for different masses. The solid lines correspond to the models which consider a 50/50 carbon-oxygen white dwarf and the dashed lines to the ones that use a pure carbon core white dwarf.

models with pure carbon cores, a He mantle, $q(\mathrm{He})=10^{-2}$, and an outermost $\mathrm{H}$ layer, $q(\mathrm{H})=10^{-4}$, were adopted. In the second series of calculations carbon-oxygen cores with a composition of $50 / 50$ by mass with the same envelope characteristics were used. These cooling tracks have been calculated for 23 different masses in the mass interval ranging from 0.2 to $1.3 M_{\odot}$, with steps of $0.05 M_{\odot}$. The models with carbon-oxygen cores are more realistic since they include the retarding effect of element sedimentation when it is undergoing crystallization. The cooling time derived in this case remains smaller than that of a pure carbon-core white dwarf. Anyway, the initial choice of models consiting in a pure carbon core composition is interesting, since the ages obtained can be considered as upper limits.

This difference in the cooling between the carbon-oxygen models and the pure carbon ones is reflected in Fig. A.3, where it can be seen how a white dwarf with a pure carbon core cools slower than a carbon-oxygen white dwarf of the same mass. The larger heat capacity of carbon in comparison with that of oxygen implies that a larger amount of energy is necessary to change the temperature of the core. 


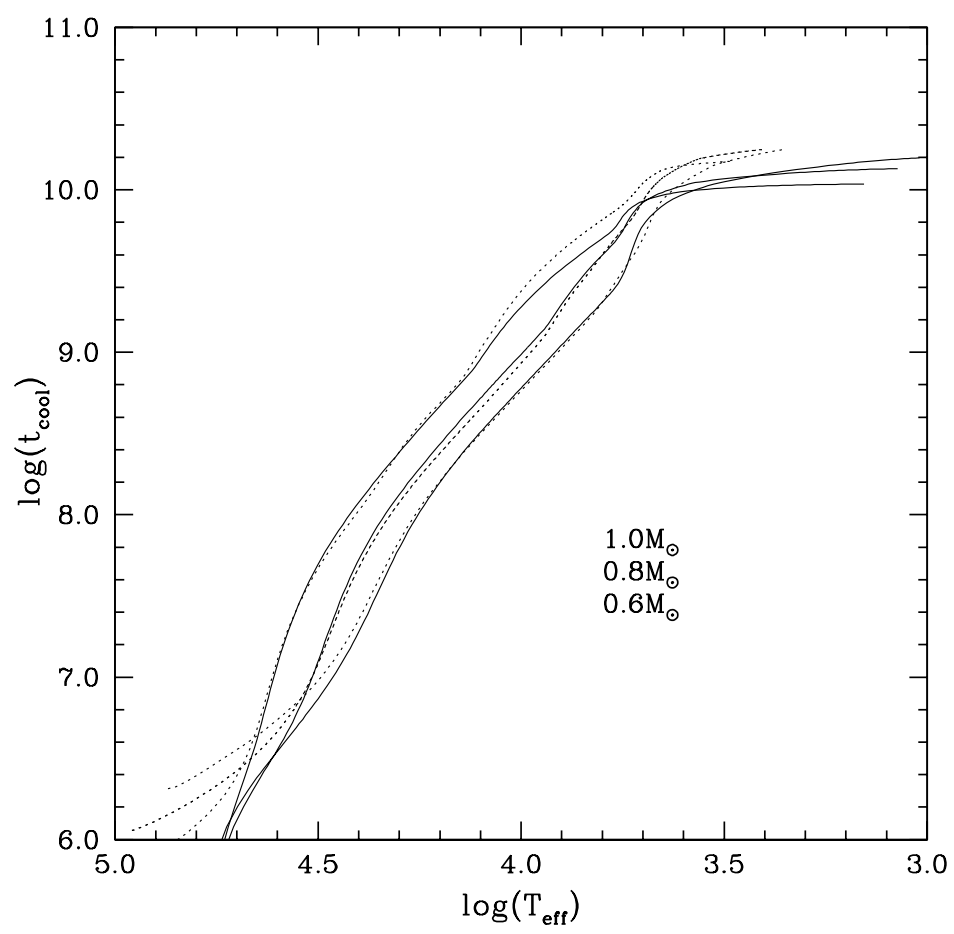

Figure A.4: Cooling curves of Fontaine et al. (2001) (solid lines) and of Salaris et al. (2000) (dotted lines) for the same white dwarf masses.

In Fig. A.4 we compare the carbon-oxygen models of Fontaine et al. (2001) with those of Salaris et al. (2000), which consider a white dwarf with a higher abundance of oxygen at the central regions of the core. It can be noticed that in terms of the cooling time the consideration of the first or the second model is not very determinant. However, it can be noted that in the model of Salaris et al. (2000) a white dwarf with a relatively high mass cools faster than in the model of Fontaine et al. (2001). Besides the different assumptions about the abundances in the core of the white dwarf, both models have more differences such as the adopted initial conditions. These particularities that characterize each model are explained in detail in Fontaine et al. (2001), and we refer the interested reader to this work for a detailed discussion about the origin of the differences.

\section{A.3 Oxygen-neon white dwarfs}

Massive white dwarfs have progenitors with masses $M \geq 8 M_{\odot}$ and are thought to be made of oxygen and neon. Although the evolution of isolated stars that produce 


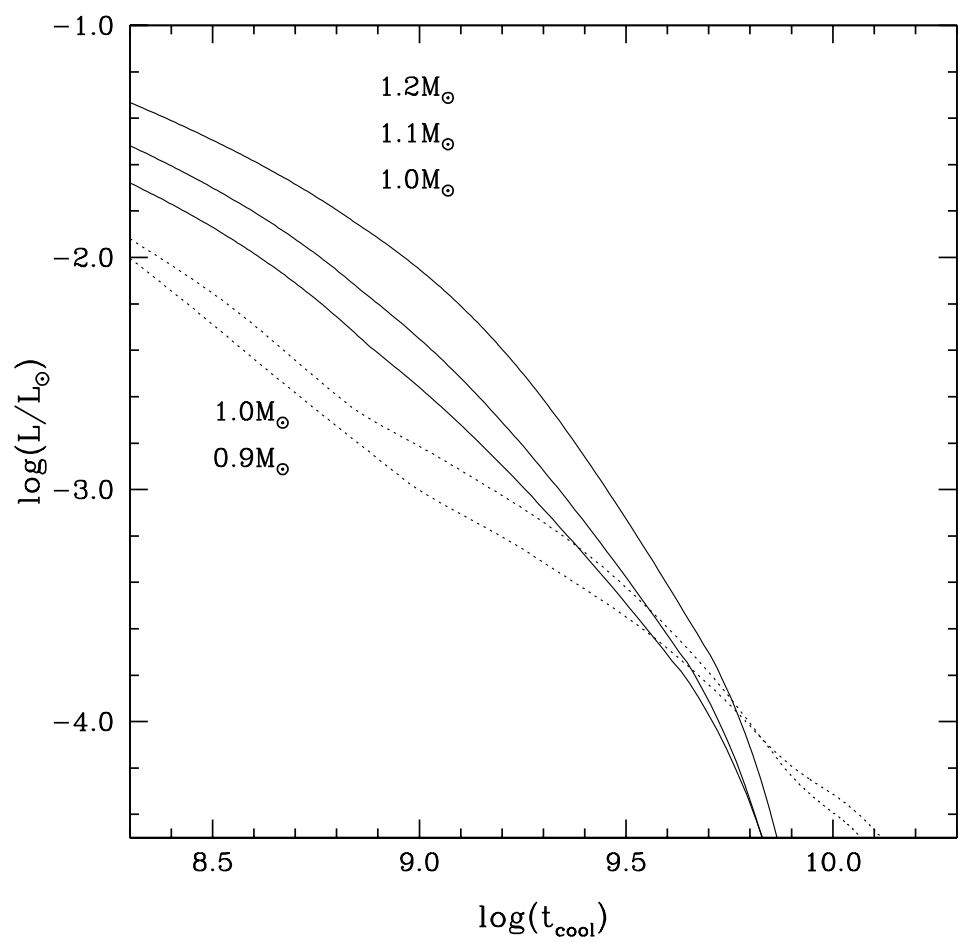

Figure A.5: Luminosity versus cooling time for oxygen-neon white dwarfs with different masses (solid lines) and for carbon-oxygen white dwarfs (dotted lines).

massive oxygen-neon white dwarfs is still not well explained and the expected number of these white dwarfs should be small, we take them into account when calculating the white dwarf luminosity function. It is worth mentioning as well that another suggested scenario for the production of oxygen-neon white dwarfs, apart from the evolution of isolated stars, is the merger of two white dwarfs in a close binary system (Gil-Pons \& García-Berro 2001), which can increase the number of white dwarfs of this type.

The cooling tracks for oxygen-neon white dwarfs used in this thesis are those of García et al. (1997). They computed cooling sequences for white dwarfs of masses 1.01, 1.1 and $1.2 M_{\odot}$ following the method described in Díaz-Pinto et al. (1994), where the cooling times are derived from the binding energy of the white dwarf and a relationship between the temperature of its core. They considered a purehelium envelope of thickness $q(\mathrm{He})=10^{-4}$. The equation of state used is that of Segretain et al. (1994) and the neutrino losses, which are important at relatively high temperatures, were also taken into account following the prescriptions of GarcíaBerro et al. (1996). 


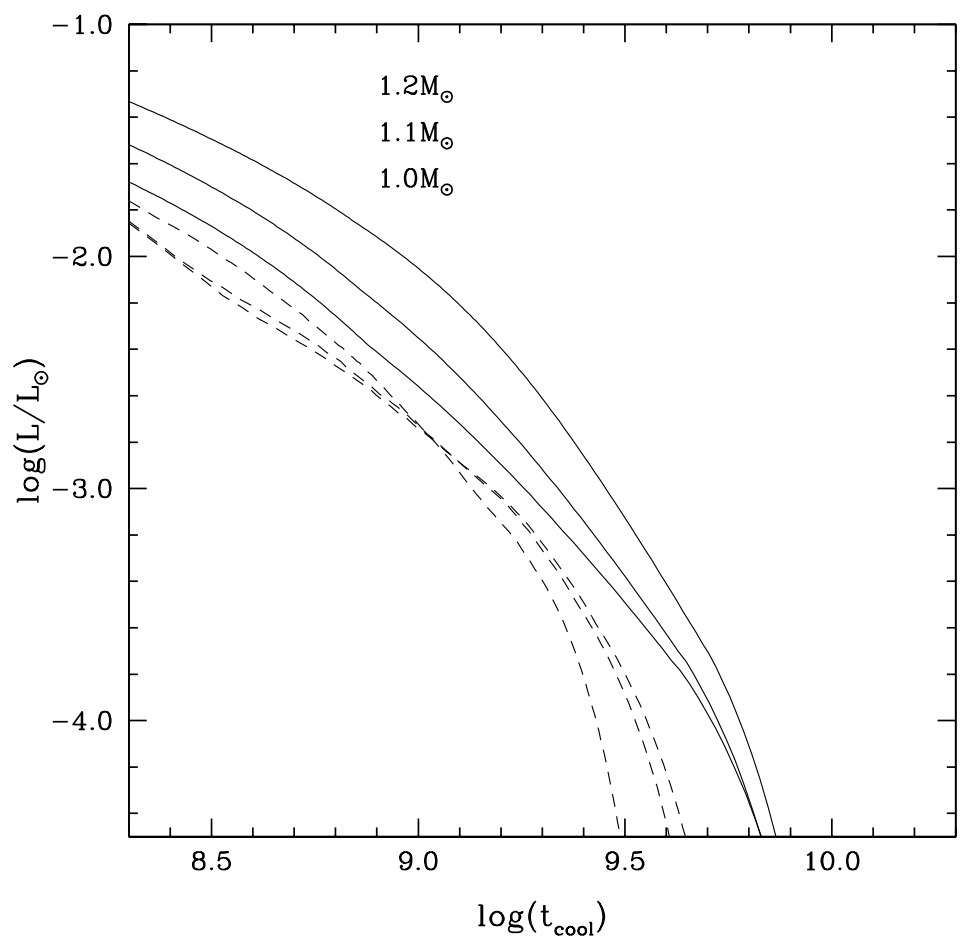

Figure A.6: Cooling curves for oxygen-neon white dwarfs of García et al. (1997) — solid lines - and the ones corresponding to their recent revision, Althaus et al. (2007) — dashed lines.

In Fig. A.5 we have plotted the cooling curves of García et al. (1997) for oxygenneon white dwarfs of different masses $\left(1.0,1.1\right.$ and $\left.1.2 M_{\odot}\right)$. The values of the cooling times necessary to reach a given luminosity are given in Table A.2. For the sake of comparison the cooling sequences for carbon-oxygen white dwarfs of masses 0.9 and $1.0 M_{\odot}$ are also represented in Fig. A.5(dotted lines). In general, it can be noted that for a given mass an oxygen-neon white dwarf cools faster than a carbon-oxygen white dwarf. This is due to the fact that the average charge of the oxygen-neon mixture is larger than that of the carbon-oxygen mixture and, consequently, the crystallization temperature is larger. Hence, the energy released by chemical sedimentation upon crystallization has smaller effects on the cooling times since this energy is released at larger luminosities. The onset of crystallization is clearly marked by a sudden change in the slope of the cooling curves of carbon-oxygen white dwarfs, while it is difficult to note it in the case of the oxygen-neon white dwarf cooling sequences. Moreover, in an oxygen-neon white dwarf there is no release of gravitational energy due to chemical differentiation since there is no difference between the chemical 
Table A.2: Cooling ages for oxygen-neon white dwarfs of different masses.

\begin{tabular}{cccc}
\hline \hline $\log \left(L / L_{\odot}\right)$ & \multicolumn{3}{c}{$t_{\mathrm{cool}}(\mathrm{Gyr})$} \\
\hline & $1.0 M_{\odot}$ & $1.1 M_{\odot}$ & $1.2 M_{\odot}$ \\
\hline-1.0 & 0.017 & 0.033 & 0.061 \\
-2.0 & 0.412 & 0.577 & 0.921 \\
-3.0 & 1.806 & 2.179 & 2.845 \\
-3.6 & 3.547 & 3.885 & 4.614 \\
-4.0 & 5.114 & 5.304 & 5.990 \\
-4.6 & 7.052 & 6.995 & 7.565 \\
\hline \hline
\end{tabular}

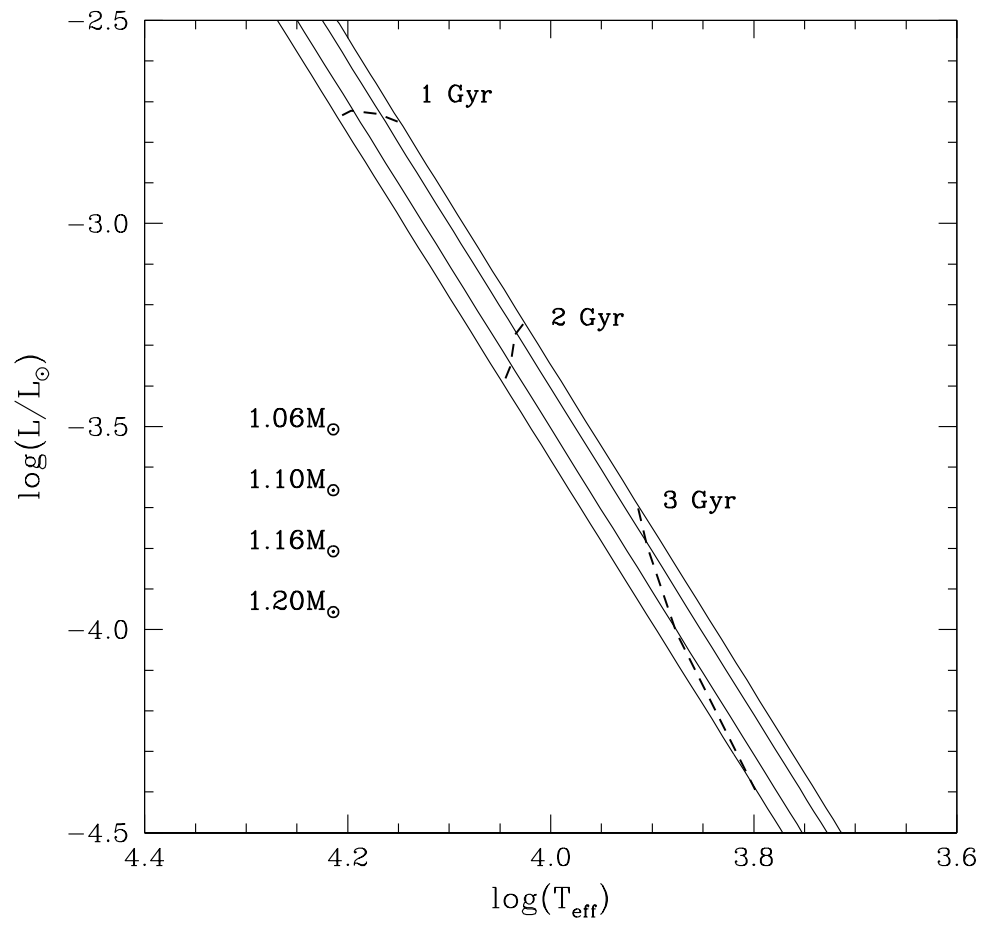

Figure A.7: Luminosity versus effective temperature diagram for the cooling sequences of Althaus et al. (2007) for different white dwarf masses. Thick solid lines represent cooling isochrones. 
compositions of the liquid and the solid phases. It also has to be taken into account that the oxygen-neon core has a reduced heat capacity due to the larger average atomic mass of the mixture and, therefore, the cooling time-scales are consequently shorter. The net result of all these effects is that oxygen-neon white dwarfs cool down much faster than carbon-oxygen white dwarfs of the same mass. To illustrate this we can consider the numbers given in Tables A.1 and A.2. A carbon-oxygen white dwarf needs about 9 Gyr to reach a luminosity of $\log \left(L / L_{\odot}\right)=-4.5$, whereas an oxygen-neon white dwarf only needs 7 Gyr to reach the same luminosity.

Recently, in Althaus et al. (2007), the authors have done a recalculation of these cooling sequences using updated physics suitable for this purpose. Actually, in Fig. A.6 we show the cooling curves for oxygen-neon corresponding to García et al. (1997) and the new ones (Althaus et al. 2007). As it can be noted, according to the new cooling sequences a white dwarf with a given mass cools faster than in the old models. In Fig. A.7 we show the luminosity versus effective temperature diagram of Althaus et al. (2007) for white dwarfs with an oxygen-neon core. The thick solid lines represent the cooling isochrones. 


\section{Appendix B}

\section{Stellar evolutionary tracks}

Throughout this thesis we have used the stellar evolutionary tracks of different authors, and their corresponding initial-final mass relationships, if available. In this appendix we give more details on each set of stellar models.

Domínguez et al. (1999) computed models covering the mass range from 1.2 to 9.0 $M_{\odot}$ and chemical compositions within the range of metallicities $0.0001 \leq Z \leq 0.02$ and helium abundances in the interval $0.23 \leq Y \leq 0.28$. The evolutionary tracks in the Hertzsprung-Russell diagram are reported in Fig. B.1. They considered the stellar evolution from the ZAMS up to the end of the early asymptotic giant branch (E-AGB) phase. The Padova group, namely Girardi et al. (2002), computed stellar evolutionary tracks and isochrones for low- and intermediate-mass stars from 0.15 to $7 M_{\odot}$, and from $Z=0.0004$ to 0.03 . These tracks are the corresponding ones to the initial-final mass relationship of Marigo (2001) and they cover the evolution from the ZAMS up to the TP-AGB phase or during C-ignition, depending on the mass. In Fig. B.1 we have also plotted the stellar evolutionary tracks of Girardi et al. (2002) as dotted lines. From a comparison of both stellar tracks it can be noted that essential differences in the evolution of stars with the same mass occur. For example, He ignition occurs at smaller effective temperatures in the case of Girardi et al. (2002), the luminosity is larger... These differences basically rely on the different treatment of the different physical processes involved in the calculations.

We have also taken into account the commonly used evolutive models of Hurley et al. (2000). However, these authors worked out a completely different approach to obtain stellar evolutionary tracks and isochrones. They fitted observational data from eclipsing binaries and open clusters and obtained analytic formulae for stellar evolution as a function of mass and metallicity. These formulae cover the range of masses from 0.5 to $50 M_{\odot}$ for seven values of metallicity, $Z=0.0001,0.0003,0.001$, $0.004,0.01,0.02$ and 0.03 .

It is important to distinguish here between the calculations of stellar evolutionary tracks and the initial-final mass relationship, since some of these groups have carried 


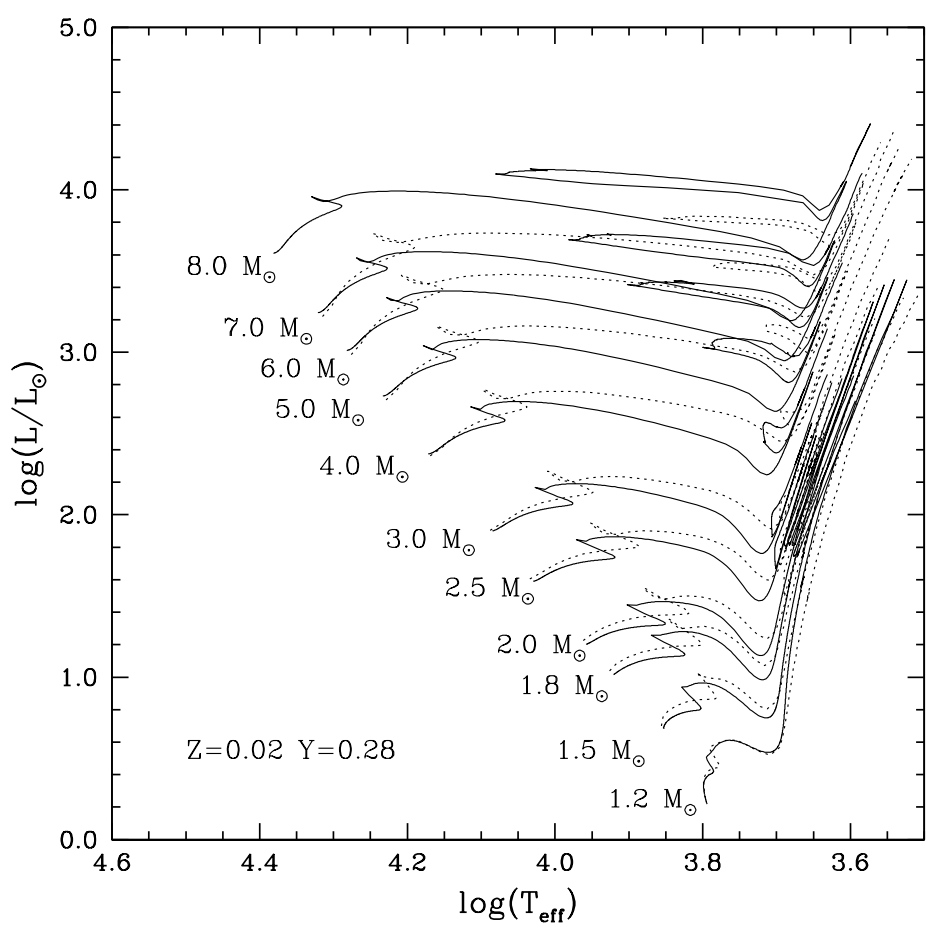

Figure B.1: Stellar tracks of Domínguez et al. (1999) — solid lines — and of Girardi et al. (2002) — dotted lines — for different initial masses.

out both computations. The stellar evolutionary tracks usually stop at a certain stage in the evolution of the star (usually at the first thermal pulse of the AGB phase), while the initial-final mass relationship is calculated extending this evolution until the formation of the white dwarf, considering the mass-loss processes.

\section{B.1 The treatment of convection}

When dealing with convective regions Domínguez et al. (1999) consider the Schwarzschild criterion for a chemically homogeneous fluid. This criterion is based on the evaluation of the expected temperature gradient produced by radiative transport of energy. When the required gradient is too steep, convection instead of radiative flux accounts for transport of energy. On the other hand, Girardi et al. (2002) describe the energy transport in the outer convection zone according to the mixing-length theory of Böhm-Vitense (1958). The location of convective boundaries is estimated by means of an algorithm which takes into account overshooting from the borders of both core and envelope convective zones. Mixing of material in the core of a given star significantly alters its lifetime, since it defines the value of $M_{\text {crit }}$, which is the max- 
imum initial mass of a star able to develop a degenerate $\mathrm{C} / \mathrm{O}$ core, and thus, able to reach the AGB phase. This critical mass largely depends on the previous evolutionary history of the star, mainly on the extension of the convective core during the H-burning phase. Evolutionary models that adopt the Schwarzschild criterion to define the convective boundaries predict larger values for $M_{\text {crit }}$ than those models that assume some overshoot beyond the last formally stable layer against convection. For instance, the value of $M_{\text {crit }}$ is between 7 and $8 M_{\odot}$ for Domínguez et al. (1999) and $5 M_{\odot}$ for Marigo (2001), although this value depends on metallicity. Thus, for a star with the same initial mass, the resulting white dwarf is more massive in the case in which the stellar evolutionary tracks of Marigo (2001) are considered than when the results of Domínguez et al. (1999) are adopted.

Some of the effects of including overshooting in the stellar models can be noted in Fig. B.1. During the core H-burning phase, the star has a larger He core, a larger luminosity, and a longer lifetime. During the following core He-burning phase, the luminosity is again larger and the lifetime is shorter than in the absence of overshooting. The models of the Padova group assume intermediate core overshoot $(\Lambda=0.5)$ and stronger envelope undershoot $(\Lambda=0.7)$. The parameter $\Lambda$ is the extension of this additional mixed region, and it is usually expressed as a fraction of the local pressure scale height crossed by the convective cells in the convectively stable region outside the Schwarschild convective boundary. In chapter 4, we took into account the stellar tracks of Pietrinferni et al. (2004) when calculating the initial masses of the white dwarfs belonging to M37. They carried out two sets of calculations with the same physical assumptions and inputs, but with and without considering overshooting. Thus, these evolutionary models let us evaluate precisely the effect of overshooting in the masses of the progenitors.

\section{B.2 Mass-loss processes}

Most stellar tracks do not include mass-loss during the thermally pulsing AGB phase. Thus, in this section we refer exclusively to the theoretical initial-final mass relationships. The final white dwarf mass will depend on how far the $\mathrm{C} / \mathrm{O}$ core can grow during this phase. Since the mass loss mechanisms are still not well understood from physical principles, different laws can be assumed to express these processes in the calculations. Domínguez et al. (1999) treat the mass-loss processes during the AGB phase using the classic approach. That is, they adopt a modified version of the mass-loss rate of Reimers (1975), which is based on an extrapolation of the treatment of mass loss in the RGB to the AGB, but using different values for the efficiency parameter $\eta$. On the other hand, Marigo (2001) uses a more modern approach that considers a semi-empirical formalism (Vassiliadis \& Wood 1993), which combines pulsational theories with observations of variable AGB stars. However, we stress that very little is known about the mass-loss rates during this phase. 



\section{Bibliography}

Affer, L., Micela, G., Morel, T., Sanz-Forcada, J. \& Favata, F., 2005. Spectroscopic determination of photospheric parameters and chemical abundances of 6 K-type stars^. A\&SA, 433, 647-658.

Allende Prieto, C., Barklem, P. S., Lambert, D. L. \& Cunha, K., 2004. $\mathrm{S}^{4} \mathrm{~N}$ : A spectroscopic survey of stars in the solar neighborhood. The Nearest 15 pc. $A \mathscr{E} A, \mathbf{4 2 0}, 183-205$.

Althaus, L. G., García-Berro, E., Isern, J. \& Córsico, A. H., 2005. Massradius relations for massive white dwarf stars. A\&A, 441, 689-694.

Althaus, L. G., García-Berro, E., Isern, J., Córsico, A. H. \& Rohrmann, R. D., 2007. The age and colors of massive white dwarf stars. A\&SA, 465, 249-255.

Aznar Cuadrado, R., Jordan, S., Napiwotzki, R., Schmid, H. M., Solanki, S. K. \& Mathys, G., 2004. Discovery of kilogauss magnetic fields in three DA white dwarfs. AEGA, 423, 1081-1094.

Barrado y Navascués, D., Stauffer, J. R., Bouvier, J. \& Martín, E., 2001. From the Top to the Bottom of the Main Sequence: A Complete Mass Function of the Young Open Cluster M35. ApJ, 555, 546.

Barrado y Navascués, D., Fernandez-Figueroa, M. J., Garcia Lopez, R. J., de Castro, E. \& Cornide, M., 1997. The age-mass relation for chromospherically active binaries. II. Lithium depletion in dwarf components. $A \mathscr{E} A$, 326, 780-792.

Barrado y Navascués, D. \& Stauffer, J. R., 1996. Lithium abundance in binaries of the Hyades open cluster. A\&A, 310, 879-892.

Bergeron, P., Leggett, S. K. \& Ruiz, M. T., 2001. Photometric and Spectroscopic Analysis of Cool White Dwarfs with Trigonometric Parallax Measurements. ApJS, 133, 413-449.

Bergeron, P., Liebert, J. \& Fulbright, M. S., 1995. Masses of DA white dwarfs with gravitational redshift determinations. ApJ, 444, 810. 
Bergeron, P., Wesemael, F. \& Fontaine, G., 1992. On the influence of the convective efficiency on the determination of the atmospheric parameters of DA white dwarfs. ApJ, 387, 288.

Bessell, M. S., 1979. UBVRI photometry. II - The Cousins VRI system, its temperature and absolute flux calibration, and relevance for two-dimensional photometry. $P A S P$, 91, 589-607.

Bessell, M. S. \& Brett, J. M., 1988. JHKLM photometry - Standard systems, passbands, and intrinsic colors. PASP, 100, 1134-1151.

BöHm-Vitense, E., 1958. Über die Wasserstoffkonvektionszone in Sternen verschiedener Effektivtemperaturen und Leuchtkräfte. Mit 5 Textabbildungen. Zeitschrift fur Astrophysik, 46, 108-+.

Catalán, S., Isern, J., García-Berro, E., Ribas, I., Allende Prieto, C. \& Bonanos, A. Z., 2008a. The initial-final mass relationship from white dwarfs in common proper motion pairs. A\&A, 477, 213-221.

Catalán, S., Ribas, I., Isern, J. \& García-Berro, E., 2008b. WD0433+270: an old Hyades stream member or an Fe-core white dwarf? A\&A, 477, 901-906.

Chen, L., Hou, J. L. \& Wang, J. J., 2003. On the Galactic Disk Metallicity Distribution from Open Clusters. I. New Catalogs and Abundance Gradient. AJ, 125, 1397-1406.

Chereul, E., Crézé, M. \& Bienaymé, O., 1999. The distribution of nearby stars in phase space mapped by Hipparcos. Clustering and streaming among A-F type stars. $A \mathscr{E} A S, \mathbf{1 3 5}, 5-28$.

Claver, C. F., Liebert, J., Bergeron, P. \& Koester, D., 2001. The Masses of White Dwarfs in the Praesepe Open Cluster. ApJ, 563, 987-998.

Dantona, F. \& Mazzitelli, I., 1978. The progenitor masses and the luminosity function of white dwarfs. $A \mathscr{E} A, \mathbf{6 6}, 453-461$.

DE Bruijne, J. H. J., 1999. A refurbished convergent-point method for finding moving groups in the HIPPARCOS Catalogue. MNRAS, 306, 381-393.

Díaz-Pinto, A., García-Berro, E., Hernanz, M., Isern, J. \& Mochkovitch, R., 1994. The luminosity function of massive white dwarfs. $A \mathscr{E} A$, 282, 86 .

Dobbie, P. D., Napiwotzki, R., Burleigh, M. R., Barstow, M. A., Boyce, D. D., Casewell, S. L., Jameson, R. F., Hubeny, I. \& Fontaine, G., 2006. New Praesepe white dwarfs and the initial mass-final mass relation. MNRAS, 369, 383-389. 
Dobbie, P. D., Pinfield, D. J., Napiwotzki, R., Hambly, N. C., Burleigh, M. R., Barstow, M. A., Jameson, R. F. \& Hubeny, I., 2004. Praesepe and the seven white dwarfs. MNRAS, 355, L39-L43.

Domínguez, I., Chieffi, A., Limongi, M. \& Straniero, O., 1999. IntermediateMass Stars: Updated Models. ApJ, 524, 226.

Domínguez, I., Straniero, O., Tornambe, A. \& Isern, J., 1996. On the Formation of Massive C-O White Dwarfs: The Lifting Effect of Rotation. ApJ, 472, 783-+.

EgGen, O. J., 1971. Luminosities, Temperatures, and Kinematics of K-Type Dwarfs. ApJS, 22, 389-+.

Eggen, O. J., 1993a. Degenerate stars in the Hyades supercluster. AJ, 106, 642649.

EgGen, O. J., 1993b. The low mass Hyades and the evaporation of clusters. AJ, 106, 1885-1905.

Eggen, O. J. \& Greenstein, J. L., 1965. Spectra, colors, luminosities, and motions of the white dwarfs. ApJ, 141, 83-+.

Eggen, O. J. \& Greenstein, J. L., 1967. Observations of Proper-Motion Stars. III. $A p J, \mathbf{1 5 0}, 927-+$.

Eggen, O. J., Lynden-Bell, D. \& Sandage, A., 1962. Evidence from the motions of old stars that the Galaxy collapsed. ApJ, 136, 748.

Fabrika, S. N., Valyavin, G. G., Burlakova, T. E., Barsukova, E. A. \& Monin, D. N., 2000. Magnetic field measurements in white dwarfs. Magnetic field, rotation and spectrum of 40 Eri B. In Y. V. Glagolevskij \& I. I. Romanyuk, eds., Magnetic Fields of Chemically Peculiar and Related Stars, Proceedings of the International Meeting, held in Special Astrophysical Observatory of Russian AS, September 23 - 27, 1999, Eds.: Yu.V. Glagolevskij, I.I. Romanyuk, p.218-228, 218-228.

Famaey, B., Jorissen, A., Luri, X., Mayor, M., Udry, S., Dejonghe, H. \& Turon, C., 2005. Dynamical Streams in the Solar Neighbourhood. In C. Turon, K. S. O'Flaherty \& M. A. C. Perryman, eds., The Three-Dimensional Universe with Gaia, vol. 576 of ESA Special Publication, 129-+.

Famaey, B., Pont, F., Luri, X., Udry, S., Mayor, M. \& Jorissen, A., 2007. The Hyades stream: an evaporated cluster or an intrusion from the inner disk? $A \& \mathcal{G}$ A 461, 957-962. 
Farihi, J., Becklin, E. E. \& Zuckerman, B., 2005. Low-Luminosity Companions to White Dwarfs. ApJS, 161, 394-428.

Ferrario, L., Wickramasinghe, D. T., Liebert, J. \& Williams, K. A., 2005. The open-cluster initial-final mass relationship and the high-mass tail of the white dwarf distribution. MNRAS, 361, 1131.

Finley, D. S., Koester, D. \& Basri, G., 1997. The Temperature Scale and Mass Distribution of Hot DA White Dwarfs. ApJ, 488, 375--.

Fontaine, G., Brassard, P. \& Bergeron, P., 2001. The Potential of White Dwarf Cosmochronology. PASP, 113, 409.

García, E., Isern, J. \& Hernanz, M., 1997. The cooling of oxygen-neon white dwarfs. MNRAS, 289, 973-978.

García-Berro, E., Hernanz, M., Isern, J., Chabrier, G., Ségretain, L. \& Mochkovitch, R., 1996. A simple method to compute white dwarf luminosity functions. $A \mathscr{E} A S, \mathbf{1 1 7}, 13$.

García-Berro, E., Hernanz, M., Mochkovitch, R. \& Isern, J., 1988. Theoretical white-dwarf luminosity functions for two phase diagrams of the carbonoxygen dense plasma. $A \mathscr{E} A, \mathbf{1 9 3}, 141$.

García-Berro, E. \& Iben, I., 1994. On the formation and evolution of superasymptotic giant branch stars with cores processed by carbon burning. 1: SPICA to Antares. ApJ, 434, 306-318.

García-Berro, E. \& Torres, S., 1997. Monte Carlo simulations of the kinematics and luminosity function of white dwarfs. In J. Isern, M. Hernanz \& E. GarciaBerro, eds., ASSL Vol. 214: White dwarfs, 97-+.

García-Berro, E., Torres, S., Isern, J. \& Burkert, A., 1999. Monte Carlo simulations of the disc white dwarf population. MNRAS, 302, 173.

García-Berro, E., Torres, S., Isern, J. \& Burkert, A., 2004. Monte Carlo simulations of the halo white dwarf population. $A \mathscr{E} A, \mathbf{4 1 8}, 53$.

Giclas, H. L., Burnham, R. \& Thomas, N. G., 1971. Lowell proper motion survey Northern Hemisphere. The G numbered stars. 8991 stars fainter than magnitude 8 with motions $>$ 0".26/year. Flagstaff, Arizona: Lowell Observatory, 1971.

Gil-Pons, P. \& García-Berro, E., 2001. On the formation of oxygen-neon white dwarfs in close binary systems. $A \mathscr{\xi} A, \mathbf{3 7 5}, 87$. 
Girardi, L., Bertelli, G., Bressan, A., Chiosi, C., Groenewegen, M. A. T., Marigo, P., Salasnich, B. \& Weiss, A., 2002. Theoretical isochrones in several photometric systems. I. Johnson-Cousins-Glass, HST/WFPC2, HST/NICMOS, Washington, and ESO Imaging Survey filter sets. $A \& A, 391,195-212$.

Gould, A. \& Chaname, J., 2004. New Hipparcos-based Parallaxes for 424 Faint Stars. ApJS, 150, 455.

Greenstein, J. L. \& Liebert, J. W., 1990. Spectrophotometry of white dwarfs as observed at high signal-to-noise ratio. II. ApJ, 360, 662 .

Heber, U., Napiwotzki, R. \& Reid, I. N., 1997. Rotation velocities of white dwarf stars. AछA, 323, 819-826.

Holberg, J. B., Barstow, M. A. \& Burleigh, M. R., 2003. An Archive of IUE Low-Dispersion Spectra of the White Dwarf Stars. ApJS, 147, 145-166.

Holberg, J. B. \& Bergeron, P., 2006. Calibration of Synthetic Photometry Using DA White Dwarfs. AJ, 132, 1221-1233.

Holberg, J. B., Oswalt, T. D. \& Sion, E. M., 2002. A Determination of the Local Density of White Dwarf Stars. ApJ, 571, 512-518.

Houdashelt, M. L., Bell, R. A. \& Sweigart, A. V., 2000. Improved ColorTemperature Relations and Bolometric Corrections for Cool Stars. AJ, 119, 14481469.

Hubeny, I. \& LAnz, T., 1995. Non-LTE line-blanketed model atmospheres of hot stars. 1: Hybrid complete linearization/accelerated lambda iteration method. ApJ, 439, 875-904.

Hurley, J. R., Pols, O. R. \& Tout, C. A., 2000. Comprehensive analytic formulae for stellar evolution as a function of mass and metallicity. MNRAS, 315, 543 .

Isern, J., CANAL, R. \& LABAy, J., 1991. The outcome of explosive ignition of ONeMg cores - Supernovae, neutron stars, or 'iron' white dwarfs? ApJ, 372, L83-L86.

Isern, J., Catalán, S., García-Berro, E. \& Hernanz, M., 2007. Testing the Relationship between the Masses of White Dwarfs and those of their Progenitors. In A. Napiwotzki \& M. R. Burleigh, eds., Astronomical Society of the Pacific Conference Series, vol. 372 of Astronomical Society of the Pacific Conference Series, $59-+$. 
Isern, J., García-Berro, E., Hernanz, M. \& Chabrier, G., 2000. The Energetics of Crystallizing White Dwarfs Revisited Again. ApJ, 528, 397.

Isern, J., Garcia-Berro, E., Hernanz, M., Mochkovitch, R. \& Torres, S., 1998. The Halo White Dwarf Population. ApJ, 503, 239-+.

Isern, J., García-Berro, E. \& Salaris, M., 2001. What Are White Dwarfs Telling Us About the Galactic Disk and Halo? ASPC, 245, 328.

Isern, J., Mochkovitch, R., García-Berro, E. \& Hernanz, M., 1997. The Physics of Crystallizing White Dwarfs. ApJ, 485, 308.

Jeffries, R. D., James, D. J. \& Thurston, M. R., 1998. Rotation and lithium in the late-type stars of NGC 2516. MNRAS, 300, 550-560.

Johnson, H. L., Iriarte, B., Mitchell, R. I. \& Wisniewskj, W. Z., 1966. UBVRIJKL photometry of the bright stars. Communications of the Lunar and Planetary Laboratory, 4, 99-+.

Johnson, H. L., MacArthur, J. W. \& Mitchell, R. I., 1968. The spectralenergy curves of subdwarfs. ApJ, 152, 465-+.

Jordan, S., Aznar Cuadrado, R., Napiwotzki, R., Schmid, H. M. \& SolankI, S. K., 2007. The fraction of DA white dwarfs with kilo-Gauss magnetic fields. $A \mathscr{E} A, \mathbf{4 6 2}, 1097-1101$.

Kalirai, J. S., Bergeron, P., Hansen, B. M. S., Kelson, D. D., Reitzel, D. B., Rich, R. M. \& Richer, H. B., 2007a. Stellar Evolution in NGC 6791: Mass Loss on the Red Giant Branch and the Formation of Low Mass White Dwarfs. ArXiv e-prints, $\mathbf{7 0 5}$.

Kalirai, J. S., Hansen, B. M. S., Kelson, D. D., Reitzel, D. B., Rich, R. M. \& Richer, H. B., 2007b. The Initial-Final Mass Relation: First Constraints at the Low Mass End. ArXiv e-prints, 706.

Kalirai, J. S., Richer, H. B., Reitzel, D., Hansen, B. M. S.m Rich, R. M., Fahlman, G. G., Gibson, B. K. \& von Hippel, T., 2005. The Initial-Final Mass Relationship: Spectroscopy of White Dwarfs in NGC 2099 (M37). ApJ, 618, 123.

Kalirai, J. S., Ventura, P., Richer, H. B., Fahlman, G. G., Durrel, P. R., D'Antona, F., \& Marconi, G., 2001. The CFHT Open Star Cluster Survey. III. The White Dwarf Cooling Age of the Rich Open Star Cluster NGC 2099 (M37). AJ, 122, 3239 . 
Karl, C. A., Napiwotzki, R., Heber, U., Dreizler, S., Koester, D., \& REID, I. N., 2005. Rotation velocities of white dwarfs. III. DA stars with convective atmospheres. $A \mathscr{E} A, \mathbf{4 3 4}, 637$.

Kawka, A., Vennes, S., Schmidt, G. D., Wickramasinghe, D. T. \& Koch, R., 2007. Spectropolarimetric Survey of Hydrogen-rich White Dwarf Stars. ApJ, 654, 499-520.

Kepler, S. O., Kleinman, S. J., Nitta, A., Koester, D., Castanheira, B. G., Giovannini, O., Costa, A. F. M. \& Althaus, L., 2007. White dwarf mass distribution in the SDSS. MNRAS, 375, 1315-1324.

Kilic, M., Stanek, K. Z. \& Pinsonneault, M. H., 2007. The Future is Now: the Formation of Single Low Mass White Dwarfs in the Solar Neighborhood. ArXiv e-prints, 706.

Kilic, M., Winget, D. E., von Hippel, T. \& Claver, C. F., 2004. Photometric Identification of Cool White Dwarfs. AJ, 128, 1825.

Koester, D., Dreizler, S., Weidemann, V. \& Allard, N. F., 1998. Search for rotation in white dwarfs. $A \mathscr{E} A, \mathbf{3 3 8}, 612-622$.

Koester, D., Napiwotzki, R., Christlieb, N., Drechsel, H., Hagen, H.J., Heber, U., Homeier, D., Karl, C., Leibundgut, B., Moehler, S., Nelemans, G., Pauli, E.-M., Reimers, D., Renzini, A. \& Yungelson, L., 2001. High-resolution UVES/VLT spectra of white dwarfs observed for the ESO SN Ia progenitor survey (SPY). I. A $\& A, \mathbf{3 7 8}, 556-568$.

Koester, D. \& Reimers, D., 1993. Spectroscopic Identification of White Dwarfs in Galactic Clusters - Part Six - Three New White Dwarfs in NGC3532. A\& $A$, $\mathbf{2 7 5}, 479-+$.

Koester, D. \& Reimers, D., 1996. White dwarfs in open clusters. VIII. NGC 2516: a test for the mass-radius and initial-final mass relations. $A \mathscr{E} A, \mathbf{3 1 3}, 810$ 814.

Kurucz, R. L., 1979. Model atmospheres for G, F, A, B, and O stars. ApJS, 40, $1-340$.

Lanz, T. \& Hubeny, I., 1995. Non-LTE line-blanketed model atmospheres of hot stars. 2: Hot, metal-rich white dwarfs. ApJ, 439, 905-916.

LÉpine, S. \& Bongiorno, B., 2007. New Distant Companions to Known Nearby Stars. II. Faint Companions of Hipparcos Stars and the Frequency of Wide Binary Systems. AJ, 133, 889-905. 
Liebert, J., Bergeron, P. \& Holberg, J. B., 2005a. The Formation Rate and Mass and Luminosity Functions of DA White Dwarfs from the Palomar Green Survey. ApJSS, 156, 47.

Liebert, J., Dahn, C. C. \& Monet, D. G., 1988. The luminosity function of white dwarfs. ApJ, 332, 891.

Liebert, J., Young, P. A., Arnett, D., Holberg, J. B. \& Williams, K. A., 2005b. The Age and Progenitor Mass of Sirius B. ApJ, 630, L69-L72.

Luyten, W. J., 1963. Proper motion survey with the forty-eight inch schmidt telescope. I-LVII. , journal $=$.

Luyten, W. J., 1979. NLTT catalogue: volume 1, +90 degrees to +30 degrees. In New Luyten Catalogue of stars with proper motions larger than two tenths of an arcsecond, 1, 0 (1979), 0-+.

Madej, J., Należyty, M. \& Althaus, L. G., 2004. Mass distribution of DA white dwarfs in the First Data Release of the Sloan Digital Sky Survey. AE\&A, 419, L5-L8.

Marigo, P., 2001. Chemical yields from low- and intermediate-mass stars: Model predictions and basic observational constraints. A\&SA, 370, 194.

Martín, E. L., Dahm, S. \& Pavlenko, Y., 2001. Revised Ages for the Alpha Persei and Pleiades Clusters. In T. von Hippel, C. Simpson \& N. Manset, eds., ASP Conf. Ser. 245: Astrophysical Ages and Times Scales, 349-+.

Masana, E., Jordi, C. \& Ribas, I., 2006. Effective temperature scale and bolometric corrections from 2MASS photometry. A\&A, 450, 735-746.

McCook, G. P. \& Sion, E. M., 1999. A Catalog of Spectroscopically Identified White Dwarfs. ApJS, 121, 1-130.

MCWilliam, A., 1990. High-resolution spectroscopic survey of 671 GK giants. I Stellar atmosphere parameters and abundances. ApJS, 74, 1075-1128.

Mestel, L., 1952. On the theory of white dwarf stars. I. The energy sources of white dwarfs. MNRAS, 112, 583.

Meynet, G., Mermilliod, J.-C. \& Maeder, A., 1993. New dating of galactic open clusters. $A \mathscr{E} A S, \mathbf{9 8}, 477-504$.

Moehler, S., Koester, D., Zoccali, M., Ferraro, F. R., Heber, U., Napiwotzki, R. \& Renzini, A., 2004. Spectral types and masses of white dwarfs in globular clusters. $A \mathscr{G} A, \mathbf{4 2 0}, 515-525$. 
NoH, H. \& Scalo, J., 1990. History of the Milky Way star formation rate from the white dwarf luminosity function. ApJ, 352, 605.

Nordström, B., Mayor, M., Andersen, J., Holmberg, J., Pont, F., Jørgensen, B. R., Olsen, E. H., Udry, S. \& Mowlavi, N., 2004. The Geneva-Copenhagen survey of the Solar neighbourhood. Ages, metallicities, and kinematic properties of $14000 \mathrm{~F}$ and G dwarfs. A\&A, 418, 989-1019.

Oke, J. B. \& Gunn, J. E., 1983. Secondary standard stars for absolute spectrophotometry. ApJ, 266, 713.

Oláh, K., Strassmeier, K. G., Kovári, Z. \& Guinan, E. F., 2001. Timeseries photometric spot modeling. IV. The multi-periodic K5Ve binary V833 Tauri. $A \& A, \mathbf{3 7 2}, 119-129$.

Olsen, E. H., 1984. Preliminary UVBY calibrations for G and K type dwarf stars. $A \& A S, \mathbf{5 7}, 443-466$.

Oswalt, T. D., 1981. A spectroscopic study of common proper motion binaries which contain degenerate components. Ph.D. thesis, AA(Ohio State Univ., Columbus.).

Oswalt, T. D., Hintzen, P. \& Luyten, W., 1988. Identifications and limited spectroscopy for Luyten common proper motion stars with probable white dwarf components. I - Pair brighter than 17th magnitude. ApJS, 66, 391.

Oswalt, T. D., Smith, J. A., Wood, M. A. \& Hintzen, P., 1996. A lower limit of 9.5 Gyr on the age of the Galactic disk from the oldest white dwarf stars. Nature, 382, 692 .

Oswalt, T. D. \& Strunk, D., 1994. A Catalog of White Dwarfs in Wide Binaries. $A A S, \mathbf{2 6}, 901$.

Panei, J. A., Althaus, L. G. \& Benvenuto, O. G., 2000a. Mass-radius relations for white dwarf stars of different internal compositions. AESA, 353, 970-977.

Panei, J. A., Althaus, L. G. \& Benvenuto, O. G., 2000b. The evolution of iron-core white dwarfs. MNRAS, 312, 531-539.

Perryman, M. A. C., Brown, A. G. A., Lebreton, Y., Gomez, A., Turon, C., de Strobel, G. C., Mermilliod, J. C., Robichon, N., Kovalevsky, J. \& CrIfo, F., 1998. The Hyades: distance, structure, dynamics, and age. $A \mathscr{E} A$, 331, 81-120.

Perryman, M. A. C., Lindegren, L., Kovalevsky, J., Hoeg, E., Bastian, U. et AL., 1997. The HIPPARCOS Catalogue. A $\& A$, 323, L49-L52. 
Pietrinferni, A., Cassisi, S., Salaris, M. \& Castelli, F., 2004. A Large Stellar Evolution Database for Population Synthesis Studies. I. Scaled Solar Models and Isochrones. ApJ, 612, 168.

Prada Moroni, P. G. \& Straniero, O., 2002. Calibration of White Dwarf Cooling Sequences: Theoretical Uncertainty. ApJ, 581, 585-597.

Press, W. H., Teukolsky, S. A., Vetterling, W. T. \& Flannery, B. P., 1992. Numerical recipes in FORTRAN. The art of scientific computing. Cambridge: University Press, — c1992, 2nd ed.

Provencal, J. L., Shipman, H. L., Hog, E. \& Thejll, P., 1998. Testing the White Dwarf Mass-Radius Relation with HIPPARCOS. ApJ, 494, 759-+.

Provencal, J. L., Shipman, H. L., Koester, D., Wesemael, F. \& Bergeron, P., 2002. Procyon B: Outside the Iron Box. ApJ, 568, 324-334.

Ramírez, I., Allende Prieto, C. \& Lambert, D. L., 2007. Oxygen abundances in nearby stars. Clues to the formation and evolution of the Galactic disk. $A \mathscr{E} A$, 465, 271-289.

Reddy, B. E., Tomkin, J., Lambert, D. L. \& Allende Prieto, C., 2003. The chemical compositions of Galactic disc F and G dwarfs. MNRAS, 340, 304-340.

ReID, I. N., 1996. White Dwarf Masses-Gravitational Redshifts Revisited. AJ, 111, $2000-+$.

Reimers, D., 1975. Circumstellar envelopes and mass loss of red giant stars, 229256. Problems in stellar atmospheres and envelopes.

Reimers, D. \& Koester, D., 1989. Spectroscopic identification of white dwarfs in galactic clusters. V - NGC 3532. A\&A, 218, 118-122.

Reiners, A. \& Schmitt, J. H. M. M., 2003. Differential rotation in rapidly rotating F-stars. $A \mathscr{G} A, \mathbf{4 1 2}, 813-819$.

Ribas, I., Jordi, C. \& Masana, E., 2007. . A $\& A$.

Richer, H. B., Fahlman, G. G., Brewer, J., Davis, S., Kalirai, J., Stetson, P. B., Hansen, B. M. S., Rich, R. M., Ibata, R. A., Gibson, B. K. \& SharA, M., 2004. Hubble Space Telescope Observations of the Main Sequence of M4. AJ, 127, 2771-2792.

Richer, H. B., Hansen, B., Limongi, M., Chieffi, A., Straniero, O. \& Fahlman, G. G., 2000. Isochrones and Luminosity Functions for Old White Dwarfs. ApJ, 529, 318. 
Salaris, M., Domínguez, I., García-Berro, E., Hernanz, M., Isern, J. \& Mochkovitch, R., 1997. The Cooling of CO White Dwarfs: Influence of the Internal Chemical Distribution. ApJ, 486, 413.

Salaris, M., García-Berro, E., Hernanz, M., Isern, J. \& Saumon, D., 2000. The Ages of Very Cool Hydrogen-rich White Dwarfs. ApJ, 544, 1036.

Salim, S. \& Gould, A., 2003. Improved Astrometry and Photometry for the Luyten Catalog. II. Faint Stars and the Revised Catalog. ApJ, 582, 1011-1031.

Salpeter, E. E., 1955. The Luminosity Function and Stellar Evolution. ApJ, 121, $161-+$.

Schaller, G., Schaerer, D., Meynet, G. \& Maeder, A., 1992. New grids of stellar models from 0.8 to 120 solar masses at $\mathrm{Z}=0.020$ and $\mathrm{Z}=0.001$. A $\mathcal{E} A S$, 96, 269-331.

SchmidT, M., 1975. The mass of the galactic halo derived from the luminosity function of high-velocity stars. ApJ, 202, 22-29.

Schmitt, J. H. M. M., Fleming, T. A. \& Giampapa, M. S., 1995. The X-Ray View of the Low-Mass Stars in the Solar Neighborhood. ApJ, 450, 392-+.

Schultz, G., Zuckerman, B. \& Becklin, E. E., 1996. Orbital Separations of White Dwarf-Red Dwarf Binaries. ApJ, 460, 402-+.

Schwarzschild, M., 1958. Structure and evolution of the stars.. Princeton, Princeton University Press, 1958.

Segretain, L., Chabrier, G., Hernanz, M., García-Berro, E., Isern, J. \& Mochkovitch, R., 1994. Cooling theory of crystallized white dwarfs. ApJ, 434, 641.

Serenelli, A. M., Althaus, L. G., Rohrmann, R. D. \& Benvenuto, O. G., 2002. Evolution and colours of helium-core white dwarf stars: the case of lowmetallicity progenitors. MNRAS, 337, 1091-1104.

Shipman, H. L. \& Provencal, J. L., 1999. Confirming a Nobel Prize winning theory with the HIPPARCOS spacecraft: Accurately determining distances and diameters of white dwarf stars. In S.-E. Solheim \& E. G. Meistas, eds., 11th European Workshop on White Dwarfs, vol. 169 of Astronomical Society of the Pacific Conference Series, 15-+.

Silvestri, N. M., Hawley, S. L. \& Oswalt, T. D., 2005. The Chromospheric Activity and Ages of M Dwarf Stars in Wide Binary Systems. AJ, 129, 2428-2450. 
Silvestri, N. M., Oswalt, T. D., Wood, M. A., Smith, J. A., Reid, I. N. \& Sion, E. M., 2001. White Dwarfs in Common Proper Motion Binary Systems: Mass Distribution and Kinematics. AJ, 121, 503.

Silvotti, R., Voss, B., Bruni, I., Koester, D., Reimers, D., Napiwotzki, R. \& Homeier, D., 2005. Two new ZZ Ceti pulsators from the HS and HE surveys. A\&A, 443, 195-199.

Steenbook, W., 1983. Barium, magnesium and iron in seven chemically unevolved stars and the nuclear evolution of the galactic disk. A\&A, 126, 325-334.

Straniero, O., 1988. A tabulation of thermodynamical properties of fully ionized matter in stellar interiors. $A \mathscr{E} A S, \mathbf{7 6}, 157$.

TAYlOR, B. J., 2003. Statistical cataloging of archival data for luminosity class IV-V stars. II. The epoch $2001[\mathrm{Fe} / \mathrm{H}]$ catalog. A $\mathscr{E} A, \mathbf{3 9 8}, 731-738$.

Tokovinin, A., Thomas, S., Sterzik, M. \& Udry, S., 2006. Tertiary companions to close spectroscopic binaries. A\&A, 450, 681-693.

Twarog, B. A., Ashman, K. M. \& Anthony-Twarog, B. J., 1997. Some Revised Observational Constraints on the Formation and Evolution of the Galactic Disk. $A J$, 114, 2556-+.

Vassiliadis, E. \& Wood, P. R., 1993. Evolution of low- and intermediate-mass stars to the end of the assymptotic giant branch with mass loss. ApJ, 413, 641657.

VON Hippel, T., 2005. From young and hot to old and cold: comparing white dwarf cooling theory to main-sequence stellar evolution in open clusters. ApJ, 622, $565-571$.

WeGneR, G., 1973. A spectroscopic survey of southern hemisphere white dwarfs-III Binaries containing a white dwarf. MNRAS, 165, 271-294.

Wegner, G. \& Reid, I. N., 1991. Gravitational redshift determinations for white dwarfs in common proper-motion binaries. ApJ, 375, 674 .

Weidemann, V., 1977. Mass loss towards the white dwarf stage. A\&A, 59, 411-418.

Weidemann, V., 2000. Revision of the initial-to-final mass relation. $A \mathscr{G} A, \mathbf{3 6 3}$, $647-456$.

Wickramasinghe, D. T. \& Ferrario, L., 2005. The origin of the magnetic fields in white dwarfs. MNRAS, 356, 1576-1582. 
Williams, K. A., 2007. A New Look at the Empirical Initial-Final Mass Relation. In A. Napiwotzki \& M. R. Burleigh, eds., Astronomical Society of the Pacific Conference Series, vol. 372 of Astronomical Society of the Pacific Conference Series, $85-+$.

Williams, K. A., Bolte, M. \& Koester, D., 2004. An Empirical Initial-Final Mass Relation from Hot, Massive White Dwarfs in NGC 2168 (M35). ApJ, 615, 49.

Winget, D. E., Hansen, C. J., Liebert, J., van Horn, H. M., Fontaine, G., Nather, R. E., Kepler, S. O. \& Lamb, D. Q., 1987. An independent method for determining the age of the universe. ApJ, 315, 77.

Wood, M. A., 1992. Constraints on the age and evolution of the Galaxy from the white dwarf luminosity function. ApJ, 386, 539.

Wood, M. A., 1995. Theoretical White Dwarf Luminosity Functions: DA Models. In D. Koester \& K. Werner, eds., LNP Vol. 443: White Dwarfs, 41-+.

Zuckerman, B., Koester, D., Reid, I. N. \& Hünsch, M., 2003. Metal Lines in DA White Dwarfs. ApJ, 596, 477-495. 


\section{Contents}

1 Introducció 1

1.1 Evolució d'estrelles de massa baixa i intermèdia . . . . . . . . . . . 2

1.2 Estrelles centrals de nebuloses planetàries i nanes blanques . . . . . 3

1.3 Problemes pendents . . . . . . . . . . . . . . 4

1.4 Com es pot mesurar la relació massa inicial- massa final? . . . . . . 8

1.4.1 Cúmuls oberts i binàries no-interactives . . . . . . . . . 8

1.4.2 La funció de lluminositat i la distribució de masses de les nanes blanques....................... 9

1.4.3 Descripció dels mètodes utilitzats en aquest treball . . . . . . 9 



\section{Chapter 1}

\section{Introducció}

La relació massa inicial-massa final connecta la massa d'una nana blanca (massa final) amb la massa de la seva estrella progenitora a la seqüència principal (massa inicial). Aquesta funció és un ingredient clau per a estudiar un gran nombre de fenòmens de l'Astrofísica moderna. De fet, és requerida per a determinar les edats dels cúmuls globulars i les seves distàncies, per a estudiar l'evolució química de les galàxies, per a determinar el ritme de supernoves per col-lapse nuclear, per a entendre la formació estel.lar i el processos de feedback a les galàxies, i per a estudiar les propietats de la població galàctica de nanes blanques. Tot i tractar-se d'una relació tan important, aquesta funció no està gens establerta, ni des d'un punt de vista teòric ni observacional. El primer intent de determinar empíricament aquesta relació el va dur a terme Weidemann (1977), qui també va proporcionar una recent revisió (Weidemann 2000). Weidemann (1977) va establir les bases del mètode més utilitzat per a derivar les masses inicial i final de les nanes blanques. Aquest mètode típicament consisteix en realitzar observacions espectroscòpiques de nanes blanques a cúmuls oberts i derivar les seves masses inicial i final utilitzant seqüències de refredament i traces d'evolució estel-lar. Com que aquest procediment combina càlculs teòrics i dades observacionals, la relació resultant s'anomena la relació de massa inicial- massa final semi-empírica. Weidemann (1977) també va dur a terme una comparació entre mesures de masses inicials i finals de nanes blanques a cúmuls oberts amb prediccions teòriques fent servir diferents aproximacions per a expressar els processos de pèrdua de massa. Des de llavors, s'han aconseguit moltes millores, tant des del punt de vista teòric com observacional. Degut a les millores tecnològiques i la instrumentació moderna desenvolupada en els recents anys, l'actual relació massa inicialmassa final semi-empírica conté dades de més de 50 nanes blanques. Tot i això, encara hi ha molts punts en el puzle per millorar. Per exemple, encara no està clara la dependència, si existeix, entre la relació massa inicial- massa final amb la massa i metal-licitat de l'estrella progenitora, moment angular, camp magnètic... 
Per a poder identificar els paràmetres principals que tenen influència en la relació massa inicial- massa final de les nanes blanques, és molt important entendre correctament l'evolució de les seves progenitores. En aquest capítol es dona una descripció breu de l'evolució de les estrelles de massa baixa i intermèdia i descrivim els problemes que encara estan pendents de ser resolts que dificulten la determinació d'una relació massa inicial- massa final fiable.

\subsection{Evolució d'estrelles de massa baixa i intermèdia}

Les nanes blanques són el producte de l'evolució de les estrelles de massa baixa i intermèdia, que són estrelles amb masses $M \lesssim 12 M_{\odot}$. Les estrelles de massa baixa i intermèdia passen la major part de la seva vida convertint hidrogen en heli a la seqüència principal. La duració d'aquesta fase decreix amb la massa de l'estrella. Com a resultat, les estrelles comparteixen unes característiques evolutives similars, tot i que existeixen algunes diferències depenent de la massa exacte de l'estrella. Això fa que tinguin unes propietats observacionals i estructurals diferents, així com uns camins evolutius diversos en el diagrama Hertzsprung-Russell, que representa la lluminositat de les estrelles en funció de la seva temperatura efectiva.

Quan una estrella exhaureix el seu hidrogen al nucli, la fase a la seqüència principal acaba i l'estrella comença a contraure's fins que la temperatura i la densitat són suficientment altes com per a començar a cremar hidrogen en un embolcall al voltant del nucli exhaurit d'hidrogen. Això fa que l'estrella s'expandeixi i arribi a la branca de les gegants vermelles (RGB). Arrivarà un moment en el que el nucli tindrà la temperatura de la ignició de l'heli i aquest començarà a cremar. L'estrella cremarà heli de manera estable en l'anomenada seqüència principal de l'heli. Tot i així, la duració d'aquesta fase és molt més curta que la corresponent a l'hidrogen. Quan l'heli s'esgota al nucli, l'estrella comença a cremar heli en un embolcall al voltant del nucli de carboni i l'estrella es mou en el diagrama Hertzsprung-Russell cap a la branca asimptòtica de les gegants (AGB). En aquesta fase, l'embolcall on es crema l'heli converteix l'heli en carboni i oxigen $(\mathrm{C} / \mathrm{O})$, incrementant la massa del nucli de $\mathrm{C} / \mathrm{O}$. A la mateixa vegada, el nucli es contrau lentament i, com a conseqüència, la densitat central i la temperatura augmenten. Quan l'embolcall on es crema l'heli s'apropa a l'embolcall d'hidrogen, l'embolcall d'hidrogen torna a cremar i incrementa la quantitat d'heli emmagatzemat, amb l'embolcall on es crema l'hidrogen proporcionant la major part de la lluminositat. En un cert punt, la massa acumulada d'heli arriba a una massa crítica i es torna inestable, causant una flamarada (o 'flash') de l'embolcall de l'heli. Quan aquest 'flash' té lloc, l'embolcall d'heli deixa anar de cop una gran quantitat d'energia en una escala de temps molt curta, causant l'expansió i el refredament de l'embolcall on es crema l'hidrogen. El resultat de l'expansió d'aquest embolcall és que les reaccions nuclears s'extingeixen. Com a conseqüència, la convecció fa que part del material d'heli surti a la superfície. A la mateixa veg- 
ada, com que la capa on es crema hidrogen s'apaga, la regió rica en hidrogen sota la capa convectiva es comprimeix i la seva temperatura augmenta. Això porta a que l'embolcall d'hidrogen es torni a encendre, sent un altre cop responsable de quasi tota la lluminositat de l'estrella, i tot el procés es torna a repetir. Aquest cicle és conegut com un pols tèrmic. Quan l'estrella progenitora és suficientment massiva $\left(\sim 9.0 M_{\odot}\right)$ i el nucli excedeix $\sim 1.05 M_{\odot}$, el carboni s'encén fora del centre en condicions semidegenerades abans d'arribar a la fase de polsos tèrmics a la AGB. En aquest cas, una sèrie de 'flashes' de l'embolcall de carboni tenen lloc al nucli i el resultant romanent està compost d'oxigen i neó $(\mathrm{O} / \mathrm{Ne})$, enlloc de $\mathrm{C} / \mathrm{O}$. De totes maneres, les estrelles amb nuclis de $\mathrm{O} / \mathrm{Ne}$ també experimenten polsos tèrmics quan la fase de la combustió del carboni s'ha acabat.

Es pensa que els polsos tèrmics produeixen una eficient pèrdua de massa, extraient les capes externes de l'estrella i deixant com a romanent una nana blanca. De fet, independentment de la composició del nucli, durant la fase de polsos tèrmics l'embolcall de l'estrella és força inestable i el ritme de pèrdua de massa pot ser molt alt. Desafortunadament, la nostra comprensió sobre els mecanismes que causen la pèrdua de massa és encara molt pobre. Conseqüentment, el nombre de polsos tèrmics necessaris per a treure tot l'embolcall encara no es coneix, tot i que probablement variïn d'una estrella a un altre depenent com a mínim de la metal-licitat i la massa de l'estrella. De totes maneres, és generalment acceptat que el mecanisme de pèrdua de massa està físicament lligat als 'flashes' d'hidrogen i heli, ja que durant aquesta fase la massa de l'embolcall de l'estrella decreix contínuament (mentre que el radi i la lluminositat augmenten) i això fa que el material de la superfície estigui menys lligat. Tot i que les causes de la pèrdua de massa encara no es coneixen, se sap que la seva influència durant l'evolució de les estrelles a l'AGB és molt important. El que està clar és que durant aquesta època final, el nucli mai es torna suficientment calent com per a que tinguin lloc reaccions nuclears. Enlloc d'això, les densitats al nucli es tornen molt elevades i la pressió dels electrons degenerats comença a dominar. Al contrari, el destí final de les estrelles de masses més grans $\left(M \gtrsim 12 M_{\odot}\right)$ és completament diferent. Aquestes estrelles passen per totes les fases però produeixen una supernova de tipus II.

\subsection{Estrelles centrals de nebuloses planetàries i nanes blanques}

Durant els estadis finals de pèrdua de massa, l'estrella deixa l'AGB, ja que la temperatura efectiva augmenta quasi a lluminositat constant. La fotosfera es torna molt brillant degut a l'absorció de fotons per part del material ejectat i l'estrella es veu ara com una nebulosa planetària. Llavors, els embolcalls d'hidrogen i heli ejectats s'extingeixen i la lluminositat de l'estrella disminueix ràpidament, revelant una nana blanca al centre de la nebulosa planetària. Les nanes blanques es troben a la part 
baixa de la seqüència principal corresponent a temperatures efectives altes però a lluminositats baixes, degut al seu petit radi.

Com s'ha mencionat anteriorment, segons càlculs teòrics no totes les nanes blanques tenen un nucli de carboni i oxigen, tot i que aquesta és la composició més típica. De fet, les nanes blanques amb masses més petites de $0.4 M_{\odot}$ estan fetes d'heli. L'existència d'aquesta població de baixa massa va ser descoberta per Liebert et al. (2005) com a resultat de l'anàlisi de la distribució de masses de nanes blanques de tipus DA al projecte observacional Palomar-Green. Les nanes blanques de nucli d'heli s'han interpretat com la conseqüència de l'evolució en sistemes binaris interactius, tot i que recentment Kilic et al. (2007) va proposar que també podien provenir de l'evolució d'estrelles aillades. Per una altra banda, les nanes blanques amb masses més grans que $\sim 1.05 M_{\odot}$ estan fetes d'oxigen i neó (García-Berro \& Iben 1994). Aquestes nanes blanques massives són el producte de l'evolució aïllada d'estrelles amb una massa inicial entre $8 \mathrm{i} \sim 12 M_{\odot}$. El rang de masses entre les poblacions de nanes blanques d'heli i d'oxigen-neó està cobert per les nanes blanques de carboni-oxigen. La composició exacte de carboni-oxigen al nucli depèn críticament de l'evolució durant la fase prèvia a l'AGB. En una nana blanca típica de $0.57 M_{\odot}$ (Kepler et al. 2007), l'oxigen representaria el $62 \%$ de la massa total, i la seva concentració a les capes centrals de la nana blanca seria més gran, al voltant del $85 \%$ (Salaris et al. 2000). Independentment de la proporció exacte entre carboni i oxigen, el nucli està envoltat per una capa prima d'heli pur amb una massa en el rang $10^{-2}$ $10^{-4} M_{\odot}$, i una capa encara més prima d'hidrogen (de $10^{-4}$ a $10^{-15} M_{\odot}$ ), que no és sempre present. Tot i que aquestes capes externes són molt primes, són molt opaques a la radiació i per tant, regulen l'energia que escapa de l'estrella. Conseqüentment, juguen un paper essencial en el refredament de la nana blanca.

Des d'un punt de vista observacional, només podem obtenir informació sobre les regions externes. Tenint en compte les característiques espectrals de les atmosferes de les nanes blanques, aquestes poden ser classificades com a nana blanca de tipus DA, si presenten línies d'absorció d'hidrogen, o com a no-DA, si no en presenten. Aproximadament el 85\% de les nanes blanques són de tipus DA, i de fet, aquestes són les millors reproduïdes des d'un punt de vista teòric, ja que tenen atmosferes totalment radiatives i les seves opacitats poden ser calculades amb bona precisió. Per tant, l'ajust de les línies de Balmer observades a models d'atmosferes poden proporcionar els paràmetres atmosfèrics (temperatura efectiva i gravetat superficial) d'aquests objectes amb excel-lent precisió. Per totes aquestes raons, en aquest treball només hem considerat nanes blanques d'aquest tipus espectral.

\subsection{Problemes pendents}

Després de descriure els principals processos físics que tenen lloc durant l'evolució de les estrelles de massa baixa i intermèdia podria semblar que existeix una relació 
entre la massa inicial i final de les estrelles ben determinada, però de fet, sabem que això no és així. A més a més, la determinació observacional d'aquesta relació és una feina difícil i no s'ha pogut dur a terme amb relativa precisió fins fa pocs anys. A continuació mostrarem que la relació massa inicial- massa final depèn de diferents paràmetres a part de la massa inicial de l'estrella a la seqüència principal:

- Metal-licitat: és clar que la composició química d'una estrella hauria de tenir un impacte important en la seva evolució. Com més petita sigui la metal.licitat, menys temps passarà a la seqüència principal. Valors petits de $Z$ porten a la reducció de l'opacitat sense una reducció del radi. Per tant, per a poder mantenir l'estructura, la temperatura i la lluminositat han d'augmentar amb la conseqüent reducció en la vida de l'estrella. Al mateix temps, el nucli d'heli que queda és més gran i la nana blanca resultant és més massiva que aquelles obtingudes d'estrelles amb la mateixa massa inicial però més metàl-liques. (Schwarzschild 1958). Tot i això, la dependència de la massa inicial- massa final està molt lluny de ser ben establerta. Les estrelles pateixen molts processos diferents durant la seva vida abans de tornar-se nanes blanques, processos que també depenen de la seva composició química. Per la qual cosa, té sentit esperar que la relació massa inicial- massa final també depengui de la metal-licitat. De fet, totes les relacions massa inicial- massa final teòriques calculades fins ara (e.g. Domínguez et al. 1999; Marigo 2001) prediuen que una estrella progenitora més metàl-lica produeix una nana blanca més massiva. Des d'un punt de vista observacional, aquesta dependència no s'ha confirmat clarament. En un treball recent, Kalirai et al. (2005) indiquen haver trobat alguna indicació de la dependència de la relació massa inicial- massa final amb la metal-licitat. Per altra banda, l'estudi acurat portat a terme per Williams (2007) no va confirmar aquesta dependència. De fet, aquest autor va trobar que per a una massa inicial de $3 M_{\odot}$, la metal-licitat hauria d'afectar la massa final només en $0.05 M_{\odot}$.

- Rotació: degut a la conservació del moment angular les nanes blanques haurien de rotar molt ràpidament, si una fracció significant del moment angular de la progenitora es preservés. Tot i així, per a la majoria de nanes blanques només s'han trobat límits superiors en les velocitats rotacionals en el rang 20 i $40 \mathrm{~km} \mathrm{~s}^{-1}$ (Karl et al. 2005). Per això, la conclusió general és que les nanes blanques roten lentament. Aquest resultat va ser confirmat per investigacions astereosismològiques de nanes blanques pulsants. Aquestes velocitats rotacionals petites indiquen que el moment angular dels nuclis estel-lars és transportat eficientment cap a la superfície de l'estrella per la massa perduda durant la seva evolució. En tot cas, és probable que la velocitat rotacional de l'estrella progenitora tinguès un impacte a l'estructura final de la nana blanca.

La contracció del nucli de carboni-oxigen a l'inici de la fase AGB de les estrelles 
de massa baixa i intermèdia augmenta el seu moment angular, inclús per a estrelles que inicialment roten lentament. Tot i que encara no existeix un tractament consistent de la rotació estel-lar, hi ha alguns estudis que intenten fer una aproximació d'aquest fenòmen. Domínguez et al. (1996) va utilitzar un mètode simplificat per a mostrar que si la rotació era inclosa als càlculs de models estel-lars, la massa final obtinguda era més gran. En particular, van obtenir que una estrella amb massa inicial $6.5 M_{\odot}$ i velocitat rotacional alta produïa una nana blanca amb una massa entre $1.1 \mathrm{i} 1.4 M_{\odot}$, depenent de les condicions inicials. Les condicions físiques aprop del centre de l'estrella en els models amb rotació de Domínguez et al. (1996) s'assemblen a aquells que no inclouen rotació però que corresponen a estrelles $\sim 1.5 M_{\odot}$ menys massives. Per això és raonable el sospitar que la relació massa inicial- massa final té una certa dependència amb la rotació, ja que afecta l'estructura final de les nanes blanques.

- Magnetisme: d'acord amb Wickramasinghe \& Ferrario (2005), les nanes blanques magnètiques amb camps de més de $\sim 10^{6} \mathrm{G}$ constitueixen el $10 \%$ de totes les nanes blanques i tenen una distribució de masses amb una massa mitjana de $\sim 0.93 M_{\odot}$, que és molt més gran que el valor típic $\sim 0.56 M_{\odot}$ (Bergeron et al. 1992) que correspon a les nanes blanques no-magnètiques. Tot i així, això podria estar esbiaixat per la influència que el camp magnètic pot tenir en la forma de les línies de Balmer, ja que podria fer que aquestes semblessin correspondre a una estrella amb camp gravitacional més gran. Wickramasinghe \& Ferrario (2005) van desenvolupar una sèrie de models de nanes blanques magnètiques i van arribar a la conclusió de que la relació massa inicial- massa final essencialment no es veu afectada per la presència d'un camp magnètic, i la raó de per què les nanes blanques magnètiques són més massives podria ser el fet de que les seves progenitores també eren més massives. El camp magnètic seria d'aquesta manera un romanent fòssil de la fase de la seqüència principal, la qual cosa seria consistent amb la conservació del flux magnètic. Tot i que aquesta explicació és la que té més punts favorables, també hi ha d'altres que no podem descartar totalment. Per exemple, la presència d'un camp magnètic en l'estrella progenitora podria alterar la seva evolució, afavorint el desenvolupament d'un nucli més massiu, i com a conseqüència, d'una nana blanca més massiva. Per tant, en aquest cas la relació massa inicial- massa final es veuria afectada.

L'efecte dels camps magnètics en l'evolució estel-lar poden ser avaluats mitjançant un estudi observacional de la població de nanes blanques. Les nanes blanques magnètiques poden ser identificades realitzant observacions espectropolarimètriques (Aznar Cuadrado et al. 2004) o bé avaluant l'efecte Zeeman en les línies de Balmer (Koester et al. 1998). D'aquestes dues tècniques, la que té més potencial és la de l'espectropolarimetria, ja que l'efecte Zeeman no és 
detectable en el cas de camps dèbils.

- Pèrdua de massa i mecanisme d'expulsió: com s'ha comentat anteriorment, durant l'ascens de l'estrella a l'AGB, els embolcalls són remoguts després d'una sèrie de polsos tèrmics. Tot i així, el moment en el que es produeix l'expulsió d'aquestes capes i com es produeix encara és tema de debat. Durant els polsos tèrmics, que són induïts per les inestabilitats de les capes on es cremen heli/hidrogen, la massa del nucli de carboni i oxigen augmenta com a resultat de l'acreció del carboni acabat de sintetizar pels 'flashes' d'heli. En absència de pèrdua de massa, la massa del nucli de carboni i oxigen creixeria suficientment com per a arribar a la massa de Chandrasekhar i finalment es produiria l'ignició del carboni. Tot i això, la lluminositat de les estrelles AGB observades, i els ritmes de pèrdua de massa observats en estrelles al principi de l'AGB semblen indicar que de fet és l'expulsió de les capes externes que fan que la sèrie de polsos tèrmics finalitzin. A més a més, també es pensa que la pèrdua de massa és sensible a la metal-licitat, augmentant la seva eficiència quan aquesta és baixa. Per tant, la relació massa inicial- massa final també es veuria afectada. Degut a totes aquestes incerteses, els processos de pèrdua de massa encara no són ben reproduïts teòricament. La pèrdua de massa i el mecanisme d'expulsió representen uns dels problemes pendents de solució més importants, ja que tenen un gran impacte en l'estructura final de les nanes blanques.

- Convecció: el coneixement del comportament real de la convecció és un dels punts més crucials en l'evolució estel-lar. La barreja de material al nucli en una estrella altera significativament la seva vida, ja que defineix el valor de $M_{\text {crit }}$, que és la màxima massa inicial que pot desenvolupar un nucli de carboni i oxigen degenerat, i així, capaç d'arribar a la fase AGB. La inestabilitat en contra de la convecció turbulenta normalment es tabula fent servi un criteri termodinàmic (el criteri de Schwarzschild per a un fluid químicament homogeni). Aquest criteri està basat en l'avaluació del gradient de temperatura produït pel transport radiatiu. Quan el gradient necessari és molt alt, la convecció llavors s'encarrega del transport de l'energia. També existeix la possibilitat de que el moviment convectiu no estigui localitzat només en el nucli. En aquest cas, una penetració podria fer que es barregés material en una zona radiativament estable, contribuint també al transport de l'energia. Hi ha diferents models que inclouen aquest fenòmen, que normalment és anomenat 'overshooting'. Aquestes diferents aproximacions per a explicar la convecció impliquen diferents conseqüències en el desenvolupament del nucli de l'estrella. En l'actualitat, el model més utilitzat és el de la teoria de 'mixing length'. Tot i que es tracta bàsicament d'una teoria fenomenològica, amb paràmetres arbitraris, els resultats obtinguts són bastant satisfactoris. Desafortunadament, aquesta no és una teoria universal i necessita ser calibrada. Conseqüentment, el valor exacte de 
la massa de transició entre les nanes blanques d'heli, les de carboni i oxigen i finalment les d'oxigen i neó sempre depenen dels models.

\subsection{Com es pot mesurar la relació massa inicial- massa final?}

Com ja hem mencionat, hi ha dos maneres essencials per a testejar la relació massa inicial- massa final: directament, realitzant observacions espectroscòpiques de nanes blanques amb paràmetres externs coneguts, o indirectament, estudiant la seva influència en la funció de lluminositat i la distribució de masses de les nanes blanques. A continuació les describim breument.

\subsubsection{Cúmuls oberts i binàries no-interactives}

En general, les observacions de nanes blanques aïllades no poden proporcionar informació sobre les propietats de les seves progenitores, ja que tenen unes gravetats superficials tan altes que els elements més pesats s'enfonsen en les atmosferes i no els podem detectar des d'un punt de vista observacional. Conseqüentment, qualsevol informació sobre la metal-licitat de l'estrella desapareix molt ràpidament durant la seva evolució. La metal-licitat té un paper molt important per a calcular l'edat total de la nana blanca (temps de refredament més vida de la progenitora). La majoria dels treballs fins ara s'han centrat en realitzar observacions de nanes blanques en cúmuls oberts, ja que per aquests sistemes la metal-licitat i l'edat poden ser establertes amb bona precisió (Ferrario et al. 2005; Dobbie et al. 2006). Els cúmuls oberts han fet possible obtenir una relació massa inicial- massa final semi-empírica amb dades corresponents a unes 50 nanes blanques. A l'inici d'aquest treball aquesta relació només cobria el rang de masses inicials entre $\sim 2.5 \mathrm{i} \sim 7 M_{\odot}$. Això es deu a que aquests cúmuls són relativament joves i en conseqüència, les nanes blanques són més massives, ja que les estrelles que han tingut temps d'arribar a aquest estadi també ho eren. La limitació que això representa va fer que busquessim nanes blanques en altres tipus de sistemes pels quals la metal-licitat i la seva edat total també es poguessin determinar. Aquest és el cas de les nanes blanques en binàries no-interactives o en parelles de moviment propi comú, en les que l'estrella companya és una estrella de baixa massa que es troba encara en les primeres fases d'evolució. Aquests sistemes són adequats pel nostre objectiu, ja que podem assumir que els dos membres del parell van néixer a la vegada i amb la mateixa metal-licitat (Wegner 1973; Oswalt et al. 1988). Mitjançant observacions de l'estrella companya aquests dos paràmetres (metal-licitat i edat total) poden ser ben determinats. Si a més a més, realitzem observacions espectroscòpiques de les nanes blanques i fem servir models de refredament i d'evolució estelar, les masses inicials i finals poden ser determinades. 


\subsubsection{La funció de lluminositat i la distribució de masses de les nanes blanques}

Des d'un punt de vista teòric, existeixen dos eïnes fonamentals per a estudiar les propietats de la població de nanes blanques: la funció de lluminositat i la distribució de masses. Ambdues poden ser calculades teòricament i comparades amb les dades observacionals que hi ha actualment. En aquest cas només farem servir la funció de lluminositat de les nanes blanques. La funció de lluminositat es pot definir com el nombre de nanes blanques per unitat de volum i per magnitud bolomètrica. Aquesta funció depèn de diferents paràmetres: el ritme de formació estel-lar, la funció inicial de massa, el temps de vida de les estrelles progenitores, la relació massa inicialmassa final... Per tant, podem realitzar tests indirectes de la relació massa inicialmassa final mitjançant la comparació de les prediccions teòriques de la funció de lluminositat amb les observacionals tenint en compte diferents relacions massa inicialmassa final.

\subsubsection{Descripció dels mètodes utilitzats en aquest treball}

L'objectiu principal d'aquesta tesi és el de millorar la determinació observacional de la relació massa inicial- massa final. Per tal de cumplir aquest propòsit farem servir nanes blanques en parelles de moviment propi comú. Després afegirem aquestes noves dades a les que ja hi ha a la literatura i farem una revisió de la relació massa inicial- massa final obtenint una nova relació semi-empírica. Des d'un punt de vista teòric, portarem a terme una sèrie de càlculs teòrics de la funció de lluminositat de les nanes blanques. També avaluarem la dependència que té aquesta funció amb diferents models d'evolució estel-lar, ritmes de formació estel-lar i, especialment, amb diferents relacions massa inicial- massa final, incloent la que hem derivat en aquest treball. Finalment, realitzarem una comparació d'aquests resultats amb dades observacionals. 